\title{
DETECTION AND GENOTYPING OF CRYPTOSPORIDIUM SPP. IN BIOFILMS AND CATTLE FECES FROM THE BLACK RIVER WATERSHED, JAMAICA
}

\author{
by \\ Amanda Morris \\ Bachelor of Science in Biology, Syracuse University, NY, 2012 \\ Master of Environmental Sciences, University of Guelph, ON, 2014
}

\author{
A Dissertation \\ presented to Ryerson University \\ in partial fulfillment of the \\ requirements for the degree of \\ Doctor of Philosophy \\ in the Program of \\ Environmental Applied Science and Management
}

Toronto, Ontario, Canada, 2019

CAmanda Morris, 2019 


\begin{abstract}
Author's Declaration
I hereby declare that I am the sole author of this dissertation. This is a true copy of the dissertation, including any required final revisions, as accepted by my examiners.

I authorize Ryerson University to lend this dissertation to other institutions or individuals for the purpose of scholarly research

I further authorize Ryerson University to reproduce this dissertation by photocopying or by other means, in total or in part, at the request of other institutions or individuals for the purpose of scholarly research.
\end{abstract}

I understand that my dissertation may be made electronically available to the public. 


\title{
Detection and Genotyping of Cryptosporidium spp. in Biofilms and Cattle Feces Collected from the Black River Watershed, Jamaica
}

Doctor of Philosophy, Amanda Morris, Environmental Applied Science and Management, Ryerson University, 2019

\begin{abstract}
Cryptosporidium is a protozoan parasite that causes the gastrointestinal disease cryptosporidiosis. The disease is endemic in most tropical countries including Jamaica; yet underreported from environmental sources. This is of concern because the primary source of anthropocentric cryptosporidiosis is surface water contaminated by human effluent and animal waste in runoff. This dissertation therefore, focuses on three main areas of research: 1) optimization and assessment of effective methods for detecting Cryptosporidium from environmental samples; 2) application of methods to analyze biofilm and cattle feces collected from the Black River watershed, located in the rural parish of St. Elizabeth, Jamaica; 3) molecular characterization of PCR-positive detections to identify Cryptosporidium species and genotypes, thereby provide inference to waterborne transmission, mitigation, and zoonotic potential within the region. First, foundational work of this dissertation focused on the development of in situ biofilm sampling for Cryptosporidium detection. Application was then performed in the Black River network for initial screening of oocysts from biofilms collected from 5 sites, and 119 cattle fecal specimens collected from 10 farms. Multiple techniques were employed to confirm the absence or presence of Cryptosporidium, including Immunofluorescence Assay (IFA) and Modified Acid-Fast (MAF) microscopy, Enzyme-Linked Immunosorbent Assay (ELISA), and Polymerase Chain Reaction (PCR). Results show that oocysts were widely dispersed in biofilms and cattle specimens. Highest prevalence was observed amoung dairy cattle compared to beef cattle, presumably due to confined space. Results
\end{abstract}


also highlight inconsistencies between detection methods, confirming that from environmental sources — where inhibitors are abundant and oocysts concentrations are naturally low — a single technique may be ineffective for understanding transmission dynamics. The second part of this dissertation focused on molecular characterization. Fourteen PCR-positive biofilm and cattle samples were subjected to gene sequencing and phylogeny. A low species diversity consisting of C. parvum and C. hominis were identified; the two most common species involved in anthropocentric infections. A specific $18 \mathrm{~S}$ rRNA isolate of $C$. parvum was found in both biofilm and cattle samples (with 99\% identity), indicating that a geographically distinct, clonal genotype of C. parvum potentially exists within the region. Further subtyping analysis of the gp60 locus identified one C. hominis subtype (IbA9G2), formally identified in human populations worldwide. Moreover, the IbA9G2 subtype was recently linked to calf infections in France, as well as a waterborne outbreak in Germany. In conclusion, this dissertation is the first to detect and characterize Cryptosporidium species and genotypes from surface water biofilms and cattle feces in Jamaica, providing informative data pertaining to public health and animal agriculture. Moreover, this research advocates the importance of utilizing multiple detection methods and sources for effective screening of Cryptosporidium throughout the environment. Whilst meaningful interpretations of Cryptosporidium population structures are developed, useful databases can form through analyzing a well-planned set of environmental samples. 


\section{Acknowledgement}

I would like to extend my gratitude to several people who generously contributed to work presented in this dissertation.

Special thanks to my supervisor Dr. Ong. I appreciate your guidance and immense knowledge. My Ph.D. has been a life-changing experience. Thank you for providing this wonderful opportunity. I would like to extend my sincere gratitude to committee members, Dr. McCarthy and Dr. Bostan. Thank you for your insightful feedback, encouragement, and all the time invested in our regular meetings, which not only kept me on track, but also challenged me to broaden my thinking. I cannot imagine having a more committed and resourceful committee as I did. Thank you Dr. Kroukamp, who most generously provided thought-provoking feedback. You've truly opened my eyes to capture the underlining issues raised in my dissertation. Special thanks to Thomas Pang and Evan Ronan for providing microscopic technical support.

Many thanks to Dr. Lindo who welcomed me with warm arms to join his research team at the University of West Indies in Jamaica. This research could not be possible without your coordination of campus housing, PCR training, and access to microbiology laboratories and research facilities. Also, through Dr. Lindo I was connected to his graduate student Simmoy Noble. It has been a pleasure collaborating with Simmoy on Cryptosporidium research. I appreciate our time analyzing cattle and mongoose samples. Thank you for showing me the ropes at UWI campus; I hope I did the same in exchange during your stay at Ryerson!

Last, I would like to thank the following organizations: CDC, Molecular Epidemiology Laboratory in Atlanta, GA for generously offering to subtype my positive samples; Lake Ontario Waterkeepers in Toronto for providing funding to build the biofilm sampler; and Standby Generator Services Inc. in Ajax for kindly offering welding services to construct the sampler. 


\section{Dedication}

To my family, especially my parents Faye and Delvon Morris for your love, inspiration and support. Your actions, words of empowerment and wisdom have taught me to know no boundaries, aim high and believe in myself. Special thanks to my amazing fiancé Michael Cole for your love, support and encouragement throughout this journey. 


\section{Table of Contents}

$\begin{array}{ll}\text { Author's Declaration } & \text { ii }\end{array}$

Abstract

$\begin{array}{lll}\text { Acknowledgement } & \text { v }\end{array}$

Dedication $\quad$ vi

List of Tables $\quad$ xii

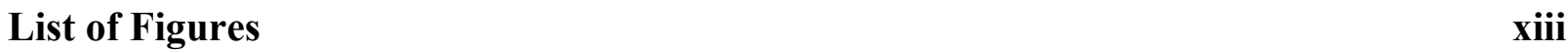

$\begin{array}{ll}\text { List of Appendices } & \text { Xv }\end{array}$

List of Abbreviations $\quad$ xvii

$\begin{array}{lc}\text { Chapter 1: Literature Review } & 1\end{array}$

1.1. Cryptosporidium and Cryptosporidiosis $\quad 1$

1.2. General Biology $\quad 2$

1.2.1. Taxonomy of the Genus Cryptosporidium $\quad 2$

1.2.2. Cryptosporidium Nomenclature and Host Specificity $\quad 4$

$\begin{array}{ll}\text { 1.2.3. The Life Cycle of Cryptosporidium } & \mathbf{8}\end{array}$

1.2.4. Cryptosporidium Transmission and Environmental Resilience 9

1.3. Historical Overview 12

1.3.1. Early Detection of Cryptosporidium in Animals $\mathbf{1 2}$

1.3.2. Increasing Economic and Public Health Interest in Cryptosporidiosis $\quad \mathbf{1 3}$

1.3.3. Significant Waterborne Outbreaks of Cryptosporidiosis $\mathbf{1 5}$

1.4. Cryptosporidium as a Global Burden 17

$\begin{array}{ll}\text { 1.5. Cryptosporidium in the Caribbean } & \mathbf{2 0}\end{array}$

1.6. Methods for Detecting and Genotyping Cryptosporidium spp. from Environmental 23 Samples 
1.6.1. Polymerase Chain Reaction (PCR) and Gene Sequencing Applications Proceeding the 1993 Cryptosporidium Outbreak in Milwaukee

1.6.2. US EPA Method 1623: Filtration/Immunomagnetic Separation (IMS)/Immunofluorescence Assay (IFA) Microscopy

1.6.3. Modified Acid-Fast (MAF) Staining: White Light Microscopy

1.6.4. Enzyme-linked Immunosorbent Assay (ELISA) Test

1.7. Specific Water Parameters that Influence the Morphology, Survival and Dispersion of Cryptosporidium Oocysts

1.8. Cryptosporidium-Biofilm Association

1.8.1. Aquatic Biofilms

1.8.2. Retention of Cryptosporidium Oocysts in Aquatic Biofilms

1.8.3. Biofilm Sampler and Water Monitoring

1.8.4. Biofilm Sampler Technique for Detection and Molecular

2.2.1. Objective \#1: Method development and evaluation of the biofilm sampler technique for Cryptosporidium oocysts entrapment and recovery efficiency from biofilm suspensions

2.2.2. Objective \#2: Development of PCR assays to optimize Cryptosporidium detection and genotyping from environmental samples using selected primers

2.2.3. Objective \#3: Detection and molecular characterization of Cryptosporidium spp. and subtypes from biofilms in the Black River and tributaries

2.2.4. Objective \#4: Detection and molecular characterization of Cryptosporidium spp. and subtypes from cattle feces in the Black River watershed 
2.2.5. Objective \# 5: Evaluation of diagnostic agreement between MAF microscopy and ELISA antigen test for the detection of Cryptosporidium from cattle feces

2.3. Summary of Experimental Workflow

2.4. General Description of Study Region

Chapter 3: Materials and Methods

52

3.1. Pilot Study: Biofilm Development and Oocyst Recovery

3.1.2. Sample Collection

3.1.3. Fluorescent Staining and Confocal Z-stack Imaging of Biofilms

3.1.4. COMSTAT2 Analyses of Biofilms Thickness and Roughness

3.1.5. Calculating IMS-recovery Efficiency of Non-viable $C$. parvum Oocysts Seeded into Biofilm Suspensions

3.2. IFA Detection of Cryptosporidium Oocysts from Biofilm Suspensions

3.2.2. Centrifugation and IMS

3.2.3. IFA Microscopy

3.3. MAF Detection of Cryptosporidium Oocysts from Cattle Feces

3.4. ELISA Detection of Cryptosporidium Antigens from Cattle Feces and Microplate Reader Interpretation

3.5. Statistical Analysis 
3.6.2. Gp60 Nested-PCR

3.6.3. Oocysts Rupture and DNA Extraction $\quad 73$

3.6.4. Spectrophotometric Analysis $\quad 74$

3.6.5. Preparation of Master Mixes $\quad 75$

3.6.6. Temperature Gradient and Thermocycler Conditions $\quad \mathbf{7 8}$

3.6.7. Gel Electrophoresis and Visualization 79

3.6.8. Purification of Positive PCR products $\quad \mathbf{8 0}$

3.7. Gene Sequencing and Phylogenetic Analysis of Cryptosporidium spp. and Subtypes 81

3.7.1. Sequencing 18S rRNA and gp60 PCR Products $\quad 81$

3.7.2. NCBI Basic Local Alignment Search Tool (BLAST) and Development $\quad 81$ of Phylogenetic Tree

3.8. ARC GIS Mapping $\quad 82$

$\begin{array}{lr}\text { Chapter 4: Results } & 85\end{array}$

4.1. Method Validation and Performance of the Biofilm Sampler Technique 85

4.1.1. Biofilm Thickness and Roughness $\quad \mathbf{8 5}$

4.1.2. Recovery Efficiency of C. parvum Oocysts Seeded into Biofilms $\quad 86$

4.2. Optimization of PCR Assays $\quad 89$

4.2.1. Spectrophotometric Analysis of Primers and Purified DNA 89

4.2.2. Temperature Gradients and Electrophoresis $\quad 91$

4.3. Detection of Cryptosporidium from Biofilms 93

4.3.1. IFA Detection $\quad 93$

4.3.2. Nested-PCR Detection and Correspondence with IFA 95

4.4. Detection of Cryptosporidium from Cattle Feces $\quad 97$

4.4.1. Comparative evaluation of MAF, ELISA and PCR techniques 97 
4.4.2. Age-related, Production Type and Diarrheic Symptom

4.4.3. Farm and Management

100

4.5. Molecular Characterization and Phylogenetic Analysis of Cryptosporidium spp. and

101 Subtypes

4.5.1. 18S rRNA Sequence Analysis and Identified Species/Genotypes

4.5.2. Phylogenetic Analysis of $18 \mathrm{~S}$ rRNA Sequences

105

4.5.3. Gp60 Sequence Analysis and Identified Subtypes

108

Chapter 5: Discussion

111

5.1. Main Findings

111

5.1.1. Assessment of in situ biofilm roughness and thickness for

111 Cryptosporidium detection

5.1.2. Biofilm sampling generates comparable oocysts recovery efficiencies to standardized filtration

113

5.1.3. Concentrated and pure DNA extracted from oocysts derived from

115 biofilm samples

5.1.4. Biofilm sampling as an alternative to conventional filtration

116

5.1.5. High prevalence of Cryptosporidium infections in dairy cattle with

118 potential association to rearing system

5.1.6. C. parvum and C. hominis isolates identified in biofilm and cattle

121 samples

5.2. Limitations and Future Work

124

5.3. Summary of Research Contributions and Outlook

127

Appendices

131

References

160 


\section{List of Tables}

Table 1.1. ICZN Classified Cryptosporidium Spp. and Hosts $\quad 5$

Table 3.1. $\quad$ Summary of Proposed Kappa Classification Scheme 70

Table 3.2. $\quad$ Primers for $18 \mathrm{~S}$ rRNA Conventional and Nested-PCR 71

$\begin{array}{lll}\text { Table 3.3. } & \text { Primers for gp60 Nested-PCR }\end{array}$

Table 3.4. $\quad$ Preparation of Master Mix with FastStart ${ }^{\mathrm{TM}}$ Taq DNA Polymerase, $\quad 76$ dNTPack for Conventional and Nested 18S rRNA PCR

Table 3.5. Preparation of Master Mix with AccuStart ${ }^{\mathrm{TM}}$ II PCR ToughMix for Conventional and Nested 18S rRNA PCR

Table 3.6. $\quad$ Preparation of Master Mix with GoTaq ${ }^{\circledR}$ DNA polymerase for gp60 Nested-PCR

Table 4.1. COMSTAT2 Analyses of Biofilm Thickness and Roughness

Table 4.2. $\quad$ IMS Recovery of $C$. parvum Oocysts from Biofilm Suspensions

Table 4.3. Concentration and Purity of Primers

Table 4.4. Concentration and Purity of Selected DNA

Table 4.5. IFA Detection of Cryptosporidium Oocysts from Biofilms (Sites A-E)

Table 4.6. Comparison Between IFA and Nested-PCR Detection of

Table 4.7. Cryptosporidium-positive Detections by MAF, ELISA and PCR in Relation to Age, Production Type and Diarrheic Symptom

Table 4.8. $\quad$ Identified Cryptosporidium sp. from Biofilm Samples by Partial Sequencing of the $18 \mathrm{~S}$ rRNA Gene

Table 4.9. Intensity Rankings of Electrophoresis Bands, Comparing Conventional and Nested-PCR-positive Detections from Cattle

Table 4.10. Identified Cryptosporidium spp. and Genotypes from Cattle by Partial 


\section{List of Figures}

Figure 1.1. Classification of Cryptosporidium in the Apicomplexa phylum 3

$\begin{array}{lll}\text { Figure 1.2. } & \text { The life cycle of Cryptosporidium } & 9\end{array}$

Figure 1.3. Environmental transmission of Cryptosporidium based on global reports 11 and research

Figure 1.4. Global sporadic outbreaks of cryptosporidiosis between 1998 and $2008 \quad 18$

Figure 1.5. Cryptosporidium oocysts under IFA, DAPI and DIC microscopy $\quad 28$

Figure 1.6. MAF stained, positive control Cryptosporidium oocysts from cattle feces $\quad 30$ captured under white light microscopy

Figure 1.7. The entrapment of $C$. parvum oocysts (red) in a $P$. aeruginosa biofilm 38 (green)

Figure 1.8. Previous biofilm samplers used for surface water monitoring $\quad 40$

Figure 2.1. $\quad$ Summary of experimental workflow 4

Figure 2.2. Fourteen parishes and twenty-six watersheds of Jamaica $\quad 5$

Figure 2.3. $\quad$ Upper and Lower Morass of the Black River watershed 51

Figure 3.1. $\quad$ Photographs and Diagram of the biofilm sampler apparatus with 53 removable slides made out of polycarbonate material

Figure 3.2. REF coverslip mounted onto a well slide to facilitate quantification of Cryptosporidium oocysts

Figure 3.3. Biofilm sampler Sites A-E in the Black River Network, St. Elizabeth, Jamaica

Figure 3.4. $\quad \mathrm{MPC}^{\mathrm{TM}}$ magnets used for oocysts purification $\quad 60$

Figure 3.5. Method 1623 examination pattern of well slide containing IFA-stained $\quad 62$ Cryptosporidium Oocysts

Figure 3.6. Cattle farms F1-F10 and distribution of fecal specimens collected in the $\quad 64$ Black River watershed, St. Elizbeth, Jamaica

Figure 3.7. PBS-ether sedimentation of cattle fecal specimen $\quad \mathbf{6 5}$ 
Figure 3.8. Map used for outlining terrestrial boundary of the Black River watershed in ARCScene10 and ARCMap10

Figure 4.1. Thin layer of biofilm formation on polycarbonate slides after 3-weeks submerged under water in biofilm sampler

Figure 4.2. Quantification of C. parvum oocysts from biofilm using IMS-IFA and REF coverslips displayed in 2D and 3D.

Figure 4.3. Relationship between turbidity and recovery efficiency Cryptosporidium oocysts seeded into environmental biofilm suspensions

Figure 4.4. NanoDrop Data Viewer- absorbance spectrum of Cryptosporidium DNA from cattle and biofilm sample

Figure 4.5. Electrophoresis images from temperature gradients using selected CPBDIAG (a), KLJ (b), and XIAO1 and XIAO2

Figure 4.6. Cryptosporidium oocysts detection from biofilms (a, b) and positive control (c) by IMS-IFA

Figure 4.7. Primary (a) and secondary (b)18S rRNA nested-PCR electrophoresis images of Cryptosporidium detection from biofilm samples, using KLJ (outer) and CPB-DIAG (inner) primers

Figure 4.8. Cryptosporidium oocysts detection from cattle sample 56DaCw (a) and positive control (b) by PBS-ether sedimentation and MAF staining

Figure 4.9. Cryptosporidium detection by three different techniques in dairy and beef cattle

Figure 4.10. Cryptosporidium detection by three different techniques in relation to farm

Figure 4.11. Phylogenetic relationships of Cryptosporidium isolates from cattle inferred by Neighbor-Joining analysis of $18 \mathrm{~S}$ rDNA sequences in MEGA7

Figure 4.12. Clustal $X$ aligned nucleotide sequences obtained from PCR-positive biofilm and cattle samples, reference species: C. parvum (MF074701.1) and $C$. hominis KX342865.1)

Figure 4.13. Percent Identity Matrix created by Clustal2.1, showing relationship between identified sequences with reference species

Figure 4.14. Secondary Nested-PCR electrophoresis image of Cryptosporidium detection from cattle samples at the gp60 locus and corresponding subtype identification 


\section{List of Appendices}

Appendix A Suppliers and Catalogue Numbers of Reagents and Kits 131

$\begin{array}{lll}\text { Table A.I. } & \text { Reagents and Kits for IMS-IFA Staining } & 131\end{array}$

Table A.II. $\quad$ Reagents and Kit for PBS-Ether Sedimentation and MAF Staining $\quad 131$

$\begin{array}{lll}\text { Table A.III. } & \text { Reagents for Biofilm Staining } & 131\end{array}$

Table A.IV. $\quad$ Reagents and Kits for ELISA 132

Table A.V. $\quad$ Reagents and Kits for DNA Extraction 132

Table A.VI. Reagents and Kits for Conventional/Nested-PCR and Electrophoresis 132

Table A.VII $\quad$ Reagents and Kit for PCR Purification 132

Table A.VIII. Reagents and Kits for Gene Sequencing 133

$\begin{array}{lll}\text { Appendix B } & \text { Maps of the Black River Watershed }\end{array}$

Figure B.I. $\quad$ Topography map of the Black River watershed and sampling locations 134 created in ARCMap10

Figure B.II. $\quad 3 D$ Map of the Black River watershed from different point of views and 135 sampling locations created in ARCScene10

$\begin{array}{lll}\text { Appendix C } & \text { Complete Data Sets } & 136\end{array}$

$\begin{array}{lll}\text { Table C.I. } & \text { Qualitative Records of Cattle Sample Collection }\end{array}$

Table C.II. $\quad$ MAF/ELISA/PCR Detections of Cryptosporidium in Cattle Feces 139 According to Age, Production Type and Diarrheal Symptom

Table C.III. Cattle Population Density per Hectare of Land in St. Elizabeth as of 140 October 2017

$\begin{array}{lll}\text { Table C.IV. } & \text { Farm Management Data } & 141\end{array}$

Table C.V. $\quad$ COMSTAT2 Analyses of Biofilm Segments 142

Table C.VI. Recovery of C. Parvum Oocysts from Biofilm Suspensions by IMS 143

Table C.VII. $\quad$ Spectrophotometric (NanoDrop ND1000) Readings 144 
Appendix D Electrophoresis Images of Cryptosporidium Detection from Cattle Feces and Positive Controls

Figure D.I. Electrophoresis images of Cryptosporidium detection derived from

149 conventional PCR products targeting the $435 \mathrm{bp}$ of the $18 \mathrm{~S}$ rRNA

Figure D.II. Electrophoresis images of Cryptosporidium detection derived from 150 nested-PCR products targeting the $1325 \mathrm{bp}$ (first round) and 819-835 bp (second round) of the $18 \mathrm{~S}$ rRNA.

Figure D.III. Electrophoresis images confirming DNA used for Cryptosporidium Positive Controls

Appendix E Chromatogram Readings of 18S rRNA Multiple Sequence Alignments

153

Figure E.I. Chromatogram readings of 18S rRNA sequences derived from

153

Cryptosporidium spp. identified in biofilm and cattle samples

Appendix F Cost Comparison of EPA Method 1623 and the Biofilm Sampler Technique

154

Table F.I. Approximate Equipment and Service Costs of Cryptosporidium Detection by Method 1623-Filtration/IMS/IFA

Table F.II. Approximate Equipment and Service Costs of Cryptosporidium Detection by Biofilm Sampler Technique- IMS/IFA

154

Table F.III. Approximate Equipment and Service Costs of Cryptosporidium Detection by Biofilm Sampler Technique- IMS/PCR

Table F.IV. $\quad$ Cost Comparison Analysis of MEHODS 1, 2 \& 3

Figure F.I. $\quad$ Cost-comparison analysis of EPA Method 1623 (filtration/IMS/IFA) 156 and two versions of the biofilm sampler technique (IMS/IFA \& IMS/PCR)

Appendix G Photographs of Biofilm Sampling Locations and Cattle Farms

$\begin{array}{lll}\text { Figure G.I. } & \text { Photographs of biofilm sampling sites A-E } & \mathbf{1 5 7}\end{array}$

$\begin{array}{lll}\text { Figure G.II. } & \text { Photographs of dairy and beef cattle, and rearing systems }\end{array}$

$\begin{array}{ll}\text { Appendix H } & 159\end{array}$

Figure H.I. Three-dimensional, z-stack imaging of biofilm segments $\quad 159$ 


\section{List of Abbreviations}

\begin{tabular}{|c|c|}
\hline AIDS & acquired immune deficiency syndrome \\
\hline BLAST & basic local alignment search tool \\
\hline BSA & Bovine Serum Albumin \\
\hline BS-C-1 & monkey kidney epithelial cells \\
\hline BFTE & bovine fallopian tube epithelial cells \\
\hline Caco-2 & human epithelial colorectal adenocarcinoma cells \\
\hline CC-PCR & cell culture-Polymerase Chain Reaction \\
\hline $\mathrm{CDC}$ & Centers for Disease Control and Prevention \\
\hline CFSPH & Center for Food Security \& Public Health \\
\hline DAPI & 4',6-diamidino-2-phenylindole \\
\hline DEM & Digital Elevation Model \\
\hline DIC & differential interference contrast \\
\hline DNA & deoxyribonucleic acid \\
\hline DO & dissolved oxygen \\
\hline DOC & Dissolved Organic Carbon \\
\hline ELISA & Enzyme-Linked Immunosorbent Assay \\
\hline EPA & Environmental Protection Agency \\
\hline EPS & extracellular polymeric substances \\
\hline FISH & Fluorescent in situ Hybridization \\
\hline FMS & Faculty of Medical Sciences \\
\hline GEMS & Global Enteric Multicenter Study \\
\hline
\end{tabular}




$\begin{array}{ll}\text { GIS } & \text { Geographic Information System } \\ \text { gp60 } & \text { 60-kDa glycoprotein } \\ \text { HAART } & \text { Highly Active Antiretroviral Therapy } \\ \text { HIV } & \text { human immunodeficiency virus } \\ \text { HCT-8 } & \text { human colon epithelial cells } \\ \text { hsp70 } & \text { Heat shock protein 70 } \\ \text { HWTP } & \text { Howard Avenue Water Treatment Plant } \\ \text { JPAT } & \text { Jamaican Protected Areas Trust Limited } \\ \text { ICR } & \text { Information Collection Rule } \\ \text { IOM } & \text { Institute of Medicine } \\ \text { IFA } & \text { Immunofluorescence Assay } \\ \text { IMS } & \text { Immunomagnetic Separation } \\ \text { ICZN } & \text { International Code of Zoological Nomenclature } \\ \text { KCF } & \text { Kinyoun's Carbol Fuchsin } \\ \text { MAF } & \text { modified acid-fast } \\ \text { Mal-ED } & \text { Malnutrition and the Consequences for Child Health and Development } \\ \text { MDCK } & \text { Madin-Darby Canine Kidney } \\ \text { MPC } & \text { Magnetic Particle Concentrator } \\ \text { mRNA } & \text { messenger ribosomal ribonucleic acid } \\ \text { MWW } & \text { Milwaukee Water Works } \\ \text { NCBI } & \text { National Center Biotechnology Information } \\ \text { NEPA } & \text { National Environmental and Planning Agency } \\ \text { NIH } & \text { NOM }\end{array}$




\begin{tabular}{ll} 
NTU & nephelometric turbidity units \\
PCR & Polymerase Chain Reaction \\
PWD & Philadelphia Water Department \\
RADA & Rural Agricultural Development Authority \\
REF & Relocatable-grid reference \\
RT-PCR & reverse transcription-Polymerase Chain Reaction \\
rRNA & ribosomal ribonucleic acid \\
RSD & relative standard deviation \\
S.D. & standard deviation \\
sp. & species singular \\
spp. & species pluralis \\
SSU & small-subunit \\
TCAG & The Centre for Applied Genomics \\
TSB & tryptic soy broth \\
USDA & United States Department of Agriculture \\
UHWI & University Hospital of the West Indies \\
UK & United Kingston \\
US & United States \\
UV & ultra-violet \\
UWI & University of the West Indies \\
WHO & World Health Organization \\
\hline
\end{tabular}




\section{Chapter 1: Literature Review}

\subsection{Cryptosporidium and Cryptosporidiosis}

The genus Cryptosporidium is a protozoan parasite that causes the gastrointestinal disease cryptosporidiosis in humans and many other vertebrate species. The term "pervasive" most accurately describes Cryptosporidium because the parasite is globally widespread and persists under various climate conditions. In addition, Cryptosporidium has been detected in at least 260 vertebrate species from all classes including mammals, reptiles, birds, fishes and amphibians (Fayer \& Xiao, 2008). The manifestation of cryptosporidiosis typically occurs when a suitable host ingests Cryptosporidium oocysts (spore-like eggs) from fecal-contaminated water, food or other sources. On rare occasions, Cryptosporidium spp. have been found in respiratory infections due to the inhalation of emitted aerosols (Spickler, 2013).

Upon ingestion by a suitable host, the most common symptom of cryptosporidiosis is watery diarrhea. Other symptoms include: stomach cramps, dehydration, nausea, vomiting, fever, weight loss, muscle aches and poor appetite (Centers for Disease Control and Prevention [CDC], 2016; Spickler, 2013). The duration and severity of symptoms depend largely on the age and immune status of the individual. In humans, symptoms transpire 2 to 10 days after infection, and can last between 1 and 2 weeks (CDC, 2016a). Similarly, healthy animals experience symptoms a few days after infection, and exhibit sporadic recovery between 1 and 2 weeks (Fayer \& Xiao, 2008; O'Donoghue, 1995). Also, some humans and animals carry the disease without showing signs of illness (Cacciò \& Widmer, 2013; CDC, 2016; CFSPH, 2014; O’Donoghue, 1995; Spickler, 2013).

In most cases, cryptosporidiosis is characterized as an acute, self-limiting infection. In general, 
healthy individuals infected by the disease recover without medical treatment. However, in immunocompromised individuals, cryptosporidiosis can be chronic and life threatening, especially in HIV/AIDS, cancer and transplant patients. For treatment of HIV/AIDS patients, anti-retroviral therapy is usually administrated. The procedure uses multiple drugs to improve the immune status of individuals thereby inhibits Cryptosporidium. However, therapeutic treatment is sometimes ineffective and severe symptoms often worsen or lead to death (CDC, 2016a; Fayer $\&$ Xiao, 2008). The elderly, young, malnourished and pregnant are also vulnerable to the disease (Cacciò \& Widmer, 2013; Putignani \& Menichella, 2010). To assist in recovery, fluid rehydration, electrolyte correction and antibiotics such as nitazoxanide are generally prescribed (CDC, 2016a; Fayer \& Xiao, 2008).

In animals, the success of antiprotozoal therapy is reportedly inconsistent. Available antiprotozoal medications have either suppressed oocysts shedding or resulted in poor clinical improvements. Also, natural remedies have shown to antidotally reduce the severity of symptoms in livestock; though the effectiveness of this form of treatment is supported by limited scientific research (Shahiduzzaman \& Daugschies, 2012).

\subsection{General Biology}

\subsubsection{Taxonomy of the Genus Cryptosporidium}

Cryptosporidium is a genus within the Apicomplexa phylum - a group of parasitic eukaryotes that have an apical complex, which is a subcellular structure designed for host invasion and attachment (Gubbels \& Duraisingh, 2012; Okamoto \& Keeling, 2014). Within the Apicomplexa phylum, Cryptosporidium was traditionally classified as a coccidian because of its life cycle phases including sexual reproduction by gamogony and asexual reproduction by merogony and 
sporogony (section 1.2.3.). However, ultrastructural studies and molecular evidence now demonstrate that Cryptosporidium is not a coccidian, but a gregarine. Figure 1.1. illustrates the taxonomic discrepancy of Cryptosporidium classification under the Apicomplexa phylum.

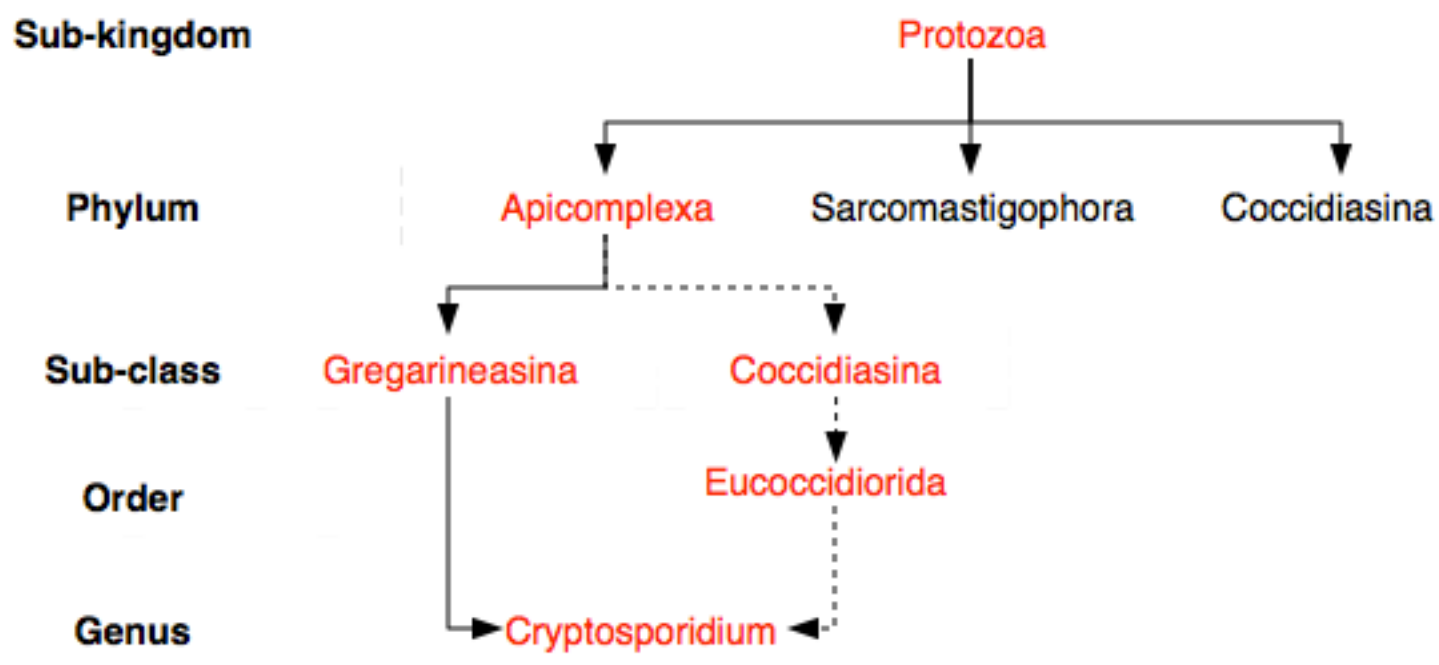

Figure 1.1.: Classification of Cryptosporidium in the Apicomplexa phylum. Includes gregarineasina and coccidiasina sub-classes. Broken arrows indicate traditional classification of Cryptosporidium. Solid arrows indicate modified classification. Taxonomic categories are shown in bold (left).

Cryptosporidium has attributes that separates it from other coccidia including its small oocysts size, distinct attachment organelle, ability to reproduce two thin-walled and thick-walled oocysts, and insensitivity to anticoccidial drugs (Cacciò \& Widmer, 2013; McDonald et al.,1990; Tenter et al., 2002; Xiao, Fayer, Ryan, \& Upton, 2004). In addition, recent genetic analyses of Cryptosporidium spp. determined that the parasite is no longer a coccidian, but a gregarine because of its ability to multiply outside hosts (Clode, Koh, \& Thompson, 2015); though this novel perception is highly debateable, thus warrants further investigation. Other key similarities 
between Cryptosporidium and gregarines include: their large extracellular gamont stages and ability to modify their cell structure for environmental adaptation (Ryan, Paparini, Monis, \& Hijjawi, 2016). Last, molecular evidence comparing the small-subunit (SSU) ribosomal ribonucleic acid (rRNA) of several gregarines and other Apicomplexan sequences determined that the phylogenetic affinity of Cryptosporidium was more closely related to gregarines than coccidia (Carreno, Matrin, \& Barta, 1999).

\subsubsection{Cryptosporidium Nomenclature and Host Specificity}

Traditional nomenclature of Cryptosporidium spp. was based on host specificity. Newly discovered species were named after their host origin such as C. bovis from cattle (Fayer, Santin, \& Xiao, 2005); C. canis from dogs (Fayer et al., 2001); C. felis from cats (Iseki, 1979); and C. serpentis from snakes etc. (Levine, 1980). Today, cross-transmission studies have revealed the heterogeneity of Cryptosporidium spp. in humans and animals. For example, C. meleagridis was traditionally linked to turkeys (Slavin,1955) and other bird species (Lindsay, Blagburn, \& Sundermann, 1989). However, since its first reported case in humans (Morgan et al., 2000), there has been an increasing number of molecular investigations finding $C$. meleagridis responsible for anthropocentric infections (Akiyoshi, 2003; Chappell, 2011; Gatei, 2008). Thus, the practice of naming species after their host origin is no longer customary. Modern research now focuses on synonymizing the names of "new" Cryptosporidium spp., in the same manner as C. parvum (Xiao et al., 2004).

Nomenclature has also been established for naming subtypes of Cryptosporidium spp., which distinguishes intra-species variation based on sequencing of the $60 \mathrm{kDa}$ glycoprotein (gp60) gene. The naming system begins with the family designation. For instance, the subtype families 
of C. hominis are Ia, Ib, Ic, Id etc.; C. parvum are IIa, IIb, IIc, IId, etc.; C. fayeri are IVa, IVb, IVc, IVd etc. Second, the nomenclature provides the dominant trinucleotide sequences and the number of repeated copies. For example, an existing subtype of $C$. hominis is IeA12G3T3, where A12 denotes 12 copies of the TCA sequence; G3 denotes 3 copies of the TCG sequence; and T3 denotes 3 copies of the TCT sequence (Xiao \& Feng, 2017). Last, a R1, R2, R3 etc. sometimes appear at the end of the naming system (e.g., C. hominis subtype IaA17R2) to distinguish the number of other repetitive sequences that may exist (Ryan, Fayer, \& Xiao, 2014).

To date, scientists have classified at least 24 species/genotypes, as well as 65 subtype families of Cryptosporidium — with new discoveries continuously authenticated (Fayer, 2010; Xiao \& Feng, 2017). Table 1.1. provides an updated list of the classified Cryptosporidium spp., along with their hosts. The data includes only formal and scientifically-named species, based on principals outlined by the International Code of Zoological Nomenclature (ICZN) (ICZN, 2016; Xiao et al., 2004).

Table 1.1.: ICZN Classified Cryptosporidium Spp. and Hosts

\begin{tabular}{|l|l|l|l|}
\hline $\begin{array}{l}\text { Species/ } \\
\text { Genotypes }\end{array}$ & $\begin{array}{l}\text { Subtype } \\
\text { Family }\end{array}$ & Hosts & References \\
\hline C. andersoni & N/A & $\begin{array}{l}\text { Bos taurus (Cattle) }{ }^{1} \text { and other } \\
\text { bovine animals; Mus musculus } \\
\text { (Mice); Ovis Aries (Sheep); } \\
\text { Camelus (Camels) }\end{array}$ & $\begin{array}{l}\text { Lindsay et al. } \\
\text { (2000) }\end{array}$ \\
\hline C. baileyi & N/A & $\begin{array}{l}\text { Gallus gallus (Chicken) }{ }^{1} \text {; Homo } \\
\text { sapiens (Humans); Coturnix } \\
\text { coturnix (Quails); Struthio camelus } \\
\text { (Ostriches); Anatidae (Ducks) }\end{array}$ & $\begin{array}{l}\text { Current, Upton and } \\
\text { Haynes (1986) }\end{array}$ \\
\hline C. bovis & N/A & $\begin{array}{l}\text { Bos taurus (Cattle) })^{1} \text { and other } \\
\text { bovine animals; Ovis Aries (Sheep) }\end{array}$ & $\begin{array}{l}\text { Fayer, Santin and } \\
\text { Xiao (2005) }\end{array}$ \\
\hline C. canis & N/A & $\begin{array}{l}\text { Canis lupus (Dogs) }{ }^{1} \text {; Homo sapiens } \\
\text { (Humans) }\end{array}$ & Fayer et al. (2001) \\
\hline
\end{tabular}




\begin{tabular}{|c|c|c|c|}
\hline $\begin{array}{l}\text { Chipmunk } \\
\text { genotype I }\end{array}$ & $\mathrm{XIVa}$ & $\begin{array}{l}\text { Tamias (Chipmunks) }{ }^{1} \text {; Sciuridae } \\
\text { (Squirrels); Mus musculus (Mice); } \\
\text { Homo sapiens (Humans) }\end{array}$ & $\begin{array}{l}\text { As cited in Xiao } \\
\text { and Feng (2017) }\end{array}$ \\
\hline C. cuniculus & $\mathrm{Va}, \mathrm{Vb}$ & $\begin{array}{l}\text { Oryctolagus cuniculus (Rabbits) } \\
\text { Homo sapiens (Humans) }\end{array}$ & $\begin{array}{l}\text { Inman and } \\
\text { Takeuchi (1979) }\end{array}$ \\
\hline C. erinacei & XIIIa & Erinaceinae (Hedgehogs) ${ }^{1}$ & Kváč et al. (2014) \\
\hline $\begin{array}{l}\text { Environmental } \\
\text { sequence }\end{array}$ & N/A & N/A & $\begin{array}{l}\text { Ruecker et al. } \\
(2011) \\
\text { (GenBank direct } \\
\text { submission) }\end{array}$ \\
\hline $\begin{array}{l}\text { Ferret } \\
\text { genotype }\end{array}$ & VIIIa & Mustela putorius furo (Ferret) & $\begin{array}{l}\text { As cited in Xiao } \\
\text { and Feng (2017) }\end{array}$ \\
\hline C. fayeri & $\begin{array}{l}\text { IVa, IVb, IVc, } \\
\text { IVd, IVe, IVf }\end{array}$ & Macropus rufus (Kangaroos) ${ }^{1}$ & $\begin{array}{l}\text { Ryan, Power and } \\
\text { Xiao (2008) }\end{array}$ \\
\hline C. felis & N/A & $\begin{array}{l}\text { Felis catis }\left(\text { Cats) }{ }^{1} \text {; Homo sapiens }\right. \\
\text { (Humans); Bos taurus (Cattle) }\end{array}$ & Iseki (1979) \\
\hline C. galli & N/A & Gallus gallus (Chicken) $^{1}$ & Pavlásek (1999) \\
\hline C. hominis & $\begin{array}{l}\text { Ia, Ib, Id, Ie, If, } \\
\text { Ig, Ih, Ii. Ij, Ik }\end{array}$ & $\begin{array}{l}\text { Homo sapiens (Humans) }{ }^{1} \text {; Bos } \\
\text { taurus (Cattle); Ovis Aries (Sheep); } \\
\text { Equidae (Horses) }\end{array}$ & $\begin{array}{l}\text { Morgan-Ryan et al. } \\
(2002)\end{array}$ \\
\hline $\begin{array}{l}\text { Horse } \\
\text { genotype }\end{array}$ & VIa, VIb, VIc & Equus caballus (Horses) $^{1}$ & $\begin{array}{l}\text { As cited in Xiao } \\
\text { and Feng (2017) }\end{array}$ \\
\hline C. meleagridis & $\begin{array}{l}\text { IIIa, IIIb, IIIc, } \\
\text { IIId, IIIe, IIIf, } \\
\text { IIIg, IIIh, IIIi, } \\
\text { IIIj }\end{array}$ & $\begin{array}{l}\text { Meleagris gallopavo (Turkey) })^{1} \text { and } \\
\text { other galliformes; Homo sapiens } \\
\text { (Humans); } \\
\text { Psittaciformes (Parrots) }\end{array}$ & Slavin (1955) \\
\hline $\begin{array}{l}\text { Mink } \\
\text { genotype }\end{array}$ & $\mathrm{Xa}$ & $\begin{array}{l}\text { Neovison vison }(\text { Minks })^{1} ; \text { Lutrinae } \\
\text { (Otters); Mustela (Ermines) }\end{array}$ & $\begin{array}{l}\text { As cited in Xiao } \\
\text { and Feng (2017) }\end{array}$ \\
\hline C. molnari & N/A & $\begin{array}{l}\text { Sparus auratus }{ }^{1} \text { and Dicentrarchus } \\
\text { labrax }^{1} \text { (Fishes) }\end{array}$ & $\begin{array}{l}\text { Alvarez-Pellitero } \\
\text { and Sitjà-Bobadilla } \\
(2002)\end{array}$ \\
\hline C. muris & N/A & $\begin{array}{l}\text { Mus musculus (Mice) }{ }^{1} \text { and other } \\
\text { rodents; Homo sapiens (Humans); } \\
\text { Bos taurus (Cattle); Capra } \\
\text { aegagrus hircus (Goat) }\end{array}$ & Tyzzer (1910) \\
\hline
\end{tabular}




\begin{tabular}{|c|c|c|c|}
\hline C. nasorum & N/A & Naso lituratus (Fishes) ${ }^{1}$ & $\begin{array}{l}\text { Hoover, Hoerr, } \\
\text { Carlton, Hinsman } \\
\text { and Ferguson } \\
(1981)\end{array}$ \\
\hline $\begin{array}{l}\text { Opossum } \\
\text { genotype }\end{array}$ & XIa & Didelphimorphia (Opossum) ${ }^{1}$ & $\begin{array}{l}\text { As cited in Xiao } \\
\text { and Feng (2017) }\end{array}$ \\
\hline C. parvum & $\begin{array}{l}\text { IIa, IIb, IIc, IId, } \\
\text { IIe, IIf, IIg, IIh, } \\
\text { IIi, IIk, IIl, IIm, } \\
\text { IIn, IIo, IIp, IIq, } \\
\text { IIr, IIs, IIt }\end{array}$ & $\begin{array}{l}\text { Mus musculus (Mice) })^{1} \text { and other } \\
\text { rodents, Homo sapiens (Humans); } \\
\text { Bos Taurus (Cattle); Ovis Aries } \\
\text { (Sheep); Sus (Pigs); } \\
\text { Capra aegagrus hircus (Goats); } \\
\text { Equidae (Horses); Cervidae (Deer) }\end{array}$ & Tyzzer (1912) \\
\hline $\begin{array}{l}\text { C. } \\
\text { saurophilum }\end{array}$ & N/A & $\begin{array}{l}\text { Squamata (Lizards) }{ }^{1} ; \text { Elaphe } \\
\text { guttata (Snake) }\end{array}$ & $\begin{array}{l}\text { Koudela and Modrý } \\
\text { (1998) }\end{array}$ \\
\hline C. scrofarum & N/A & Sus (Pigs) ${ }^{1}$ & Kváč et al. (2013) \\
\hline C. serpentis & N/A & $\begin{array}{l}\text { Elaphe guttata }\left(\text { Snake) }{ }^{1} ; \text { Squamata }\right. \\
\text { (Lizards) }\end{array}$ & Levine (1980) \\
\hline $\begin{array}{l}\text { Skunk } \\
\text { genotype }\end{array}$ & XVIa & $\begin{array}{l}\text { Mephitidae (Skunks) }{ }^{1} \text { and other } \\
\text { rodents }\end{array}$ & $\begin{array}{l}\text { As cited in Xiao } \\
\text { and Feng (2017) }\end{array}$ \\
\hline C. tyzzeri & IXa, IXb & Mus musculus (Mice) $)^{1}$ & Ren et al. (2012) \\
\hline C. ubiquitum & $\begin{array}{l}\text { XIIa, XIIb, } \\
\text { XIIc, XIId, } \\
\text { XIIe, XIIf }\end{array}$ & $\begin{array}{l}\text { Cervidae }\left(\text { Deer) }{ }^{1} \text { and other }\right. \\
\text { ruminants; rodents; Homo sapiens } \\
\text { (Humans) and other primates }\end{array}$ & $\begin{array}{l}\text { Fayer, Santín and } \\
\text { Macarisin (2010) }\end{array}$ \\
\hline C. varanii & N/A & Varanus prasinus (Monitor) $^{1}$ & $\begin{array}{l}\text { Pavlásek et al. } \\
\text { (1995) }\end{array}$ \\
\hline C. viatorum & $\mathrm{XVa}$ & Homo sapiens (Humans) ${ }^{1}$ & Elwin et al. (2012) \\
\hline C. wrairi & VIIa & Guinea pig (Cavia porcellus) $)^{1}$ & $\begin{array}{l}\text { Vetterling, Jervis, } \\
\text { Merril, and Sprinz } \\
\text { (1971) }\end{array}$ \\
\hline C. xiaoi & N/A & $\begin{array}{l}\text { Ovis Aries (Sheep) }{ }^{1} \text {; Capra } \\
\text { aegagrus hircus (Goat) }\end{array}$ & $\begin{array}{l}\text { Fayer and Santín } \\
\text { (2009) }\end{array}$ \\
\hline
\end{tabular}

Sources: Smith et al. (2007); Ghazy et al. (2015); Fayer (2010); Ren et al. (2012); Xiao and Feng (2017)

${ }^{1}$ signifies the first reported host of Cryptosporidium spp. 


\subsubsection{The Life Cycle of Cryptosporidium}

Cryptosporidium initiates its life cycle as a sporulated oocysts. When the oocysts are ingested by a suitable host, excystation occurs and the genus sheds sporozoites (immature forms of the parasite) into the gastrointestinal system. The sporozoites invade the epithelial of the gastrointestinal tract and other tissues by attaching to enterocytes, which are cells of the intestinal lining. The sporozoites then transition to a growing trophozoite stage, which undergoes asexual reproduction by merogony, producing a type I meront, as well as 6-8 merozoites. The merozoites release back into the gastrointestinal track and either infect new enterocytes or transition into a type II meront, which is accompanied by 4 merozoites. All merozoitesdeveloped from both type I meront and type II meront—infect new enterocytes forming a macrogamont (female) or a microgamont (male). The macrogamont and microgamont undergo sexual reproduction by gamogony, and thus form a zygote (Cacciò \& Widmer, 2013; Leitch \& He, 2012; Tenter et al., 2002). The zygote then asexually forms 4 internal sporozoites by sporogony, resulting in thin-walled and thick-walled oocysts.

Approximately, $20 \%$ of oocysts are thin-walled and remain within the host. These oocysts rupture and re-infect the host by releasing additional sporozoites, which repeat the life cycle. The remaining oocysts are thick-walled and excrete back into the environment through fecal matter (Leitch \& He, 2012; Ryan \& Ray, 2004). Figure 1.2. shows the life cycle of Cryptosporidium including its monoxenous development, where all stages of reproduction (asexual and sexual) are occurring in a single host. 


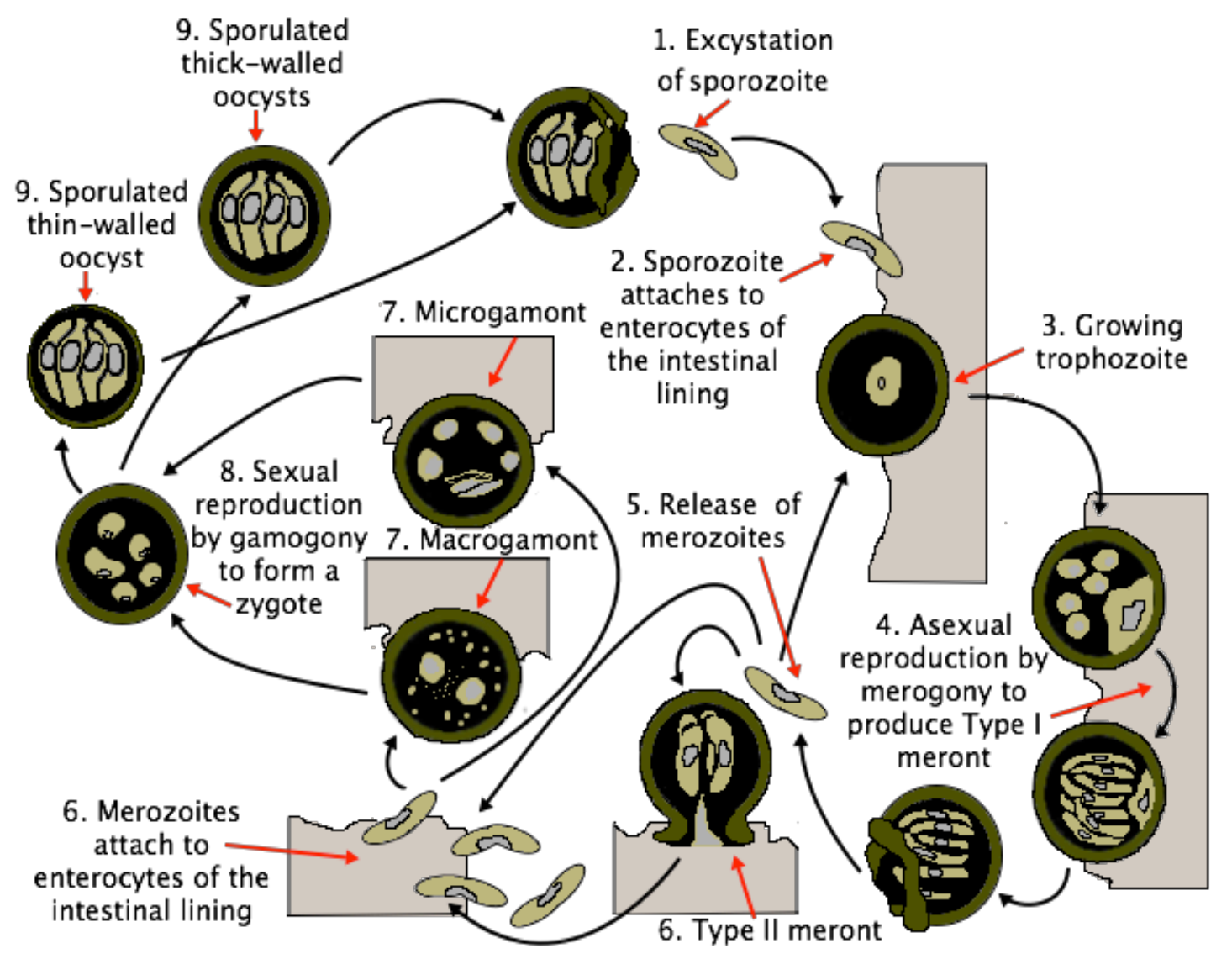

Figure 1.2.: The life cycle of Cryptosporidium. Adapted from sources: CDC (2017b); Bouzid, Hunter, Chalmers and Tyler (2013).

\subsubsection{Cryptosporidium Transmission and Environmental Resilience}

Cryptosporidium transmits via the oral-fecal route, and on rare occasions through emitted aerosols (Spickler, 2013). Figure 1.3. shows the various sources of Cryptosporidium transmission, based on global reports and research. It is evident that most sporadic outbreaks of cryptosporidiosis are traced to surface water, but infections have also been traced to groundwater and wastewater sources (Coupe et al., 2006; Corso et al., 2003; Daniels, Smith, Schmidt, Clasen, \& Jenkins, 2016; D'antonio et al., 1985; Egorov et al., 2004; Fayer \& Xiao, 2008; Gibson, 
Stadterman, \& Sykora, 1998; Hayes et al., 1989; Johnson, Pieniazek, \& Rose, 1993; Karanis et al., 2006; Putignani \& Menichella, 2010).

The spread of Cryptosporidium to surface water is largely due to animal droppings and manure applications to fertilized soils. Hofstra, Bouwman, Beusen and Medema (2013) developed a model-based inventory of global emissions and determined that oocysts transmitted from livestock to surface water is marginally higher than human emissions. The study however, did not consider transmission from wildlife populations - an area of research which warrants further investigation (Ziegler et al., 2007). For this reason, it can be expected that animal feces in soil collectively contain high volumes of oocysts compared to human feces.

Subsequent to heavy rainfall activity, water flows through and mixes with soil, producing leachate and runoff into surface water. Moreover, runoff occurs more frequently than leachate because soil provides filtration, hence most oocysts are retained in topsoil (Mawdsley, Brooks, \& Merry, 1996). Soil-dwelling oocysts are highly resistant to environmental stressors. Walker, Montemagno and Jenkins (1998) reviewed studies on the survival and transport of C. parvum and determined that oocysts can survive in dry soils for several months, under cool, shaded conditions. In addition, the deactivation of oocysts in soil occurs at 6 months, 2 months, and 72 hours when exposed to $15^{\circ} \mathrm{C}, 25^{\circ} \mathrm{C}$ and $37^{\circ} \mathrm{C}$, respectively (Fayer, Trout, \& Jenkins, 1998). Moreover, feces shield oocysts from environmental pressures by reducing permeability of small molecules (Robertson, Campbell, \& Smith, 1992). Therefore, oocysts can survive in various soils given their robust nature, and interaction with protective barriers. 
Municipal drinking water (e.g., operational failures in water treatment

- facilities) (D'antonio et al., 1985; Egorov et al., 2004; Hayes et al., 1989; Karanis et al., 2006; Putignani \& Menichella, 2010)

Drinking wells located near poorly designed latrine pits (Daniels, Smith,

$\longrightarrow$ Schmidt, Clasen, \& Jenkins, 2016) Surface and recreational water (e.g., pools, amusement parks, swimming/ bathing in lakes, rivers etc.) (Coupe et al., 2006; Daniels, Smith, Schmidt, Clasen, \& Jenkins, 2016; Putignani \& Menichella, 2010)

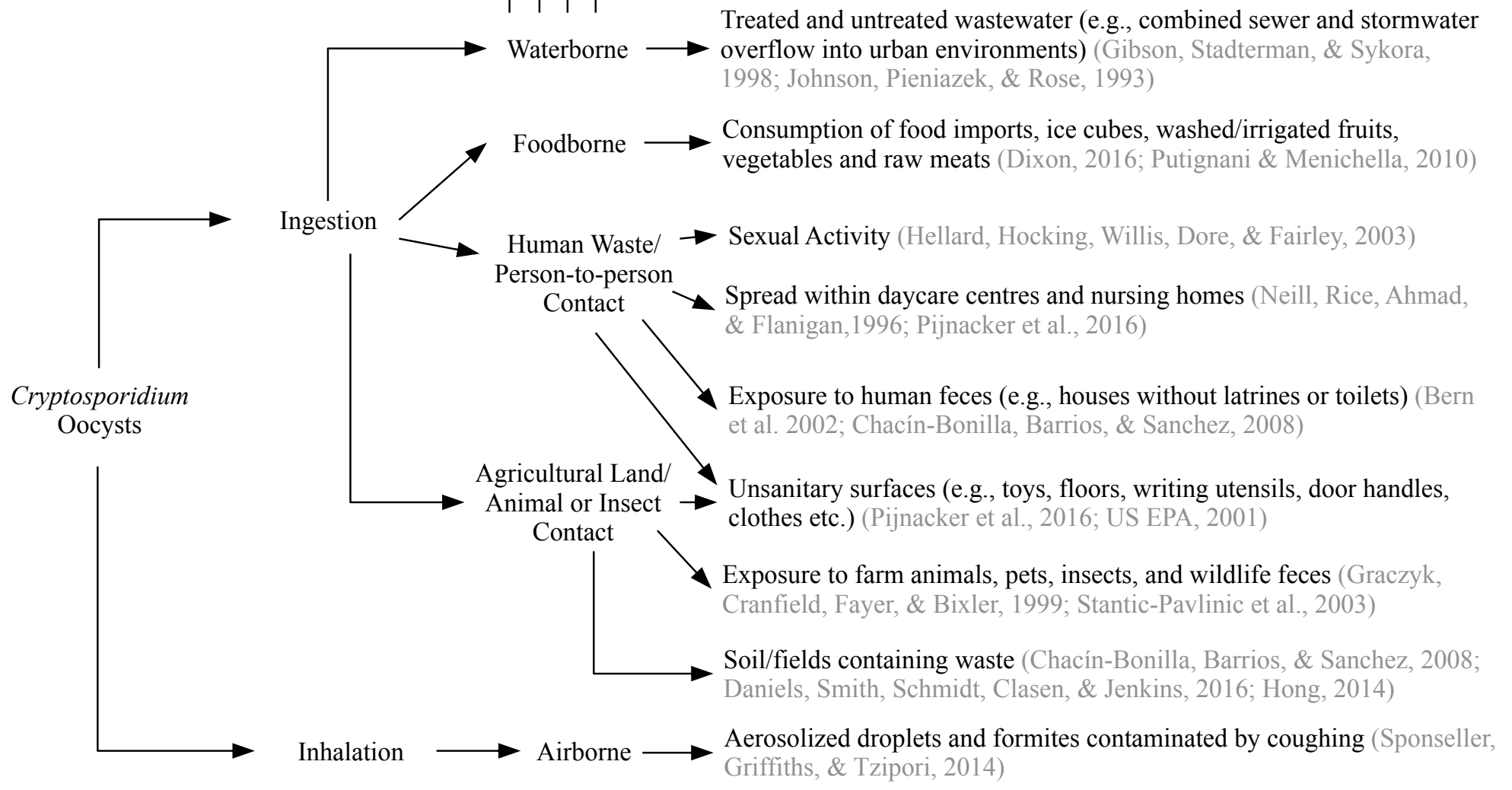

Figure 1.3.: Environmental transmission of Cryptosporidium based on global reports and research.

The four-main physiological and morphological characteristics that contribute to the environmental resilience of Cryptosporidium oocysts include:

1. Tough outer shell: resistant to extreme temperatures and most conventional disinfectants, including chlorination (at concentrations that are safe to work with).

2. Small size: permits transportation through filtration systems at water treatment plants. 
3. Infectivity: ability to cause sickness in a broad range of hosts.

4. Low-infectious dose: requires 10 oocysts in humans and 50 oocysts in cattle to contract cryptosporidiosis, whereas a single bowel movement can release $10^{7}-10^{8}$ oocysts (CDC, 2016a; DuPont et al.,1995; Moore et al., 2003).

\subsection{Historical Overview}

\subsubsection{Early Detection of Cryptosporidium in Animals}

The first publication that describes Cryptosporidium is accredited to an American physician and parasitologist by the name of Ernest Edward Tyzzer (1875-1965). Tyzzer described the parasite in the gastric glands of asymptomatic common mice using a simple light microscope. The parasite was noted to have various asexual and sexual life-cycle stages, and specialized organelles designed for the attachment to host cells. Also, spores were documented in the gastric glands of the mice, which was later described as indistinguishable or absent in the oocyst (Tyzzer, 1907). Based on these observations, Tyzzer coined the name Cryptosporidium, which translates to "hidden spore" (or Latin: "underground spore"). Shortly after, Tyzzer published two additional articles, which identified species of Cryptosporidium (also found in asymptomatic common mice). He named these species C. muris and C. parvum (Tyzzer, 1910; Tyzzer, 1912). Both publications provided further details about the physiology and life-cycle of the genus. However, Cryptosporidium research at the time lacked significance because the parasite was never known to cause sickness in humans nor animals.

By 1955, the species C. meleagridis was introduced after its discovery in turkeys. What made this discovery unique compared to prior detections was that the turkeys were experiencing symptoms of severe diarrhea. This marked the first reported case of Cryptosporidium as a 
causative agent of illness and mortality (Slavin,1955). Nonetheless, Cryptosporidium research remained inconsequential, and from 1955 to 1970 there were only a few follow-up publications, in which Cryptosporidium was detected in a wide range of animals (Anderson, Duszynski, \& Marquardt, 1968; Dubey \& Pande, 1963; Jervish, Merrillt, \& Sprinzh, 1966). Overall, Cryptosporidium research received little attention, and Tyzzer's initial three articles published in the early 1900s remained at the forefront of research, having defined most of what was known about the genus for the subsequent 43 years.

\subsubsection{Increasing Economic and Public Health Interest in Cryptosporidiosis}

During the 1970s and 1980s, Cryptosporidium was gaining economic significance. The parasite was frequently reported in symptomatic livestock including cattle, lamb, chicken and goats (Angus, Tzipori, \& Gray, 1982; Barker \& Carbonell, 1974; Nagy, Bozso, Pal, Nagy, \& Sahibi, 1984; Snyder, Current, \& Russek-Cohen, 1988; Tzipori, Campbell, Sherwood, Snodgrass, \& Whitelaw, 1980; Widmer, 1998). Amoung this group, Cryptosporidium detection in cattle was most frequent. For example, $C$. parvum was detected in the small intestine of an 8-month-old calf that died after experiencing symptoms of dehydration and watery feces (Panciera, Thomassen, \& Garner, 1971). Shortly after, many articles were published on the morbidity and mortality of dairy and beef calves due to cryptosporidiosis (Barker \& Carbonell, 1974; Meuten, Van Kruiningen, \& Lein, 1974; Morin, Lariviere, \& Lather, 1976). Young calves were found most susceptible to the disease because unlike adult cattle, calves lack mature T cells (or T lymphocytes), which are white blood cells that actively participate in immune responses. Thus, Cryptosporidium was officially ruled as a probable factor of diarrhea in newborn calves (Pohlenz, Moon, Cheville, \& Bemrick, 1978). This raised economic concerns in the cattle 
industry regarding financial losses associated with cattle weight-loss, stunted-growth and to the lesser extent mortality. Cattle farmers were also worried about increasing veterinary and labour costs, including medicine and sanitary measures used to prevent the disease from spreading to other livestock and even pets (De Graaf, Vanopdenbosch, Ortega-Mora, Abbassi, \& Peeters, 1999; Fayer \& Ungar, 1986; House, 1978).

During 1970s and 1980s, Cryptosporidium also gained public health significance due to the increasing number of zoonotic incidences. For example, in 1976 two articles were published on the first human cases of cryptosporidiosis in an immunocompromised man and toddler (Meisel, Perera, Meligro, \& Rubin, 1976; Nime, Burek, Page, Holscher, \& Yardley, 1976). Both individuals lived on a cattle farm and had a dog in the house. Shortly after, many cases followed where human cryptosporidiosis was suspected to have been contracted through direct contact with animal feces (Current, 1985; Pitlik et al., 1983). There were also reports of Cryptosporidium spreading through person-to-person contact (especially in daycare centres), and through ingestion of fecal-contaminated food and water sources (CDC, 1984; Fayer \& Ungar, 1986). By the 1980s, Cryptosporidium reached its peak in public health interest. During this era, several clinical studies revealed that the parasite was positively correlated with death in HIV and AIDS patients (Navin \& Juranek, 1984; Pitlik et al., 1983; Tzipori \& Widmer, 2008). This confirmed that Cryptosporidium was an opportunistic parasite, causing severe illness in immunocompromised individuals.

Although the heightened attention during the 1980s produced a plethora of publications, many reports mistaken Cryptosporidium for other pathogens. For example, sporocysts of Sarcocystis spp. were falsely perceived as Cryptosporidium oocysts because they are from the same Apicomplexa phylum, thus appear similar under the microscope (Levine, 1980). Nonetheless, 
ultrastructural studies re-confirmed that Cryptosporidium has a distinct attachment organelle, which is an integral component of its classification (Xiao et al., 2004) (section 1.2.1.). Hence, from the 1990s to early 2000s, research pertaining to Cryptosporidium nomenclature and host specificity was further clarified, as microscopy and molecular techniques advanced (Cacciò \& Widmer, 2013; Clode et al., 2015). Today, researchers continue to use these technologies to study the morphological characteristics of Cryptosporidium spp., as well as to enhance the specificity and sensitivity of oocysts detection from environmental sources.

\subsubsection{Significant Waterborne Outbreaks of Cryptosporidiosis}

In July 1984, the first reported outbreak of waterborne cryptosporidiosis occurred in Braun Station, Texas. An estimated 2000 people-34\% of the population-contracted cryptosporidiosis from a sewage-contaminated well. Based on these findings, D'antonio et al. (1985) recommended that Cryptosporidium should be added to the list of waterborne pathogens capable of causing outbreaks of gastroenteritis. Shortly after, in February 1987, another large-scale outbreak occurred in Western Georgia, where an estimated 13,000 residents - 39\% of the populationcontracted cryptosporidiosis. The outbreak resulted from contaminated surface water, flowing through a water treatment facility during operational irregularities (Hayes et al., 1989). In both cases, drinking-water standards adhered to the World Health Organization (WHO)'s Guidelines for Drinking Water Quality, which focused on using E. coli as an indicator pathogen for monitoring Cryptosporidium spp. (Chalmers, 2012). Thus, post investigations determined that water treatment standards and the use of surrogate pathogens were insufficient in preventing Cryptosporidium from contaminating drinking water. 
Similar conclusions were drawn following the April 1988 outbreak in Ayrshire, Scotland, which marked the first waterborne outbreak of cryptosporidiosis reported in the UK. Initial investigations diagnosed cryptosporidiosis in 27 people, and a follow up investigation determined that several hundred at the time experienced symptoms of diarrhea. The cause of the outbreak originated from the absence of fecal bacteria indicators, and operational failures during a time of heavy rainfall activity, which allowed animal slurry to contaminate the drinking water source (Smith et al., 1989). That same year leading into 1989, over 500 residents from Wiltshire and Oxfordshire, England, contracted cryptosporidiosis from treated water (Richardson et al., 1991). Evidently, these patterns continued into the early 1990s, when two large-scale outbreaks occurred in Oregon (Leland, McAnulty, Keene, \& Stevens, 1993) and North-west England (Bridgman et al., 1995). Both outbreaks resulted from inadequate filtration and disinfectants (e.g., chlorination), which failed to prevent Cryptosporidium oocysts from bypassing treatment. Despite these systematic failures, it wasn't until the massive outbreak in Milwaukee, that prompted research and operational changes worldwide.

In the spring of 1993, the largest outbreak of Cryptosporidium in history occurred in the greater area of Milwaukee, Wisconsin. Based on telephone surveys and hospital records, 69 residents died and approximately 403,000 residents $-26 \%$ of the 1.6 million population-experienced symptoms of the infection (Corso et al., 2003). At the time of the outbreak, the Milwaukee Water Works (MWW) ensured the region with potable water from Lake Michigan (Institute of medicine $[\mathrm{IOM}], 2009)$. Following an investigation, the Milwaukee outbreak was attributed to inadequate water treatment due to abnormal increases in turbidity between the months of March and April 1993. During this time, Milwaukee was experiencing greater-than-normal winds, precipitation, and snow melt (Eisenberg, Seto, Colford, Olivieri, \& Spear, 1998). All of these factors contributed to the excessive runoff of water and debris containing Cryptosporidium 
oocysts into Lake Michigan and subsequently to the Howard Avenue Water Treatment Plant (HWTP), which served one third of the population (Fox \& Lytle, 1996; Gradus, 2014). The magnitude of the outbreak posed significant threat to the US water supply. The economic costs associated with medical care and productivity losses totaled $\$ 96.2$ million (IOM, 2009). This emphasized on the need to establish better monitoring programs for Cryptosporidium. Thus, between 1993 and 2016, MWW invested a total of \$459 million in water-related technologies and projects to ensure high-quality water and monitoring services (Water Research Foundation, 2016). Today, MWW is a champion for the development of an effective multiple-barrier strategy, which is currently modeled worldwide.

\subsection{Cryptosporidium as a Global Burden}

Cryptosporidium is globally widespread in developing and industrialized nations (Figure 1.4.). The greatest burden however, occurs in developing countries (CDC, 2017a), where most reported cases are associated with $C$. parvum and $C$. hominis species (Putignani, \& Menichella, 2010), and children who suffer from famine and symptoms of moderate-to-severe diarrhea

(Guerrant, 1997; Kotloff et al., 2012). In general, persistent diarrhea is the leading cause of death in children under the age of 5 in developing countries, accounting for $30-50 \%$ of childhood mortalities (Ochoa, Salazar-Lindo, \& Cleary, 2004). Because Cryptosporidium spp. deplete the lining of the gastrointestinal track, the diarrheic disease is considered a contributing factor of environmental enteropathy - a condition derived from continuous exposure to fecal pathogens, which reduces the surface area of one's intestines, resulting in the inability to retain nutrients (Brown, Khatun, \& Ahmed, 1981). Consequently, studies have found correlations between cryptosporidiosis and stunted growth in children from Uganda (Tumwine et al., 2003), Peru 
(Checkley et al., 1998), Haiti (Kirkpatrick et al., 2006), and Bangladesh (Korpe et al., 2016).

Other correlations include: cognitive impairment, gut microbiome perturbation, malnutrition, reduced vaccination efficacy and susceptibility to other diseases (Agnew et al., 1998; Alcantara et al., 2003; Guerrant, 1997; Guerrant et al., 1998; Lang \& MAL-ED Network Investigators, 2015; Shoultz, de Hostos, \& Choy, 2016).

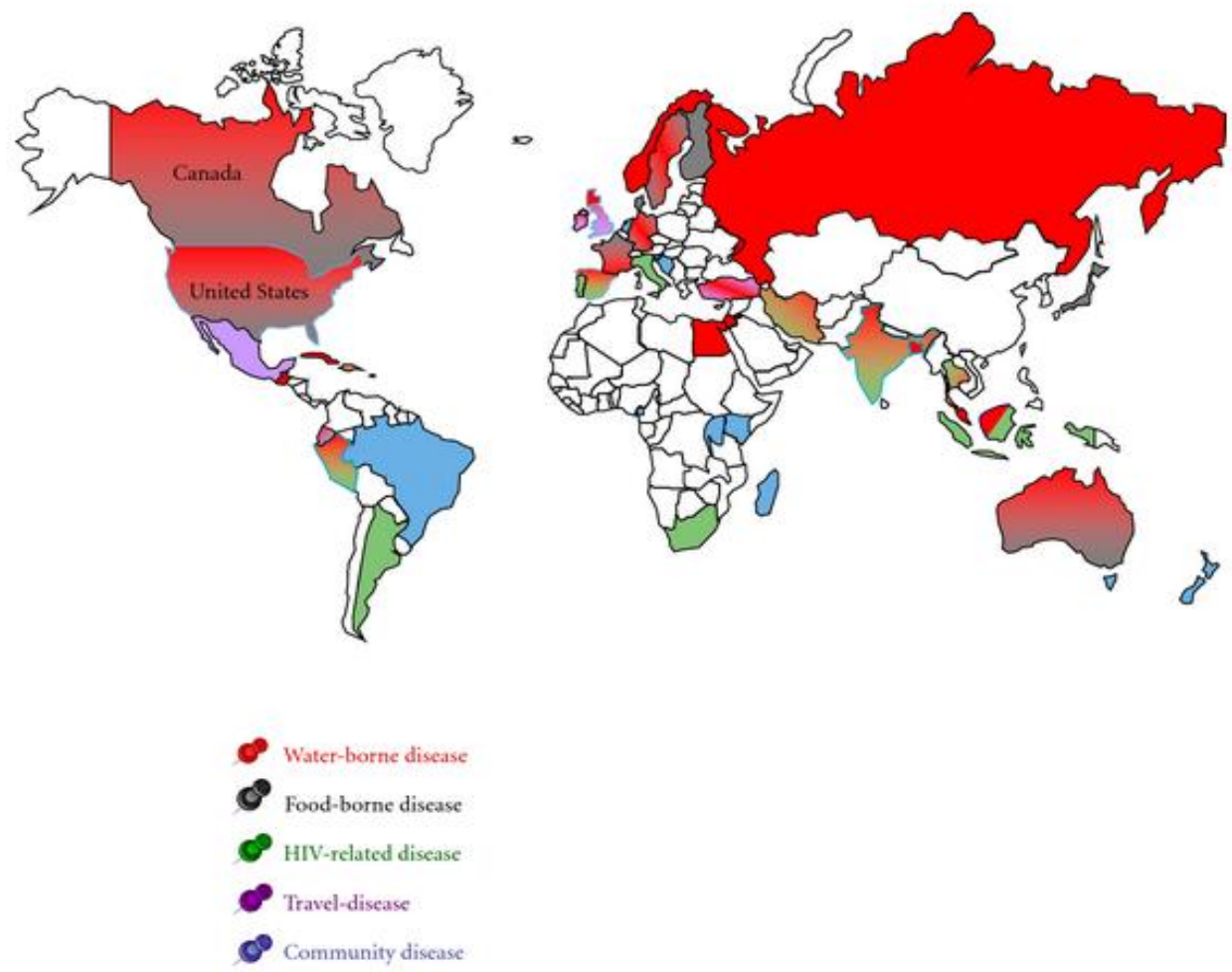

Figure 1.4.: Global sporadic outbreaks of cryptosporidiosis between 1998 and 2008. Source: Putignani \& Menichella (2010).

While other pathogens cause moderate-to-severe diarrhea in children, Cryptosporidium is amoung the most prevalent in developing countries. For example, the Global Enteric Multicenter Study (GEMS) (2007-2011) was conducted in 7 diverse and highly-burden sites in Asia and Africa, including Gambia, Kenya, Mali, Mozambique, Bangladesh, India and Pakistan. The 
comprehensive study assessed moderate-to-severe diarrhea in approximately 22,000 children under the age of 5 and concluded that cryptosporidiosis is the second largest cause of diarrheal death in infants (second to Rotavirous) (Kotloff et al., 2012). Similar conclusions were drawn from the Malnutrition and the Consequences for Child Health and Development (Mal-ED) study (2009-2014), where enteric pathogens were analyzed in over 2100 children from Sub-Saharan Africa, Asia, and South America including South Africa, Tanzania, India, Bangladesh, Pakistan, Nepal, Brazil and Peru. The study concluded that Cryptosporidium was ranked 5 of 22 detected pathogens in children under the age of 24-months (Platts-Mills et al., 2015). Overall, the largescale GEMS and Mal-ED investigations demonstrate that Cryptosporidium plays an integral role in causing illness and mortality in children of low-income countries.

Apart from childhood infections, cryptosporidiosis is a burden amoung the general population and visiting travelers of developing countries. For example, Russian and Bulgarian drinking waters have tested positive for Cryptosporidium from municipal tap and well sources (Karanis et al., 2006). Furthermore, cryptosporidiosis has been diagnosed in returning travelers who visited Russia (Jokipii, Pohjola, \& Jokipii, 1983), the Caribbean (Ma, Kaufman, Helmick, d'Souza, \& Navin, 1985), Egypt, Mauritius (Gatti, Cevini, Bruno, Bernuzzi, \& Scaglia, 1993), Pakistan (Flegg, 1987) and Mexico (Nair et al., 2008). As such, the WHO added cryptosporidiosis to its Neglected Diseases Initiative database in 2004. The database consists of parasitic, bacterial and viral diseases that occur mainly in developing countries, and are connected to climate, poverty and inaccessible services (Savioli, Smith, \& Thompson, 2006). Cryptosporidiosis in particular, is often linked to remote and rural communities, urban overcrowded cities and natural disaster zones (Damiani et al., 2013; Putignani, \& Menichella, 2010; Snelling et al., 2007). Furthermore, food and poor sanitation are typical sources of enteric-pathogen exposure in developing 
countries; however, due to inaccessible technologies and healthcare infrastructure, these sources are under reported in relation to cryptosporidiosis (Ashbolt, 2004).

Last, cryptosporidiosis is a burden in industrialized countries. Putignani and Menichella (2010) composed a global review, which investigated 71 sporadic outbreaks of cryptosporidiosis reported between 1998 and 2008. The study determined that most outbreaks were waterborne (56.3\%), and infected both adults and children. In addition, most outbreaks were concentrated in developed countries, including the US, Canada, Australia, UK and Ireland. Notably, these findings do not reflect the global underreporting of cryptosporidiosis; however, they do disclose that the parasite is a burden even in high-income countries. Cryptosporidiosis in developed countries are often associated with small drinking water communities, which sometimes employ primitive water treatment processes that are incapable of eradicating Cryptosporidium oocysts or have regular failures that are not addressed (Pons et al., 2015). In addition, waterborne outbreaks are often associated with recreational water-use (e.g., amusement parks and swimming pools) (Putignani \& Menichella, 2010). Conversely, over the past 30 years, improved technologies, water-quality management practices, and monitoring of recreational and drinking water catchment areas have resulted in reduced cryptosporidiosis infections in industrialized nations (Bridge et al., 2010; Lake et al., 2007). Unfortunately, the burden and consequences of cryptosporidiosis falls heavily on developing countries in the absence of such interventions.

\subsection{Cryptosporidium in the Caribbean}

Only a few publications have investigated Cryptosporidium in the Caribbean. All studies were conducted in the capital cities and surrounding regions of Cuba, Jamaica and Haiti. Most investigations focused on the clinical manifestation of cryptosporidiosis in human populations. 
More recently, research has shifted to focus on existing species/genotypes and subtypes of Cryptosporidium, along with surface and groundwater prevalence; however, this data is limited.

The first publication dates back to Cuba in 1989. Delfin, Sanjurjo, Findlay and Gordeeva (1989) collected fecal specimens from 200 children—ages 2 months to 4 years—experiencing diarrhea at the Children's Hospital of Havana City. Various enteric pathogens were assessed while using a formol-ether sedimentation method, followed by modified Ziehl-Neelsen microscopy. Cryptosporidium was identified in $8 \%$ of patients, which was the second most common pathogen detected. Moreover, Cryptosporidium was found most frequent in the youngest patients. Subsequent investigations in Cuba confirmed this correlation as Cryptosporidium infections were identified amoung young children, from pre-school to 8 years, in the provinces of Ciego de Ávila and Havana (Núñez et al., 2003a; Núñez et al., 2003b; Pelayo et al., 2015). Similarly, Lindo et al. (1998) was the first pilot study to investigate cryptosporidiosis in Jamaica. A total of 328 stool samples from patients less than 1 to 81 years of age were collected from the University Hospital of the West Indies (UHWI) in Kingston. The study determined that $4 \%$ of samples tested positive for C. parvum, of which highest prevalence was observed amoung children under the age of 2 . Also, C. parvum infections occurred in 3 adults who had HIV.

In Haiti, Cryptosporidium research was becoming increasingly popular, especially after the January 2010 earthquake in the capital city, Port-au-Prince (Damiani et al., 2013). The aftermath of earthquakes is known to displace populations and change environmental conditions, resulting in communal vulnerability to existing pathogens, including Cryptosporidium (Devane, Moriarty, Wood, Webster-Brown, \& Gilpin, 2014; Marahatta, 2015). In Haiti, Cryptosporidium research is also relevant because the parasite was previously deemed the most frequent cause of diarrhea in the country - responsible for $17 \%$ of acute diarrhea in infants under the age of 2 , and $30 \%$ of 
severe diarrhea in HIV-infected patients (Pape et al., 1987; Pape \& Johnson, 1993). High correlations have also been linked to low socio-economic conditions and over crowdedness in large Haitian cities (Raccurt et al., 2006).

In terms of Cryptosporidium species and genotypes, Haiti was the first to investigate this in the Caribbean. Raccurt et al. (2006) analyzed 1529 stool samples from patients with diarrhoea at the GHESKIO Centres and the University Hospital in Port-au-Prince. Approximately, 10\% of adults and children tested positive for cryptosporidiosis via modified Ziehl-Neelsen microscopy. Amoung this group, a genotyping tool, referred to as restriction fragment length polymorphism (RFLP)-PCR was used to determine a species population consisting of $C$. hominis (59\%), $C$. parvum (38\%) and C. felis (3\%) from 69 isolates. The data confirmed a high prevalence of $C$. hominis in young patients, whereas immunocompromised patients were equally infected by both human and animal genotypes. That same year, Ngouanesavanh et al. (2006) investigated Cryptosporidium spp. population in 49 Haitian patients, also from the GHESKIO Centres and University hospital of Haiti. C. hominis and C. parvum were detected in 3 and 2 patients, respectively, which was interpreted as epidemic clonality. In other words, the low genomic diversity suggests that the same or similar isolates of Cryptosporidium were continuously involved in either geographical or temporal outbreaks in Haiti.

In Jamaica, advanced molecular research was performed by Gatei et al. (2008). Stool samples were collected from HIV-infected adults from various hospitals in Kingston, Jamaica. A total of 35 individuals tested positive for Cryptosporidium. PCR and gene sequencing techniques were used to determine a species population of C. hominis (69\%), C. parvum (20\%), C. felis (3\%), C. canis (3\%), and a C. felis and C. hominis (3\%) coinfection. In addition, sequencing of the gp60 gene determined that $C$. hominis specimens were subtypes IbA10G2 (88\%) and IeA12G3T3 
(12\%). Whereas, C. parvum specimens were subtype IIcA5G3d (Gatei et al., 2008), which is generally associated with patients who experience more severe symptoms of cryptosporidiosis (Iqbal, Lim, Al Mahdy, Dixon, \& Surin, 2012). In Cuba, gp60 analysis of Cryptosporidium infections was also performed. Stool samples from 28 children (ages 2-8 years) were assessed, of which 10 C. hominis subtypes were determined, but no $C$. parvum. The subtypes identified were from the Ia, Ib and Id families, demonstrating considerable diversity.

Finally, research pertaining to Cryptosporidium detection from water sources is very limited in the Caribbean. Damiani et al. (2013) is the only available publication to have investigated the prevalence of Cryptosporidium spp. in surface and groundwater sources. A total of 16 water samples were collected from Port-au-Prince and Cap Haïtien, Haiti. Cryptosporidium was detected in 15 samples via microscopy and PCR, but only C. parvum was sequenced in all positive samples. The study noted that it was difficult to determine mixed populations of Cryptosporidium spp. from environmental samples, possibly due to inhibitory factors and the lack of DNA present.

\subsection{Methods for Detecting and Genotyping Cryptosporidium spp. from Environmental Samples}

\subsubsection{Polymerase Chain Reaction (PCR) and Gene Sequencing Applications Proceeding the} 1993 Cryptosporidium Outbreak in Milwaukee

One of the most influential achievements to rise from the Milwaukee outbreak was the application of PCR for rapid detection and gene sequencing of Cryptosporidium spp. Johnson et al. (1995) was the first to publish a conventional PCR protocol, which tested 14 preserved watersamples from the Milwaukee outbreak. The protocol was developed to improve the specificity of 
oocysts detection in drinking-water sources. Briefly, samples were centrifuged, washed, and subjected to a novel six-cycle freezing and thawing step, to extract RNA from the interior part of the oocysts, targeting the $18 \mathrm{~S}$ rRNA gene prior to running PCR. Once completed, the study confirmed the presence of Cryptosporidium in $21.4 \%$ ( $n=3)$ of Milwaukee's samples, which was initially confirmed via microscopy. The practicality of PCR was found comparable to microscopic methods, as it could simultaneously deliver rapid analysis of many samples at a relatively low cost (Johnson et al., 1993; Morgan \& Thompson, 1998; Rochelle, De Leon, Stewart, \& Wolfe, 1997a). Therefore, Johnson et al. (1995) developed a PCR protocol following the Milwaukee outbreak, which offered a new way to confirm oocysts detection in water. Since then, many researchers adopted and improved the efficiency of PCR, specifically for Cryptosporidium detection and gene sequencing from water sources (DiGiorgio, Gonzalez, \& Huitt, 2002; Jiang, Alderisio, Singh, \& Xiao, 2005; Li et al., 2012; Morgan, Constantine, Forbes, \& Thompson, 1997; Rochelle, De Leon, Stewart, \& Wolfe, 1997b; Stinear, Matusan, Hines, \& Sandery, 1996; Sulaiman, 1998; Xiao, Alderisio, Limor, Royer, \& Lal, 2000; Zhou, Singh, Jiang, $\&$ Xiao, 2003).

Over the ensuring decades, PCR technology developed, and researchers began to use variations of the technique to identify species and genotypes of Cryptosporidium isolates from archived Milwaukee samples. This laid the foundation for source-water tracking approaches tailored specifically to monitor the epidemiology of Cryptosporidium. For instance, the outbreak was initially suspected to be caused by a cattle genotype of $C$. parvum - a species that infects both humans and animals (Gradus, 2014; Mac Kenzie et al.,1994). Dairy cattle are major reservoirs of C. parvum (O'Handley et al., 1999), and in 1993, Wisconsin was the second largest dairyproducing state in the country (United States Department of Agriculture [USDA], 2017). Human wastewater and local slaughterhouses were also suspected to be potential sources of the outbreak 
(Mac Kenzie et al, 1994). However, in 1998, retrospective analysis of clinical isolates preserved from the Milwaukee outbreak confirmed that most genotypes were in fact anthropocentric (Sulaiman, 1998).

The human-type isolates were confirmed by Sulaiman (1998). The study used RFLP-PCR to determine inter-species variations of Cryptosporidium. The technique could differentiate human from animal isolates, which verified that the genotypes from the outbreak were mostly human. Nearly a decade after the outbreak, Zhou, Singh, Jiang and Xiao (2003) also used RFLP-PCR to analyze Milwaukee wastewater, which confirmed similar results. In the study, C. parvum (human genotype-1) —now known as C. hominis—was found indistinguishable from the outbreak isolates, which indicated there had been continuous transmissions of human cryptosporidiosis in the city. In addition, the study used nested-PCR to validate these findings, where three or more sets of primers were used to identify Cryptosporidium spp. rather than one. Today, RFLP-PCR and nested-PCR are common applications for Cryptosporidium detection and genotyping from water sources (Mayer \& Palmer, 1996; Nichols, Campbell, \& Smith, 2003; Nikaeen, Mesdaghinia, Tehrani, Rezaeian, \& Makimura, 2005; Sturbaum et al., 2001).

Further explorations of PCR and gene sequencing occurred post 1993. For example, reverse transcription-PCR (RT-PCR) of the mRNA hsp70 gene is used to determine viability of oocysts detected from water (Hallier-Soulier \& Guillot, 2003; Rochelle et al., 1997a; Stinear et al., 1996). Cell culture-PCR (CC-PCR) is used for in vitro culturing of Cryptosporidium within HCT-8 cells to determine infectivity (Di Giovanni et al., 1999; Keegan, Fanok, Monis, \& Saint, 2003; Le Chevallier et al., 2003; Rochelle et al., 1997a). Gene sequencing and phylogenetic analysis of PCR products are used to confirm positive detections, as well as to source track Cryptosporidium spp. to their origin (Prystajecky, Huck, Schreier, \& Isaac-Renton, 2014; Yang 
et al., 2008). Moreover, sequencing of the gp60 gene is used to determine the subtypes of Cryptosporidium spp. - a common practice that assess the genetic diversity and transmission dynamics of the parasite (Stensvold, Beser, Axén, \& Lebbad, 2014). Last, PCR applications collectively, are used to evaluate existing primers that target different Cryptosporidium genes or different loci of the same genes (Guy, Payment, Krull, \& Horgen, 2003; Rochelle et al., 1997a). Overall, these studies continue to build on existing databases, which have produced many new primers that are species specific. Therefore, the application of PCR for Cryptosporidium detection was first put in an epidemiological context when determining the source of contamination of the notorious Milwaukee outbreak. Since then, the same PCR protocols have been referenced globally in many Cryptosporidium studies, including surface water, storm water and wastewater applications (Li et al., 2012; Mayer \& Palmer, 1996; Ruecker et al., 2005; Xiao et al., 2000; Yang et al., 2008).

Although PCR and gene sequencing applications are useful for identifying Cryptosporidium spp., many researchers have uncovered limitations pertaining to specificity and sensitivity. Sulaiman, Xiao and Lal (1999) repeated past PCR protocols used to detect C. parvum between 1994 and 1998. The study sequenced the DNA of PCR products and revealed that the primers used in many protocols amplified the DNA of closely related Eimeria species, producing falsepositives. In addition, Sturbaum et al. (2001) used a nested RFLP-PCR technique to evaluate its sensitivity for oocysts detection and genotyping from water sources. The study determined an average detection rate of $38 \%$ for a single oocyst, whereas greater than $90 \%$ for five or more oocysts. Furthermore, researchers observed reduced sensitivity of PCR applications when analyzing oocysts from environmental samples due to high-level of inhibitors and low DNA yield (Adamska, Leonska-Duniec, Sawczuk, Maciejewska, \& Skotarczak, 2012; DiGiorgio et al., 2002; IOM, 2009). Overall, PCR amplifications and sequencing techniques are limited due to 
non-specific primers, reduced sensitivity of samples that contain low number of oocysts (DNA), and the presence of inhibitors.

\subsubsection{US EPA Method 1623: Filtration/Immunomagnetic Separation (IMS)/ Immunofluorescence Assay (IFA) Microscopy}

Given the uncertainties of PCR, microscopic methods have been standardized to reduce the overall subjectivity of Cryptosporidium oocysts identification. The US Environmental Protection Agency (EPA) Method 1623 is one of the most widely accepted protocol used to detect Cryptosporidium from aqueous matrixes via Immunomagnetic Separation (IMS)immunofluorescence assay (IFA) microscopy. The mandated protocol was developed following the Milwaukee outbreak, and it is based on laboratory recommendations convened by a panel of experts.

The method involves four main steps: 1) raw water is pumped from the site; 2) samples are concentrated via filtration and centrifugation; 3) IMS isolates any oocysts contained within the samples from debris and non-targeted microorganisms; and 4) enumeration is performed via IFA microscopy, and further characterization is performed by 4',6-diamidino-2-phenylindole (DAPI) staining and differential interference contrast (DIC) microscopy as shown in Figure 1.5. (US EPA, 2005).

Apart from microscopic analysis, the IMS component of Method 1623 is used to remove inhibitors prior to running PCR (Jiang et al., 2005; Sturbaum et al., 2002). Researchers also perform the method for screening, as well as for verification of PCR positive detections (Di 
Giovanni, 1999; Hallier-Soulier \& Guillot, 2003; Krometis, Characklis, \& Sobsey, 2009; Lowery, 2001; Sturbaum et al., 2002).
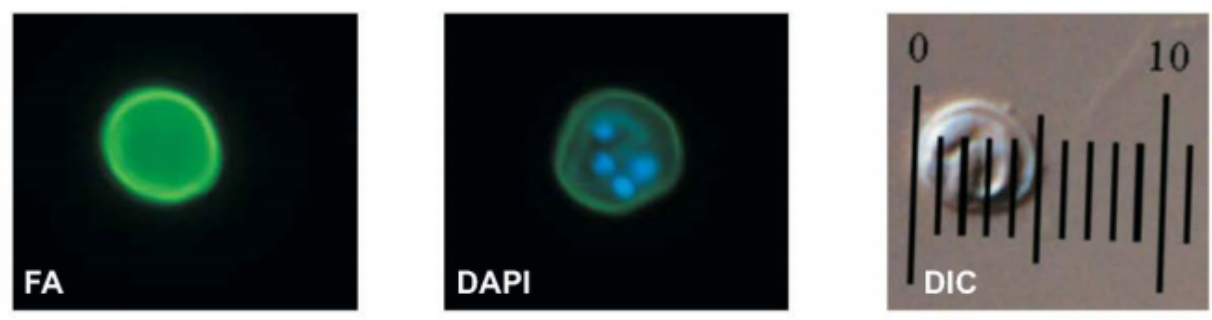

Figure 1.5.: Cryptosporidium oocysts under IFA, DAPI and DIC microscopy. Source: US EPA (2012).

Overall, Method 1623 has demonstrated high oocyst-recovery rates and improved specificity. Roser, Ashbolt, Ongerth, \& Vesey (2002) designed a proficiency test, where 5 experienced laboratories used Method 1623 to recover seeded oocysts from water. Satisfactory performances obtained oocyst-recovery rates between 45 and $81 \%$. These results are substantially higher than the earlier EPA Information Collection Rule (ICR) method, which typically obtains lower recovery rates between 7 and 11\% (Clancy, Gollnitz, \& Tabib, 1994; Hsu, Huang, \& Yeh, 2002). Furthermore, Method 1623 has shown to improve the specificity of oocysts identification by providing specific guidelines to assert positive detections as outline in section 3.2.3. This reduces the overall subjectivity of results.

In contrast, the downfall of Method 1623 is its high overhead costs associated with equipment and labour as demonstrated in Table F.I., Appendix F. Also, the method requires a high degree of analytical expertise to properly assert positive detections under microscopy (Clancy et al., 1994; Jakubowski et al., 1996; Le Chevallier, Norton, Siegel, \& Abbaszadegan, 1995; Rochelle et al., 1997a). This is mainly because the monoclonal antibody used for staining sometimes cross- 
reacts with species of algae (Waterborne Inc., 2010). Moreover, naturally occurring factors such as turbidity and low oocysts levels, generate inconsistent recovery rates (DiGiorgio et al., 2002). All of these factors highlight the difficulty of using the method to determine oocysts concentration and make predictions about the environment. The only way to obtain a comprehensive representation of oocysts in natural waters is to filter multiple samples at a single site during different times of the day. Performing this step requires specialized equipment, which is too expensive to adopt in most countries for regular surveillance (Hsu et al., 2002).

\subsubsection{Modified Acid-Fast (MAF) Staining: White Light Microscopy}

Prior to IFA microscopy, the gold standard for Cryptosporidium oocysts detection from fecal samples was MAF staining, in which oocysts are stained a pinkish-red colour, contrasting to a

dark green or blue counter stain (Figure 1.6.). Kinyoun's Carbol Fuchsin (KCF) is a popular stain used for the procedure, developed in Paik and Suggs (as cited in Ma \& Soave, 1983). Since then, the CDC has developed its own MAF protocol, which incorporates $\mathrm{KCF}$ for identifying Cryptosporidium oocysts from stool samples. An alternative stain is Ziehl-Neelsen; although, $\mathrm{KCF}$ does not require a pre-heating step (CDC, 2016b), thus it is presumed a faster diagnostic tool.

In terms of efficiency, MAF staining is considered less specific and sensitive than IFA (CDC, 2016c). Nonetheless, MAF remains the most common diagnostic tool used for Cryptosporidium detection in fecal samples. This is because MAF reagents are inexpensive and do not require specialized equipment. Arguably, if MAF is executed appropriately (e.g., sufficient staining time and analysis), then it can perform just as well or even better than IFA (Cole, 1997). 
In comparison to non-microscope methods such as ELISA and PCR, MAF is less expensive; however, its labourious and consumes a lot of time (Rekha, Puttalakshmamma, \& D'Souza, 2016). In addition, MAF is prone to error since Cryptosporidium oocysts are small and can be easily confused with artifacts (Tahvildar-Biderouni \& Salehi, 2014). It is also possible to mistaken Cryptosporidium oocysts for other coccidian species including Cystoisospora, Cyclospora, yeast and mould. These species are generally larger than Cryptosporidium, but they appear similar and often take up the same pinkish red colour (Rekha et al., 2016). It is also possible that some oocysts do not stain due to over exposure to decolourizer. Overall, MAF requires microscopic expertise, thus it is more prone to error than ELISA and PCR methods.

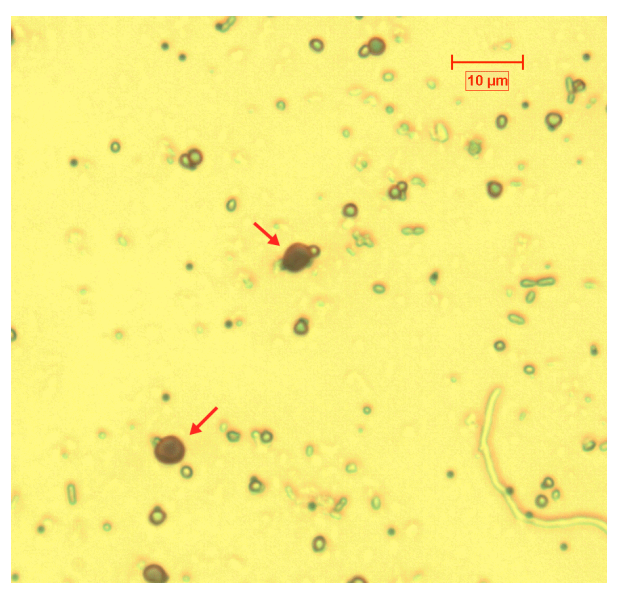

Figure 1.6.: MAF stained, positive control Cryptosporidium oocysts from cattle feces captured under white light microscopy. The Ward's ${ }^{\circledR}$ Chemistry Acid-Fast Stain Kit was used. The carbol fuchsin stained two oocysts a deep pink colour. Oocysts have a well-formed wall with diameter of approximately 4-5 $\mu \mathrm{m}$. Magnification, 100X with immersion oil. Image curtesy of Simmoy Noble, Ryerson University.

\subsubsection{Enzyme-linked Immunosorbent Assay (ELISA) Test}

ELISA is an immunoassay, typically used for clinical and veterinary detection of Cryptosporidium from stool samples. The technique uses either antigens or antibodies to react 
with the presence of the parasite. This is performed by transferring stool specimens into 96microwell plates that are coated with the species' antigens or anti-Cryptosporidium antibodies. The presence of Cryptosporidium is determined through a colour change when the antigens/antibodies from stool react to the coated surfaces of the plastic wells (Diagnostic Automation, Inc., 2003; Vohra, Sharma, \& Chaudary, 2012).

Utilization of ELISA offers an accurate, fast and easy-to-read method. Studies have determined that the sensitivity of ELISA is approximately $87-93 \%$, while the specificity is $95-99 \%$ (Ghoshal, Jain, Dey, \& Ranjan, 2018; Rosenblatt and Sloan, 1993). Moreover, ELISA methods are easy to perform because they do not require observation of intact oocysts. Thus, proficiency tests have shown to produce results comparable to that of experienced microscopists (Diagnostic Automation, Inc., 2003)

While ELISA has proven to be a reliable method, a major drawback is its high cost (Vohra et al., 2012). Also, like many other antigen-based diagnostic tests, ELISA fails to differentiate disease carriers who are shedding oocysts, from those who have recently recovered. For example, ELISA kits designed to detect antibodies specific to Cryptosporidium spp. have shown positive for patients who recently recovered from confirmed infections, but still have detectable antibodies in their stool (Campbell \& Current, 1983). Furthermore, immunoassays that target Cryptosporidium antigens have shown to produce false-negatives due to low parasite density in the fecal specimens (Vohra et al., 2012).

\subsection{Specific Water Parameters that Influence the Morphology, Survival and Dispersion of Cryptosporidium Oocysts}


Various water parameters including $\mathrm{pH}$, dissolved oxygen (DO), E. coli/fecal coliforms, dissolved organic carbon (DOC), temperature, water hardness and salinity, influence the survival and dispersion of Cryptosporidium oocysts. These parameters either shield or change the morphological characteristics of oocysts, as well as produce microclimates in which they flourish. Here, specific details about how each parameter influences the longevity of oocysts, and whether they can be used to indicate risk of contamination is provided.

$\mathrm{pH}$ is used to measure the acidity and basicity of water. The reaction between water molecules and other compounds result in imbalances, where water becomes more acidic (more $\mathrm{H}+$ ) or basic (more OH-) (Behar, 1997). In relation to Cryptosporidium, $\mathrm{pH}$ influences the surface charge of oocysts. Naturally, oocysts have a neutral surface charge when water is maintained at a neutral $\mathrm{pH}$ level. However, the presence of natural organic matter $(\mathrm{NOM})$ in water lowers $\mathrm{pH}$, resulting in a negative surface charge of oocysts. This in turn effects their electrostatic binding capacity to other material present in water (Dai \& Hozalski, 2002). In addition, studies have investigated the correlation between $\mathrm{pH}$ and oocysts viability and determined that the number of viable oocysts slightly decreases at $\mathrm{pH}$ levels $<4$ or $>11$ (Jenkins, Bowman, \& Ghiorse, 1998; Kniel et al., 2003). In contrast, major viability losses occur at $\mathrm{pH} \leq 1$ or $\geq 13$ (Campbell, Robertson, \& Smith, 1992). Therefore, under extreme acidic and basic conditions, $\mathrm{pH}$ significantly decreases the number of viable oocysts. Since freshwater and saltwater typically maintain a pH between 6 and 8.1 (National Geographic Society, 2017; Peacock et al., 2004), pH is not an effective parameter used to predict the risk of Cryptosporidium contamination from environmental sources.

DO measures the amount of oxygen gas in water. In general, high DO levels are consistent with healthy ecosystems; whereas low DO levels are consistent with oxygen-consuming coliforms and organic waste, including sewage and oil biproducts, which are locally referred to as 
'dundar'. Sente et al. (2016) collected water from the Queen Elizabeth Protected Area, Uganda, and observed a strong negative correlation $(p<0.05)$ between DO and the presence of Cryptosporidium. Similarly, Hogan et al. (2012) analyzed fecal and water samples from California wetlands and observed the same negative correlation between DO and protozoal counts $(p<0.0001)$, which included Cryptosporidium spp. Thus, low DO concentrations in water indicates the presence of excess fecal coliforms and organic waste. Since Cryptosporidium spp. derive from the same sources (e.g., sewage and agricultural runoff), DO could potentially be used to predict oocysts contamination.

For the same reasoning, E. coli and fecal coliforms are also potential indicators; however, numerous studies have shown that these parameters sometimes fail to correlate with Cryptosporidium (Harwood et al., 2005; Lalancette et al., 2014). Therefore, monitoring a selective group of organisms rather than a single surrogate pathogen, is more likely to predict the presence of oocysts in water.

DOC can be defined as the total organic material dissolved in water. DOC is calculated by measuring the amount of organic matter that passes through a filter. Water treatment facilities have found that low DOC correlates with the log reduction of Cryptosporidium oocysts (Considine, Dixon, \& Drummond, 2002; Xagoraraki \& Harrington, 2004). This is due to two possible factors. First, the presence of DOC causes the surface-layer proteins of oocysts to collapse, resulting in increased adhesion to organic matter (Plummer, Edzwald, \& Kelley, 1995). Second, DOC inhibits the inactivation of oocysts by UV light. Therefore, high DOC content (e.g., algae) prevents the penetration of the sun's rays, which normally would denature oocysts (King, Hoefel, Daminato, Fanok, \& Monis, 2008). All in all, DOC influences the presence of Cryptosporidium oocysts in water by enhancing their adhesion to organic material, which in turn 
shields them from the sun's UV rays. For this reason, DOC may be a sufficient water parameter used to predict Cryptosporidium contamination.

Water temperature is influenced by air temperature, sunlight, inflow of groundwater, terrestrial runoff and turbidity. Current research suggests that oocysts maintain infectivity for at least 3 months when stored in water temperatures between 4 and $15^{\circ} \mathrm{C}$ (King, Keegan, Monis, \& Saint, 2005). At $20^{\circ} \mathrm{C}$, Cryptosporidium oocysts can survive for about 6 months, while at a slightly higher temperature of $25^{\circ} \mathrm{C}$, oocysts inactivate after 3 months (Fayer et al., 1998). This means that the ideal temperature for Cryptosporidium spp. to thrive is approximately $20^{\circ} \mathrm{C}$. Under extreme temperatures however, oocysts lose their infectivity. For example, in hot water, inactivation of oocysts occurs at $71.7^{\circ} \mathrm{C}$ (after 15 seconds) (Harp, Fayer, Pesch, \& Jackson, 1996), whereas in frozen water, oocysts inactivate at $-70^{\circ} \mathrm{C}$ and $-20^{\circ} \mathrm{C}$ at 1 and 24 hours, respectively (Fayer \& Nerad, 1996). Therefore, water temperatures influence the survival of Cryptosporidium oocysts, enabling them to thrive under optimal temperatures, and deactivate in extreme hot or cold water. Depending on climate, water temperature can be used to predict the seasons, in which oocysts are expected to be most prevalent.

Hard water contains high concentrations of calcium and magnesium, which dissolves as water percolates limestone $\left(\mathrm{CaCO}_{3}\right.$ or $\left.\mathrm{MgCO}_{3}\right)$. Salinity also contributes to water hardness. For example, the total water hardness of freshwater is generally between $10 \mathrm{mg} / 1$ to $250 \mathrm{mg} / 1(0.01$ ppt to $0.25 \mathrm{ppt}$ ) as $\mathrm{CaCO}_{3}$; whereas the hardness of seawater is usually greater than $6000 \mathrm{mg} / 1$ (6 ppt) (Vernier Software \& Technology, n.d.). Studies have found that Cryptosporidium oocysts can persist in hard waters for several months. Modini, A. Pizarro, M. Pizarro and Zerbatto (2016) investigated the viability of oocysts in artificial saltwater (salinity: $35 \mathrm{ppt}$ ) maintained at $20^{\circ} \mathrm{C}$. Through in vitro excystation, it was determined that a large proportion of oocysts was viable 
$(28.6 \pm 9.79 \%)$ in seawater after 16 weeks. A slightly lower longevity was determined in seawater by Fayer, Graczyk, Lewis, Trout, \& Farley (1998). In the study, Cryptosporidium oocysts was inoculated in artificial seawater (salinity: $10-30 \mathrm{ppt}$ ) maintained at $20^{\circ} \mathrm{C}$, then bio-assayed in 5 to-7-day old mice to assess viability. It was determined that oocysts were viable at salinities of 0$10 \mathrm{ppt}, 20 \mathrm{ppt}$ and $30 \mathrm{ppt}$ for 12 weeks, 4 weeks, and 2 weeks, respectively. Therefore, water hardness in freshwater is usually an insignificant indicator of Cryptosporidium; however, in coastal region and islands, where there is an influx of brackish water from the sea, water hardness may elevate to $>30 \mathrm{ppt}$. In such cases, water hardness may be an important parameter to measure for predicting Cryptosporidium viability.

\subsection{Cryptosporidium-Biofilm Association}

\subsubsection{Aquatic Biofilms}

Aquatic biofilms also influence the survival and transport of Cryptosporidium oocysts. A biofilm is an aggregation of microorganisms, oriented in a highly efficient and stable system. Biofilms settle in water columns and adhere to many surfaces such as rocks and sediments of stream beds (Langmark, Storey, Ashbolt, \& Stenstrom, 2005; Stoodley, Sauer, Davies, \& Costerton, 2002). The biofilm structure is very complex, usually consisting of bacteria embedded in a polymeric matrix (Flemming \& Wingender, 2010). These consist of numerous components including microbial cells such as bacteria, archaea and protozoa (2-5\%), water (major component that equals to about 97\%), polysaccharides (1-2\%), extracellular proteins (1-2\%), DNA and RNA (1$2 \%$ ), and ions (Takhistov \& George, 2004). All components of the biofilm are embedded in a self-produced extracellular matrix that helps to protect microorganisms from harsh external forces. 
Aquatic biofilm formation results from an initial attachment of a free microorganism to a surface. This inception is aided by the Van der Waal forces of attractions and hydrophobic effects (Kolter, 2015). Once the initial colony is fixed, the bacteria permanently anchor themselves using adhesion structure-like pili (Ryder \& Mascarenhas, 2007). Increased hydrophobicity can also make the microorganisms fasten themselves together to enduringly form a colony. During attachment, the microorganisms secret an extracellular polysaccharide substances (EPS) that encloses the microorganisms, creating a biofilm, which thereafter grows in size due to the constant addition of microorganisms (Bonnineau, Tlili, Faggiano, Montuelle, \& Guasch, 2013; Stoodley et al., 2002). Throughout this entire process, individual cells within the biofilms communicate with one another by means of quorum sensing. This allows for the microbial community to regulate gene expression, thus respond to environmental stimuli, new food sources, and coordinate the recruitment of other microorganisms (Dobretsov, Teplitski, \& Paul, 2009). Once too large, the biofilm undergoes dispersion, in which the microorganisms spread to colonize other areas. This reduces competition for nutrients within the colony (Takhistov \& George, 2004).

\subsubsection{Retention of Cryptosporidium Oocysts in Aquatic Biofilms}

Aquatic biofilms serve as reservoirs for Cryptosporidium oocysts, creating favourble microclimates for their survival and dispersion. Wolyniak DiCesare, Hargreaves, \& Jellison (2012a) inoculated C. parvum oocysts in biofilms, then exposed them to solar radiation. The same solar intensity was simultaneously exposed to free-floating oocysts, and overtime there were about double the number of oocysts attached to the biofilm bases compared to oocysts suspended freely in water. The retention of oocysts in biofilms is facilitated by surface roughness 
and thickness. Through qPCR and COMSTAT analyses, Koh, Clode, Monis and Thompson (2013) determined that the retention of Cryptosporidium oocysts significantly increases $(\mathrm{p}<0.05)$ as biofilms increase in thickness. Other studies have shown a positive correlation between high standard deviation of biofilm thicknesses (indication of surface roughness) and oocysts attachment. These observations of biofilm roughness were suspected to be influenced by seasonal changes (Wolyniak DiCesare, Hargreaves, \& Jellison, 2010; Wolyniak DiCesare, Hargreaves, \& Jellison, 2012b). Furthermore, oocysts can remain intact to biofilms despite the disturbance of flowing water. For example, Searcy, Packman, Atwill, \& Harter (2006) used a flow-cell system and nutrient enrich growth media to observe the capture and retention of $C$. parvum oocysts in $P$. aeruginosa biofilms. After 24 hours, the study found oocysts attached to biofilms (Figure 1.7.), which were released 1 hour later once the flow rate increased by 40 -folds. Overall, substantial evidence indicates that biofilms recruit and retain Cryptosporidium oocysts, protecting them from deactivation via solar radiation and other environmental stressors.

Another benefit that biofilms potentially provide, is a suitable microclimate for oocysts development and reproduction. Within the past decade, there has been an increasing number of studies showing the in vitro extracellular multiplication of Cryptosporidium oocysts in both cell and cell-free cultures (Arrowood, 2002; Clode et al., 2015; Hijjawi, 2004, Hijjawi, Meloni, Ryan, Olson, \& Thompson, 2002). For example, Hijjawi (2010) composed a review on various animal and human host cells that are capable of Cryptosporidium growth, including BFTE, MDCK, BS-C-1, HCT-8 and Caco-2. In addition, Hijjawi, Estcourt, Yang, Monis, \& Ryan (2010) used a cell-free maintenance media to cultivate $C$. hominis and was able to visually observe oocysts' developmental stages without excystation. Finally, Koh et al. (2013) was the first to present significant evidence of extracellular growth of $C$. parvum oocysts in $P$. aeruginosa biofilms under aquatic conditions. The study found a significant increase in $C$. 
parvum of 2-3 folds $(\mathrm{P}<0.001)$ over a 6 -day period as the biofilms matured. Also, the life-cycle stages of C. parvum were identified using confocal microscopy, which showed that the oocysts were multiplying at different life-cycle stages within the biofilms. Therefore, recent studies have demonstrated that Cryptosporidium oocysts can multiply and grow outside hosts. Although, evidence conveying oocysts multiplication within biofilms is relatively new, thus warrants further research.

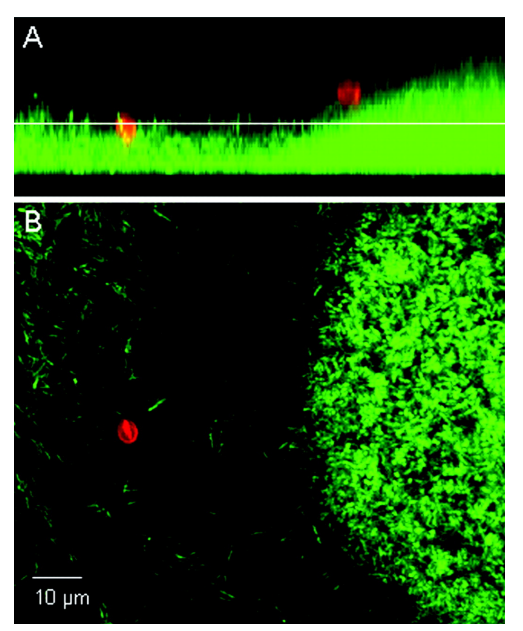

Figure 1.7.: The entrapment of $C$. parvum oocysts (red) in a $P$. aeruginosa biofilm (green). Captured under confocal microscopy using Cy3-conjugated monoclonal antibody to stain oocysts and green fluorescent protein to stain the biofilm. Magnification, 60X. Source: Searcy et al. (2006).

\subsubsection{Biofilm Sampler and Water Monitoring}

Biofilms serve as natural filters in water, hence their analysis could provide critical information about the microbial community that passes along streamlines. Much work has been completed on the recruitment, retention and transportation of pathogens in aquatic biofilms including Cryptosporidium investigations (Luo, Jedlicka, \& Jellison, 2016; Wolyniak DiCesare, Hargreaves, \& Jellison, 2009; Wolyniak et al., 2010; Wolyniak DiCesare et al., 2012b). However, very few studies have focused on using biofilms for water monitoring purposes. 
The biofilm sampler is an apparatus used to monitor various species in water. The innovation permits biofilm development on removable surfaces, which are analyzed to determine microbial composition and behaviour. Original biofilm samplers were used in laboratories, where detachable studs were exposed to flowing water for 2-3-weeks, allowing for mature biofilms to form. Chemical manufacturers would then test these biofilms against various antimicrobial products such as biocides, antiseptics, drain cleaners and antibiotics (Caproco, 2003). Laboratory investigations have since adopted the biofilm sampler technique in flow cell systems, where a medium is continuously pumped through capillary tubing, promoting the attachment and growth of biofilms to a flow cell (glass prism), which is mounted under a microscope. Under these hydrodynamic conditions, the spatial distribution, composition and growth of biofilms can be assessed in real-time (Peterson et al., 2011).

Apart from laboratory investigations, biofilm samplers have a long history in drinking water distribution systems. Inspectors use the device to monitor biofilms in the interior walls of piping networks. The Robbins Device is one of the older and most popular forms of biofilm samplers. The device is placed in a section of a piping network, allowing for biofilms to form on removable coupons that fit in sampling ports. Robbins Devices are typically used to investigate biocidal activity on different surfaces. They are also used to determine the effects of velocity and turbidity on biofilm formation (Azeredo et al., 2016).

In terms of environmental assessment, biofilm samplers have been used to monitor various species in surface water. Fuchs, Katbeh-Bader, \& Alkhateeb (2012) used a biofilm sampler to identify organisms in the Wadi Ar Rumman ravine, Jordan. The samplers were constructed using PVC pipes with glass inserts, providing a flat surface where the biofilms settled and grew (Figure 1.8a.). These biofilms were assessed to determine the impact of heavy metal pollutants on 
species composition. Similarly, Tonetto, Peres and Branco (2012) used a biofilm sampler, which was constructed out of acrylic plaques and glass slides to monitor benthic communities in lotic waters of southern Brazil (Figure 1.8b.). Last, Peacock et al. (2004) used a biofilm sampler constructed out of Teflon tubing loaded with glass wool or Bio-Sep beads to monitor waterquality in a field bioremediation experiment in Tennessee (Figure 1.8c.). These biofilms were analyzed via PCR to determine microbial community structure.

$\mathbf{a}$

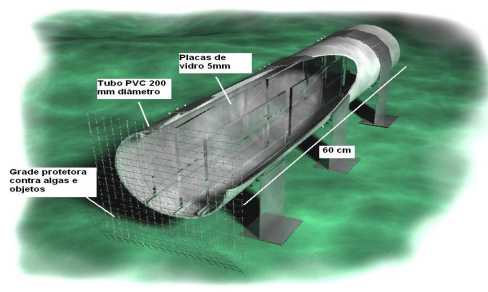

b

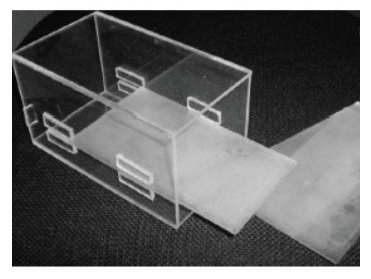

d

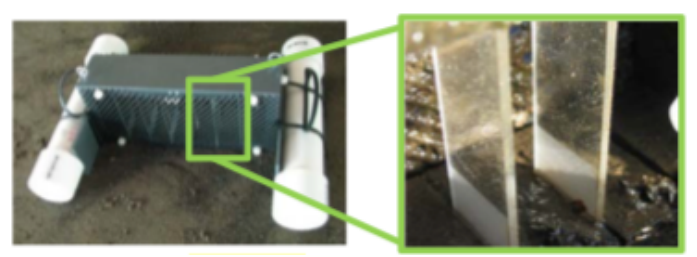

c

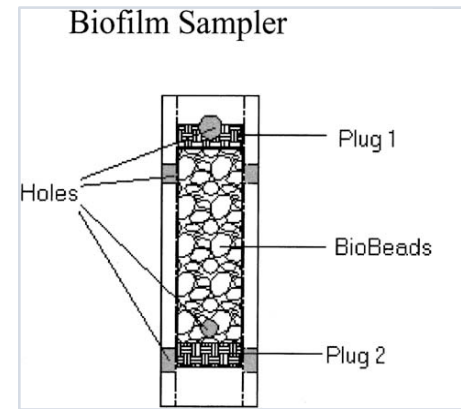

Figure 1.8.: Previous biofilm samplers used for surface water monitoring. (a) to assess the effects of heavy metal pollutants on species composition. (b) to investigate benthic communities in lotic waters (c) to monitor water-quality in a field bioremediation experiment. (d) to monitor the upstream and downstream prevalence of Cryptosporidium oocysts. Sources: Fuchs et al. (2012); Peacock et al. (2004); Philadelphia Water Department [PWD] (2014).; Tonetto et al. (2012).

\subsubsection{Biofilm Sampler Technique for Detection and Molecular Characterization of Cryptosporidium spp. from Surface Water}

Researchers at Lehigh University (Bethlehem, Pennsylvania) coined the "biofilm sampler technique" for in situ monitoring of Cryptosporidium in surface water. In collaboration with the Philadelphia Water Department (PWD), ongoing projects have monitored the upstream and 
downstream prevalence of Cryptosporidium oocysts from various watersheds in Eastern Pennsylvania. The biofilm samplers were constructed out of metal-box frames containing glass microscope slides (Figure 1.8d.). After a 2-3-week period, the biofilms were analyzed for Cryptosporidium oocysts by a partial EPA Method 1623, which excluded the filtration step but abided by the IMS and fluorescent microscopy guidelines (Barnes-Pohjonen, 2012; McLeod, 2011; PWD, 2014).

Significant findings from these projects were compared to Method 1623's filtration to determine the difference between oocysts recovery rates. The biofilm samplers produced $46 \%$ positive (18/39), whereas EPA filters produced 43\% positive (17/40) (Westerling, 2014). In addition, Barnes-Pohjonen (2012) found that Fluorescent in situ Hybridization (FISH) processing of biofilms attached to slides identified more positives than Method 1623, indicating that the biofilm/FISH method was more sensitive and had a lower detection limit. Furthermore, McLeod (2011) used the biofilm sampler technique to genotype Cryptosporidium spp. from biofilm samples. Through nested-PCR and 18S rRNA sequencing, multiple genotypes of C. parvum were identified in the Schuylkill River, and 75\% were associated with human infections. Therefore, previous investigations have demonstrated that biofilm sampling is comparable, and sometimes even more sensitive than Method 1623's filtration. Moreover, it can be used to monitor Cryptosporidium species and genotypes, thus assert public health risk.

\subsection{Summary}

- Cryptosporidium is a protozoan parasite that causes cryptosporidiosis - a diarrheal disease that manifests through the oral-fecal route and can be life threatening to children and immunocompromised individuals. 
- Cryptosporidium is a part of the Apicomplexa phylum. It was traditionally recognized as a coccidian; however, modern research suggests that it is now a gregarine. To date, there are at least 24 species/genotypes, and 65 subtype families of Cryptosporidium.

- Cryptosporidium was first described in 1907. Since then, it has been linked to sickness in livestock, particularly cattle. In 1971, Cryptosporidium gained public health interest after its first reported cases in humans.

- Cryptosporidium is a global burden. Developing countries are at greatest risk due to inaccessible technologies and healthcare infrastructure. In the Caribbean, Cryptosporidium has been detected in human populations and water; however, genomic diversity of species is low. There is also a gap in literature regarding Cryptosporidium spp. detection from environmental sources in the Caribbean.

- Global waterborne outbreaks of cryptosporidiosis have sporadically occurred, leading to major consequences. The 1993 Milwaukee outbreak remains the largest of its kind. Its aftermath prompted research, resulting in enhanced monitoring and detection methods including IFA-microscopy (Method 1623), PCR and gene sequencing.

- The sensitivity and specificity of Cryptosporidium PCR detection and gene sequencing are limited due to environmental inhibitors, false positives yielded from closely related species, and naturally low DNA contained in environmental samples.

- Alternative Cryptosporidium detection methods include: MAF and IFA microscopy, and ELISA antigen test. Although, these methods are also limited due to microscopic subjectivity and the cross-reactivity of stains/antibodies.

- Waterborne transmission is the primary source of human cryptosporidiosis. Various water parameters including $\mathrm{pH}, \mathrm{DO}, \mathrm{DOC}$, hardness and temperature have shown to influence the survival and transport of Cryptosporidium oocysts. Indicator pathogens 
including E. coli and fecal coliforms have shown to be both correlated and inconsistent with Cryptosporidium prevalence.

- Aquatic biofilms have also shown to improve the life expectancy of oocysts by providing suitable microclimates for protection, transportation and potential multiplication.

- Sampling biofilms via the biofilm sampler technique, followed by fluorescent microscopy, PCR and gene sequencing can be useful for monitoring Cryptosporidium spp. in surface water 


\section{Chapter 2: Introduction}

\subsection{Research Aim and Rationale}

Global perspectives of cryptosporidiosis have evolved from that of a rare disease, to a fatal diarrheal disease that can be transmitted between humans, animals, food and water sources. Although surface water is recognized as the primary source of human cryptosporidiosis, regular surveillance in most countries is impractical due to high overhead costs. These expenses are associated with conventional filtration equipment, which have been standardized for use by the US EPA Method 1623. Notably, Method 1623 offers a universal approach that is internationally renowned for regulating Cryptosporidium from surface water. However, the technique only uses fluorescent microscopy, hence does not provide any information about Cryptosporidium at the species or genotype level.

Given the limitations of Method 1623, research presented in this dissertation focused on the development and evaluation of an alternative method referred to as the biofilm sampler technique for microscopic and PCR detection, and molecular characterization of Cryptosporidium spp. from biofilm samples. Based on the literature review, it is understood that biofilms recruit and entrap Cryptosporidium oocysts, thereby facilitate prolonged survival, dispersion and potential multiplication. Therefore, the development of the biofilm sampler approach could aid in the advancement of monitoring and source tracking practices.

It is important to note that the purpose of developing the biofilm sampler technique was not to replace conventional filtration methods; but rather offer an alternative method that could work in conjunction with multiple techniques applied to a set of aquatic sources that are well-known reservoirs of Cryptosporidium spp. Such sources may include biofilm, shellfish, benthic 
macroinvertebrates, which have previously demonstrated to naturally accumulate

Cryptosporidium oocysts (Reboredo-Fernández, Prado-Merini, García-Bernadal, Gómez-Couso, \& Ares-Mazás, 2014; Staggs et al., 2015; Wolyniak et al., 2010).

In addition to biofilm sampling, this dissertation aimed to employ a variety of techniques including MAF microscopy, ELISA antigen testing, PCR and gene sequencing to detect and characterize Cryptosporidium spp. from cattle feces collected within the Black River watershed. The agreement between MAF and ELISA detections was also statistically evaluated. Records were maintained per sample (i.e., age, production type, diarrheal symptoms and farm management) for further interpretation of results. Several studies agree that cattle are major reservoirs of Cryptosporidium spp. that cause zoonosis. Thus, research in the discipline is critical for both human and animal health. It provides informative data, especially in the event of outbreak situations, and may aid in the development of strategies for limiting incidences of cryptosporidiosis throughout the watershed or alike environments.

Finally, the selected region of study was the Black River watershed of Jamaica because most studies in the Caribbean have focused on Cryptosporidium detection from human populations. Very few studies have investigated the population structure of different species and genotypes from environmental sources, including surface waters and animal feces. Specifically, in Jamaica, there is no available research on Cryptosporidium detection from environmental sources. This not only highlights the gap in research related to waterborne and zoonotic transmission, but also the difficulty in detecting and characterizing Cryptosporidium spp. from environmental samples. Based on these observations, further research is necessary to establish a database that provides critical information about existing species and genotypes that are geographically distinct and have the potential to cause zoonosis in the region. 


\subsection{Specific Objectives}

2.2.1. Objective \#1: Method development and evaluation of the biofilm sampler technique for Cryptosporidium oocysts entrapment and recovery efficiency from biofilm suspensions

Method development and evaluation of the biofilm sampler technique is important because it is a cost-effective and practical alternative to conventional filtration. As previously stated, EPA's Method 1623 is the universal method used for Cryptosporidium oocysts monitoring in surface water; however, the technique requires expensive filters that can only monitor oocysts at a specific location, at a single point in time. The biofilm sampler technique on the other hand, has proven to be a cost effective and reusable alternative that can monitor Cryptosporidium oocysts in surface water over a 2-3-week period. The following objective therefore, aimed to design a biofilm sampler that could optimize the growth of biofilms on polycarbonate substrate. The growth surface was hypothesized to produce thicker and rougher biofilms, thereby enhance the entrapment of Cryptosporidium oocysts. Thus, through confocal microscopy and COMSTAT2 analysis, biofilm thickness and roughness were measured and compared to previous studies in relation to biofilm retention of Cryptosporidium oocysts. The objective also aimed to evaluate the recovery efficiency of oocysts seeded into the same biofilms using the IMS-IFA technique and gridded coverslips. All results were statistically evaluated by calculating relative standard deviation (RSD) and are presented in section 4.1 of this submission.

\subsubsection{Objective \#2: Development of PCR assays to optimize Cryptosporidium detection and} genotyping from environmental samples using selected primers

It is widely accepted that PCR assays for Cryptosporidium detection and genotyping from environmental samples are challenging to execute. This is due to low quantities of DNA, as well 
as high prevalence of inhibitors and other microorganisms that interfere with the PCR reaction. This objective therefore aimed to use spectrophotometric analyses and temperature gradient tests to enhance PCR thermocycler conditions, using common CPB-DIAG (Johnson et al., 1995), KLJ (Jellison, Hemond, \& Schauer, 2002), and XIAO nested primers (Xiao et al., 1999). Results from these analyses are presented in section 4.2. of this dissertation, where spectrophotometry was performed to determine adequate primer dilution, as well as the concentration of Cryptosporidium DNA present in each sample type. In addition, temperature gradient tests were performed to determine optimal annealing temperature, which generated the most distinct bands with minimal non-specific binding.

\subsubsection{Objective \#3: Detection and molecular characterization of Cryptosporidium spp. from biofilms in the Black River and tributaries}

The aim of this objective was to use the biofilm sampler technique and PCR methods developed in Objective \#1 and \#2 to detect and characterize Cryptosporidium spp. from biofilms collected from 5 sites in the Black River and tributaries. The main purposes were as follows: 1) to detect Cryptosporidium oocysts via IFA microscopy and differentiate positive detections that have DAPI-visible nuclei; 2) confirm positive detections using a nested-PCR assay; 3) sequence the 18S rRNA and gp60 genes of PCR products and perform phylogenetic analysis to identify Cryptosporidium spp. and potential subtypes that contaminate the water source and thus, pose risk to public health. Results from these analyses are presented in section 4.3. of this submission.

\subsubsection{Objective \#4: Detection and molecular characterization of Cryptosporidium spp. from} cattle feces in the Black River watershed 
This objective aimed to detect and characterize Cryptosporidium spp. from 119 cattle fecal specimens collected from 10 farms within the Black River watershed. This involved the application of MAF microscopy, ELISA, PCR and gene sequencing techniques. The proportion of positives detected per method was correlated with variances between dairy and beef cattle. Gene sequencing and phylogenetic analysis was performed to identify Cryptosporidium spp. and subtypes, as well as to evaluate public health risk based on identified isolates, previously implicated in waterborne and zoonotic infections. Results from these analyses are presented in section 4.4. of this submission.

\subsubsection{Objective \#5: Evaluation of diagnostic agreement between MAF microscopy and ELISA antigen test for the detection of Cryptosporidium from cattle feces}

Since all techniques have sensitivity and specificity limitations, as highlighted in section 1.6., multiple methods were employed to verify positive detections from cattle feces. This objective therefore, aimed to assess the diagnostic (inter-rater statistical) agreement between MAF microscopy and ELISA, by calculating Cohen's kappa coefficient $(k)$. The $k$-values were interpreted based on a well-established classification scheme. This form of statistical analysis took into account the possibility of agreement by chance. PCR was not included in the analysis due to low sensitivity in cattle feces, compared to MAF and ELISA techniques. Results are presented in section 4.4. of this submission. 


\subsection{Summary of Experimental Workflow}

As described in Objective \#s 3-5, multiple techniques were employed to detect and genotype Cryptosporidium spp. Figure 2.1. provides a summary of experimental workflow.

\section{Sample Collection}

2. Concentration and Purificaiton

3. Presence of Cryptopsoridium

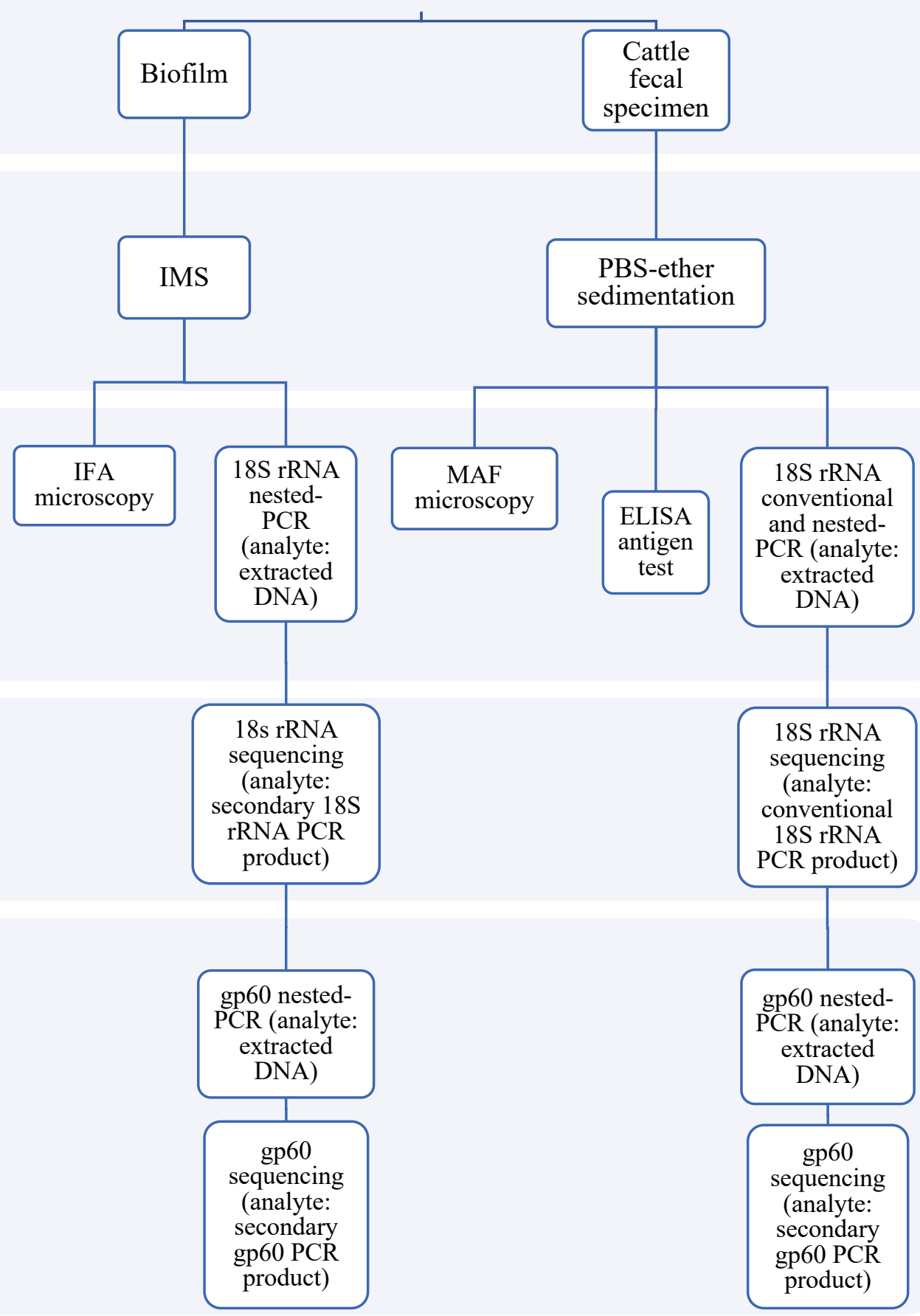

Figure 2.1.: Summary of experimental workflow. 


\subsection{General Description of Study Region}

In Jamaica, there are 14 parishes and 26 watersheds. The Black River watershed is named after the dark hue of its mainstream river bed, formed after years of decomposing vegetation. The watershed is predominately situated in St. Elizabeth — a rural parish located in the southwestern region of the island. St. Elizabeth is $1210.7 \mathrm{~km}^{2}$ and had a population of 150,993 in 2012 (Statistical Institute of Jamaica, 2017). The watershed also encompasses the southern regions of Westmorland, St. James and Trelawny, as well as the northwestern region of Manchester (Figure 2.2). The river itself measures $54.4 \mathrm{~km}$ and is maneuverable for about $40 \mathrm{~km}$. The river network is supported by many tributaries including YS, Broad, Grass and Horse Savannah, which forms the longest river system in Jamaica (Fiwi Roots, 2016).

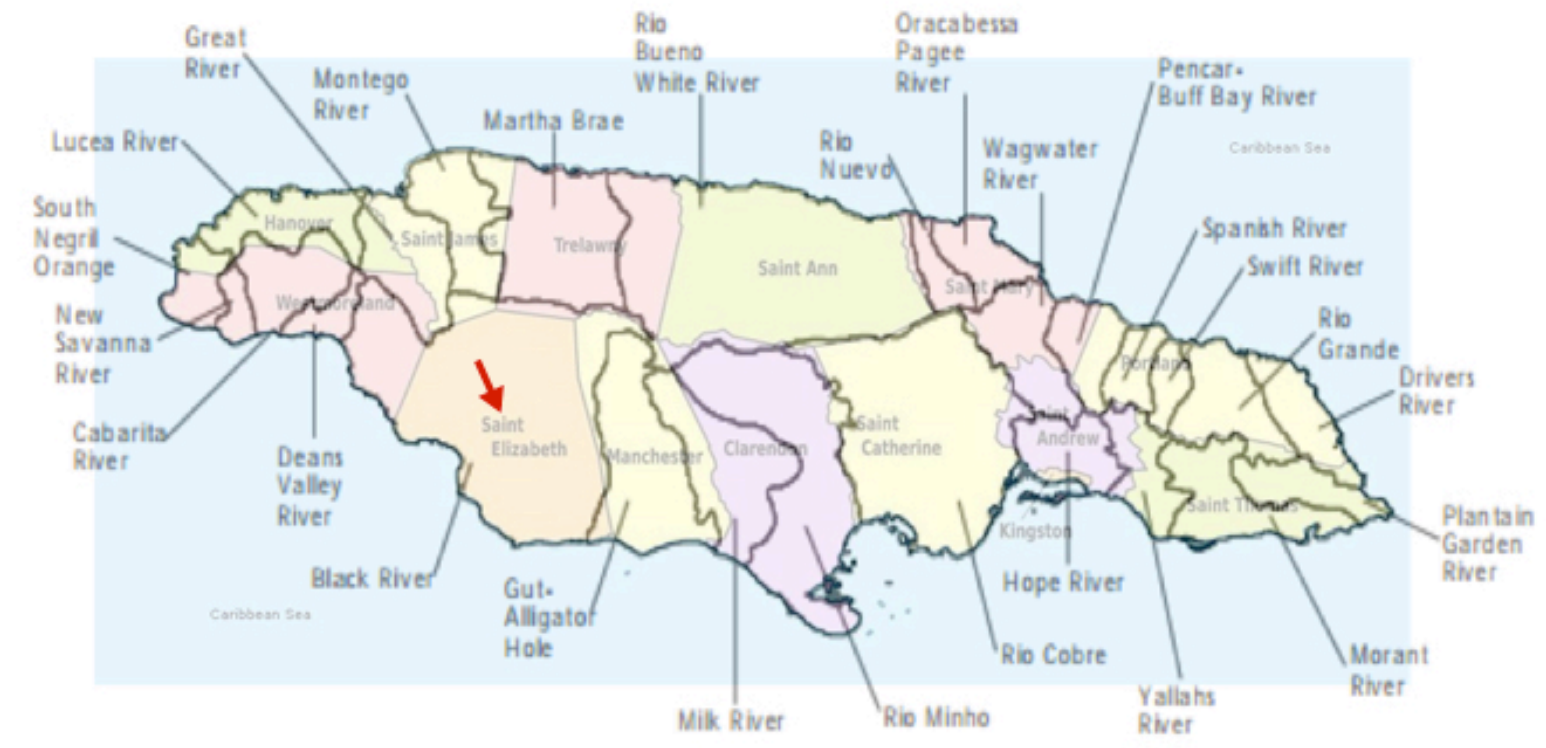

Figure 2.2. Fourteen parishes and twenty-six watersheds of Jamaica. Multi-colour basemap displays 14 parishes. This is overlaid by the solid black-lined map, which displays 26 watersheds. Adapted and modified from sources: Holness (2016) and NEPA (2003).

The river network is categorized into two main constituents; the Upper Morass and Lower 
Morass (Figure 2.3). The Upper Morass includes the highest point and middle region of the river, flowing through a swampy section, which serves as a settling basin and sink for nutrients (Caribbean Birding Trail, 2015; Mordab, Hong Yuan, \& Hu, 2013). The Lower Morass is Jamaica's largest wetland area — a declared wetland of importance under the United Nation's international treaty for the conservation and sustainable use of wetlands (Smith Warner International, 2005). From the Lower Morass, the river empties into the Caribbean Sea.

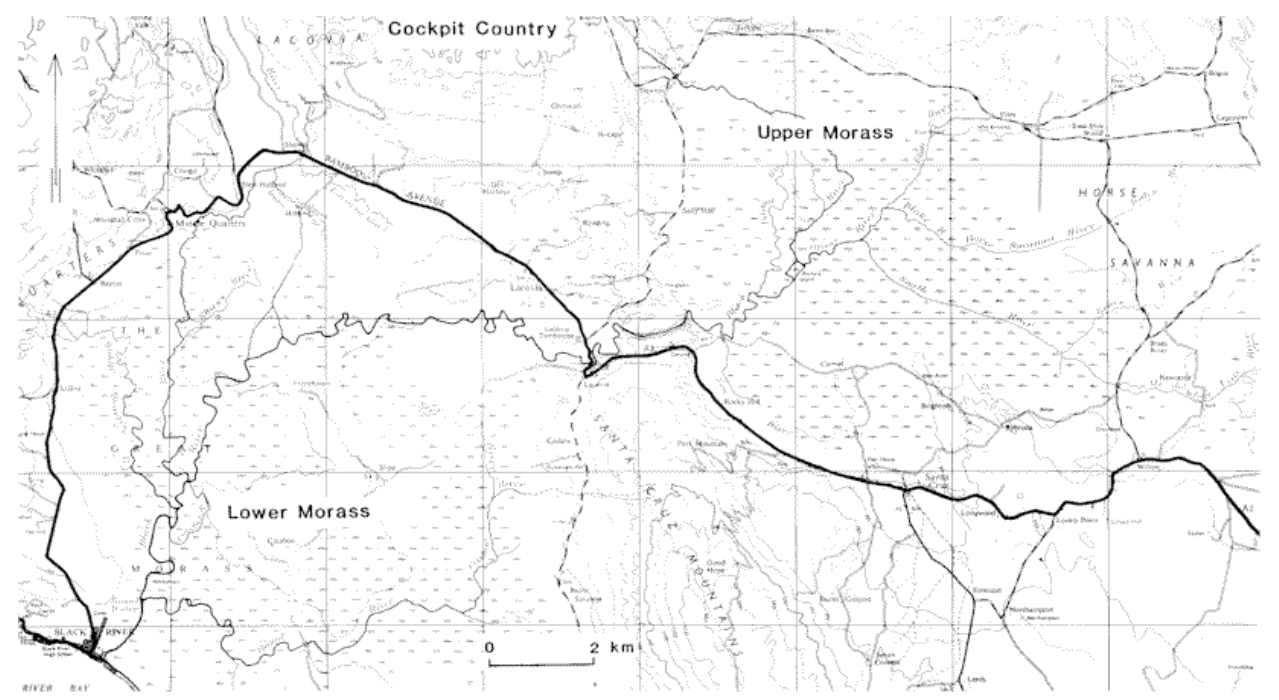

Figure 2.3.: Upper and Lower Morass of the Black River watershed. Source: Björk (2013).

The Black River receives influences from many human activities. Currently, the river is used for irrigation, fish and shrimp harvesting, and to take tourists on motor boat excursions. According to the Jamaican Protected Areas Trust Limited [JPAT] (2016), there is an urgent need for protection of the Black River banks due to increasing threats, including agricultural pollution. The river occupies the entire St. Elizabeth parish, which is known as the "bread basket" of the island, producing $22 \%$ of the nation's food (Jamaica Observer, 2014). Thus, agricultural production such as, livestock rearing, farming, irrigation and fertilization/cultivation of crops, collectively impact the water quality of the river. 


\section{Chapter 3: Materials and Methods}

\subsection{Pilot Study: Biofilm Development and Oocyst Recovery}

\subsubsection{Biofilm Sampler Design}

Three customized biofilm samplers were created using a metal frame measuring $21 \mathrm{~cm} \mathrm{x} 4 \mathrm{~cm} \mathrm{x}$ $14.8 \mathrm{~cm}$. Each frame had a front and back surface with permeable holes, allowing for water to infiltrate, while restricting the flow of large organisms, organic material and debris. At the base of the apparatus, there were four holes, which enabled the use of long pegs for nailing the sampler into the ground opposed to a conventional weight system as shown in section 1.8.3. (Figure 1.8d.). This feature secured the apparatus in an upright position despite high stream velocity. Inside the metal frame enclosed 8 slots for removable slides measuring $3.5 \mathrm{~cm} \mathrm{x} 0.24$ $\mathrm{cm} \times 14 \mathrm{~cm}$, which were made out of polycarbonate (plastic) substrate. Figure 3.1. provides photographs and illustrates the configuration of the biofilm sampler apparatus.

The rationale for using plastic slides rather than previous glass surfaces was to optimize biofilm development. Microorganisms attach more readily to hydrophobic nonpolar surfaces such as plastics compared to hydrophilic materials such as glass and metals (Bendinger, Rijnaarts, Altendorf, \& Zehnder, 1993; Fletcher \& Loeb, 1979; Pringle \& Fletcher, 1983). In addition, biofilms develop better on rough surfaces compared to smooth surfaces because they obstruct shear forces and produce a greater surface area for microbial colonisation (Charackalis, 1990). Last, the slides were positioned parallel to water flow to reduce collision and attachment of large organisms and debris. The positioning of the slides also facilitated the attachment of adhesive microorganisms forming EPS layered biofilms. Refer to, Figure G.I. in Appendix G for photographs of the biofilm sampler apparatus. 

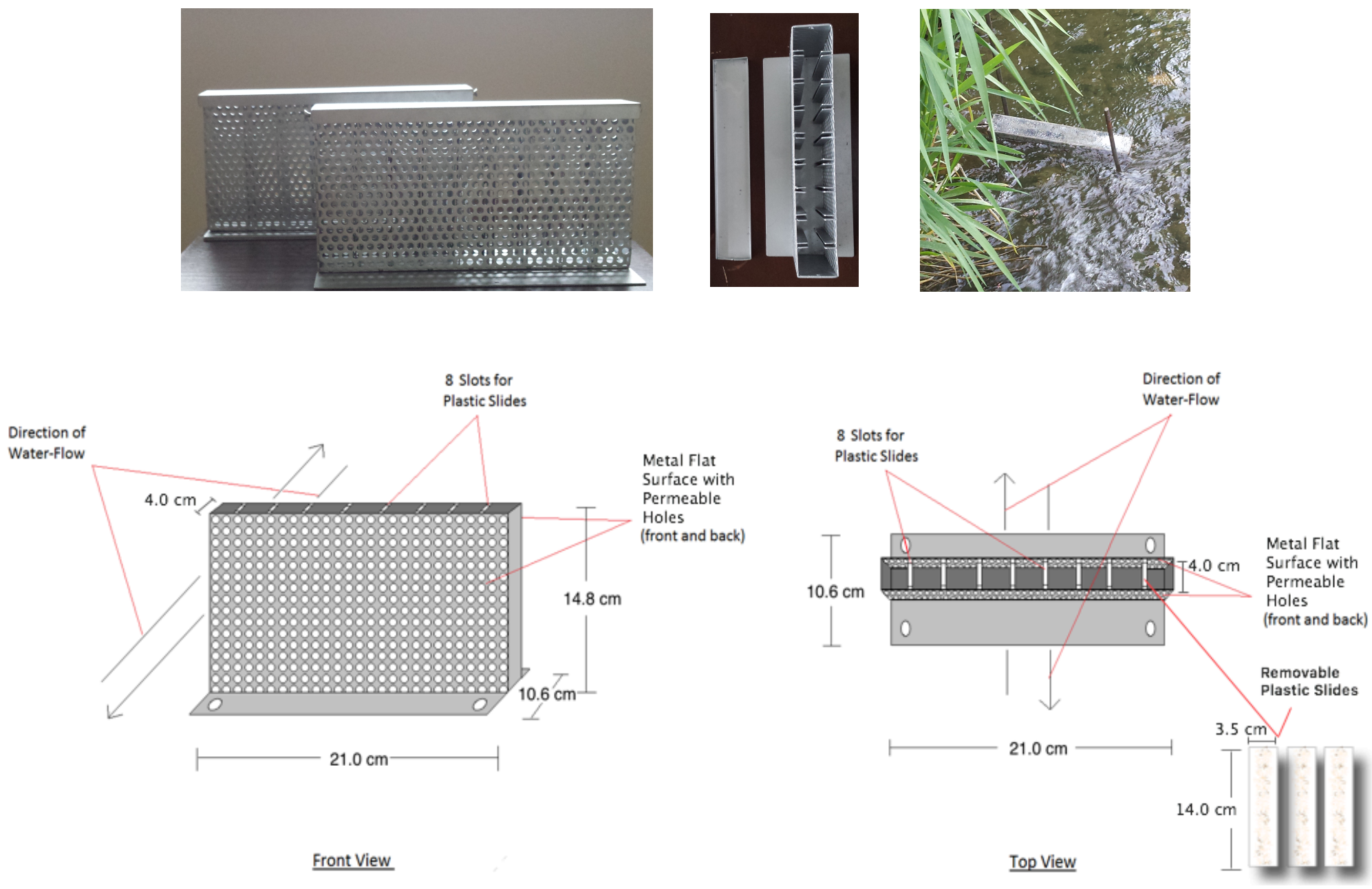

Figure 3.1.: Photographs and Diagram of the biofilm sampler apparatus with removable slides made out of polycarbonate material. Shown from the front and top view, illustrating the framework, dimensions and direction of water flow.

\subsubsection{Sample Collection}

During the spring of 2016, the biofilm sampler was inserted into shallow waters for 3-weeks, at approximately $50 \mathrm{~cm}$ depth in Alder Creek (Petersburg, Ontario). The specific timeframe was selected based on previous investigations, which found that mature biofilms require somewhere between 2 and 4-weeks to form complex structures, which generally plateau after the second 
week (Hunt \& Parry, 1998). Moreover, longer incubation periods would have resulted in sloughing; the sporadic detachment of large portions of the biofilm from its main structure (Kaplan, 2010).

A total of 4 plastic slides were introduced into the biofilm sampler, which served as growth surfaces for biofilms to develop on overtime. A glass reference-slide was also incubated alongside the polycarbonate substrate for comparative analysis. Prior to submergence in water, the slides were rinsed with $100 \%$ ethanol to remove any surface dirt or contamination. After 20 days, the slides were removed and put in a moist chamber, lined with a damp cloth to avoid dehydration. All samples were transported to the lab in an ice cooler, then stored in a refrigerator maintained at $4^{\circ} \mathrm{C}$ for analysis within 48 -hours.

\subsubsection{Fluorescent Staining and Confocal Z-stack Imaging of Biofilms}

One half of each biofilm-slide $(n=4)$ was stained with either Streptavidin Texas Red ${ }^{\mathrm{TM}}$ Conjugates or SYTO ${ }^{\text {TM }} 9$ or Green fluorescent stains SYTO ${ }^{\text {TM }} 9$ Green Fluorescent Nucleic Acid (Table A.III., Appendix A). First, a 1:100 dilution was made from the stains, which was loaded onto the biofilms and incubated for 45 minutes at $37^{\circ} \mathrm{C}$. Second, a Nikon D-Eclipse C1 si confocal microscope with EZ-C1 3.80 software was used to capture layered images of the biofilms from base to top using the z-stack icon at 40X objective. Each z-stack contained layered images of $2 \mu \mathrm{m}$ increments going down through the biofilms (Koh et al., 2013). Therefore, the number of z-stacks per biofilm varied according to thickness.

The red or green fluorescence of the biofilms was excited with $632 \mathrm{~nm}$ and $543 \mathrm{~nm}$ lasers, respectively. A total of 8 separate z-stack images were acquired for each biofilm slide from a 
single channel, which met criteria outline by Heydorn et al. (2000). Scan locations were randomly selected in the central region of the slides to exclude disrupted surfaces or excessive biofilm accumulation formed after removing the slides from the biofilm sampler slots. Last, the confocal images produced in EZ-C1 3.80 were saved as .ics files, then downloaded into the NISElements AR 3.10 imaging software. Three-dimensional images were created in ND Viewer (multidimensional image display) using the Volume Projection icon. Scale bar was added to all images.

\subsubsection{COMSTAT2 Analyses of Biofilms Thickness and Roughness}

Individual z-stack images of the biofilms were exported as .ics files from NIS-Elements AR 3.10 and downloaded into Microsoft Windows ImageJ Java 1.8 software (open source provided by the National Institute of Health [NIH]). These images were converted into greyscale and ome-tiff format. The COMSTAT2 function was selected and the biofilms were analyzed with Automatic Threshold (Otsu's method) and Connected Volume Filtering (CVF). The following parameters were assessed:

1. average thicknesses: the highest points $(\mu \mathrm{m})$ of all pixel-columns from each z-stack layer (excluding holes and cavities) from which the mean value is calculated (Vorregaard, 2008).

2. maximum average thickness: the highest pixel-column $(\mu \mathrm{m})$ from each $\mathrm{z}$-stack layer, from which the mean value is calculated (Vorregaard, 2008).

3. Dimensionless roughness coefficient $\left(\mathrm{R}_{\mathrm{a}}{ }^{*}\right)$ : average deviation from the average thickness (normalized and dimensionless). 
The following is the computing definition of dimensional roughness coefficient, according to Comstat 2 (2018):

$$
R_{a}^{*}=\frac{1}{N \cdot t_{\text {avg }}} \sum_{i=1}^{\text {slices }} \operatorname{spots}[i] \cdot\left(\text { thickness }[i]-t_{\text {avg }}\right)
$$

where $\mathrm{N}$ is the total number of spots found, spots [i] is the spots of slice $\mathrm{i}$, thickness [i] is the thickness defined by slice $\mathrm{i}$, and $\mathrm{t}_{\mathrm{avg}}$ is the average thickness found in the thickness distribution. Biofilms with low $\mathrm{R}_{\mathrm{a}}{ }^{*}$ were considered to be more homologous, thus smoother than biofilms with high $\mathrm{R}_{\mathrm{a}}{ }^{*}$.

\subsubsection{Calculating IMS-recovery Efficiency of Non-viable C. parvum Oocysts Seeded into}

\section{Biofilm Suspensions}

The remaining halves $(n=4)$ of the biofilms were suspended in water then seeded with non-viable C. parvum oocysts to calculate IMS-recovery efficiency. Roughly, $19.5 \mathrm{~cm}^{2}$ of intact biofilm (area of a standard microscope slide) was scraped from each slide using a sterile scalpel and suspended into a cuvette contain $12 \mathrm{ml}$ of filtered creek water. The water was filtered through a $0.2 \mu \mathrm{m}$ Pall Membrane Filter (Sigma-Aldrich, Inc., St. Louis, MO) as in Wolyniak et al. (2010), to maintain physical conditions for the natural microbial assemblages prior to IMS. These suspensions were transported to the lab, then seeded with $50 \mu \mathrm{l}$ of non-viable $C$. parvum oocysts from the Crypt-a-Glo ${ }^{\mathrm{TM}} \mathrm{G} / \mathrm{C}$ Direct Comprehensive Kit (Table A.I., Appendix A). This was approximately 10,000 oocysts per suspension. 
Turbidity was measured using an Orion ${ }^{\mathrm{TM}}$ AQ4500 Turbidimeter (Thermo Fisher Scientific Inc., Waltham, MA). Then, the mixtures were centrifuged at $1500 \mathrm{X} g$ for 10 minutes. The supernatants were poured off and the pellets were subsequently recovered via IMS, as described in section 3.2.2. The only difference was that the final IMS products were suspended into $200 \mu \mathrm{l}$ of $1.0 \mathrm{~N} \mathrm{NaOH}$.

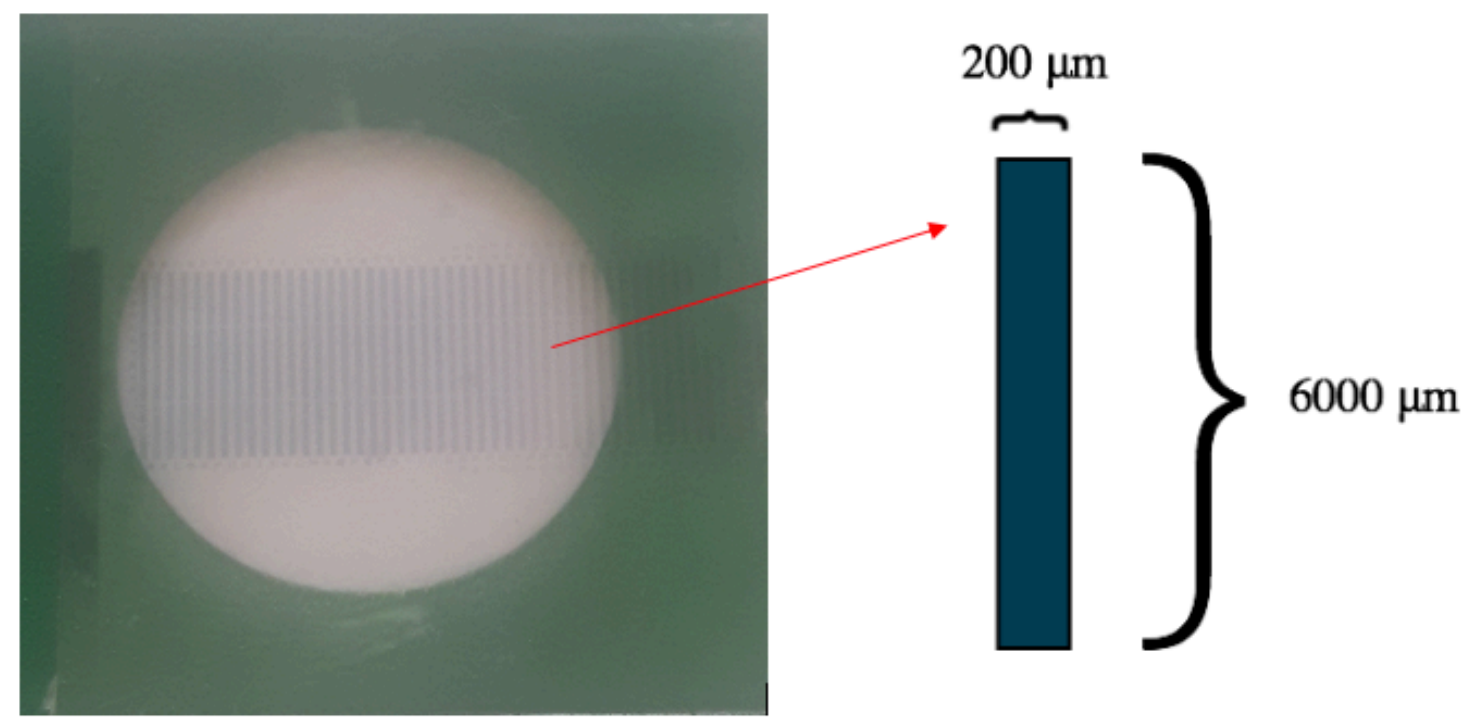

\section{Figure 3.2.: REF coverslip mounted onto a well slide to facilitate quantification of} Cryptosporidium oocysts. A total of 35 columns within the boundary of the well. Arrow pointing to diagram showing the dimension of a single column.

Fifty microlitres of IMS products were transferred to $15 \mathrm{~mm}$-SuperStick ${ }^{\mathrm{TM}}$ well slides $(n=4)$. The slides were dried and stained with Crypt-a-Glo ${ }^{\mathrm{TM}}$ as described in section 3.2.3. Relocatablegrid reference (REF) coverslips (Pang, 1996) were mounted onto the slides and the number of oocysts were quantified going down individual columns (Figure 3.2.). REF coverslips were implemented, rather than traditional clear coverslips in order to facilitate quantification. Previous studies have adapted REFs for asbestos fiber-counting by microscopists, which have 
demonstrated to improve accuracy, as well as provide opportunity for re-examination (Harper, Slaven, \& Pang, 2009; Pang \& Harper, 2008).

In total, there were 47 columns of examination according to the coverslip, 35 of which were fully within the boundary of the well. The dimension of each column is $6000 \mu \mathrm{m} \times 200 \mu \mathrm{m}$. By counting total $\mathrm{N}$ oocysts in $\mathrm{Z}$ number of columns, the concentration of oocysts was calculated as follows:

Conc. of oocysts $=\mathrm{NX} \frac{\text { Area of the well }}{\mathrm{Z} \text { x area of one column } \mathrm{x} \text { droplet volume } \mathrm{x} \text { dilution factor }}$

Note: $50 \mu$ fills the well completely

\subsection{IFA Detection of Cryptosporidium Oocysts from Biofilm Suspensions}

\subsubsection{Sample Collection}

Five biofilm samplers — each containing 8 plastic slides—were inserted into shallow waters in the Black River network. In July 2016, two samplers were inserted at Site A and Site B; while in February 2017, three samplers were inserted at Site C, Site D and Site E (Figure 3.3.). The samplers remained submerged under water for a period of 20-22 days. Once the samplers were recovered, the slides were carefully removed without disturbing the intact biofilms. A sterile scalpel was used to scrape off the biofilms from the slides (front and back) into a Whirl-pak ${ }^{\circledR}$ bag containing $40 \mathrm{ml}$ of filtered site water, as previously described in section 3.1.5. Each bag contained biofilms scraped from 4 slides. The samples were immediately transported to the 
University of the West Indies (UWI), Microbiology lab in an ice cooler and stored in a refrigerator maintained at $4{ }^{\circ} \mathrm{C}$.

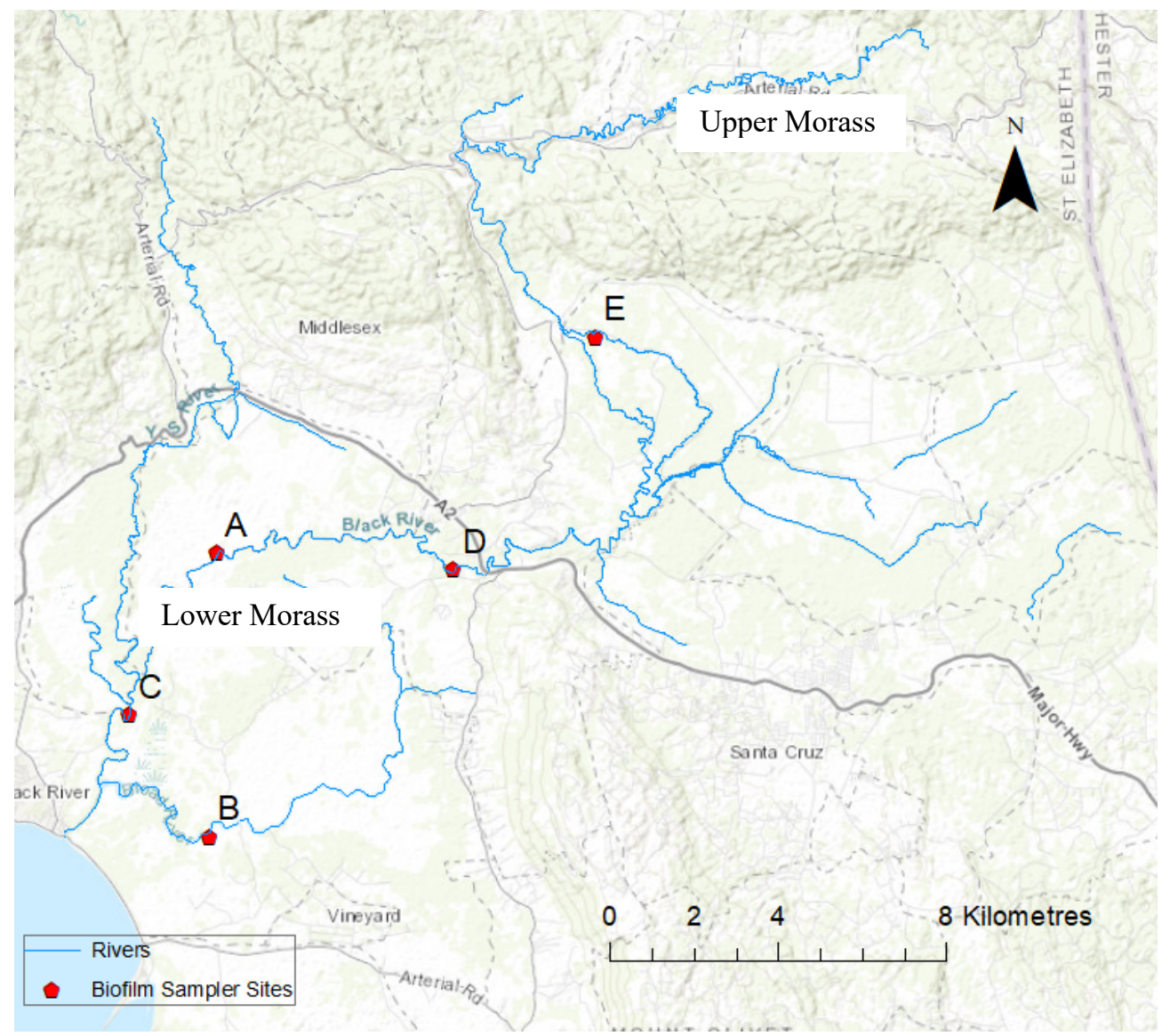

Figure 3.3. Biofilm sampler Sites A-E in the Black River Network, St. Elizabeth, Jamaica. Solid grey lines indicate major highways and roads; dashed lines indicate regional boundaries. Map created in ARCMap10 (ESRI Canada Limited).

\subsubsection{Centrifugation and IMS}

Centrifugation and IMS was performed within 48 hours of sample collection. The objective was to first concentrate biomass, then purify any oocysts contained within the biofilm suspensions. The Dynabeads ${ }^{\mathrm{TM}}$ anti-Cryptosporidium kit (Table A.I., Appendix A) was used to perform IMS 
as recommended by EPA Method 1623 (US EPA, 2005). The procedure uses magnetic beads that are coated with monoclonal antibodies designed to bind specifically to Cryptosporidium oocysts. All steps followed the manufacturer's protocol, except for minor adjustments discussed below.

First, $40 \mathrm{ml}$ of biofilm suspension was divided into 4 centrifuge tubes $(10 \mathrm{ml}$ ea.). The tubes were centrifuged at $1500 \mathrm{x}$ g for 10 minutes allowing for biomass to pellet, and the supernatant was carefully discarded. When necessary, the pellets were adjusted to $500 \mu$ lor less by splitting the pellet into halves using a disposable applicator stick, followed by re-centrifugation. Each pellet was added to a L-10 tube containing $2 \mathrm{ml}$ of SL ${ }^{\mathrm{TM}}$ Buffers. Subsequently, $100 \mu \mathrm{l}$ of Dynabeads ${ }^{\mathrm{TM}}$ was added to the mixture. A positive control consisting of approximately $1.0 \times 10^{3}$ of non-viable C. parvum oocysts was processed alongside the test samples.

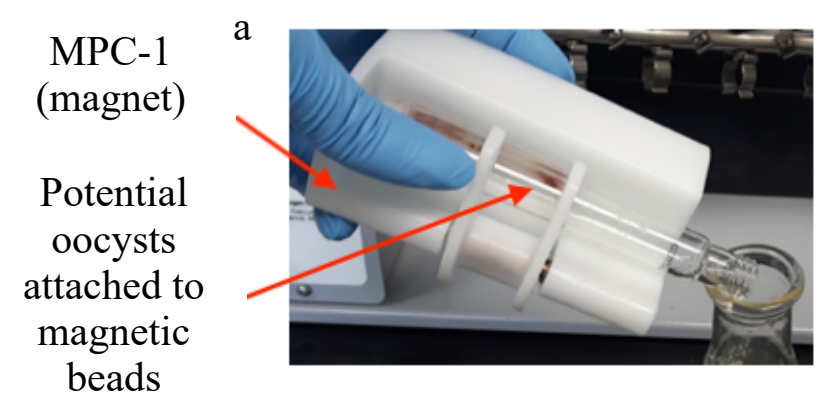

b

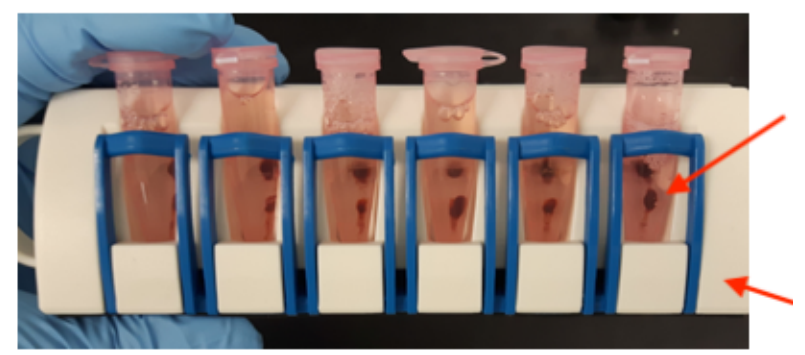

Potential oocysts attached to magnetic beads

MPC-S (magnet)

Figure 3.4.: MPC $^{\mathrm{TM}}$ magnets used for oocysts purification. L-10 tube attached to MPC-1 magnet (a) while supernatant is poured off. Microcentrifuge tubes in MPC-S magnet (b).

Here, a $\mathrm{pH}$ adjustment step was added to the protocol. The $\mathrm{pH}$ levels from all L-10 tubes were measured using an Orion ${ }^{\mathrm{TM}}$ 9810BN Micro pH Electrode (Thermo Fisher Scientific Inc., Waltham, MA). While the electrode was emerged in the solution for approximately 2 minutes, $\mathrm{pH}$ was adjusted to 7 by adding either $1.0 \mathrm{~N} \mathrm{NaOH}$ or $0.1 \mathrm{~N} \mathrm{HCl}$, depending on whether the 
solution was too acidic or basic. To avoid cross-contamination, the electrode was rinsed thoroughly with distilled water and dried between measurements, as recommended in the manufacturer manual. In a previous study, Khun, Rock and Oshima (2002) made these adjustments and determined that a neutral $\mathrm{pH}$ level improved oocysts recovery by $26.4 \%$ compared to unadjusted samples.

The L-10 tubes were inserted into a rotating mixer and incubated for 1-hour at 18-20 rpm. After incubation, the tubes were removed and attached to a Magnetic Particle Concentrator (MPC) ${ }_{-}^{\mathrm{TM}}$ 1. The beads were captured on the flat side of the tubes after rocking by hand for 2 minutes. While attached to the magnet, the caps were removed, and the supernatant was poured off (Figure 3.4a.). The magnet was detached, and the beads were resuspended into $400 \mu \mathrm{l}$ of $1 \mathrm{X}$ $\mathrm{SL}^{\mathrm{TM}}$ Buffer A, then transferred into corresponding $1.5 \mathrm{ml}$ microcentrifuge tubes. Each microcentrifuge tube was placed in a second magnet, the $\mathrm{MPC}^{\mathrm{TM}}-\mathrm{S}$ and rocked by hand for 1 minute (Figure 3.4b.). The supernatant was removed leaving potential oocysts bound to the beads, which were resuspended in $50 \mu \mathrm{l}$ of $1.0 \mathrm{~N} \mathrm{NaOH}$.

While the manufacturer's protocol recommends a final dissociation step, this was not performed. Normally, dissociation involves adding $5 \mu \mathrm{l}$ of $0.1 \mathrm{HCL}$ to the solution to detach oocysts from the magnetic beads. However, due to reports of poor dissociation, studies perform direct analyses to oocysts bound to the beads (Jiang et al., 2005; Xiao, Alderisio, \& Jiang, 2006). Thus, $5 \mu$ l of each product was transferred to a Spot-On ${ }^{\mathrm{TM}}$ well slide and air dried in preparation for IFA staining. The remaining products were stored in a freezer maintained at $-20{ }^{\circ} \mathrm{C}$ until DNA extraction.

\subsubsection{IFA Microscopy}


IFA microscopy was performed to examine biofilms using the Crypt-a-Glo ${ }^{\mathrm{TM}}$ Comprehensive Kit (Table A.I., Appendix A) as recommended by the EPA Method 1623 (US EPA, 2005). A total of 78 replicates were examined from 24 biofilm suspensions collected from 5 sites. First, the IMS products previously transferred to Spot-On ${ }^{\mathrm{TM}}$ slides were air-dried and fixed with methanol. Fifty microlitres of DAPI stain was added to each well and incubated at room temperature for 1 minute. The slides were rinsed with SureRinse ${ }^{\mathrm{TM}}$ wash buffer. Once dried, one drop of Crypt-a-Glo ${ }^{\mathrm{TM}}$ antibody reagent was added to each well. The slides were incubated for 45 minutes at $37^{\circ} \mathrm{C}$, then rinsed with the wash buffer. To reduce non-specific background fluorescence, one drop of BlockOut ${ }^{\mathrm{TM}}$ counterstain was added and incubated for 1 minute at room temperature. The slides were rinsed and a drop of No-Fade ${ }^{\mathrm{TM}}$ mounting medium was added along with coverslips.

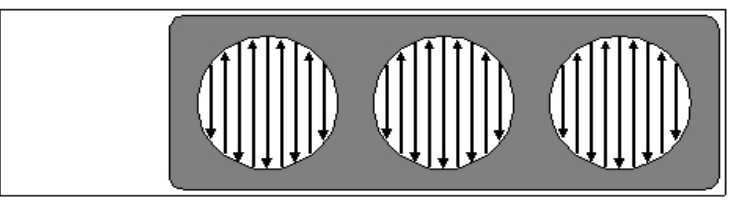

Figure 3.5.: Method 1623 examination pattern of well slide containing IFA-stained Cryptosporidium Oocysts. Protocol recommends that each well should be scanned in a systematic fashion (i.e., up-and-down pattern). Source: US EPA (2005).

A Nikon D-Eclipse C1 si confocal microscope with EZ-C1 3.80 software was used to identify Cryptosporidium oocysts. Green fluorescence was excited using a $543 \mathrm{~nm}$ laser. Figure 3.5 shows the examination pattern for all slides. The examination process started with $40 \mathrm{X}$ magnification objective to identify the presence of oocysts followed by confirmation with $100 \mathrm{X}$ oil immersion objective. The criteria for positive detection were: 1) brilliant apple green fluorescence; 2) round or oval shape; and 3) approximate diameter of 4-8 $\mu \mathrm{m}$, confirmed with scale bar. In addition, DIC objective was used to identify internal morphological characteristics, 
while a DAPI filter was used to identify sporozoites, which appeared a blue colour. The positive control described in section 3.2.2. was examined alongside the samples.

\subsection{MAF Detection of Cryptosporidium Oocysts from Cattle Feces}

\subsubsection{Sample Collection}

One hundred and nineteen cattle fecal samples were collected from 10 farms located in the Black River watershed during February, March and October 2017. Only 1 specimen was collected per animal. Figure 3.6. displays the geographic locations of each farm and the number of samples collected to scale. Specimens were transported to the microbiology laboratory in an ice cooler and stored in a refrigerator maintained at $-20^{\circ} \mathrm{C}$ until further processing.

Fifty-nine of the specimens derived from beef cattle and 60 were from dairy cattle. Qualitative data pertaining to age, sex, production type, and diarrheal symptoms were recorded at the time of sample collection for further interpretation of results. The age of cattle ranged between 0.1 and 11 years.

Selection criteria of farms included: small to medium family owned dairy or beef herds, ranging from 20 to 100 cows per farm. Also, each cow was either born or resided on the farms for at least 4 weeks. Additional information about drinking water sources(s), land rearing/flooring system, hygiene maintenance, and cow-calf separation was recorded at the time of sample collection and during follow up phone interviews, to obtain a general consensus of farm management. 


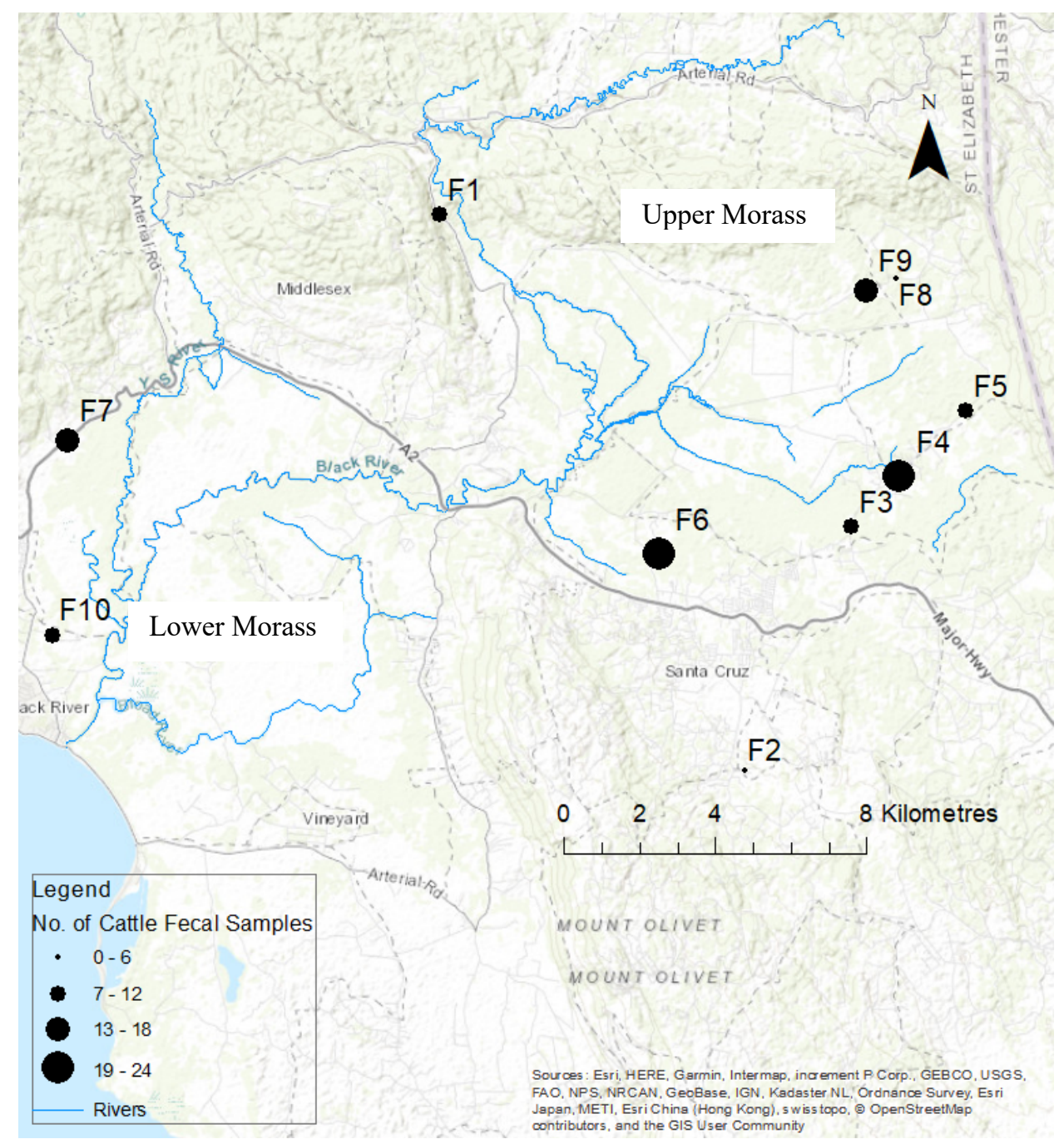

Figure 3.6.: Cattle farms F1-F10 and distribution of fecal specimens collected in the Black River watershed, St. Elizbeth, Jamaica. Solid grey lines indicate major highways and roads; dashed lines indicate regional boundaries. Map created in ARCMap10 (ESRI Canada Limited). A total of 60 and 59 specimens were collected from individual dairy and beef cows, respectively.

\subsubsection{Filtration and Phosphate Buffer Saline (PBS)-Ether Sedimentation}

One of the major difficulties in recovering Cryptosporidium oocysts from fecal specimens is attaining the required concentration for microscopy and PCR. In light of this challenge, filtration and PBS-ether sedimentation was used to concentrate oocysts from cattle feces. This procedure typically uses $10 \%$ formalin rather than PBS; however, formalin is a known inhibitor of PCR 
assays (Groelz et al., 2013). According to Dietrich et al. (2013), formalin causes random fragmentation of short DNA debris, which bind to the polymerase enzyme, thus interferes with the reaction. In addition, PBS is a better alternative because it is non-toxic, which prevents the rupturing and shrivelling of cells due to osmosis (Martin et al., 2006). Given these factors, several studies have adopted PBS-ether sedimentation prior to microscopic and PCR detection of Cryptosporidium oocysts (Freire-Santos et al. 2002; Gómez-Couso, Méndez-Hermida, \& AresMazás, 2006; Mendonça et al., 2007).

In the following research, PBS-ether sedimentation was performed on 119 cattle feces as per Waldman, Tzipori, \& Forsyth (1986) with minor adjustments. A positive control sample seeded with approximately $1.0 \times 10^{3}$ non-viable $C$. parvum oocysts was assessed alongside the samples. Table A.II. in Appendix A provides further details about all reagents used for sedimentation.

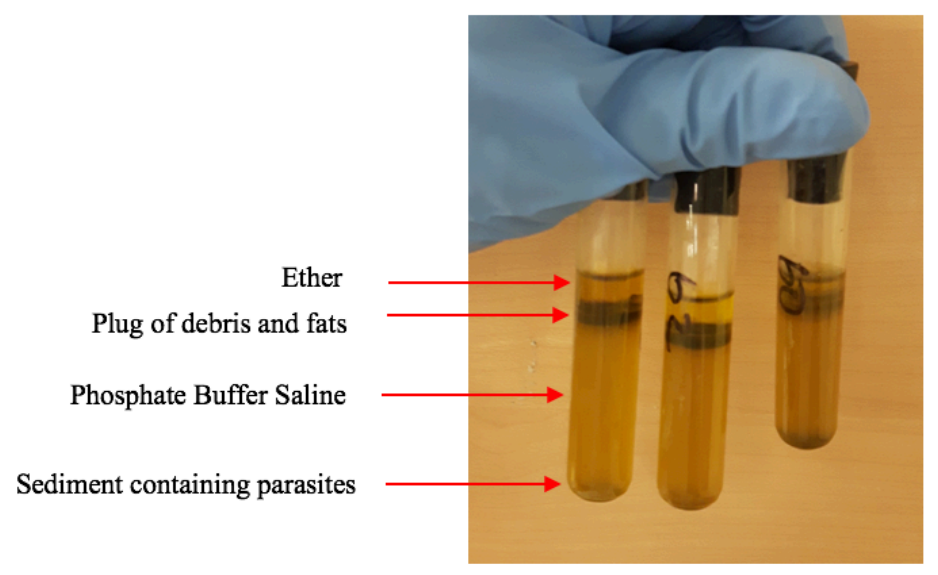

Figure 3.7.: PBS-ether sedimentation of cattle fecal specimen. Arrows pointing to four layers of separation post centrifugation.

First, 1-2 $\mathrm{g}$ of fecal specimen was transferred to a $10 \mathrm{ml}$ test tube containing $5 \mathrm{ml}$ of PBS. If the fecal specimen was liquid, approximately $1.5 \mathrm{ml}$ was transferred to the test tube. To remove larger particles, the specimen was filtered through a sieve made out of medical gauze. The 
resulting homogenate contained $7 \mathrm{ml}$ of fecal material (30\% [vol/vol] in PBS). Approximately, 2-3 $\mathrm{ml}$ of ether was added to the mixture. The mixture was centrifuged for 10 minutes at $500 \mathrm{x} g$. The resultant product was comprised of four layers, which included ether, a section of debris and fats, PBS and sediment containing potential parasites (Figure 3.7). A swab stick was carefully passed around the circumference of the tube to loosen the sheet of debris separating the two liquids. Then, the top three sections were discarded, permitting only the final sediment at the bottom. Last, $100 \mu 1$ of nuclease-free water was added to the sediment and $5 \mu 1$ of the suspension was transferred to a glass slide in preparation for MAF staining and microscopy. The remaining suspension of sediment was stored at $-20^{\circ} \mathrm{C}$ until DNA extraction.

\subsubsection{MAF Staining and Microscopy}

MAF staining and microscopy was performed to identify Cryptosporidium oocysts from cattle feces according to the CDC Modified Acid-Fast Staining Procedure (CDC, 2016b) with minor adjustments. The Ward's ${ }^{\circledR}$ Chemistry Acid-Fast Stain Kit was used for the reagents, but staining followed guidelines provided in CDC's protocol (Table A.II., Appendix A).

First, the microscope slides containing $5 \mu \mathrm{l}$ of sediment were air dried, then fixed with $99.8 \%$ methanol for 30 seconds. Fixation of the samples ensured that oocysts retained their morphological entity throughout the process (Ibrahim et al., 2016). Next, the slides were rinsed with distilled water and carbol fuchsin was added, then incubated at room temperature for 4 minutes. The slides were washed and decolorized with acid alcohol until they ran clear. Last, the slides were counterstained with methylene blue solution and incubated at room temperature for 2 minutes. The slides were then rinsed, dried and a coverslip was added. 
The examination process started with $40 \times$ magnification objective to identify the presence of Cryptosporidium oocysts followed by confirmation using $100 \times$ oil immersion objective. Scale bar was used to measure the diameter of oocysts. If a single oocyst appeared a pinkish-red colour with a diameter of approximately $4-8 \mu \mathrm{m}$, then the sample was considered positive. Two positive controls were used as reference to confirm detections: 1) positive control seeded into fecal specimen as described section 3.3.2.; and 2) C. parvum slide (VWR, Mississauga, Ontario).

\subsection{ELISA Detection of Cryptosporidium Antigens from Cattle Feces and Microplate Reader Interpretation}

Frozen cattle feces $(n=119)$ were thawed in preparation for ELISA using the CRYPTOSPORDIUM TEST (Table A.IV., Appendix A). This kit was used in previous studies, which detected Cryptosporidium antigens from cattle from Belgium (Geurden, Berkvens, Geldhof, Vercruysse, \& Claerebout, 2006a), India (Khan et al., 2010) and Zambia (Geurden et al., 2006b). The test uses monoclonal and rabbit polyclonal antibodies against Cryptosporidium oocysts, to form a complex with the parasites' antigens when present.

First, $400 \mu 1$ of Diluent was transferred to a $2 \mathrm{ml}$ microcentrifuge tube, then approximately $0.1 \mathrm{~g}$ (about the size of a small pea) of fecal specimen was added to the tube and thoroughly mixed by vortexing. If the fecal specimen was liquid, $100 \mathrm{ml}$ was added. Second, $100 \mu \mathrm{L}$ of Diluent was transferred to each test well of the Microassay Plate and a Pasteur pipette was used to transfer 1 drop of the fecal dilution to each well. The plate was sealed with a plastic adhesive sheet, which was incubated at room temperature for 1 hour. After incubation, the plate was inverted and knocked on paper towel to discard content from the wells. Each well was rinsed with 1X Wash 
Solution five times, until the plate appeared cleaned. The plate was inverted and knocked on paper towel to remove the liquid. One drop of Conjugate was added to each well. The plastic adhesive sheet was seal over the plate and incubated at room temperature for 30 minutes. The same wash procedure was repeated five times. Two drops of Substrate were added to each well and incubated at room temperature for 10 minutes. Last, 1 drop of Stop Solution was added to each well, which changed the complexes to a yellow colour.

The intensity of yellow was an indicator of Cryptosporidium antigens. This was quantified using a Thermo Labsystems Opsys MR Microplate reader (Thermo Fisher, Walthan, MA). The absorbance was measured $450 \mathrm{~nm}$ and readings $\geq 0.150$ were considered positive, according to the manufacturer's protocol.

\subsection{Statistical Analysis}

Standard deviation was calculated to indicate the extent of deviation of average biofilm thickness and roughness measurements, as described in section 3.1.4. Relative standard deviation (RSD) was calculated to determine whether standard deviation was small or large in comparison to the mean. This was applied to turbidity levels of each biofilm suspension, and subsequent $C$. parvum oocysts recovery efficiencies, as described in section 3.1.5.

$\% \mathrm{RSD}$ is defined as follows:

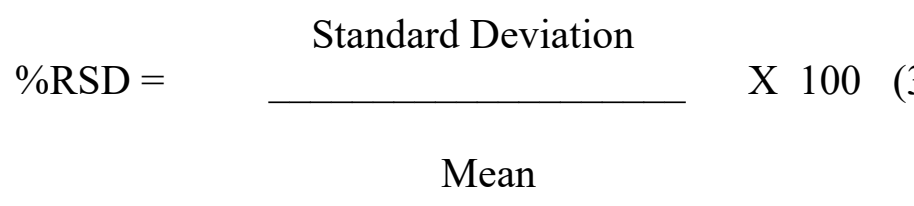


In terms of Cryptosporidium detection from cattle feces, the proportions of positive detections between dairy and beef animals were evaluated. Differences in proportions of positive detections were assessed using the Chi Square Test, p-values. In addition, diagnostic agreement between MAF microscopy and ELISA antigen test was statistically evaluated. PCR was not included in this analysis due to low detection sensitivity in cattle feces, relative to MAF and ELISA techniques. Diagnostic (inter-rater statistical) agreement between the two techniques was estimated using Cohen's kappa ( $k$ ) coefficient. Interpretation of the $k$ coefficient determined whether agreement was due to chance or systematic agreement (i.e., consistent patterns).

$k$ is defined as follows:

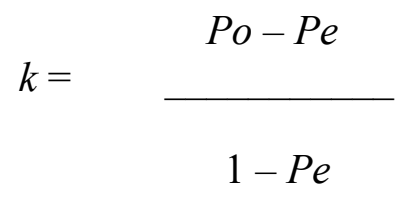

Where $P o$ is the observed agreement amoung raters, and $P e$ is the expected agreement (i.e., hypothesized probability of agreement by chance). The $k$ values were calculated by using the observed data to determine the probability of each observer randomly seeing each category. Interpretation of $k$ values was performed using the Byrt (1996) classification scheme, summarized in Table 3.1. 


\section{Table 3.1.: Summary of Proposed Kappa Classification Scheme}

\begin{tabular}{ll}
\hline Kappa Value & $\begin{array}{l}\text { Degree of } \\
\text { Agreement }\end{array}$ \\
\hline 0.00, or less & None \\
$0.01-0.20$ & Poor \\
$0.21-0.40$ & Slight \\
$0.41-0.60$ & Fair \\
$0.61-0.80$ & Good \\
$0.81-0.92$ & Very Good \\
$0.93-1.00$ & Excellent \\
\hline
\end{tabular}

Source: Byrt (1996)

\subsection{PCR Detection of Cryptosporidium}

\subsubsection{S rRNA Conventional and Nested-PCR}

PCR in conjunction with gel electrophoresis is the most widespread genotyping assay used for Cryptosporidium detection in research laboratories. Here, conventional PCR was performed for initial screening of Cryptosporidium from biofilms and cattle feces by amplifying fragments of the hypervariable region of the $18 \mathrm{~S}$ rRNA gene. This was achieved by using CPB-DIAG primers, which targeted the $435 \mathrm{bp}$. Samples confirmed positive by conventional PCR were subjected to nested-PCR using primers specified in Table 3.2. Non-specific bands observed from conventional PCR products — within \pm 50 bp of the 435 bp target — were also subjected to nestedPCR, in order to ensure that they were true negatives.

In general, nested-PCR was performed to improve sensitivity and specificity of detection by 
incorporating two sets of primers, which was suitable for detecting low number of oocysts typically found in environmental waters and fecal samples. Therefore, XIAO 1 and XIAO 2 nested primers were used to detect Cryptosporidium from cattle specimens, as described in Xiao et al. (1999). Whereas, KJL and CPB-DIAG nested primers were used to detect Cryptosporidium from biofilm suspensions, as described in Jellison et al. (2002).

Table 3.2.: Primers for $18 S$ rRNA Conventional and Nested-PCR

Forward and Reverse Primers and Sequences

CPB -DIAGF

AAG CTC GTA GTT GGA TTT CTG

CPB -DIAGR

TAA GGT GCT GAA GGA GTA AGG
PCR

Conventional $18 \mathrm{~S}$ rRNA
435

References 
The 18S rRNA was the most appropriate gene target selected for conventional and nested-PCR because Cryptosporidium spp. were detected from a diverse pool of environmental samples. Moreover, the 18S rRNA gene has the richest sequencing database available for Cryptosporidium compared to any other gene targets including: Cryptosporidium oocyst wall protein (COWP); Thrombospondin-related adhesive protein of Cryptosporidium-1 (TRAP-C1); and Heat shock protein 70 (hsp70) etc. (Xiao \& Ryan, 2008). Therefore, the high degree of sequence homology presented in the $18 \mathrm{~S}$ rRNA enabled the use of primers that could amplify a wide range of Cryptosporidium spp. isolated from environment sources (Kimbell, Miller, Chavez, \& Altman, 1999).

\subsubsection{Gp60 Nested-PCR}

Positive Cryptosporidium samples were further characterized using a nested-PCR assay, which amplified the variable region of the gp60 gene using primers specified in Table 3.3. Different combinations of these primers have been incorporated in previous studies to identify subtypes of C parvum and C. hominis from cattle feces (Alves et al., 2003; Hatalová, Valenčáková, \& Kalinová, 2017) and wastewater sources (Feng, Li, Duan, \& Xiao, 2009; Glaberman et al. 2002). Overall, gp60 characterization of Cryptosporidium was an important component, as it revealed the epidemiological relevance of positive detections. 
Table 3.3.: Primers for gp60 Nested-PCR

\begin{tabular}{|c|c|c|c|c|}
\hline $\begin{array}{l}\text { Forward and Reverse Primers and } \\
\text { Sequences }\end{array}$ & PCR & $\begin{array}{l}\text { Targeted } \\
\text { Gene }\end{array}$ & bp & References \\
\hline $\begin{array}{l}\text { Gp60F1 } \\
\text { ATG AGA TTG TCG CTC ATT ATC G } \\
\text { Gp60R1 } \\
\text { TTA CAA CAC GAA TAA GGC TGC }\end{array}$ & $\begin{array}{l}\text { Nested } \\
\text { (Outer) }\end{array}$ & gp60 & $\begin{array}{l}980- \\
1000\end{array}$ & $\begin{array}{l}\text { Strong, Gut, } \\
\text { and Nelson } \\
(2000)\end{array}$ \\
\hline $\begin{array}{l}\text { AL3532 } \\
\text { TCC GCT GTA TTC TCA GCC } \\
\text { AL3535 } \\
\text { GGA AGG AAC GAT GTA TCT }\end{array}$ & $\begin{array}{l}\text { Nested } \\
\text { (Inner) }\end{array}$ & gp60 & $\begin{array}{l}800- \\
850\end{array}$ & $\begin{array}{l}\text { Peng et al. } \\
\text { (2001) and } \\
\text { Alves et al. } \\
(2003)\end{array}$ \\
\hline
\end{tabular}

\subsubsection{Oocysts Rupture and DNA Extraction}

Oocyst rupture and DNA extraction was performed on the cattle feces and biofilm samples using the QIAamp® Fast DNA Stool Mini Kit and reagents listed in Table A.V. in Appendix A. Two positive controls were assessed alongside the samples. First, to rupture oocysts from the biofilm suspensions, $200 \mu \mathrm{l}$ of each packed pellet, previously isolated via IMS was transferred to a 1.5 $\mathrm{ml}$ microcentrifuge tube. Then, $50 \mu \mathrm{l}$ of $20 \%$ (w/v) suspension of Chelex ${ }^{\circledR} 100$ Resin was added to the mixture to reduce potential degradation of DNA. The samples were then subjected to seven cycles of freezing and thawing at $-70^{\circ} \mathrm{C}$ for 15 minutes and $70^{\circ} \mathrm{C}$ for 15 minutes. The same procedure was repeated for the cattle specimens, which were previously concentrated via PBS-ether sedimentation. The remaining extraction process was performed using the QIAamp® Fast DNA Stool Mini Kit as per the manufacturer's protocol with minor adjustments.

Briefly, the sample pellets were suspended in $1 \mathrm{ml}$ InhibitEX Buffer and incubated at $70^{\circ} \mathrm{C}$ for 5 minutes. The samples were centrifuged at 20,000 x g to pellet the particles. The supernatant was transferred to a new $1.5 \mathrm{ml}$ centrifuge tube containing $200 \mu \mathrm{l}$ Buffer $\mathrm{AL}$, and $15 \mu \mathrm{l}$ of proteinase 
$\mathrm{K}$ was added to the lysate. Next, the lysate was incubated at $70^{\circ} \mathrm{C}$ for 10 minutes and $200 \mu \mathrm{l}$ of $100 \%$ ethanol was added. The entire lysate was transferred to the QIAamp spin column and centrifuged at 20,000 x g for 1 minute. This process was repeated 2-3 times until the full lysate was filtered through the QIAamp spin column. Next, $200 \mu$ of Buffer AW1 was added to the QIAamp spin column, which was centrifuged at 20,000 x g for 1 minute. Subsequently, $200 \mu$ of Buffer AW2 was added to the lysate and centrifuged at 20,000 x g for 3 minutes. Last, $50 \mu 1$ of Buffer ATE was added to the QIAamp spin column, then centrifuged for 1 minute to elute the DNA. Notably, the final three Buffers added to the lysate was reduced from the original protocol to concentrate the Cryptosporidium DNA, as it was anticipated to be very low from environmental sources (Jiang et al., 2005). The extracted DNA samples were stored in a $-20^{\circ} \mathrm{C}$ freezer until PCR.

\subsubsection{Spectrophotometric Analysis}

Spectrophotometric analysis was performed to determine optimal primer dilution prior to genotyping assays. It was also used to evaluate the purity and concentration of DNA from purified PCR products. The NanoDrop ND1000 (Thermo Fisher, Walthan, MA) measured the solutions' absorbance spectrum between 220 and $330 \mathrm{~nm}$ to quantify concentration (ng/ $\mu \mathrm{l})$. Also, two absorbance ratios, $\mathrm{A}_{260} / \mathrm{A}_{280}$ and $\mathrm{A}_{260} / \mathrm{A}_{230}$ were recorded to evaluate purification.

First, dry primers were diluted in molecular grade water. NanoDrop ND1000 was erased using a blank cuvette. The solutions were inserted in the machine, and absorbance spectrum, ratios and concentration were recorded. Approximately, $40 \mathrm{ng} / \mu \mathrm{l}$ was considered to be optimal concentration of $5 \mu \mathrm{M}$ (working dilution) primers (Xiao \& Ryan, 2008). The primers were further diluted if they did not fall within this range. 
Second, the purity and concentration of DNA from PCR products were evaluated in the same manner as the primers. According to Themo Scientific (2009) $\mathrm{A}_{260} / \mathrm{A}_{280}$ and $\mathrm{A}_{260} / \mathrm{A}_{230}$ ratios indicate the presence of protein, phenol and/or other contaminants that absorb strongly at or near 280 and $230 \mathrm{~nm}$, respectively. Ratios between 1.8 and 2.0 for $\mathrm{A}_{260} / \mathrm{A}_{280}$ were considered pure. Similarly, ratios between 1.8 and 2.2 for $\mathrm{A}_{260} / \mathrm{A}_{230}$ were considered pure (Sambrook\& Russell, 2006; Thermo Scientific, 2009). Last, DNA of samples $<20 \mathrm{ng} / \mu \mathrm{l}$ were considered very low (Genomics Core Facility, n.d.), thus thermocycler conditions were adjusted for processing cattle DNA by increasing the number of cycles to 50 (refer to section 3.6.6). It is important to acknowledge that increasing the number of PCR cycles, may have generated more undesired mutations, resulting in primer dimers and a greater chance of false positives. However, this modification was necessary after multiple attempts to increase the concentration of extracted DNA. This included: modifying the DNA extraction procedure by decreasing the Buffer ATE volume for the final suspension step (section 3.6.3.); lowering the annealing temperature after running a temperature gradient test (section 3.6.6.); and testing alternative master mixes to determine most effective.

\subsubsection{Preparation of Master Mixes}

FastStart $^{\mathrm{TM}}$ Taq DNA Polymerase master mix, dNTPack (Table A.VI, Appendix A) was used to verify replicates of Cryptosporidium positive controls $(n=3)$, which were thereafter used in subsequent genotyping assays (refer to Figure D.III, Appendix D. for electrophoresis images). Positive controls derived from two sources: 1) human stool sample—clinically diagnosed positive for $C$. meleagridis in 2012. The use of human stool was approved by the UHWI/UWI/Faculty of Medical Sciences (FMS) Ethics Committee. 2) cattle fecal sample 
$\mathrm{C} 8 \mathrm{Cw}$, which was confirmed positive via MAF staining and ELISA as described in sections 3.3. and 3.4. Also, a negative control was included with every reaction.

Conventional and nested-PCRs, targeting the 18S rRNA, were performed with FastStart ${ }^{\mathrm{TM}}$ master mix in the Department of Microbiology laboratory at UWI. All reagents used to create the master mix were prepared under a biosafety cabinet, which was cleaned immediately before usage to avoid contamination. A final reaction volume of $50 \mu \mathrm{l}$ was prepared for each sample. The concentration and volume per reaction tube are provided in Table 3.4.

Table 3.4.: Preparation of Master Mix with FastStart ${ }^{\mathrm{TM}}$ Taq DNA Polymerase, dNTPack for 18S rRNA Conventional and Nested-PCR

\begin{tabular}{lcc}
\hline Reagents & Concentration & $\begin{array}{c}\text { Volume per } \\
\text { reaction tube }(\boldsymbol{\mu l})\end{array}$ \\
\hline PCR Buffer & $10 \mathrm{X}$ & 5 \\
Nuclease-free water & - & 33 \\
MgCl & $25 \mathrm{nM}$ & 3 \\
dNTPs & $20 \mathrm{mM}$ & 1 \\
Forward Primers & $10 \mu \mathrm{M}$ & 1 \\
Reverse Primers & $10 \mu \mathrm{M}$ & 1 \\
Taq Polymerase & $5 \mathrm{U} / \mu \mathrm{l}$ & 1 \\
Positive Control DNA/ & - & 5 \\
Primary PCR Product & & 50 \\
Final Volume & & \\
\hline
\end{tabular}

Note: the master mixes prepared for conventional and nested-PCR were identical 
AccuStart ${ }^{\mathrm{TM}}$ II PCR ToughMix master mix (Table A.VI, Appendix A) was used for $18 \mathrm{~S}$ rRNA conventional and nested-PCR detection of Cryptosporidium from biofilm and cattle fecal samples. The PCR assays were performed by The Hospital for Sick Children, The Centre for Applied Genomics (TCAG), Genetic Analysis Facility, Toronto, ON. A final reaction volume of $10 \mu 1$ was prepared for each sample. The concentration and volume per reaction tube are provided in Table 3.5 .

Table 3.5.: Preparation of Master Mix with AccuStart ${ }^{\mathrm{TM}}$ II PCR ToughMix for 18S rRNA Conventional and Nested-PCR

\begin{tabular}{lcc}
\hline Reagents & Concentration & $\begin{array}{c}\text { Volume per } \\
\text { reaction tube }(\mu \mathrm{l})\end{array}$ \\
\hline $\begin{array}{l}\text { AccuStart } \\
\text { ToughMix }\end{array}$ & $2 \mathrm{X}$ PCR & 5 \\
Nuclease-free water & - & 2 \\
Forward Primers & $5 \mu \mathrm{M}$ & 1 \\
$\begin{array}{l}\text { Reverse Primers } \\
\text { Extracted DNA/ Primary PCR }\end{array}$ & $5 \mu \mathrm{M}$ & 1 \\
Product & - & 1 \\
Final Volume & & 10 \\
\hline
\end{tabular}

Note: the master mixes prepared for conventional and nested-PCR were identical

GoTaq ${ }^{\circledR}$ DNA polymerase (Table A.VI, Appendix A) was used for gp60 nested-PCR detection of Cryptosporidium from positive biofilm and cattle fecal samples. This was performed prior to sequencing by the CDC, Division of Foodborne, Waterborne and Environmental Diseases, 
Atlanta, Georgia. A final reaction volume of $50 \mu \mathrm{l}$ was prepared for each sample. The concentration and volume per reaction tube are provided in Table 3.6.

Table 3.6.: Preparation of Master Mix with GoTaq ${ }^{\circledR}$ DNA polymerase for gp60 NestedPCR

\begin{tabular}{|c|c|c|c|}
\hline \multirow[t]{2}{*}{ Reagents } & \multirow[t]{2}{*}{ Concentration } & \multicolumn{2}{|c|}{ Volume per reaction tube $(\mu \mathrm{l})$} \\
\hline & & $\begin{array}{c}\text { Primary Master } \\
\text { Mix }\end{array}$ & $\begin{array}{l}\text { Secondary } \\
\text { Master Mix }\end{array}$ \\
\hline $\begin{array}{l}\text { PCR Buffer } 3 \text { (contain } \\
\mathrm{MgCl}_{2} \text { ) }\end{array}$ & $1 \mathrm{X}$ & 5 & 5 \\
\hline Nuclease-free water & - & 35.35 & 33.85 \\
\hline dNTPs & $1.25 \mathrm{mM}$ & 4 & 4 \\
\hline Forward Primers & $10 \mu \mathrm{M}$ & 1.25 & 2.5 \\
\hline Reverse Primers & $10 \mu \mathrm{M}$ & 1.25 & 2.5 \\
\hline BSA & $10 \mathrm{mg} / \mathrm{ml}$ & 2 & 0 \\
\hline Taq Polymerase 2 & $5 \mathrm{U} / \mu \mathrm{l}$ & 0.15 & 0.15 \\
\hline $\begin{array}{l}\text { Extracted DNA/Primary } \\
\text { PCR Product }\end{array}$ & - & 1 & 2 \\
\hline Final Volume & & 50 & 50 \\
\hline
\end{tabular}

\subsubsection{Temperature Gradient and Thermocycler Conditions}

The PCR assays described above have widespread applications for Cryptosporidium detection; however, there are reported drawbacks including reduced sensitivity and specificity from environmental samples, as mentioned in section 1.6.1. Given these limitations, optimization of 
18s rRNA primers was critical to perform prior to PCR. Thus, a temperature gradient was conducted, which determine band intensity at various annealing temperatures. These results helped to inform thermocycler conditions. For CPB-DIAG primers, the annealing temperatures ranged from 52.5 to $65^{\circ} \mathrm{C}$ and differed by $\pm 2.5^{\circ} \mathrm{C}$ increments. Similarly, XIAO nested primers ranged from 42.5 to $55^{\circ} \mathrm{C}$ and differed by $\pm 2.5^{\circ} \mathrm{C}$ increments.

PCR amplifications were performed with either the SimpliAmp ${ }^{\mathrm{TM}}$ Thermal Cycler or GeneAmp ${ }^{\circledR}$ PCR System 9700. For conventional PCR, the thermocycler conditions were as follows: an initial denaturation step at $95^{\circ} \mathrm{C}$ for 1 minute, then 35 cycles at $95^{\circ} \mathrm{C}$ for 15 seconds, $52.5^{\circ} \mathrm{C}$ for 15 seconds, and $72^{\circ} \mathrm{C}$ for 30 seconds with a final extension of $72^{\circ} \mathrm{C}$ for 7 minutes. For nested-PCR, the first round amplified the external PCR-products, which were used as templates for the nested reaction. The thermocycler conditions were relatively the same. The only difference was that the annealing temperature was increased to $55^{\circ} \mathrm{C}$, as described in Xiao et al. (2001). For all cattle samples, the number of PCR cycles were increased to 50 given the presence of low DNA. Finally, the thermocycler conditions for gp60 nested-PCR were as follows: an initial denaturation step at $94^{\circ} \mathrm{C}$ for 45 seconds, then 35 cycles at $94^{\circ} \mathrm{C}$ for 45 seconds, $52^{\circ} \mathrm{C}$ for 45 seconds, and $72^{\circ} \mathrm{C}$ for 60 seconds with a final extension of $72^{\circ} \mathrm{C}$ for 7 minutes. The primary and secondary rounds of PCR were identical.

\subsubsection{Gel Electrophoresis and Visualization}

PCR products were visualized by gel electrophoresis using reagents specified in Table A.VI., Appendix A. The positive and negative control PCR products were ran on 1.5\% agarose gel in TAE buffer. The gel was prepared by dissolving $1.2 \mathrm{~g}$ of Ultra-Pure ${ }^{\mathrm{TM}}$ agarose in $100 \mathrm{ml}$ of $0.5 \mathrm{X}$

TAE buffer in an Erlenmeyer flask. The solution was heated in a microwave to a full boiling 
point. Once cooled, $0.5 \mathrm{ml}$ of working stock Ultra-Pure ${ }^{\mathrm{TM}}$ ethidium bromide was added. The solution was thoroughly mixed and poured into a cast containing a comb row, which created the wells. The gel was allowed to harden for approximately 1 hour, then submerged into an electrophoresis tank containing $0.5 \mathrm{X}$ TAE buffer. Next, $3 \mu$ of DNA gel loading dye (6X) for each PCR reaction was spotted on parafilm and $20 \mu \mathrm{l}$ of the PCR products were added to the loading dye. The mixture was transferred to the wells and $10 \mu$ of PCR 50 - 2000 bp marker was added to the first well. The electrophoresis was carried out at $100 \mathrm{~V}$ for 1.5 hours. Bands of predicted size were visualized under UV light using a transmitter box. Gp60 PCR products were also ran on agarose gels in TAE buffer.

Biofilm and cattle PCR products were subjected to capillary electrophoresis using the QIAxcel DNA Fast Analysis Kit (Table A. IV, Appendix A). Ready-to-use gel cartridges, buffers, and a $50 \mathrm{bp}$ alignment maker was loaded into the instrument, along with microcentrifuge tubes containing PCR products. The process profile was selected, and the system was ran for 1.5 hours. Bands of the predicted size were visualized with the QIAxcel ScreenGel Software (Qiagen, Hilden, Germany). The DNA band intensities of all positive detections were ranked according to the following scheme: $++++=$ very strong,$+++=$ strong,$++=$ moderate,$+=$ weak intensity, and $-=$ no visualization (Adamska et al., 2012). This intensity scheme was referenced with positive control, $\mathrm{C} 8 \mathrm{Cw}$ shown in Figure 4.5c.

\subsubsection{Purification of Positive PCR products}

Purification of PCR products was performed partially at Ryerson University, and by The Hospital for Sick Children, genomic services. In both cases, the QIAquick ${ }^{\circledR}$ PCR Purification Kit (Table A.VII., Appendix A) was used as per the manufacturers protocol with minor 
adjustments. A 5:1 volume of Buffer PB was added to each PCR product and mixed. The product mixture was added to a Qiaquick spin column and centrifuged for 1 minute. Then, $1 \mathrm{ml}$ of Buffer PE was added to the spin column and centrifuged twice for 1 minute. Between each centrifugation, the flow-through solution was discarded. The Qiaquick spin column was placed in a new $1.5 \mathrm{ml}$ centrifuge tube, and $30 \mu \mathrm{l}$ of Buffer EB was added, followed by incubation for 1 minute and centrifugation for 1 minute. The DNA collected in the microcentrifuge tube was stored at $-20^{\circ} \mathrm{C}$ before submitting for sequencing.

\subsection{Gene Sequencing and Phylogenetic Analysis of Cryptosporidium spp. and Subtypes}

\subsubsection{Sequencing $18 S$ rRNA and gp60 PCR Products}

Sequencing of $18 \mathrm{~S}$ rRNA PCR products was carried out by The Hospital for Sick Children, genomic services with primers previously described in Table 3.2. Likewise, sequencing of gp60 PCR products was performed by the CDC with nested primers, AL3532/35 and gp60 described in Table 3.3. In both labs, the BigDye ${ }^{\circledR}$ Terminator v3.1 Cycle Sequencing Kit was used for the procedure (Table A.VIII., Appendix A). Purified amplicons were directly sequenced in the forward and reverse directions with either the Applied Biosystems 3730XL DNA Analyzer or Applied Biosystems 3130XL Genetic Analyzer.

\subsubsection{NCBI Basic Local Alignment Search Tool (BLAST) and Development of Phylogenetic Tree}

NCBI basic local alignment search tool (BLAST) was used to compare readable 18S rRNA sequences to the GenBank database. The online archive consisted of previously submitted 
Cryptosporidium sequences, most of which were from the same locus. Sequences were downloaded into ClustalX in FASTA format and aligned in "Multiple Alignment Mode", then manually trimmed. The output of aligned sequences produced gaps, which were included in the phylogenetic analysis, as it provides informative data about evolutionary paths/relationships (Evans, \& Warnow, n.d.).

The aligned sequences were downloaded into MEGA7 tree-building software and compared to previous submissions using the BLAST algorithm. If multiple clones from a single sample were sequenced with 95 to $100 \%$ identity, then the most homologous match was brought into MEGA7. Finally, the phylogenetic tree was constructing using the clustering algorithm, Neighbour-Joining, which evaluated the dissimilarity between aligned sequences (Moura, 2010). Various Cryptosporidium spp. were added to the tree, along with an outgroup organism, Eimeria tenella (accession number AF026388) that served as reference for determining evolutionary relationships within the ingroup. Last, a bootstrapping maximum likelihood method with 1000 replicates of original consensus alignments was used to access the accuracy of clustering results. Bootstrap values $50 \%$ or higher were included in the tree; however, the topology of the branch was considered "correct" only if the value of an interior branch was greater than 95\% (MEGA Software, 2011).

\subsection{ARC GIS Mapping}

ARC GIS mapping was conveyed to provide a visual representation of sampling allocations.

Two-dimensional maps were created using ARCMap10 (ESRI Canada Limited). The basemap of Jamaica pre-existed in the software and was used to allocate sampling sites displayed in Figures 3.3. and 3.6. To outline terrestrial boundary, an image of the Black River watershed (Figure 3.8.) 
was downloaded from Jamaica's National Environment and Planning Agency [NEPA] (2003).

The on-screen digitizing tool was used to convert the image into vector data by tracing its features onto the basemap. This tool was also used to convert the river network to a bright blue colour. A sampling location layer was added to the basemap by creating an input table in Microsoft Excel, which specified biofilm and cattle farm locations along with corresponding coordinates. The coordinates, in decimal degree latitude and longitude format, were retrieved at the same time of sample collection using the Maps Coordinate App. The table was exported as a .dbf file and the points were geocoded onto the basemap. A topography layer shown in Figure B.I in Appendix B was created using $20 \mathrm{~m}$ contour data, which was downloaded from DIVAGIS in the form of a Digital Elevation Model (DEM). Scale bar, compass and legend were added to the maps.

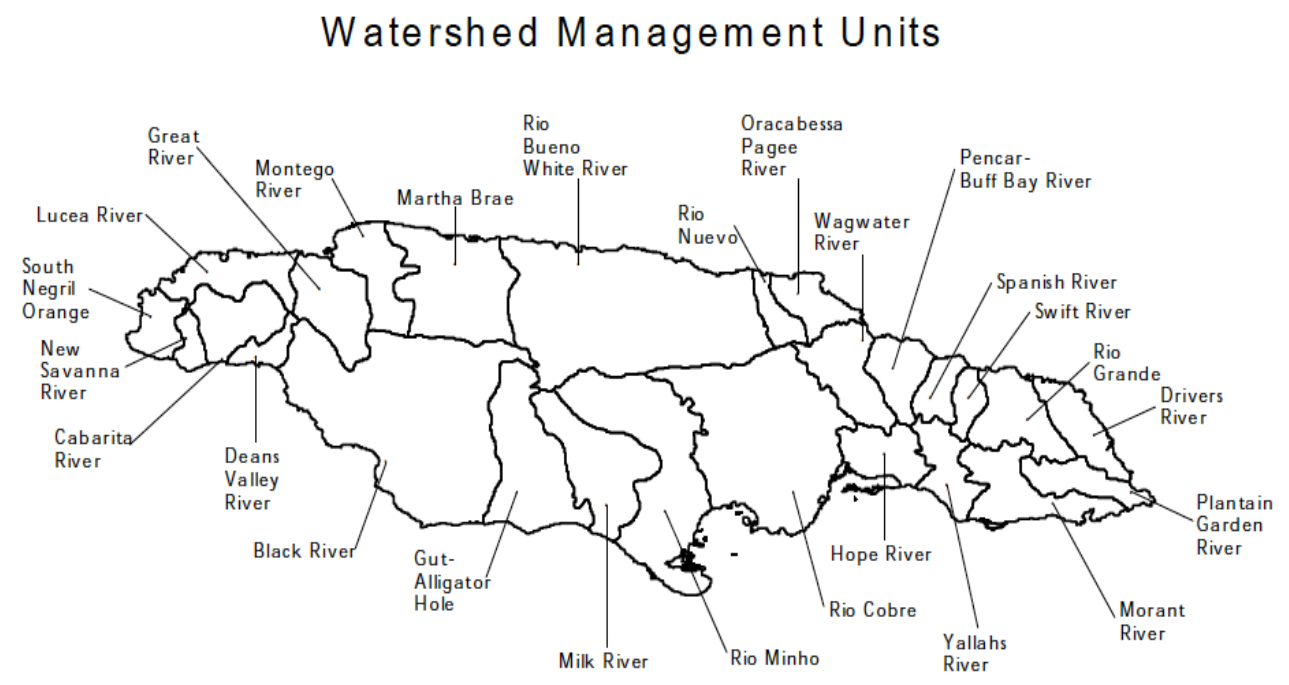

Figure 3.8.: Map used for outlining terrestrial boundary of the Black River watershed in ARCScene10 and ARCMap10. Source: NEPA (2003). 
Three-dimensional contour maps shown in Figure B.II. in Appendix B. was generated using ARCScene10 (ESRI Canada Limited). A raster image of elevation values was added to the scene layer. The same 20 m contour DEM used in ARCMap10 (ESRI Canada Limited) was brought into the software, and the base height was adjusted to reflect contour value. The sampling location layer was geocoded onto the scene layer. An image of the Black River network shown in Figure 2.3. was downloaded from Björk (2013). The on-screen digitizing tool was used to trace the river network onto the scene layer. Compass was added to the maps. 


\section{Chapter 4: Results}

\subsection{Method Validation and Performance of the Biofilm Sampler Technique}

\subsubsection{Biofilm Thickness and Roughness}

During the spring of 2016, a pilot study was performed in which biofilms were developed on 4 polycarbonate-slides and 1 glass reference-slide using a customized biofilm sampler. The purpose was to determine whether biofilms could reach an optimal level of maturation that could entrap and retain Cryptosporidium oocysts. After 3-weeks, a thin strip of biofilm formation was observed on all slides (Figure 4.1.). Three-dimensional, confocal images of biofilm segments were captured $(n=32)$ from half of each polycarbonate-slide and the entire glass reference-slide (approximate area of $19.5 \mathrm{~cm}^{2}$ from both substrate). The images were subsequently analyzed via COMSTAT2 to determine biofilm thickness and roughness. Findings are provided in Table 4.1., with complete data available in Table C.V., Appendix C.

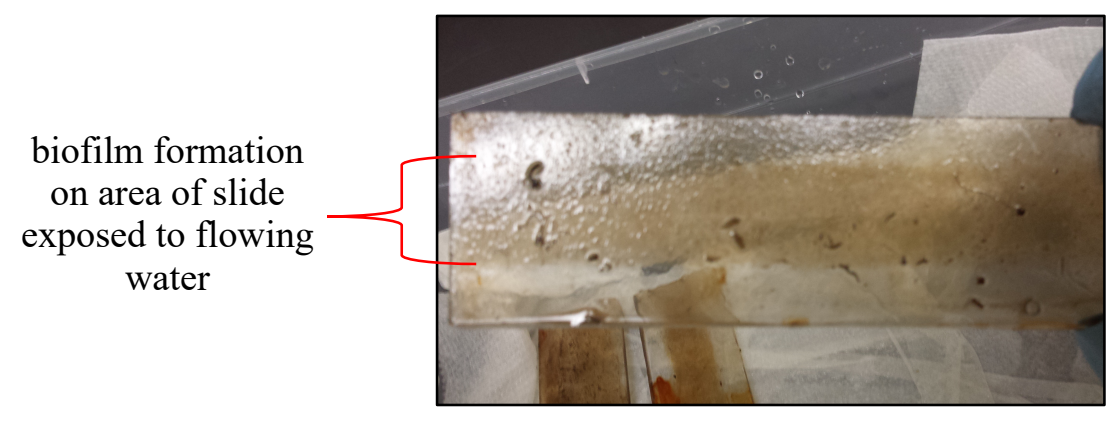

Figure 4.1.: Thin layer of biofilm formation on polycarbonate slides after 3-weeks submerged under water in biofilm sampler.

Average biofilm thickness measured from the polycarbonate-slides ranged from 48.33 to 82.53 $\mu \mathrm{m}$. While maximum average thickness was 95.63 to $115.13 \mu \mathrm{m}$. In contrast, biofilms developed on the glass substrate had an average thickness of $22.85 \mu \mathrm{m}$ and a maximum average thickness 
of $29.21 \mu \mathrm{m}$. Furthermore, microbial colonization was visibly thicker on the polycarbonate substrate compared to the glass reference-slide, as shown in Figure 4.3c. and $4.3 \mathrm{~d}$.

With regards to biofilm roughness, mean $\mathrm{R}_{\mathrm{a}}{ }^{*}$ of the polycarbonate-slides were between 1.63 and 1.78 , indicating that biofilms were variable in terms of thicknesses. These observations are reflective of biofilm patchiness or heterogenous formation (Figure H, Appendix $\mathrm{H}$ ). In contrast, mean $\mathrm{R}_{\mathrm{a}} *$ measured from the glass reference-slide was 0.49 , which indicates that biofilm formation was evenly distributed.

Table 4.1.: COMSTAT2 Analyses of Biofilm Thickness and Roughness Measured from Polycarbonate-Slides and a Glass Reference-Slide

\begin{tabular}{|c|c|c|c|}
\hline Biofilm ID & $\begin{array}{l}\text { Average Thickness } \\
(\mu \mathrm{m})^{\mathrm{a}}\end{array}$ & $\begin{array}{l}\text { Maximum } \\
\text { Thickness }(\mu \mathrm{m})^{\mathrm{a}}\end{array}$ & $\begin{array}{l}\text { Roughness } \\
\text { Coefficient }^{\mathrm{a}}\end{array}$ \\
\hline Bply $1^{\text {b }}$ & $48.33 \pm 29.41$ & $96.88 \pm 27.08$ & $1.76 \pm 0.21$ \\
\hline Bplyt $2^{b}$ & $52.78 \pm 27.47$ & $95.63 \pm 24.41$ & $1.73 \pm 0.26$ \\
\hline Bply3 ${ }^{b}$ & $82.53 \pm 17.83$ & $115.13 \pm 14.89$ & $1.78 \pm 0.25$ \\
\hline Bply $4^{\mathrm{b}}$ & $60.47 \pm 32.55$ & $100.13 \pm 17.88$ & $1.63 \pm 0.20$ \\
\hline BglsREF $^{c}$ & $22.85 \pm 4.54$ & $29.21 \pm 6.81$ & $0.49 \pm 0.27$ \\
\hline
\end{tabular}

${ }^{\text {a }}$ Mean \pm S.D. $(n=8)$

${ }^{\mathrm{b}}$ polycarbonate substrate

${ }^{\mathrm{c}}$ glass substrate

\subsubsection{Recovery Efficiency of C. parvum Oocysts Seeded into Biofilms}

The remaining biofilm-halves $(n=4)$ were suspended in water, then seeded with $C$. parvum 
oocysts. Recovery efficiency was then determined by IMS-IFA microscopy, using REF coverslips to facilitate quantification (Figure 4.2.). As shown in Table 4.2., mean recovery ranged between 23 and $68 \%$, with a seed dose of $2422 \pm 138$ oocysts and a mean turbidity of $548.1 \pm 25.95$ NTU. Complete data available in Table C.VI. of Appendix C. Therefore, oocysts recoveries were variable even though the seed dose and turbidity levels were fairly consistent, and the biofilms were developed simultaneously in a single biofilm sampler.

Counted oocysts within boundary of column

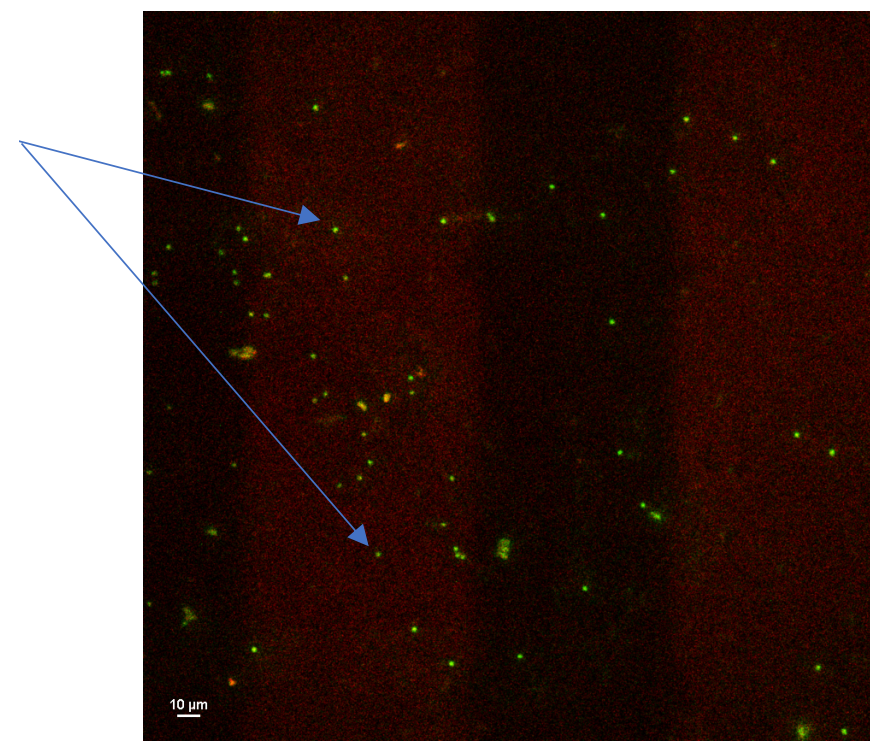

Gap between REF coverslip and well-slide

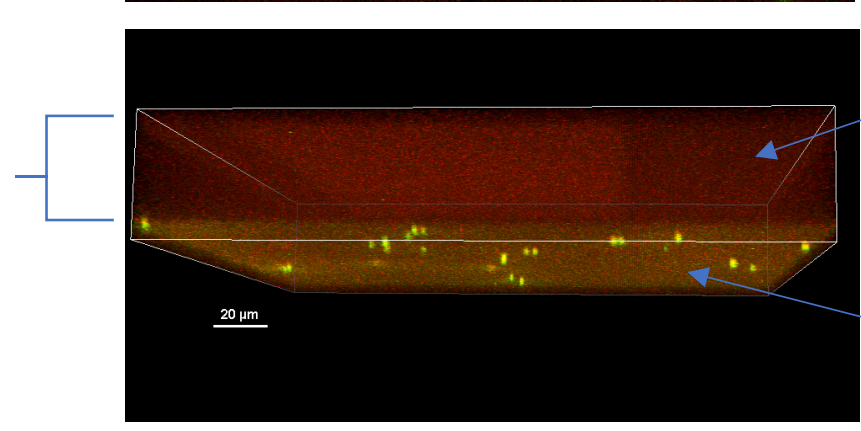

REF coverslip Well-slide containing oocysts

Figure 4.2.: Quantification of $C$. parvum oocysts from biofilm using IMS-IFA and REF coverslips displayed in 2D and 3D. Technique determined recovery efficiency of oocysts from biofilm suspensions. Number of oocysts (green) were counted by scanning up and down individual columns (red) within boundary of the well-slide. Magnification 40X. 
Table 4.2.: IMS Recovery of $C$. parvum Oocysts from Biofilm Suspensions

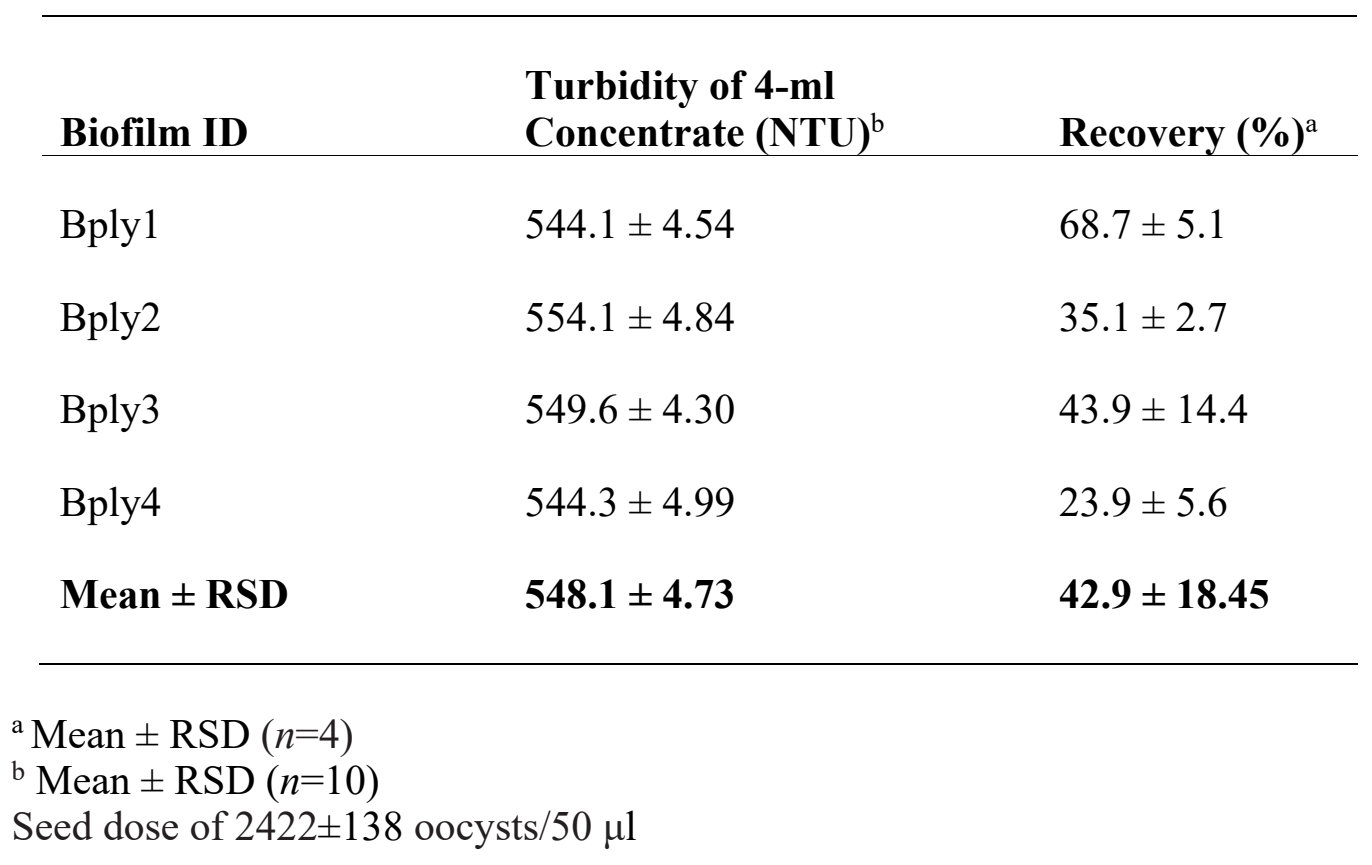

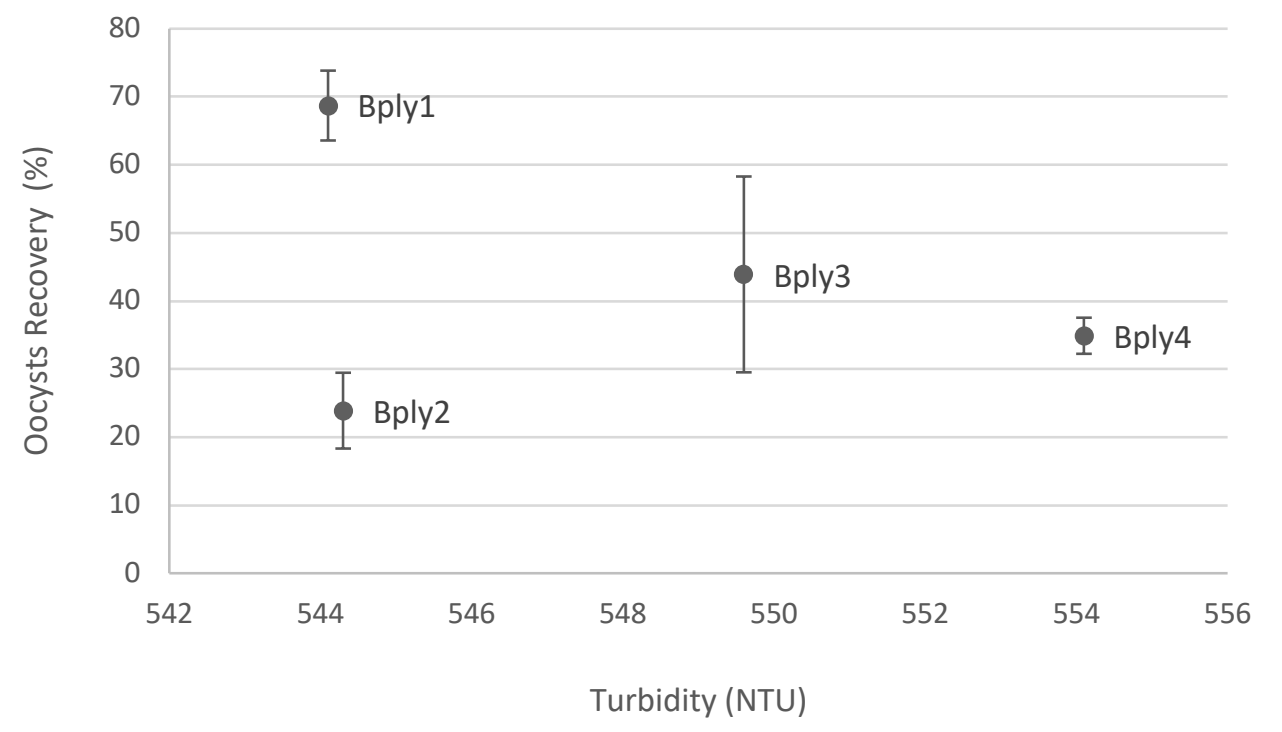

Figure 4.3.: Relationship between turbidity and recovery efficiency of Cryptosporidium oocysts seeded into environmental biofilms. Error bars indicate the S.D. between microscopeslide replicates $(n=4)$ for each biofilm suspension. 
Furthermore, the S.Ds. of oocysts recovery from biofilm suspensions, Bply1, Bply2, Bply3 and Bply4 were 5.1, 2.7, 14.4 and 5.6\%, respectively. Therefore, with the exception of Bply3, there were no significant differences between replicated microscope slides that were derived from a single biofilm (Figure 4.3.). Variability of oocyst recovery was dependent on the uniqueness of biofilm composition, rather than inconsistencies between slide replicates.

\subsection{Optimization of PCR Assays}

\subsubsection{Spectrophotometric Analysis of Primers and Purified DNA}

Spectrophotometry was performed to evaluate the concentration and purity of primers and purified DNA from selected biofilm and cattle samples. Original NanoDrop readings are available in Table C.VII., Appendix C.

Primer concentration and absorbance ratios were determined after appropriate dilutions were made from $100 \mu \mathrm{M}$ stock primers. As shown in Table 4.3., concentration of the final working dilutions ranged between 28 and $42 \mathrm{ng} / \mu \mathrm{l}$, which is consistent with $\sim 40 \mathrm{ng} / \mu \mathrm{l}$, as recommended in Fayer and Xiao (2008).

DNA concentration was determined from selected biofilm and cattle samples (Table 4.4.).

Surprisingly, biofilm samples C2Bio and D3Bio had a DNA concentration of 319.11 and 267.13 $\mathrm{ng} / \mu 1$, respectively. This is significantly higher than cattle sample $\mathrm{C} 8 \mathrm{Cw}$, which had a concentration of $10.57 \mathrm{ng} / \mu \mathrm{l}$. Figure 4.4. shows the absorbance spectrum, illustrating the vast difference between Cryptosporidium DNA extracted from the two sample types. 
Table 4.3.: Concentration and Purity of Primers

\begin{tabular}{|c|c|c|c|c|c|c|}
\hline \multirow[b]{2}{*}{ Primer } & \multicolumn{3}{|c|}{$100 \mu \mathrm{M}$ Stock Primers } & \multicolumn{3}{|c|}{$5 \mu \mathrm{M}$ Working Dilution Primers } \\
\hline & $\begin{array}{l}\text { Conc. } \\
(\mathrm{ng} / \mu \mathrm{l})\end{array}$ & $\begin{array}{l}\mathbf{A}_{260} / \mathbf{A}_{280} \\
\text { ratio }\end{array}$ & $\begin{array}{l}\mathbf{A}_{260} / \mathbf{A}_{230} \\
\text { ratio }\end{array}$ & $\begin{array}{l}\text { Conc. } \\
(\mathrm{ng} / \mu \mathrm{l})\end{array}$ & $\begin{array}{l}\mathbf{A}_{260} / \mathbf{A}_{280} \\
\text { ratio }\end{array}$ & $\begin{array}{l}\mathbf{A}_{260} / \mathbf{A}_{230} \\
\text { ratio }\end{array}$ \\
\hline CPB-DIAG Forward & 642.31 & 1.53 & 1.41 & 35.67 & 1.52 & 2.63 \\
\hline CPB-DIAG Reverse & 1125.65 & 2.06 & 2.71 & 42.12 & 1.54 & 2.14 \\
\hline KLJ Forward & 718.15 & 1.53 & 2.31 & 37.89 & 1.51 & 2.19 \\
\hline KLJ Reverse & 639.58 & 1.53 & 2.22 & 36.54 & 1.19 & 2.17 \\
\hline XIAO 1 Forward & 552.59 & 1.84 & 2.39 & 28.74 & 1.62 & 2.50 \\
\hline XIAO 1 Reverse & 596.40 & 1.70 & 2.10 & 30.05 & 1.49 & 2.22 \\
\hline XIAO 2 Forward & 736.67 & 2.06 & 2.93 & 40.76 & 1.94 & 2.88 \\
\hline XIAO 2 Reverse & 630.25 & 2.18 & 2.30 & 34.18 & 1.91 & 2.32 \\
\hline
\end{tabular}

Table 4.4.: Concentration and Purity of Selected DNA

\begin{tabular}{llll}
\hline Sample ID & Concentration $(\mathbf{n g} / \boldsymbol{\mu l})$ & $\mathbf{2 6 0 / 2 8 0}$ ratio & $\mathbf{2 6 0 / 2 3 0}$ ratio \\
\hline $\mathrm{C} 8 \mathrm{Cw}^{\mathrm{a}}$ & 10.57 & 2.65 & 0.07 \\
$\mathrm{C} 17 \mathrm{Cw}{ }^{\mathrm{b}}$ & 0.63 & 0.63 & 0.69 \\
$\mathrm{D} 3 \mathrm{Bio}^{\mathrm{c}}$ & 267.13 & 1.90 & 2.24 \\
$\mathrm{C} 2 \mathrm{Bio}^{\mathrm{c}}$ & 319.11 & 1.89 & 2.27 \\
$\mathrm{DNA} \mathrm{ctrl}$ & & 1.92 & 1.77 \\
$\mathrm{NFW}^{\mathrm{e}}$ & 90.32 & -1.67 & 2.67 \\
\hline
\end{tabular}

${ }^{a}$ DNA extract from Cryptosporidium-positive cattle feces

${ }^{b}$ DNA extract from Cryptosporidium-negative cattle feces

${ }^{c}$ DNA extract from Cryptosporidium-positive biofilms

d Positive Control DNA

${ }^{\mathrm{e}}$ Negative Control DN 
With regards to purity, the $\mathrm{A}_{260} / \mathrm{A}_{280}$ and $\mathrm{A}_{260} / \mathrm{A}_{230}$ ratios of sample $\mathrm{C} 8 \mathrm{CW}$ were 2.65 and 0.07 , respectively. These values do not fall within the acceptable "pure" range (between 1.8 and 2.2), as previously described in section 3.5.4. Therefore, excessive contaminants were present in sample $\mathrm{C} 8 \mathrm{Cw}$, which were anticipated to be present in the remaining cattle samples. In contrast, the $\mathrm{A}_{260} / \mathrm{A}_{280}$ and $\mathrm{A}_{260} / \mathrm{A}_{230}$ ratios of samples C2Bio and D2Bio were 2.27 and 2.24, respectively; thus, DNA from biofilms were very pure. Overall, these results suggest that Cryptosporidium DNA purified from the biofilm samples were significantly more concentrated and contained less PCR inhibitors compared to the cattle feces.

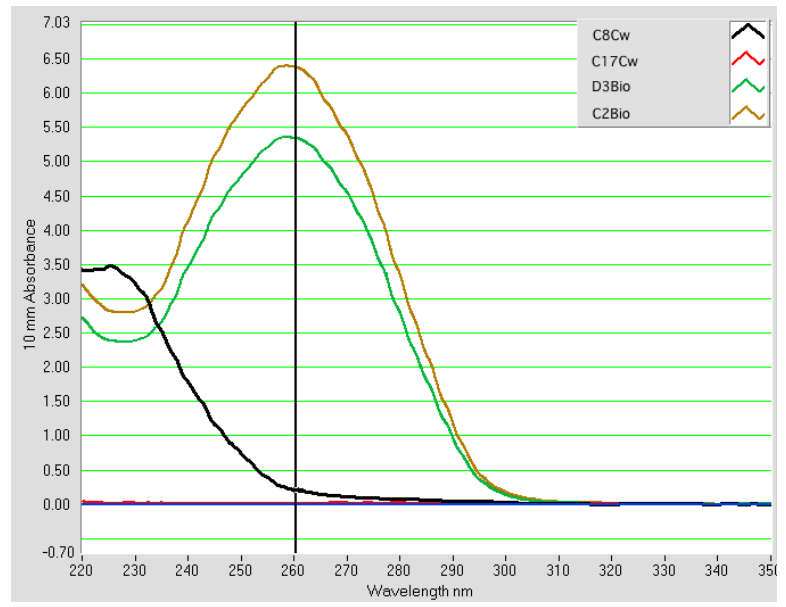

Figure 4.4.: NanoDrop Data Viewer- absorbance spectrum of Cryptosporidium DNA from cattle and biofilm samples. Absorbance at $260 \mathrm{~nm}$ is significantly lower for Cryptosporidiumpositive cattle sample ( $\mathrm{C} 8 \mathrm{Cw})$ compared to biofilm samples (D3Bio and $\mathrm{C} 2 \mathrm{Bio})$.

Cryptosporidium-negative sample $(\mathrm{C} 17 \mathrm{Cw})$ shows no absorbance.

\subsubsection{Temperature Gradients and Electrophoresis}

Temperature gradients utilizing DNA from cattle sample $\mathrm{C} 8 \mathrm{CW}$ were performed with the AccuStart $^{\mathrm{TM}}$ II PCR ToughMix and four different primer sets. Electrophoresis images are displayed in Figure 4.5. 
It was determined that the optimal annealing temperature for CPB-DIAG primers was $52.5^{\circ} \mathrm{C}$, which is slightly lower than previously described $\left(55^{\circ} \mathrm{C}\right)$ in Johnson et al. (1995). At $52.5^{\circ} \mathrm{C}$, CPB-DIAG primers showed a weak DNA band at the $\sim 435 \mathrm{bp}$ with non-specific binding present at $<100 \mathrm{bp}$ (Figure $4.5 \mathrm{a}$.). Similarly, the optimal annealing temperature for KLJ primers, was $52.5^{\circ} \mathrm{C}$, which is approximately the same as previously described $\left(53^{\circ} \mathrm{C}\right)$ in Jellison et al. (2002). At $52.5^{\circ} \mathrm{C}$, KLJ primers showed a very strong DNA band at the $\sim 1056 \mathrm{bp}$, with no non-specific binding at $<100 \mathrm{bp}$ (Figure $4.5 \mathrm{~b}$.). With regards to XIAO1 and XIAO2 primers, targeting the $1325 \mathrm{bp}$ and $835 \mathrm{bp}$, the optimal annealing temperature was $55^{\circ} \mathrm{C}$, as recommended by Xiao et al. (1999). At $55^{\circ} \mathrm{C}$, the nested primers showed very strong DNA bands with minimal to no nonspecific binding at $<100 \mathrm{bp}$ (Figure $4.5 \mathrm{c}$.).

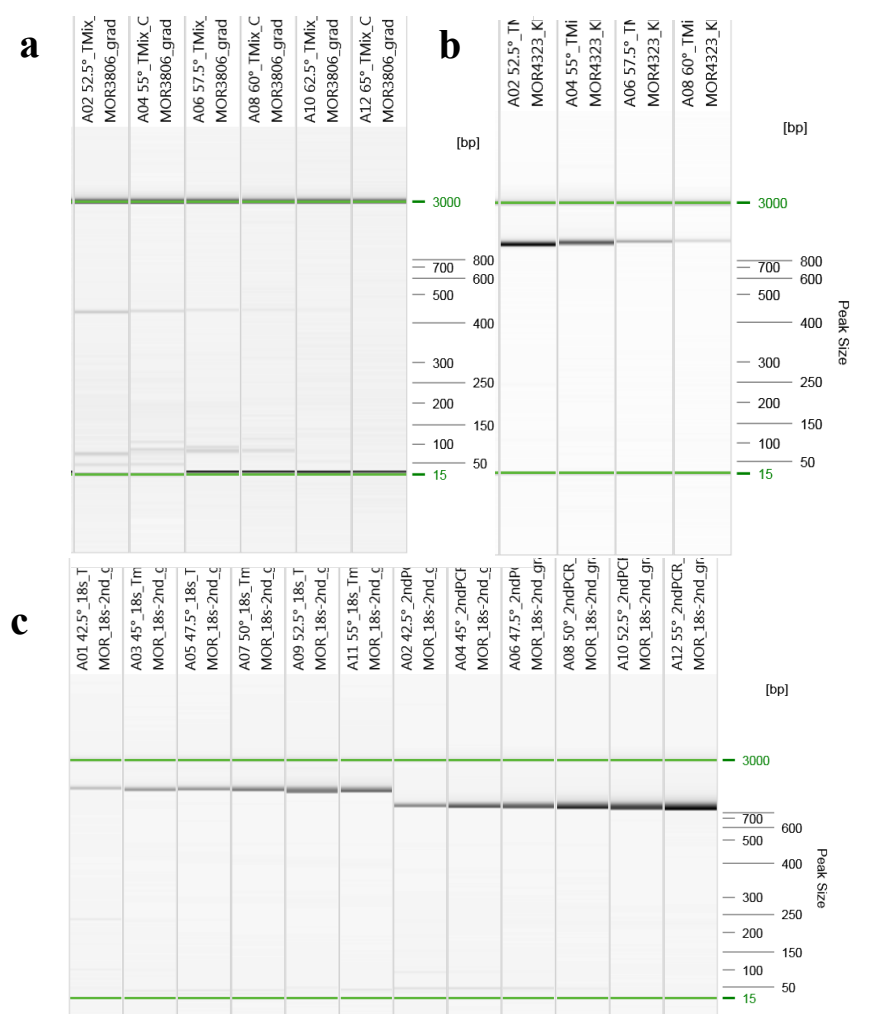

Figure 4.5.: Electrophoresis images from temperature gradients using selected CPB-DIAG (a), KLJ (b), and XIAO1 and XIAO2 (c) primers targeting the 18S rRNA. Temperature gradients were performed on extracted DNA from cattle sample C8Cw using AccuStart ${ }^{\mathrm{TM}}$ II PCR ToughMix. 


\subsection{Detection of Cryptosporidium from Biofilms}

\subsubsection{IFA Detection}

IFA microscopy was performed prior to PCR, to detect Cryptosporidium oocysts from biofilm samples. This is the first available research to investigate the occurrence of Cryptosporidium in surface water in Jamaica. Thus, it was important to perform initial screening to determine the pathogen's presence within the geographic location. A total of 24 biofilm-slides were collected from the river network in July 2016 and February 2017. Bright-field IFA microscopy with oil immersion and DAPI was used to analyze the biofilm suspensions for Cryptosporidium oocysts, according to EPA Method 1623. Seventy-eight replicated microscope slides were examined from Sites A, B, C, D, and E.

Morphological characteristics of oocysts including size, shape, outer-wall distinctiveness, and apple-green fluorescence was found to be consistent with those of Cryptosporidium spp. Oocysts size was predominantly 4-6 $\mu \mathrm{m}$ in diameter (Figure 4.6.), although some appeared as large as 7 $\mu \mathrm{m}$.

a

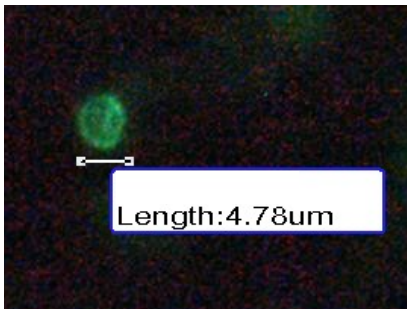

b

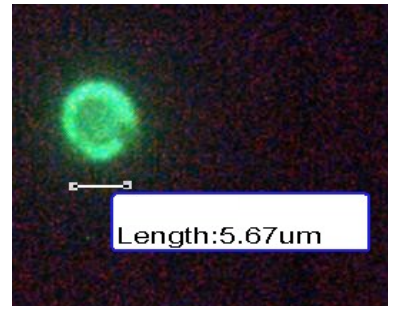

c

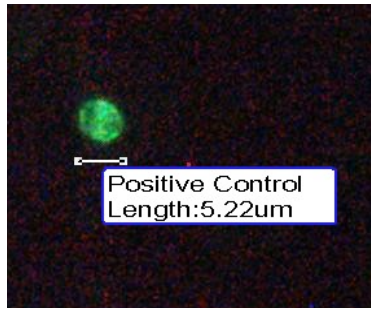

Figure 4.6.: Cryptosporidium oocysts detection from biofilms (a, b) and positive control (c) by IMS-IFA. The Crypt-a-Glo ${ }^{\text {TM }}$ Comprehensive Kit was used for immunofluorescence staining of oocysts, as recommended by EPA Method 1623. Magnification, 100X with immersion oil.

As shown in Table 4.5., every sampling site was positive for Cryptosporidium oocysts in at least one microscope-slide replicate. Nine $(11.5 \%)$ were positive by IFA with DAPI-visible nuclei, 
whereas $14(17.9 \%)$ were positive by IFA only. The frequency of oocysts detection was lowest at Site E, with 1 of $16(6 \%)$ samples positive for Cryptosporidium by IFA only. Site C had the highest frequency of detection, with 6 of 15 (40\%) samples positive for Cryptosporidium by IFA, of which 4 (27\%) were positive with DAPI-visible nuclei.

Table 4.5.: IFA Detection of Cryptosporidium Oocysts from Biofilms (Sites A-E)

\begin{tabular}{lcc}
\hline & $\begin{array}{c}\text { No. of IFA positive detections (No. of } \\
\text { slide replicates) }\end{array}$ \\
\cline { 2 - 3 } Site Ref. (Region) & Outer Shell & DAPI-Visible Nuclei \\
\hline Site A (Middle Quarters) & $5(16)$ & $3(16)$ \\
Site B (Cataboo) & $1(15)$ & $1(15)$ \\
Site C (Black River Capital) & $6(15)$ & $4(15)$ \\
Site D (Lacovia) & $1(16)$ & $1(16)$ \\
Site E (Bartons) & $1(16)$ & $0(16)$ \\
Total & $\mathbf{1 4}(\mathbf{7 8 )}$ & $\mathbf{9 ( 7 8 )}$ \\
\hline
\end{tabular}

\subsubsection{Nested-PCR Detection and Correspondence with IFA}

Following IFA, biofilms were analyzed for Cryptosporidium in replicates of 4 by nested-PCR. Initial samples collected in July 2016 from Sites A and B were not processed due to miscommunication of long-term storage at UWI, in which the samples were discarded. Figure 4.7. shows the electrophoresis images of the primary and secondary PCR products from Sites C, $\mathrm{D}$ and $\mathrm{E}$. In the primary round, there were no positive detections, as revealed by the absence of amplification at the $1056 \mathrm{bp}$. When the primary PCR products were employed as templates in the secondary round, visible bands appeared at the $\sim 435 \mathrm{bp}$. Second-round amplification was also observed in the positive control, while no amplification was observed in the negative control. 
It was determined that 4 of 12 biofilm samples were positive for Cryptosporidium; one sample was from Site C, and 3 samples were from Site D. There were no positive detections from Site E, located in the upper reaches of the river. Positive detections were only derived from the intersecting region of the Upper and Lower Morass (Site C), as previously shown in Figure 3.3., as well as near to the river outlet (Site D).

The correspondence between Cryptosporidium detection by means of IFA and nested-PCR was evaluated. Findings are provided in Table 4.6. Positive PCR products were obtained from 2 biofilm samples (C2Bio and D4Bio) that were also confirmed positive by IFA. Likewise, PCR detection was obtained from 2 IFA-negative samples (D1Bio and D3Bio). Results show inconsistencies between some IFA and PCR detections. In total, 6 of 12 samples were confirmed positive by either method.

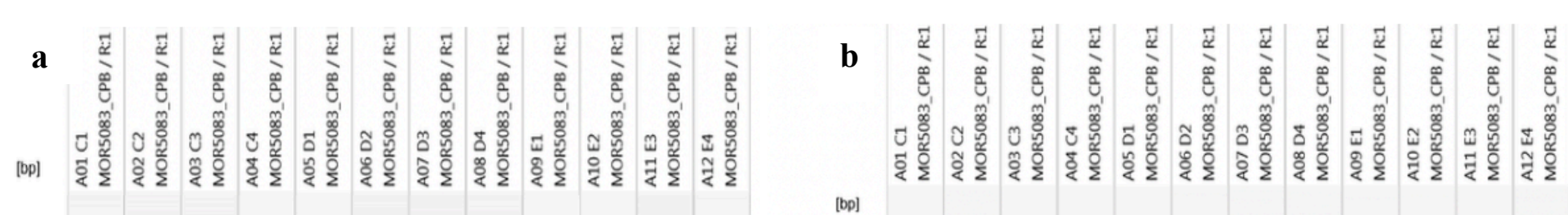

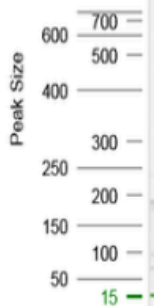

Figure 4.7. Primary (a) and secondary (b) 18S rRNA nested-PCR electrophoresis images of Cryptosporidium detection from biofilm samples, using KLJ (outer) and CPB-DIAG (inner) primers. Visible bands appear in the second round at the $\sim 435 \mathrm{bp}$. 
Table 4.6.: Comparison Between IFA and Nested-PCR Detection of Cryptosporidium from Biofilms

\begin{tabular}{|c|c|c|c|}
\hline Site Ref. (Region) & Sample ID & IFA & Nested-PCR \\
\hline \multirow{4}{*}{$\begin{array}{l}\text { Site C (Black } \\
\text { River Capital) }\end{array}$} & C1Bio & - & - \\
\hline & C2Bio & + & + \\
\hline & C3Bio & + & - \\
\hline & C4Bio & - & - \\
\hline \multirow{4}{*}{ Site D (Lacovia) } & D1Bio & - & + \\
\hline & D2Bio & - & - \\
\hline & D3Bio & - & + \\
\hline & D4Bio & + & + \\
\hline \multirow{4}{*}{ Site E (Bartons) } & E1Bio & - & - \\
\hline & E2Bio & - & - \\
\hline & E3Bio & - & - \\
\hline & E4Bio & + & - \\
\hline Total & - & 4 & 4 \\
\hline
\end{tabular}

\subsection{Detection of Cryptosporidium from Cattle}

\subsubsection{Comparative evaluation of MAF, ELISA and PCR techniques}

One hundred and nineteen fecal specimens were collected from 59 beef cattle and 60 dairy cattle, from 10 farms in February, March and October 2017. Because multiple techniques were employed for Cryptosporidium detection, the data was interpreted both collectively (based all three methods) and separately (based on individual method). In addition, diagnostic agreement between MAF microscopy and ELISA antigen test was evaluated as per Byrt (1996)'s classification scheme, previously summarized in Table 3.1 .

Through MAF staining, oocysts appeared a deep pink colour, with a size ranging between 4-6 
$\mu \mathrm{m}$ in diameter (Figure 4.8). For the ELISA antigen test, positive wells, which previously contained incubated specimens appeared yellow with an absorbance reading $\geq 0.150$ at $450 \mathrm{~nm}$. Results are summarized in Table 4.7. according to cattle age group, production type, and diarrheic symptoms. Complete data is available in Table C.II., Appendix C.

a

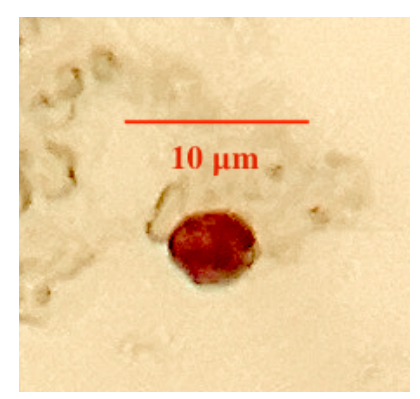

b

$10 \mu \mathrm{m}$

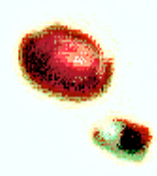

Figure 4.8.: Cryptosporidium oocysts detection from cattle sample 56DaCw (a) and positive control (b) by PBS-ether sedimentation and MAF staining. The Ward's ${ }^{\circledR}$ Chemistry AcidFast Stain Kit was used to stain oocysts, as per the CDC modified acid-fast staining procedure, 100X with immersion oil.

'Total' column displayed in Table 4.7. shows collective results, indicating that Cryptosporidium was detected in $39(33 \%)$ samples by any one or more technique. Meaning that, if a single method confirmed a positive detection, while the other methods were negative, the sample was deemed positive. When analyzing the data separately, based on individual method, MAF-staining microscopy shows highest positivity with 29 (24\%), followed by ELISA antigen test with 24 (20\%), and conventional PCR with 10 (12\%). Diagnostic agreement between MAF microscopy and ELISA antigen test, showed a slight $(k=0.39)$ agreement beyond chance. 
Table 4.7.: Cryptosporidium-positive Detections by MAF, ELISA and PCR in Relation to Age, Production Type, and Diarrheic Symptom

\begin{tabular}{|c|c|c|c|c|c|c|c|c|c|}
\hline \multirow{2}{*}{$\begin{array}{l}\text { Age/Production } \\
\text { Type/Diarrheic } \\
\text { Symptoms }\end{array}$} & \multirow{2}{*}{$\begin{array}{c}\text { No. of } \\
\text { Samples } \\
\text { Analyzed }\end{array}$} & \multicolumn{8}{|c|}{ No. of Cryptosporidium-positive Detections } \\
\hline & & MAF & $\begin{array}{c}\text { Freq. } \\
(\%)\end{array}$ & ELISA & $\begin{array}{c}\text { Freq. } \\
(\%)\end{array}$ & PCR & $\begin{array}{c}\text { Freq. } \\
(\%)\end{array}$ & Total $^{b}$ & $\begin{array}{c}\text { Freq. } \\
(\%)\end{array}$ \\
\hline 0 to $0.5 \mathrm{yr}$ & 23 & 5 & 22 & 5 & 22 & 2 & 9 & 6 & 26 \\
\hline 0.6 to 1 yr & 26 & 6 & 23 & 6 & 23 & 3 & 12 & 7 & 27 \\
\hline 2 to 3 yrs & 37 & 9 & 24 & 6 & 16 & 3 & 8 & 12 & 32 \\
\hline 4 to 5 yrs & 17 & 5 & 29 & 5 & 29 & 2 & 12 & 9 & 53 \\
\hline$\geq 6$ yrs & 16 & 4 & 25 & 2 & 13 & 0 & 0 & 5 & 31 \\
\hline Dairy & 60 & 18 & 30 & 17 & 28 & 7 & 12 & 25 & 42 \\
\hline Beef & 59 & 11 & 19 & 7 & 12 & 3 & 5 & 14 & 24 \\
\hline $\begin{array}{l}\text { Diarrheic (moderate } \\
\text { or severe) }\end{array}$ & 39 & 11 & 28 & 10 & 26 & 7 & 18 & 13 & 33 \\
\hline Non-diarrheic & 80 & 18 & 23 & 14 & 18 & 3 & 4 & 26 & 33 \\
\hline Total $^{\mathbf{a}}$ & 119 & 29 & 24 & 24 & 20 & 10 & 12 & 39 & 33 \\
\hline
\end{tabular}

${ }^{\text {a }}$ sum of all cattle specimens analyzed

${ }^{\mathrm{b}}$ sum of Cryptosporidium-positive detection by any one or more method

\subsubsection{Age-related, Production Type, and Diarrheic Symptom}

The prevalence of Cryptosporidium based on cattle age, demonstrates highest prevalence at age 4 to 5 years. Despite this observation, the differences in detection by individual method were not statistically significant $(p>0.05)$. Therefore, there were no age-related correlation between positive detections. Notably, there were no PCR detections from cattle $>6$ years of age.

The prevalence of Cryptosporidium detected in beef cattle compared to dairy cattle is shown in 
Figure 4.9. Cryptosporidium was confirmed in 14 (24\%) beef, compared to $25(42 \%)$ dairy by any one or more method. Collectively, positive detections were significantly higher in dairy cattle $(\mathrm{p}=0.04)$ compared to beef. When analyzing the data separately, 9,12 , and $5 \%$ of beef cattle, compared to 30,28 , and $12 \%$ of dairy cattle were positive by MAF, ELISA, and PCR, respectively. Statistical analysis determined that ELISA positives in dairy cattle were more prevalent than in beef cattle with significant correlation ( $\mathrm{p}=0.02$ ); conversely, MAF positives in dairy cattle showed no correlation $(\mathrm{p}=0.14)$.

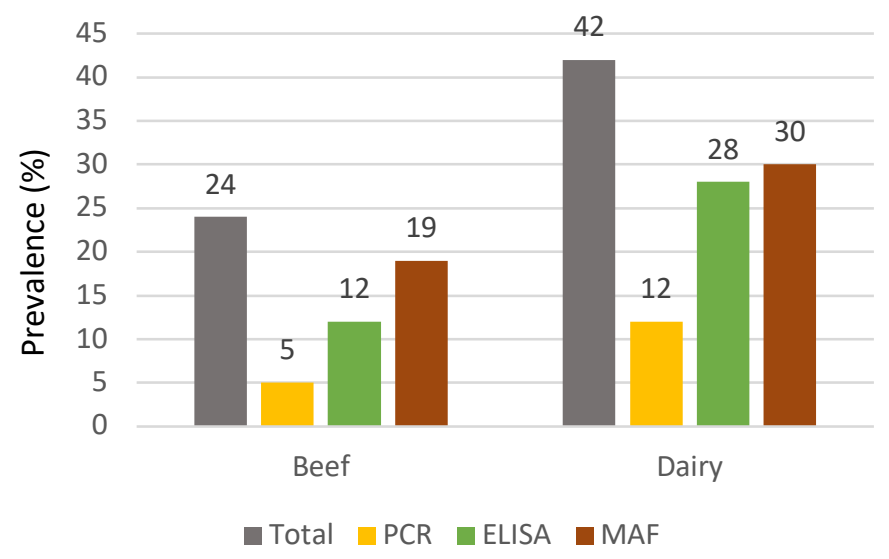

Figure 4.9.: Cryptosporidium detection by three different techniques in dairy and beef cattle. Total column refers to the number of positive detections by any one or more technique.

With regards to diarrheal symptoms, Cryptosporidium was detected by any one or more method from 13 of 39 (33\%) diarrheic cattle (experiencing moderate or severe symptoms) and 26 of 80 (33\%) non-diarrheic cattle. Therefore, both symptomatic and asymptomatic animals were carriers of the infection, showing no significant correlation with positive detections $(p=0.71)$. Notably, 7 of 10 (70\%) PCR-positive detections were from cattle that had moderate or serve diarrhea at the time of sample collection. Therefore, most molecular detection of Cryptosporidium was from cattle experiencing moderate-to-severe diarrhea. 


\subsubsection{Farm and Management}

Cryptosporidium detection per farm is presented in Figure 4.10. All farms except for F2 were positive for Cryptosporidium by any one or more method, with prevalence ranging from 10-56\%. It is important to note that only 5 fecal specimens were collected from F2. Farms F4 and F7 show highest positivity, with $10(50 \%)$ and $10(56 \%)$ cows infected by any one or more method, respectively.

In terms of PCR detections, it was determined that Cryptosporidium was present on 8 of 10 farms. Positive detection by PCR was not obtained from any of the 5 or 7 cattle that were housed on F2 and F5, respectively. Electrophoresis images of PCR-positive detections are provided in

Figure D.I., Appendix D.

$$
25
$$

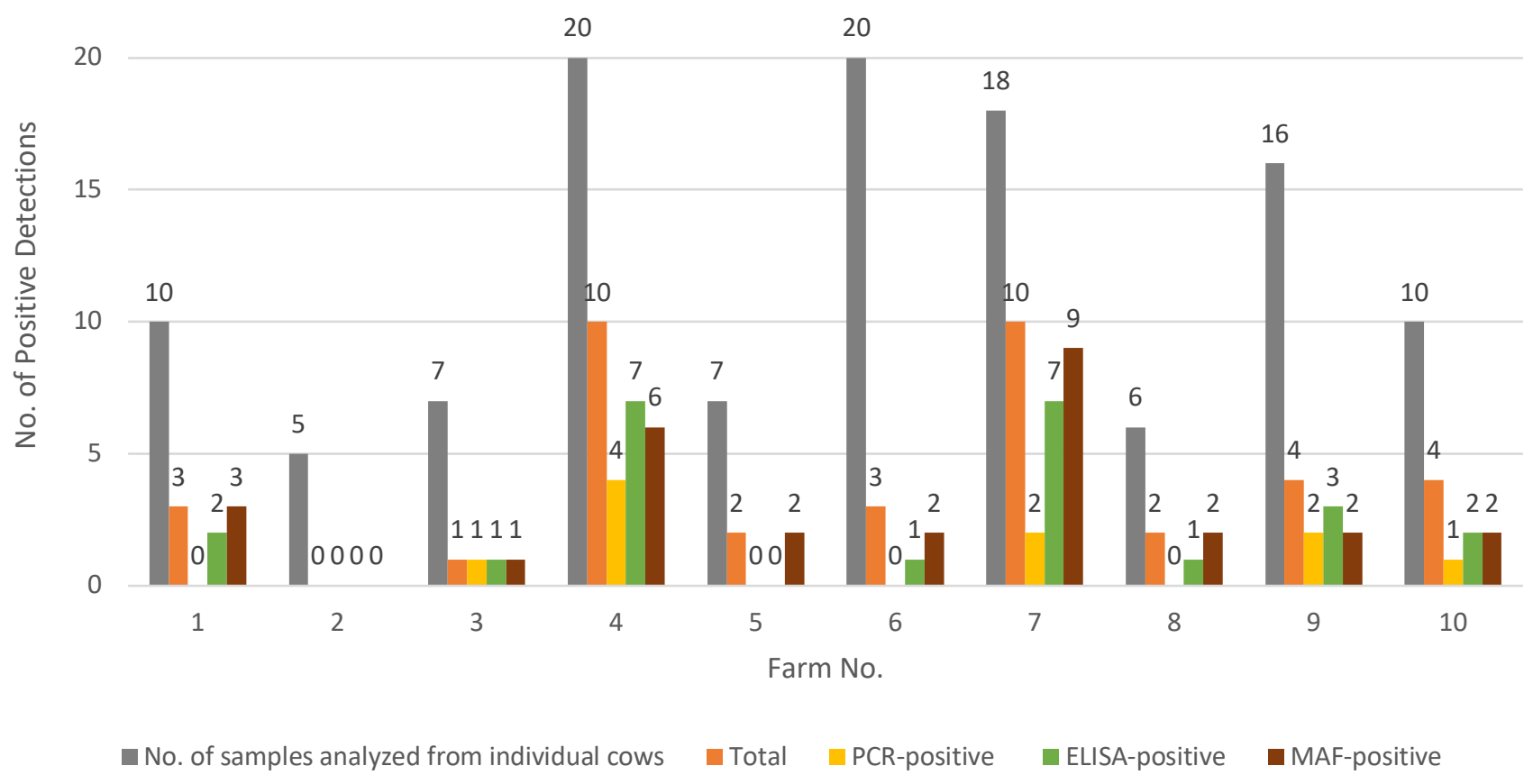

Figure 4.10.: Cryptosporidium detection by three different techniques in relation to farm. Total column refers to the number of positive detections by any one or more technique. 
With regards to farm management, an informal assessment was conveyed. Cattle on all ten farms were raised outdoors; either in a restricted space or freely roaming on grassland with large fenced enclosures. All cattle were potentially exposed to dogs, rodents and other wildlife animals. Farms consisting of beef cattle roamed the pasture freely or were spaced out. Dairy cattle were confined to small spaces to facilitate grain feeding and milking; thus, were exposed to each other's feces. Typically, pre-weaned calves on dairy farms were raised on concrete that was sheltered by zinc roofing. Unlike beef farms, it was common practice for dairy adult cattle to be separated from calves.

\subsection{Molecular Characterization and Phylogenetic Analysis of Cryptosporidium spp. and Subtypes}

\subsubsection{S rRNA Sequence Analysis and Identified Species/Genotypes}

Fourteen samples derived from biofilm $(n=4)$ and cattle $(n=10)$ sources were determined positive for Cryptosporidium by either conventional or nested-PCR. Positive detections were subjected to genotyping through sequence analysis of the $18 \mathrm{~S}$ rRNA gene. Clustal X alignment and NCBI BLAST confirmed 8 nucleotide sequences, which matched pre-existing genotypes in the GenBank database. The remaining 6 sequences had no match, presumably due to low quality of DNA and/or very low DNA targets present in the samples.

Genotyping of Biofilm Samples. Four biofilm samples, C2Bio, D1Bio, D3Bio, and D4Bio were confirmed positive by nested-PCR (Table 4.8.). Sequence analysis was successful and showed that all 4 positive detections had $\geq 98 \%$ homology with $C$. parvum (accession number: MF074701.1). This accession number matched a C. parvum genotype, which was previously identified in pre-weaned dairy calves in Shanghai China (Cai et al., 2017). 
Table 4.8.: Identified Cryptosporidium sp. from Biofilm Samples by Partial Sequencing of the 18S rRNA Gene

\begin{tabular}{lcccc}
\hline Site (Region) & Sample I.D. & Species & Identity \% & $\begin{array}{c}\text { GenBank } \\
\text { Accession No. }\end{array}$ \\
\hline $\begin{array}{l}\text { Site C (Black } \\
\text { River Capital) }\end{array}$ & C2Bio & C. parvum & 98 & MF074701.1 \\
Site D (Lacovia) & D1Bio & C. parvum & 98 & MF074701.1 \\
& D3Bio & C. parvum & 99 & MF074701.1 \\
& D4Bio & C. parvum & 98 & MF074701.1 \\
\hline
\end{tabular}

Genotyping of Cattle Samples. Ten cattle specimens were determined positive by conventional PCR, as displayed in the electrophoresis images of Figure D.I., Appendix D. DNA of these samples were also subjected to nested-PCR; however, only 4 were confirmed positive. DNA from samples — within \pm 50 bp of the 435 bp target—were also subjected to nested-PCR; however, no positives were observed. Electrophoresis imaging of the primary and secondary PCR products are provided in Figure D.II., Appendix D. In the primary round, samples $\mathrm{C} 34 \mathrm{Cw}$, $\mathrm{C} 53 \mathrm{Cw}, 105 \mathrm{Cw}$ were found positive as revealed by amplification at the $1325 \mathrm{bp}$. Primary PCR products were employed as templates in the secondary round, and visible bands appeared at the $\sim 835$ bp for $\mathrm{C} 13 \mathrm{Cw}, \mathrm{C} 34 \mathrm{Cw}, \mathrm{C} 53 \mathrm{Cw}, 105 \mathrm{Cw}$. Second-round amplification was also observed in the positive control, while no amplification was observed in the negative control.

Additionally, the concentration of DNA in each sample was evaluated using the intensity ranking scheme developed by Adamska et al. (2012). The intensity rankings obtained by conventional and nested-PCR are shown in Table 4.9. The intensity of DNA bands obtained with nested-PCR was either very strong (++++) or completely absent (-). Whereas, the intensity rankings obtained with conventional PCR varied: 3 were very strong, 1 was strong $(+++), 3$ were moderate $(++)$, 
and 3 were weak. Interestingly, nested-PCR using Xiao primers was more sensitive for higher concentration of DNA. However, for low DNA present in the cattle samples $(\sim 10 \mathrm{ng} / \mu \mathrm{l})$, conventional PCR using CPB-DIAG primers was more sensitive. Given these results, only conventional PCR products were sequenced. Table 4.9.: Intensity Rankings of Electrophoresis Bands, Comparing Conventional and
Nested PCR-positive Detections from Cattle

\begin{tabular}{lcc}
\hline Sample I.D. & $\begin{array}{c}\text { Results of } \\
\text { Conventional PCR }\end{array}$ & $\begin{array}{c}\text { Results of Secondary } \\
\text { Nested-PCR }\end{array}$ \\
\hline $\mathrm{C} 8 \mathrm{Cw}$ & +++ & - \\
$\mathrm{C} 13 \mathrm{Cw}$ & ++ & ++++ \\
$34 \mathrm{DaCw}$ & ++++ & ++++ \\
$40 \mathrm{DaCw}$ & + & ++++ \\
$46 \mathrm{BeCw}$ & ++ & - \\
$53 \mathrm{DaCw}$ & ++++ & ++++ \\
$56 \mathrm{DaCw}$ & + & - \\
$64 \mathrm{BeCw}$ & ++ & - \\
$78 \mathrm{Cw}$ & + & - \\
$105 \mathrm{Cw}$ & ++++ & - \\
\hline
\end{tabular}

$++++=$ very strong intensity of the DNA band

$+++=$ strong intensity of the DNA band

$++=$ moderate intensity of the DNA band

$+=$ weak intensity of the DNA band

$-=$ no visualization of the DNA band

As shown in Table 4.10., 18S rRNA sequencing identified C. parvum and C. hominis species from 4 of 10 positive cattle samples. The number of species and corresponding GenBank accession numbers are as follows: $C$. parvum was identified in 3 samples: 34DaCw (accession number: AB513881.1), 53DaCw (accession number: MF074701.1), and 105Cw (accession number: MF074701.1); C. hominis was identified in 1 sample: $\mathrm{C} 8 \mathrm{Cw}$ (accession number: 
KX342865.1).

C. parvum genotype detected in dairy cow $34 \mathrm{DaCw}$, matched with $99 \%$ homology to a sequence identified from dairy calves (AB513881.1) in Egypt (Amer et al., 2010). Similarly, C. parvum genotype detected in dairy cattle $105 \mathrm{Cw}$ and $53 \mathrm{DaCw}$, matched with $99 \%$ homology to the same isolate identified in all biofilm samples, which was identical to that of dairy calves from Shanghai, China (Cai et al., 2017). C. hominis genotype (KX342865.1) detected in beef cow $\mathrm{C} 8 \mathrm{Cw}$ was a direct submission to the GenBank database. The isolate matched with $78 \%$ homology to a genotype identified in human patients from New Delhi, India. Notably, all Cryptosporidium spp. were identified from cows that were $\leq 2$ years of age, including two preweaned calves; $\mathrm{C} 8 \mathrm{Cw}$ and $53 \mathrm{DaCw}$.

Table 4.10.: Identified Cryptosporidium spp. and Genotypes from Cattle by Partial Sequencing of the $18 \mathrm{~S}$ rRNA Gene

\begin{tabular}{lcccc}
\hline Farm I.D. & Sample I.D. & Species & Identity \% & $\begin{array}{c}\text { GenBank } \\
\text { Accession No. }\end{array}$ \\
\hline F3 & $105 \mathrm{Cw}$ & C. parvum & $88^{\mathrm{a}}$ & MF074701.1 \\
F4 & $\mathrm{C} 8 \mathrm{Cw}$ & C. hominis & $78^{\mathrm{a}}$ & KX342865.1 \\
& $\mathrm{C} 13 \mathrm{Cw}$ & - & - & - \\
& $40 \mathrm{DaCw}$ & - & - & - \\
& $46 \mathrm{BeCw}$ & - & - & - \\
F7 & $53 \mathrm{DaCw}$ & C. parvum & 99 & MF074701.1 \\
& $56 \mathrm{DaCw}$ & - & - & - \\
F9 & $34 \mathrm{DaCw}$ & C. parvum & 99 & AB513881.1 \\
& $78 \mathrm{Cw}$ & - & - & - \\
F10 & $64 \mathrm{BeCw}$ & - & - & - \\
& & & &
\end{tabular}

${ }^{a}$ unreliable identified Cryptosporidium spp. due to low \% identity 
Sequence analysis of the $18 \mathrm{~S}$ rRNA was also performed on PCR products that showed nonspecific bands. As previously mentioned, non-specific binding is a limitation of PCR assays, regarding Cryptosporidium detection from environmental samples (section 1.1.6.). This makes it difficult to assert representation of false-negatives. For example, of the 12 biofilm and 119 cattle samples, 8 non-specific bands were observed at the $\sim 485 \mathrm{bp}$, which is slightly higher than the targeted 435 bp. These samples include: E2Bio, C9Cw, C12Cw, C18Cw, C20Cw, C22Cw, $\mathrm{C} 24 \mathrm{Cw}$ and 22BeCw (refer to Figure D.I., Appendix D). Evidently, sequence analysis did not identify any Cryptosporidium spp. from these samples; although, Herbinix and Chania multitudinisentens bacteria were identified. Herbinix $s p$. are cellulose degrading bacteria (Koeck et al., 2015), while Chania multitudinisentens are bacteria found in soil landfill sites (Ee et al., 2016), which explains why they were consumed by cattle.

\subsubsection{Phylogenetic Analysis of $18 S$ rRNA Sequences}

Phylogenetic analysis confirming Cryptosporidium spp. and genotypes is provided in Figure 4.11. Multiple sequence alignment was performed on C. parvum (MF074701.1) and C. hominis (KX342865.1) genotypes, which were used for constructing the phylogenetic tree. The Neighbour-Joining method grouped all C. parvum isolates from this research with the designated reference species in 1 cluster ( $97 \%$ bootstrap support). Similarly, the $C$. hominis isolate was grouped with the reference species in 1 cluster (64\% bootstrap support). Multiple aligned sequences are provided in Figure 4.12., highlighting the variable region, which distinguishes $C$.

parvum and $C$. hominis. Chromatogram readings of the aligned sequences, showing good quality are provided in Figure E.I., Appendix E. 


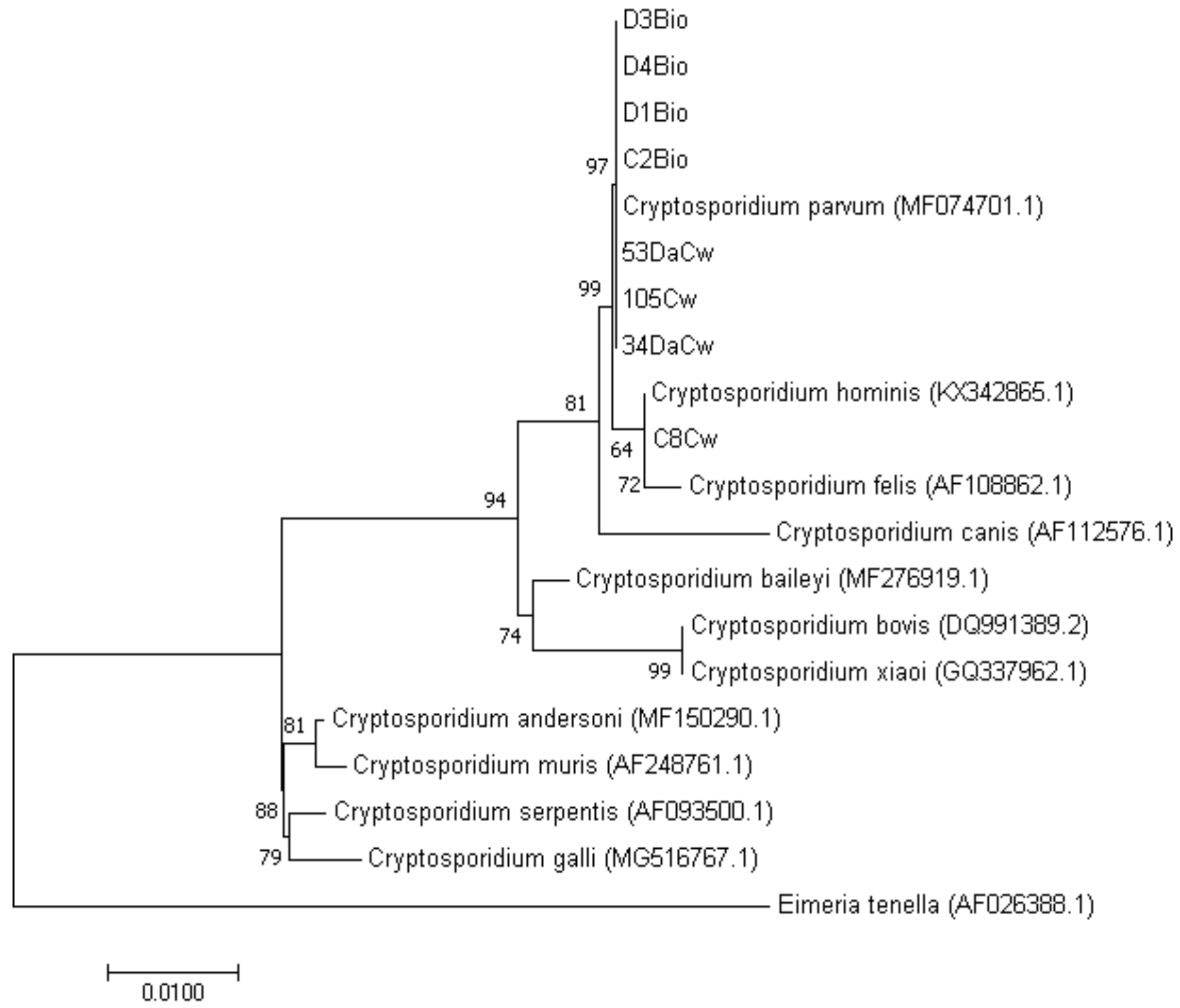

Figure 4.11. Phylogenetic relationships of Cryptosporidium isolates from cattle inferred by Neighbor-Joining analysis of 18S rDNA sequences in MEGA7. Reference species: C. parvum (MF074701.1) and C. hominis (KX342865.1). Outgroup species: Eimeria tenella (AF026388). 


C8Cw
105Cw
D3Bio
34DaCw
53DaCw
D4Bio
D1Bio
C2Bio
C.parvum
C.hominis

C8Cw
105Cw
D3Bio
34DaCw
53DaCw
D4Bio
D1Bio
C2Bio
C.parvum
C.hominis

$\mathrm{C} 8 \mathrm{Cw}$

$105 \mathrm{CW}$

D3Bio

$34 \mathrm{DaCw}$

$53 \mathrm{DaCW}$

D4Bio

D1Bio

C2Bio

C. parvum

C.hominis

$\mathrm{C} 8 \mathrm{CW}$

$105 \mathrm{CW}$

D3Bio

$34 \mathrm{DaCw}$

53DaCw

D4Bio

D1Bio

C2Bio

C.parvum

C.hominis

$\mathrm{C} 8 \mathrm{CW}$

$105 \mathrm{CW}$

D3Bio

$34 \mathrm{DaCw}$

53DaCw

D4Bio

D1Bio

C2Bio

C. parvum

C.hominis
TTTTAGWATATGAAATTTTACTTTGARAAAATTARAGKGCTTAARGCAGGCWTWTGCCTT TTTTAGWAWATRAAATTTTACTTTRARAAAATTARAGKGCTTAAAGCAGGCATATGCCTT TTTTAGTATATGAAATTTTACTTTGAGAAAATTAGAGTGCTTAAAGCAGGCATATGCCTT TTTTAGTATATGAAATTTTACTTTGAGAAAATTAGAGTGCTTAAAGCAGGCATATGCCTT TTTTAGTATATGAAATTTTACTTTGAGAAAATTAGAGTGCTTAAAGCAGGCATATGCCTT TTTTAGTATATGAAATTTTACTTTGAGAAAATTAGAGTGCTTAAAGCAGGCATATGCCTT TTTTAGTATATGAAATTTTACTTTGAGAAAATTAGAGTGCTTAAAGCAGGCATATGCCTT TTTTAGTATATGAAATTTTACTTTGAGAAAATTAGAGTGCTTAAAGCAGGCATATGCCTT TTTTAGTATATGAAATTTTACTTTGAGAAAATTAGAGTGCTTAAAGCAGGCATATGCCTT TTTTAGTATATGAAATTTTACTTTGAGAAAATTAGAGTGCTTAAAGCAGGCATATGCCTT

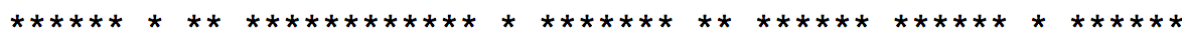
GAAMMCYCCAGCAKGRAAWAAWATWAAARATTTTTWTYTTTTITWTKGGTTCTAARAWAA GAAWACYCCASCAKGRAAWAAWATWAAARATTTTTATCTTTCIWATKGGTTCTAARATAA GAATACTCCAGCATGGAATAATATTAAAGATTTTTATCTTTCITATTGGTTCTAAGATAA GAATACTCCAGCATGGAATAATATTAAAGATTTTTATCTTTCITATTGGTTCTAAGATAA GAATACTCCAGCATGGAATAATATTAAAGATTTTTATCTTTCITATTGGTTCTAAGATAA GAATACTCCAGCATGGAATAATATTAAAGATTTTTATCTTTCITATTGGTTCTAAGATAA GAATACTCCAGCATGGAATAATATTAAAGATTTTTATCTTTCITATTGGTTCTAAGATAA GAATACTCCAGCATGGAATAATATTAAAGATTTTTATCTTTCTTATTGGTTCTAAGATAA GAATACTCCAGCATGGAATAATATTAAAGATTTTTATCTTTCTTATTGGTTCTAAGATAA GAATACTCCAGCATGGAATAATATTAAAGATTTTTATCTTT TITATTGGTTCTAAGATAA

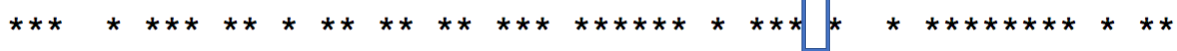

RAAWAAKGWTTAAWAGGGACRGTKGGGGGCWTTTKTWTTTAACAGTCRRAGGKGAAWTTY RAATAAKGATWAAWAGGGACAGTKGGGGGCWTTTGTATTTAACAGTCARAGGTGAAWTTC RAATAATGATTAATAGGGACAGTTGGGGGCATTTGTATTTAACAGTCAGAGGTGAAATTC RAATAATGATTAATAGGGACAGTTGGGGGCWTTTGTATTTAACAGTCAGAGGTGAAATTC RAATAATGATTAATAGGGACAGTTGGGGGCATTTGTATTTAACAGTCAGAGGTGAAATTC RAATAATGATTAATAGGGACAGTTGGGGGCATTTGTATTTAACAGTCAGAGGTGAAATTC RAATAATGATTAATAGGGACAGTTGGGGGCATTTGTATTTAACAGTCAGAGGTGAAATTC GAATAATGATTAATAGGGACAGTTGGGGGCATTTGTATTTAACAGTCAGAGGTGAAATTC GAATAATGATTAATAGGGACAGTTGGGGGCATTTGTATTTAACAGTCAGAGGTGAAATTC GAATAATGATTAATAGGGACAGTTGGGGGCATTTGTATTTAACAGTCAGAGGTGAAATTC $* * * * * * * * * * * * * * * * * * * * * * * * * * * * * * * * * * * * * * * * * * * *$

TTARWTTTGTTAAARACAAAYTAWKGCRAAAGCWTTTGCCARGWWTKTTTTCWTTAWTCA TWARATTTGTTAAARACAAACTAAKGCRAAAGCWTTTGCCAAGGATGTTTTCWTTAATCA TTAGATTTGTTAAAGACAAACTAATGCGAAAGCATTTGCCAAGGATGTTTTCATTAATCA TTAGATTTGTTAAAGACAAACTAATGCGAAAGCATTTGCCAAGGATGTTTTCATTAATCA TTAKATTTGTTAAAGACAAACTAATGCGAAAGCWTTTGCCAAGGATGTTTTCATTAATCA TTAGATTTGTTAAAGACAAACTAATGCGAAAGCATTTGCCAAGGATGTTTTCATTAATCA TTAGATTTGTTAAAGACAAACTAATGCGAAAGCWTTTGCCAAGGATGTTTTCATTAATCA TTAGATTTGTTAAAGACAAACTAATGCGAAAGCWTTTGCCAAGGATGTTTTCATTAATCA TTAGATTTGTTAAAGACAAACTAATGCGAAAGCATTTGCCAAGGATGTTTTCATTAATCA TTAGATTTGTTAAAGACAAACTAATGCGAAAGCATTTGCCAAGGATGTTTTCATTAATCA $* * * * * * * * * * * * * * * * * * \quad * * * * * * * * * * * * * * * * * * * * * * * * * * * *$

ARAACRAARGTRRGGGGAMCRAARACRAMCARATMCCGYCGWAKYCTTAACCAWAAACTA ARAACRAAAGTTAGGGGATCGAARACRATCAGATACCGTCGTAKTCTTAACCATAAACTA AGAACGAAAGTTAGGGGATCGAAGACGATCAGATACCGTCGTAKTCTTAACCATAAACTA AGAACGAAAGTTAGGGGATCGAAGACGATCAGATACCGTCGTAKTCTTAACCATAAACTA AGAACGAAAGTTAGGGGATCGAAGACGATCAGATACCGTCGTAKTCTTAACCATAAACTA AGAACGAARGTTAGGGGATCGAARACGATCAGATACCGTCGTAKTCTTAACCATAAACTA AGAACGAARGTTAGGGGATCGAAGACGATCAGATACCGTCGTAKTCTTAACCATAAACTA AGAACGAAAGTTAGGGGATCGAAGACGATCAGATACCGTCGTAKTCTTAACCATAAACTA AGAACGAAAGTTAGGGGATCGAAGACGATCAGATACCGTCGTAGTCTTAACCATAAACTA AGAACGAAAGTTAGGGGATCGAAGACGATCAGATACCGTCGTAGTCTTAACCATAAACTA

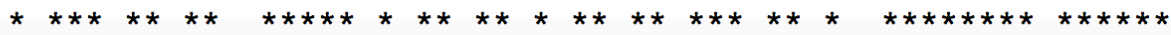

60

60

60

60

60

60

60

60

60

60

120

120

120

120

120

120

120

120

120

120

180

180

180

180

180

180

180

180

180

180

240

240

240

240

240

240

240

240

240

240

300

300

300

300

300

300

300

300

300

300 


\begin{tabular}{|c|c|c|}
\hline $\mathrm{C} 8 \mathrm{Cw}$ & TGCCAACTARARATKGG & 317 \\
\hline $105 \mathrm{CW}$ & TGCCAACTAGAGATTGG & 317 \\
\hline D3Bio & TGCCAACTAGAGATTGG & 317 \\
\hline $34 \mathrm{DaCw}$ & TGCCAACTAGAGATTGG & 317 \\
\hline $53 \mathrm{DaCw}$ & TGCCAACTAGAGATTGG & 317 \\
\hline D4Bio & TGCCAACTAGAGATTGG & 317 \\
\hline D1Bio & TGCCAACTAGAGATTGG & 317 \\
\hline C2Bio & TGCCAACTAGAGATTGG & 317 \\
\hline C.parvum & TGCCAACTAGAGATTGG & 317 \\
\hline C.hominis & TGCCAACTAGAGATTGG & 317 \\
\hline
\end{tabular}

Figure 4.12.: Clustal $X$ aligned nucleotide sequences obtained from PCR-positive biofilm and cattle samples, reference species: $C$. parvum (MF074701.1) and C. hominis

KX342865.1). Residues conserved in all species are shown (asterisks) and variable region highlighted (blue box).

\subsubsection{Gp60 Sequence Analysis and Identified Subtype}

Gp60 analysis to identify Cryptosporidium subtypes was important because phylogenetic analysis of $C$. parvum and $C$. hominis at the $18 \mathrm{~S}$ rRNA (hypervariable region) has shown to generate a similarity of 97.7\% (Morgan, Monis, Fayer, Deplazes, \& Thompson, 1999). Likewise, the percent identity of sequences from this study has shown a similarity of $99.68 \%$ amoung $C$. parvum and C. hominis detections (Figure 4.13.). Therefore, the two species are nearly identical at the $18 \mathrm{~S}$ rRNA locus, which could lead to misinterpretation.

\begin{tabular}{|c|c|c|c|c|c|c|c|c|c|c|}
\hline 1: $\mathrm{C} 8 \mathrm{Cw}$ & 100.00 & 84.86 & 77.92 & 78.23 & 78.23 & 78.55 & 78.55 & 77.92 & 77.29 & 77.60 \\
\hline $2: 105 \mathrm{Cw}$ & 84.86 & 100.00 & 88.33 & 88.64 & 88.64 & 88.33 & 88.33 & 88.33 & 87.70 & 87.38 \\
\hline 3: D3Bio & 77.92 & 88.33 & 100.00 & 99.68 & 99.37 & 99.37 & 99.37 & 99.37 & 99.37 & 99.05 \\
\hline $4: \quad 34 \mathrm{DaCw}$ & 78.23 & 88.64 & 99.68 & 100.00 & 99.05 & 99.05 & 99.05 & 99.05 & 99.05 & 98.74 \\
\hline 5: $53 \mathrm{DaCw}$ & 78.23 & 88.64 & 99.37 & 99.05 & 100.00 & 98.74 & 99.37 & 99.37 & 98.74 & 98.42 \\
\hline 6: D4Bio & 78.55 & 88.33 & 99.37 & 99.05 & 98.74 & 100.00 & 99.37 & 98.74 & 98.74 & 98.42 \\
\hline 7: D1Bio & 78.55 & 88.33 & 99.37 & 99.05 & 99.37 & 99.37 & 100.00 & 99.37 & 98.74 & 98.42 \\
\hline 8: C2Bio & 77.92 & 88.33 & 99.37 & 99.05 & 99.37 & 98.74 & 99.37 & 100.00 & 99.37 & 99.05 \\
\hline 9: C.parvum & 77.29 & 87.70 & 99.37 & 99.05 & 98.74 & 98.74 & 98.74 & 99.37 & 100.00 & 99.68 \\
\hline 10: C.hominis & 77.60 & 87.38 & 99.05 & 98.74 & 98.42 & 98.42 & 98.42 & 99.05 & 99.68 & 100.00 \\
\hline
\end{tabular}

Figure 4.13.: Percent Identity Matrix created by Clustal2.1, showing relationship between identified sequences with reference species. 


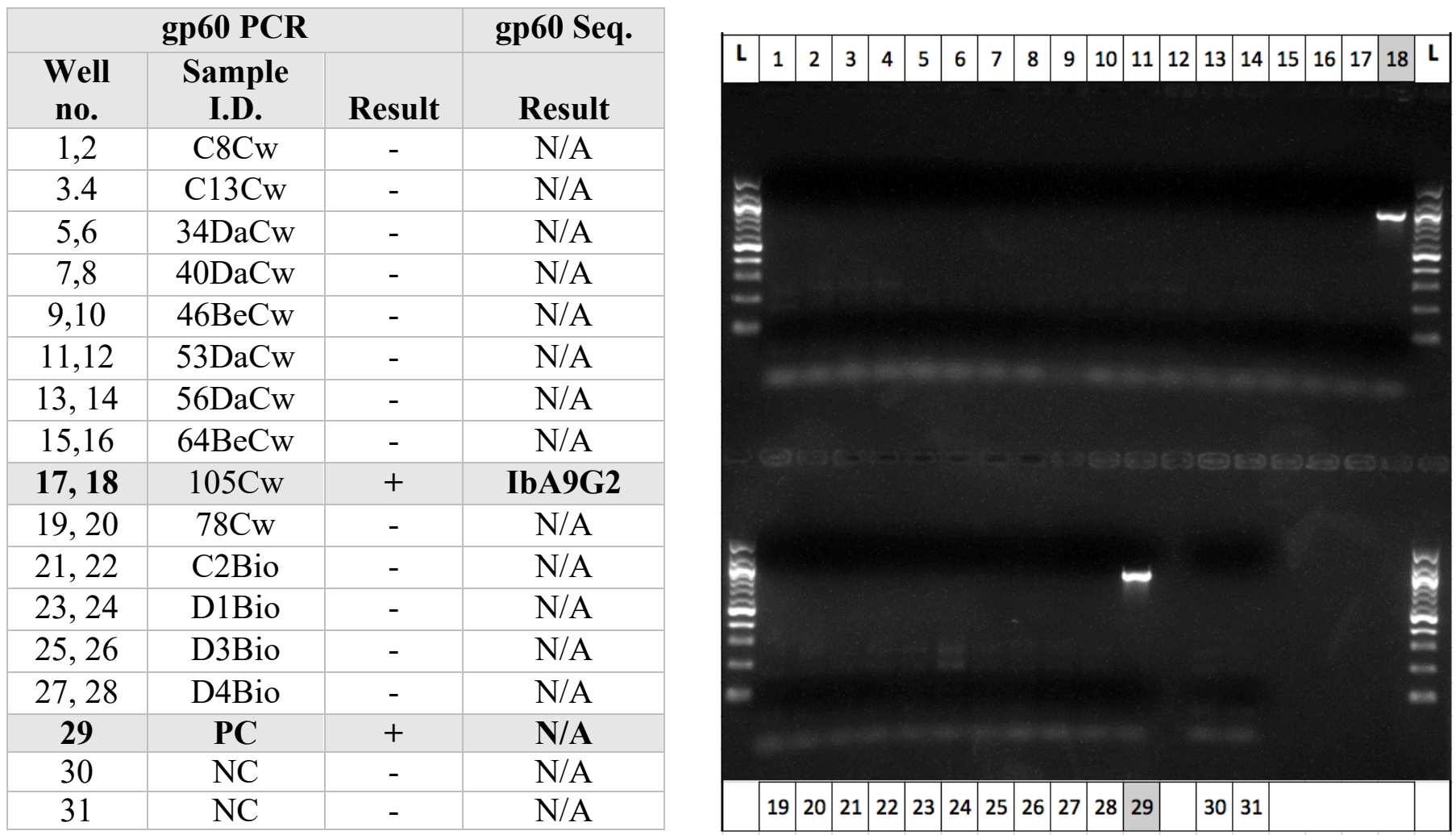

Figure 4.14.: Secondary Nested-PCR electrophoresis image of Cryptosporidium detection from cattle samples at the gp60 locus and corresponding subtype identification. $\mathrm{PC}$ is the positive control.

Here, gp60 analysis was performed by sequencing the highly polymorphic locus, to identify specific subtypes of Cryptosporidium-positive detections. In addition, gp60 analysis was performed to assess whether the subtypes are globally widespread, as well as known to cause zoonosis and/or severe symptoms in human populations. DNA from all 14 PCR-positive biofilm and cattle specimens were subjected to a nested-PCR that amplified a fragment of the gp60 gene. Figure 4.14. shows the electrophoresis imaging of the PCR products in the nested round. Visible bands only appear at a single sample, $105 \mathrm{Cw}$, as well as the positive control. Sequence analysis identified $C$. hominis subtype IbA9G2, corresponding with GenBank accession number AY166807 with 99\% homology. Previous studies have identified this particular subtype in humans (Pelayo et al., 2015), cattle (Razakandrainibe et al., 2018) and river water (Gertler et al., 
2015). It is important to note that the C. hominis subtype identified from sample $105 \mathrm{Cw}$ contradicts the previous identification of C. parvum at the $18 \mathrm{~S}$ rRNA locus. It is widely accepted that sequencing the gp60 gene, including the microsatellite region is a more specific target compared to the hypervariable 18s rRNA region. Thus, gp60 sequencing produces higher resolution data compared to $18 \mathrm{~S}$ rRNA, which often overlays different sequences. Moreover, $18 \mathrm{~S}$ rRNA sequencing of $105 \mathrm{Cw}$ corresponded to C. parvum with $88 \%$ homology; while gp60 sequencing of $105 \mathrm{Cw}$ corresponded to C. hominis with $99 \%$ homology. For this reason, $105 \mathrm{Cw}$ was believed to be C. hominis as determined through gp60 analysis. 


\section{Chapter 5: Discussion}

\subsection{Main Findings}

\subsubsection{Assessment of in situ biofilm roughness and thickness for Cryptosporidium detection}

In situ biofilm development was shown to be effective for Cryptosporidium detection in surface water. However, based on available literature, it is clear that the approach's success depends vastly on the ability for biofilms to obtain optimal structure that is favourable for oocysts capture and retention. Findings from the pilot study reveal that all biofilms reached a heightened stage of maturation suitable for oocysts entrapment, as determined by average thickness and maximum average thickness algorithms in COMSTAT2.

Here, biofilms attained an average thickness of 48.33 to $82.53 \mu \mathrm{m}$, with a maximum average thickness of 95.63 to $115.13 \mu \mathrm{m}$ following 3-weeks of incubation in creek water. These results are comparable to previous Cryptosporidium-biofilm investigations. Wolyniak et al. (2010) performed an in vitro study that portrayed a realistic depiction of oocysts entrapment in environmental biofilms collected from stream rocks. The biofilms were inoculated into a flowcell system, using C. parvum oocysts and filtered creek water as the flow-through medium. After eight days, the biofilms reached an average thickness between 25 and $42 \mu \mathrm{m}$, which showed to capture and sustain oocysts for at least 25 days. These average thickness measurements were slightly lower than determined in the present study; however, the biofilms were incubated for a shorter length of time.

Koh et al. (2013) also reported significant increases in oocysts recovery $(\mathrm{p}<0.001)$ as biofilm thickness increased from 21 to $105 \mu \mathrm{m}$. It is important to disclose that even immature biofilms as thin as $0.7 \mu \mathrm{m}$ (Koh et al., 2013) and $2.81 \mu \mathrm{m}$ (Searcy et al., 2006) have shown to capture 
oocysts within a flow-cell system. Despite these conflicting observations, optimization of oocysts entrapment seemly occurs as biofilms mature to a thickness of approximately $100 \mu \mathrm{m}$ (Koh et al., 2013), which was met by all biofilms from the pilot study.

Biofilms with $100 \mu \mathrm{m}$ thickness fulfil the penultimate stage of development, in which cell clusters are nonmotile and have reached a plateau (Davies et al., 1999; Sauer, Camper, Ehrlich, Costerton, \& Davies, 2002). At this stage, several layers of microorganisms have been added to the biofilms; thus, oocysts bury within denser regions, resulting in fewer detachments (Rogers \& Keevil, 1995; Warnecke, 2006). Similarly, a shielding-effect is produced on account of biofilm roughness. According to Sendamangalam (2012), biofilm roughness generates voids, creating quiescent zones (areas where fluid flow is absent) for pathogens to inhabit. In the pilot study, the mean roughness coefficient was $>1$. This is reflective of voids, also described as biofilm patchiness or heterogeneous formation; having minimal growth on some areas of the substrate (Merod et al., 2007; Murga, Stewart, \& Daly, 1994)

Overall, biofilm structure including thickness and surface roughness are critical factors to consider when monitoring Cryptosporidium spp. in environmental waters. Previous studies have shown that an increase in biofilm maturation correlates with oocysts embedment. However, it is important to note that excessive biofilm formation can result in sloughing; the dispersal of cell clusters from the interior portion of the biofilm. This process is completely randomized, often depending on the linear-flow velocity of water (Angles, Chandy, Cox, Fisher, \& Warnecke, 2007). Thus, it is unclear how widespread this phenomenon is amoung biofilms that have developed under different environmental conditions, let alone have diverse microbial composition. Nonetheless, studies have reported that sloughing occurs immediately after the 100 $\mu \mathrm{m}$ threshold (Sauer et al., 2002). In the present study, several biofilm segments reached a 
thickness $>115 \mu \mathrm{m}$ after 3-weeks (Table C.V, Appendix C); thus, it is presumed that sloughing may have occurred during the sample collection period. Based on these observations, a limiting factor of the current biofilm sampler prototype is that it may not be appropriate for predicting prevalence nor comparing point-source loading of Cryptosporidium oocysts in a given water source, as attempted here and in previous investigations (e.g., Barnes-Pohjonen, 2012; McLeod, 2011; PWD, 2014). Although, this may be applicable with greater understanding biofilm development in natural waters and oocysts recovery efficiency. Nonetheless, the technique remains beneficial for monitoring the absence or presence of Cryptosporidium, as well as determining its species and genotypes from environmental waters, which is discussed further in section 5.1.6.

\subsubsection{Biofilm sampling generates comparable oocysts recovery efficiencies to standardized filtration}

Biofilm sampling was shown to generate oocysts recovery rates that were comparable to Method 1623 , and in some cases, more efficient. It is important to acknowledge that the analysis of seeded oocysts from biofilms followed the same IMS-IFA procedure as Method 1623. The only difference was that REF coverslips were used to facilitate scanning through slides for oocysts quantification (section 3.1.5.).

Mean oocysts recovery from biofilms was $42.9 \% \pm 18.45$ and ranged between 23.9 and $68.7 \%$. These results are similar to Wolyniak et al. (2010), which determined oocysts recoveries from environmental biofilms between 24 and 65\%. Furthermore, a large-scale study recovered seeded oocysts from various water sources through filtration, using the same Dynabeads ${ }^{\mathrm{TM}}$ antiCryptosporidium kit. The study determined a mean oocysts recovery of $48.4 \% \pm 11.8$ from tap 
water; while from raw water, the recovery percentages fluctuated between 19.5 to $54.5 \%$ (McCuin and Clancy, 2003). Several other studies have confirmed fluctuating oocysts recoveries, between 1 and $61 \%$, from non-finished water sources including raw water, backwash and wastewater (Di Giovanni et al., 1999; Ferrari, Stoner \& Bergquist, 2006). Evidently, oocysts recovery by means of biofilm sampling is similar to that of environmental and wastewater filtration methods because they produce inconsistent results.

Inconsistent recovery rates from environmental sources are caused by several factors. Low efficiencies are reportedly due to significant losses of oocysts during the filtration step (Feng et al., 2003; Francy et al., 2004; Hu et al., 2004). In the present study, the biofilm sampling process eliminates the filtration step entirely; thus, the number of oocysts overlooked was potentially reduced. Low IMS recovery is also generated due to the presence of inhibitors. The presence of divalent cations and suspended solids in environmental and wastewaters influence the binding capacity of the antibody-coated IMS beads to oocysts (WHO, 2009). Ageing of oocysts also strips the epitopes from the outer shell of oocysts, further preventing antibody attachment. In such cases, IMS recovery from biofilms may have been disadvantageous because extensive attachment of natural organic matter (NOM) to oocysts has shown to result in steric repulsion (Dai \& Hozalski, 2002). Perhaps this occurred at Site E, wherein extensive NOM accumulation could be observed at the location during the sampling period (Figure G.I, Appendix G). In effort to mitigate this repulsive effect, the $\mathrm{pH}$ of all biofilm suspensions was adjusted to a neutralized charge, which has previously shown to optimize bead-binding capacity in water containing inhibitors (Dai \& Hozalski, 2002; Kuhn, Rock, \& Oshima, 2002).

With regards to suspended solids, turbidity is also an important factor to consider when performing IMS recovery of oocysts from biofilms. In deionized water, oocyst recovery is 
typically at an optimum range (between $76 \%$ to $83 \%$ ). However, at very high turbidity levels (e.g., $5000 \mathrm{NTU}$ ), the recovery of oocysts from water is usually $<35 \%$, depending on the kit used (Bukhari et al, 1998). In the present study, mean turbidity measured form the biofilm suspensions was $548.1 \pm 4.73 \%$ NTU. Presumably, this level did not affect IMS because 500 NTU is said to yield optimal results (Feng et al, 2003). Notably, several studies have also suggested that turbidity has minimal effect on oocyst recovery via IMS (Rochelle et al, 1999). Overall, divalent cations and suspended solids including NOM, potentially affected the IMS step of Method 1623 causing variable recovery rates that were unique to each biofilm culture. Whether these results were due to $\mathrm{pH}$, surface charge interactions, physical NOM barriers, or a combination of these factors needs to be investigated further.

\subsubsection{Concentrated and pure DNA extracted from oocysts derived from biofilm samples}

The isolation and enrichment of Cryptosporidium DNA, free of significant contaminates, is imperative for successful genotyping. According to Genomics Core Facility (n.d.), DNA concentration of purified PCR products should be at least $20 \mathrm{ng} / \mu \mathrm{l}$, to produce quality sequences, especially when using an automated sequencer, as in the present study. Through spectrophotometric analysis, a DNA concentration of $319.11 \mathrm{ng} / \mu \mathrm{l}$ and $267.13 \mathrm{ng} / \mu \mathrm{l}$ was determined from the purified PCR products of biofilms C2Bio and D3Bio, respectively, indicating that Cryptosporidium genomic DNA was present in the biofilms at very high concentrations. With regards to purity, the $\mathrm{A}_{260} / \mathrm{A}_{280}$ and $\mathrm{A}_{260} / \mathrm{A}_{230}$ ratios of sample $\mathrm{C} 2 \mathrm{Bio}$ and D2Bio were 2.27 and 2.24, respectively. As previously mentioned, the recommended "pure" range is typically between 1.8 and 2.2 (Thermo Scientific, 2009); thus, DNA extracted from these biofilm sources were very pure. 
A possible explanation for such abundant and pure DNA is that the outer shell of several oocysts remained intact while embedded within biofilms. This potentially aided in the maintenance of DNA integrity. It is believed that because biofilms have polysaccharide extracellular matrices in which oocysts are embedded, they are also capable of providing temporary relief against adverse environmental conditions, as well as mitigating factors such as aging, which cause the cells to crack open resulting in the disturbance of internal DNA (Anguish \& Ghiorse, 1997). Overall, these finding support the notion that biofilms serve as an active membrane continuously providing attachment and protection for pathogenic cells, including Cryptosporidium. Although, further research involving a larger sample size and oocysts viability is necessary to support this presumption.

\subsubsection{Biofilm sampling as an alternative to conventional filtration}

In the event of large outbreaks, analysis of multiple samples is necessary to provide spatial and

temporal assessment within a given region. Arguably, the high cost associated with filtration and IMS-microscopy-based detection, impedes on effective monitoring. Typically, identification of Cryptosporidium spp. from environmental waters involves collecting single grab-samples through filtration, in accordance with EPA Method 1623, then analyzing the samples through some form of genotyping assay. While informative, the current approach is often compromised due to poor recoveries, and limited snapshot data at the time of sample collection. Additionally,

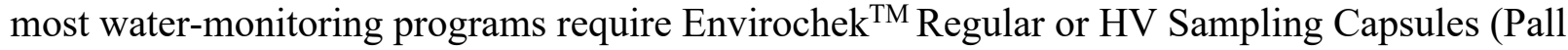
Corporation, Ann Arbor, MI), which are membrane filters that replace traditional string wound filters; thus, increase the capture and recovery efficiency of Cryptosporidium oocysts $>70 \%$ (Pall Corporation, 2017). These capsules however, currently cost \$149.98 USD each (VWR, 2017), and since multiple samples are required to obtain a comprehensive representation of 
Cryptosporidium spp. in natural waters (Hsu et al., 2002), the overall expenditure of the technique can be costly, especially for long-term investigations. To overcome these limitations, biofilm sampling has shown to yield a gradual representation of Cryptosporidium spp. passing through water columns over a given period of time, opposed to instantaneous grab-sampling. In addition, the technique has shown to be cost-effective compared to standardized filtration, as the apparatus itself is re-usable, and the only disposable expense is the polymeric material used to create the slides $(<\$ 1$ per slide) (refer to Figure F.I. in Appendix F).

In addition to cost, biofilm sampling generates comparable recovery efficiencies to standardized filtration because it concentrates oocysts in a similar manner to macroinvertebrates and filterfeeding sentinels. For example, researchers are constantly investigating alternate sources opposed to water filtrate for monitoring Cryptosporidium spp. in aquatic environments. Reboredo-Fernández et al. (2014) homogenized benthic macroinvertebrates including nymphs, water bugs and larvae from 9 rivers in Galicia, Spain and found Cryptosporidium oocysts in $12.5 \%$ of the samples via IFA-microscopy. Likewise, shellfish have become widely accepted as bioindicators of fecal contaminants, including bacteria, viruses and parasites (Potasman, Paz, \& Odeh, 2002). Numerous studies have shown that shellfish including oysters, mussels, clams, and cockles, share the ability of bioaccumulating Cryptosporidium oocysts, while retaining them in their hemolymph, digestive glands, gastrointestinal tracts and on gills (Graczyk, Fayer, Lewis, Trout, \& Farley, 1999; Guiguet Leal et al; 2008; Miller et al., 2005; Staggs et al., 2015). In many cases, Cryptosporidium spp. have been detected from shellfish, even when they were undetected from surrounding waters through filtration (as cited in Miller et al., 2005). Moreover, various Cryptosporidium spp. have been identified from shellfish through PCR and gene sequencing applications, providing epidemiological context to point-source transmission, as well as genotypes that are endemic in human populations (Gomez-Bautista et al., 2000; Miller et al., 
2006; Staggs et al., 2015). Work presented in this dissertation therefore, demonstrates that the same research outcomes can be achieved through biofilm sampling. Even further applications may be achieved, given the widespread prevalence of biofilms in various aquatic environment; whereas shellfish are limited to ambient waters.

\subsubsection{High prevalence of Cryptosporidium infections in dairy cattle with potential association to rearing system}

Cryptosporidium has been found in many livestock animals worldwide; however, infections are reportedly endemic in cattle species (Santín \& Trout, 2008). In the present study, Cryptosporidium was detected in 14 (24\%) beef cattle compared to 25 (42\%) dairy cattle. These findings were based on the combined results of three different detection methods, which revealed significant prevalence in dairy cattle $(\mathrm{p}=0.04)$. This pattern was also observed separately by MAF, ELISA, and PCR methods, as 9, 12, and 5\% of beef cattle, compared to 30, 28, and $12 \%$ of dairy cattle were positive, respectively. However, it is important to note that based on statistical analysis, only ELISA positive detections in dairy cattle showed higher prevalence than in beef cattle with significance ( $\mathrm{p}=0.02$ ); conversely, MAF positives in dairy cattle showed an insignificant correlation $(\mathrm{p}=0.14)$. A possible explanation is the slight diagnostic agreement beyond chance between MAF microscopy and ELISA antigen test. This highlights the importance of using multiple methods for detecting Cryptosporidium from cattle feces, especially since oocysts concentrations can be low and inhibitors are often abundant (i.e., cellulose inhibitors due to grass consumption).

Results demonstrating a high prevalence of Cryptosporidium infections amoung dairy cattle is supported by several epidemiological studies. Gong et al. (2017) determined that the rate of 
infection in dairy cattle was more prevalent than in beef cattle (Gong et al., 2017). While another study based in the Canadian provinces of Alberta, Saskatchewan and British Columbia, found that mortality rates in beef calves increased by $30 \%$, usually when dairy calves were introduced to the beef herds during calving season (Olson, Ralston, O’Handley, Guselle, \& Appelbee, 2003). Moreover, a large-scale study, which identified C. parvum in 1253 calves from Czech Republic, determined that pre-weaned dairy calves had highest prevalence of infection up to $56.5 \%$ per farm; while only three cases of C. parvum oocysts shedding was found in pre-weaned calves from beef farms (Kváč, Kouba, \& Vítovec, 2006). Therefore, previous studies support the observation that dairy cattle have a higher prevalence of Cryptosporidium infection than beef cattle.

The disparities between Cryptosporidium infection in dairy versus beef cattle may be attributed to differences in rearing systems. In the present study, an informal assessment of farms revealed that beef cattle typically roamed the pasture more freely or were spaced out. Whereas, dairy cattle were confined to small spaces to facilitate grain feeding and milking; thus, were exposed to each other's feces. Garro, Morici, Utgés, Tomazic, and Schnittger (2016) investigated the occurrences of Cryptosporidium in dairy calves from Argentina and suggested that this phenomenon is in part due to conditions of artificial rearing systems. Because dairy cattle are commonly raised in confinement, they are predisposed to rapid infection and subsequent reinfection. On the other hand, beef cattle are commonly kept outside in open ranges, which spreads the infection relatively slower. These claims are further supported by McAllister, Olson, Fletch, Wetzstein, and Entz (2005), which investigated the prevalence of Cryptosporidium detection in 49 dairy and beef farms across Ontario and British Columbia, Canada. The study observed that dairy calves allowed outside confinement were reportedly less likely to be infected by Cryptosporidium compared to their counterparts kept in barns. The differences in the rearing 
and production systems accounted for the $63.3 \%$ infection rate in dairy cattle compared to the $18.4 \%$ in beef cattle. Therefore, preventative measures pertaining to housing are crucial for mitigating the spread of cryptosporidiosis within dairy herds.

Adequate housing of cattle involves several strategies. Cattle should be kept in hygienic and spacious environments to prevent the risk of spreading the disease. Animal feeds and water are prone to contamination by oocysts; therefore, regular cleaning of pen floors and troughs, while minimizing contact between animals may aid in reducing spread. It is also essential to separate the feed from defecation areas. In the present study, most dairy farms had regular cleaning routines; although it appeared challenging for farmers to keep the feed separated from feces given the overcrowded feed-lots. Thus, beside cleaning, farms should mitigate overcrowding, as it has shown to increase animal contact, while presenting the challenge of upkeeping sanitary measures (Ghazy, Abel-Shafy, \& Shaapan, 2016). Furthermore, sanitary or hygienic practices is most important for young dairy calves, which are more susceptible to the cryptosporidiosis compared to older animals. It is recommended to use concrete flooring for young calves because it makes cleaning easier, which was observed to be a common practice on most of dairy farms in the present study.

Overall, despite being discovered over 100 years ago, Cryptosporidium continue to be amoung the most difficult pathogens to control on cattle farms. As shown in the present study, Cryptosporidium was widespread on 9 of 10 farms, with highest prevalence in dairy cattle. Currently, there is no vaccine or drug available to combat bovine cryptosporidiosis, and our knowledge of host-pathogen interactions in cattle is very limited. Thus, future research should focus on understand prevalence and risk factors for oocysts shedding, to effectively monitor and implement control measures that can help mitigate the parasites' transmission within herds and 
to the surrounding environment.

\subsubsection{C. parvum and C. hominis isolates identified in biofilm and cattle samples}

In the remote geographical region of St. Elizabeth, Jamaica, a low species diversity consisting of C. hominis and $C$. parvum genotypes was detected in biofilm and cattle samples. Generally, $C$. hominis is host-specific because it transmits primarily within human populations. On the other hand, C. parvum is considered anthropozoonotic because it transmits from both humans and animals including ruminants, domestic pets, and wildlife.

Identification of Cryptosporidium sp. from biofilm samples. C. parvum was the only species identified from the biofilm samples through 18S rRNA sequencing. These results are similar to that recently reported in Haiti; wherein, C. parvum was the sole species identified from 15 of 16 water samples collected from natural waters. The researchers suggested that the samples contained either a single species or multiple species that were undetected (Damiani et al., 2013).

If a single species was present in the biofilm samples, then there is a possibility that clonality contributed to low species diversity. Clonality effects the diversity of Cryptosporidium spp. because it causes a dominant mutant to exist within a population. This is true for species developed in isolation, which potentially occurred in the remote region of St. Elizabeth, Jamaica. For example, species that develop in isolation may experience genetic pool monotony, leaving the fate of a single, beneficial mutation to exist within a population. Thus, the interspecies competition may have lead to the extinction of diverse mutants (Ndaoa et al., 2013). Isolation also means that hosts are not exposed to different incoming species. Thus, sexual reproduction becomes an exchange of genetic material that is the same amoung members of the same species. 
Previous studies have demonstrated that confinements in hosts have the same effects as confinement to geographic locations in relation to Cryptosporidium epidemiology (Wang et al., 2014).

Another explanation of low species diversity is that there were undetected species due to the use of basic molecular tools. In such cases, superimposition may have occurred during gene sequencing. For example, sequencing of the $18 \mathrm{~S}$ rRNA does not account for species that co-exist within a single sample. In many cases, the sequences were unreadable due to the overlaying of different species, which can be observed from the chromatogram readings shown in Figure E.I., Appendix E. Also, a combination of clonality and superimposition may have occurred, where $C$. parvum was so prevalent that it masked the DNA from any other Cryptosporidium spp. coexisting in the same sample. Moreover, a single gene target was assessed at the 18S rRNA in this research, which does not account for other genes that may have been more prevalent.

\section{Identification of Cryptosporidium spp. and subtype from cattle specimens. Four species of} Cryptosporidium are typically found in cattle: C. parvum, C. bovis, C. ryanae and C. andersoni (Fayer, Santin, \& Trout, 2008; Fayer, Santin, \& Xiao, 2005). In the present study, C. parvum was identified in 2 cattle ( $\geq 98 \%$ homology); while $C$. hominis was identified in 2 cattle. It is important to note that one of the $C$. hominis isolates matched subtype IbA9G2 with 99\% homology; while the other $C$. hominis isolate match with only $78 \%$ homology, indicating unreliable identification.

It was not surprising to have identified $C$. parvum from cattle because as previously mentioned, this species is most widespread amoung a diverse group of animals. According to Jamaica's Rural Agricultural Development Authority (RADA), St. Elizabeth is the leading livestockproducing parish in the country, having over 25,000 registered farmers (RADA, 2017). The 
livestock rearing systems includes: goats, sheep, hogs, cattle and horses—all of which are known carriers of C. parvum (Fayer \& Xiao, 2008). Thus, given the outdoor environment, in which cattle often cohabit with other livestock animals on the same agricultural land, the potential exposure of C. parvum was plausible. In contrast, it was surprising to have identified C. hominis from cattle because it is generally associated with human infections. Nonetheless, in low prevalence, earlier studies have identified C. hominis in cattle (through 18s rRNA sequencing) from Malawi (Banda, Nichols, Grimason, \& Smith, 2009), Korea (Park et al., 2006), Scotland (Smith et al., 2005) and India (Feng et al., 2007).

Furthermore, the specific $C$. hominis IbA9G2 subtype was recently reported in symptomatic and asymptomatic calves in France (Razakandrainibe et al., 2018). This marked the first reported bovine case of gp60 C. hominis connected to the Ib subtype family. Interestingly, Gatei et al. (2008) also identified a C. hominis subtype from the Ib family in 71\% HIV infected persons in Jamaica. The study confirmed that the subtype was geographically distinct to the Jamaican population, which potentially explains why this particular subtype family was found in cattle from the Black River watershed. Additionally, human cases of the IbA9G2 subtype was previously reported in humans from Australia (O’Brien, McInnes, \& Ryan, 2008; Waldron, Ferrari, \& Power, 2009), French Guiana (Mosnier et al., 2018), Europe (Gertler et al., 2015) and Cuba (Pelayo et al., 2008). Furthermore, Chalmers et al. (2008) conducted a case-control study and sequenced 115 isolates from the gp60 locus to further understand the geographical transmission of $C$. hominis. The study confirmed that subtype IbA9G2 was mainly linked to Europe because it was not connected to outside travel.

Finally, the IbA9G2 subtype was connected to a waterborne outbreak in Germany between May and June 2013, where an abnormal 24 cases of cryptosporidiosis occurred in the city of Halle. 
The cases were investigated further, and only $C$. hominis of IbA9G2 subtype was detected from the patients' stool. The outbreak was attributed to extensive river flooding, which damaged sewage systems, resulting in an overflow into drinking water sources (Gertler et al., 2015). This study provides insight, potentially explaining how a $C$. hominis subtype was ingested by cattle in the Black River watershed. Presently, there are no existing sewage treatment plants in the parish of St. Elizabeth, Jamaica. The primary method of sewage disposal is hand-dug soak-away pits (Miller, Waite, \& Harlan, 2001). Therefore, the absence of a centralized or formal sewage treatment system may have contributed to cattle exposure to human feces, particularly during times of heavy rain activity.

Overall, fecal contamination due to human effluent and livestock feces have led to several Cryptosporidium outbreaks in the past (Putignani \& Menichella, 2010). The transmission of oocysts from humans and terrestrial animals to surface waters pose significant health risks to those utilizing the ecosystem. Although groundwater is the predominant drinking water source in St. Elizabeth, the Black River watershed is also used regularly for crop irrigation, recreational swimming and tourism. Thus, the presence of C. parvum and C. hominis in surface water and cattle feces suggests that both agricultural and human wastewater are potential sources contributing to Cryptosporidium infections in St. Elizabeth. However, additional work investigating human cases of cryptosporidiosis in the region is needed to draw a direct correlation to environmental sources.

\subsection{Limitation and Future Work}

Limitations and future work of this dissertation were alluded to in section 5.1. This includes: 1) sample size, allocation and timing; 2) sophistication of molecular tools; 3) no recount of 
viability; and 4) limited information available in the region.

1. Sample size, allocation and timing is a limitation because it impeded on the ability to obtain vital information regarding Cryptosporidium population structure with geographical, temporal and statistical significance. The dataset as a whole consisted of biofilm samples collected from 5 sites, and 119 cattle feces collected from 10 farms; however, information for each sample location was restricted due to low occurrences of Cryptosporidium species and genotypes. Also, the samples were collected at different times, which contributed to insufficient epidemiological design. Although restrictions in funding made it unfeasible to increase sample size, it is also recognized that an alternative approach would have been to limit sample allocations to only a few water sources and farms within closer proximity. This would have potentially increased detection numbers within a smaller geographical location and timeframe, and thus help to draw stronger connections between Cryptosporidium transmission from cattle farms to nearby water sources. Future research should aim to examine these relationships at a more refine level, in order to gather geospatial information for source tracking Cryptosporidium spp. throughout the watershed.

2. Sophistication of molecular tools is a limitation because it restricted the ability to identify co-existing Cryptosporidium spp. within a single biofilm sample or co-infections within individual cattle specimens. It also restricted the sensitivity of molecular detection from environmental samples containing naturally low numbers of oocysts and inhibitors. As previously mentioned, superimposition was believed to have occurred during $18 \mathrm{~S}$ rRNA and gp60 sequencing, where dominant species, in this case $C$. parvum and $C$. hominis, potentially overlaid the less dominant species (section 5.1.6.). Identification of 
less dominant species requires sophisticated molecular tools such as multi-locus sequencing (the use of multiple gene targets) and PCR-RFLP to distinguish co-existing species or co-infections. In addition, advance molecular applications such as cloning or in vitro inoculation of positive samples into mice would have increased concentrations; thereby facilitate the identification of Cryptosporidium species and genotypes. In hindsight, improved identification of less dominant species using the same molecular tools employed in this dissertation, could have involved increasing the number of replicates per source. For example, successive sampling (e.g., sampling the same group of cattle multiple times over the duration of one month), would have accounted for intermittent infections.

3. No account of viability is a limitation because it did not provide information about whether the positive detections were viable; therefore, could cause infection. To a certain degree, knowledge of species and genotypes assemblages in surface water provides an understanding as to whether the population structure poses an immediate health risk. However, to be more precise, viability is an important factor to consider, especially when detecting Cryptosporidium from environmental sources because many oocysts could have been dead or damaged, thus do not pose significant threat to public health. Therefore, from a public health standpoint, viability is important to consider when making assessments about risk. Future research may investigate viability through various means including CC-PCR, bio-assays in mice, and immunofluorescence staining using vital dyes in conjunction with confocal microscopy to visualize occurrence of excystation.

4. Limited information available in the region presented major knowledge gaps regarding 
host range and transmission dynamics. With no existing data on whether certain water sources accounted for outbreaks in the past, as well as records of livestock and wildlife causing zoonosis, it becomes difficult to assert whether cattle contributed as a point source of Cryptosporidium spp. loading into the Black River network. Even though the same C. parvum isolate detected in biofilm was found in cattle with $>99 \%$ homology, other livestock animals within the watershed could have accounted for the transmission. Future work should therefore aim to establish databases of past anthropocentric occurrences of cryptosporidiosis, as well as species and genotypes detected from livestock and wildlife in Jamaica.

\subsection{Summary of Research Contributions and Outlook}

In situ biofilm sampling can produce biofilms at optimal thickness and roughness for oocysts entrapment in environmental waters. However, the randomized influence of sloughing and oocysts attachment to biofilms most likely was impacted by various factors including biofilm architecture, water chemistry and velocity, the diversity of organisms, and the presence of NOM. Thus, the biofilm sampling method warrants further investigation to obtain a better understanding of the complexity of biofilm development in natural waters and oocysts recovery efficiencies. This would make the technique more applicable for determining Cryptosporidium prevalence in a given region. Nonetheless, the technique was shown to provide informative data about the absence and presence of Cryptosporidium. Additionally, the technique provided a source of Cryptosporidium oocysts, which was effectively used for further molecular characterizations.

In terms of molecular analyses, initial research carried out in this dissertation used selected 
primers previously published, as a basis for optimizing Cryptosporidium detection from environmental sources. Through spectrophotometric analysis and DNA gradient tests, a systematic process, which enhanced the sensitivity and specificity of conventional and nestedPCR was initiated. The procedure developed in this dissertation has shown to be effective for isolating enriched Cryptosporidium DNA from biofilm samples, thus is valuable for advanced genotyping assays that require pure and concentrated DNA. Moreover, verification of DNA purity was confirmed through successful $18 \mathrm{~S}$ rRNA sequencing. Results have demonstrated that in conjunction with IMS, the genotyping assay generated the specificity and sensitivity needed for analyzing Cryptosporidium from biofilms. The use of this approach led to the $18 \mathrm{~S}$ rRNA sequencing of a single C. parvum isolate in 4 PCR-positive biofilm samples. However, the downfall was the techniques inability to differentiate diverse Cryptosporidium species and genotypes, especially with further gp60 analyses. Therefore, with further refinement, the technique can potentially lead to the wide use of comparative genomics in epidemiological investigations of Cryptosporidium contamination in various water sources.

With regards to Cryptosporidium detection and gene sequencing from cattle feces, C. hominis and C. parvum were identified, which marks the first reported cases of Cryptosporidium spp. found in cattle feces from Jamaica. The identified species are the two most commonly found in humans; thus, this research provides evidence that there is potential zoonotic transmission occurring in the region. In addition, most Cryptosporidium detections were from dairy animals, which further supports the notion that there is a high prevalence of infections in dairy cattle compared to beef cattle. In the present study, restricted space of dairy herds was believed to have accounted for the higher prevalence of infections. Although, there are many other plausible factors to consider, thus further epidemiological research is necessary to draw this correlation. 
Overall, our understanding of Cryptosporidium as an infectious protozoan parasite is influenced by previous researchers who have independently developed different methods for detecting and identifying Cryptosporidium species and genotypes, while drawing relationships between what was observed to 'real-life' occurrences. Although these novel methods have provided significant insight into Cryptosporidium biology and host-parasitic interactions, they have also led to a plethora of publications aiming to enhance the techniques, making it extremely difficult to establish a standardized method.

Many studies have stressed that standardization is imperative, to improve water-monitoring regimes from environmental sources (Cacci \& Chalmers, 2016; Karanis et al., 20006; Díaz-Lee et al., 2015). However, it seems that no matter what innovative technique is employed for Cryptosporidium purification and detection, available technologies will continue to yield, to some degree, a level of inefficiency due to the abundance of inhibitors and naturally low number of oocysts found in environmental samples. Thus, the broader scope of this research calls for a shift in conventional monitoring frameworks for Cryptosporidium and alike species from environmental waters. Research should stray away from looking to adopt a single, standardized method; to incorporate a more integrated approach involving identification from multiple sources using several techniques. As long as there's an understanding of the sensitivity and specificity of each method, this collective approach could potentially be more applicable for obtaining a compressive representation of Cryptosporidium species and genotypes that exist in aquatic ecosystems.

As previously mention, the purpose of developing the biofilm sampler technique was to offer an alternative method to work in conjunction with multiple techniques applied to a group of sources that are known reservoirs or vectors of Cryptosporidium spp. Whether this group contains 
biofilms, shellfishes, benthic macroinvertebrates, the idea is to improve recovery of oocysts from these natural sources because they are more reflective of the species and genotypes that persist at a sampling location compared to filtered grab-samples. Therefore, identification of

Cryptosporidium species and genotype from a well-planned set of environmental sources, may aid in watershed monitoring, and thus develop interventions going forward. 


\section{Appendices}

\section{Appendix A: Suppliers and Catalogue Numbers of Reagents and Kits}

Table A.I.: Reagents and Kits for IMS-IFA Staining

\begin{tabular}{ll}
\hline Reagents/Kits & Supplier (Cat. No.) \\
\hline Dynabeads $^{\mathrm{TM}}$ anti-Cryptosporidium Kit & Thermo Fisher Scientific, Waltham, MA (73011) \\
Crypt-a-Glo $^{\mathrm{TM}}$ G/C Direct Comprehensive Kit & Waterborne, Inc., New Orleans, LA (A400FLK) \\
Hydrochloric acid & Thermo Fisher Scientific, Waltham, MA (SA54-1) \\
Sodium hydroxide solution & Thermo Fisher Scientific, Waltham, MA (SS266-1) \\
Methanol & Anachemia Canada, Lachine, QC (ANAC56902-360) \\
Nuclease-free Water & New England Biolabs Ltd., Ipswich, MA (12931S) \\
\hline
\end{tabular}

Table A.II.: Reagents and Kit for PBS-Ether Sedimentation and MAF Staining

\begin{tabular}{ll}
\hline Reagents/Kit & Supplier (Cat. No.) \\
\hline Phosphate Buffer Saline (PBS) Tablets & VWR, Mississauga, ON (97062-730) \\
$\begin{array}{l}\text { VWR Life Science Water, Sterile, Purified, } \\
\text { Molecular Biology Grade }\end{array}$ & VWR, Mississauga, ON (L0201-1000) \\
Ethyl Ether & Thermo Fisher Scientific, Waltham, MA (E134-1) \\
Methanol & Anachemia Canada, Lachine, QC (ANAC56902-360) \\
Ward's® Chemistry Acid-Fast Stain Kit & VWR, Mississauga, ON (470038-940) \\
C. parvum slide & VWR, Mississauga, ON (470181-942) \\
C. parvum Non-viable Positive Control & Waterborne Inc., New Orleans, LA (PC101) \\
\hline
\end{tabular}

Table A.III.: Reagents for Biofilm Staining

\begin{tabular}{ll}
\hline Reagents & Supplier (Cat. No.) \\
\hline $\begin{array}{l}\text { SYTO } \\
\text { Stain } 9 \text { Green Fluorescent Nucleic Acid }\end{array}$ & Thermo Fisher Scientific, Waltham, MA (S34854) \\
Streptavidin Texas Red & \\
\hline
\end{tabular}


Table A.IV.: Reagents and Kit for ELISA

\begin{tabular}{ll}
\hline Kit & Supplier (Cat. No.) \\
\hline CRYPTOSPORDIUM TEST & TechLab, Blacksburg, VA (PT5014) \\
\hline
\end{tabular}

Table A.V.: Reagents and Kits for DNA Extraction

\begin{tabular}{ll}
\hline Reagents/Kit & Supplier (Cat. No.) \\
\hline Chelex ${ }^{\circledR} 100$ Resin & $\begin{array}{l}\text { Bio-Rad Laboratories, Inc., Hercules, Germany } \\
(1421253)\end{array}$ \\
QIAamp ${ }^{\circledR}$ Fast DNA Stool Mini Kit & Qiagen, Hilden, Germany (51604) \\
\hline
\end{tabular}

Table A.VI.: Reagents and Kits for Conventional/Nested-PCR and Electrophoresis

\begin{tabular}{ll}
\hline Reagents/Kits & Supplier (Cat. No.) \\
\hline Primers & ACGT Corporation, Toronto, ON \\
AccuStart & Quantabio, Beverly, MA (95142-800) \\
FastStart $^{\mathrm{TM}}$ Taq DNA Polymerase, dNTPack & Sigma-Aldrich, St. Louis, MO (4738357001) \\
GoTaq ${ }^{\circledR}$ DNA polymerase & Promega, Madison, WI (M3005) \\
DNA Gel Loading Dye (6X) & Thermo Fisher Scientific, Waltham, MA (R0611) \\
TAE Buffer & Thermo Fisher Scientific, Waltham, MA (15558042) \\
Ultra-Pure $^{\mathrm{TM}}$ agarose & Invitrogent $^{\mathrm{TM}}$, Carlsbad, CA (15510-027) \\
Ultra-Pure $^{\mathrm{TM}}$ Ethidium Bromide & Thermo Fisher Scientific, Waltham, MA (15585011) \\
QIAxcel DNA Fast Analysis Kit & Qiagen, Hilden, Germany (929008) \\
\hline
\end{tabular}

Table A.VII.: Reagents and Kit for PCR Purification

\begin{tabular}{ll}
\hline Reagents/Kit & Supplier (Cat. No.) \\
\hline QIAquick® PCR Purification Kit & Qiagen, Hilden, Germany (28104) \\
\hline
\end{tabular}


Table A.VIII.: Reagents and Kits for Gene Sequencing

\begin{tabular}{ll}
\hline Reagents/Kit & Supplier (Cat. No.) \\
\hline $\begin{array}{l}\text { BigDye }{ }^{\mathrm{TM}} \text { Terminator v3.1 Cycle } \\
\text { Sequencing Kit }\end{array}$ & Thermo Fisher Scientific, Waltham, MA (43374556) \\
\hline
\end{tabular}




\section{Appendix B: Maps of the Black River Watershed}

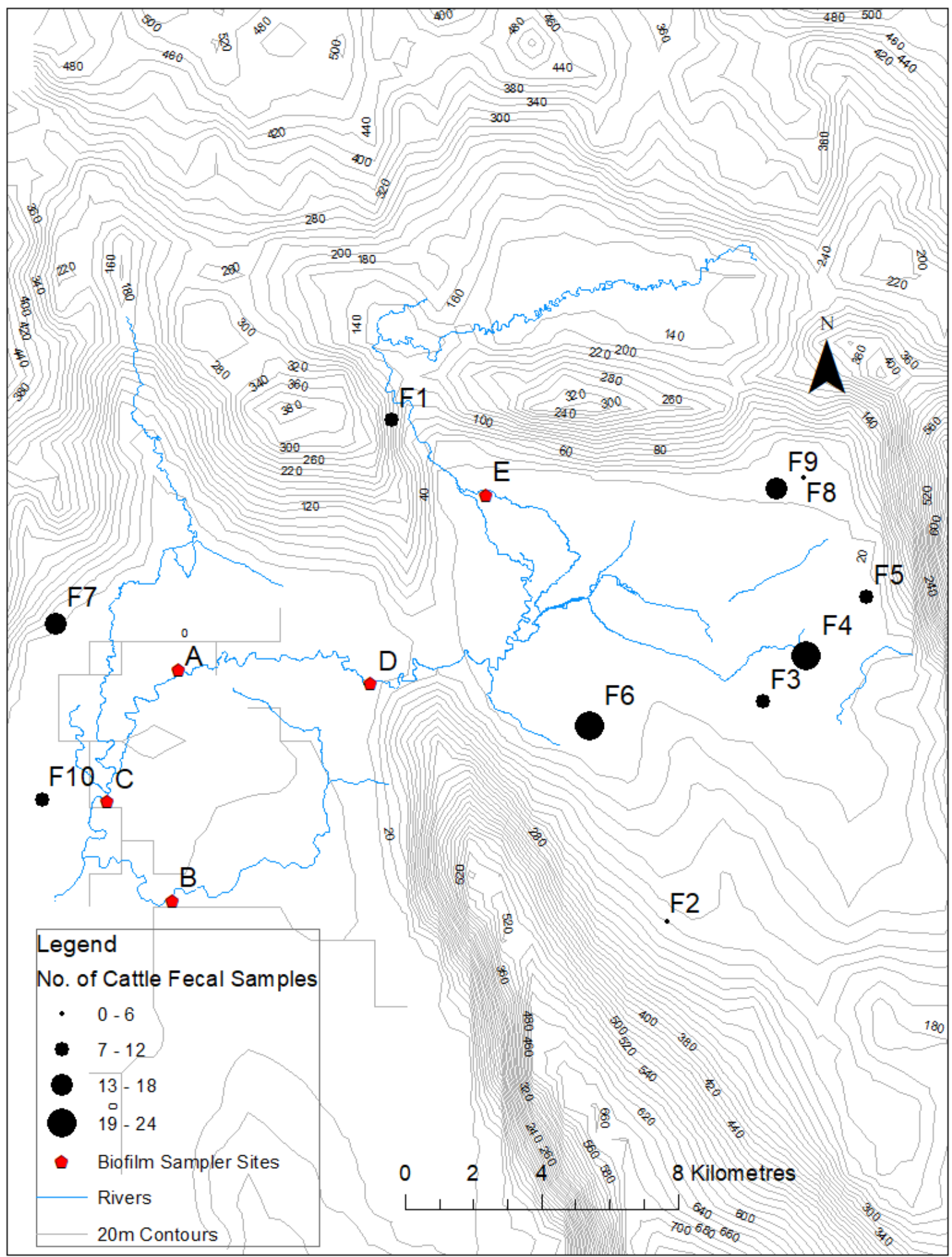

Figure B.I.: Topography map of the Black River watershed and sampling locations created in ARCMap10. 

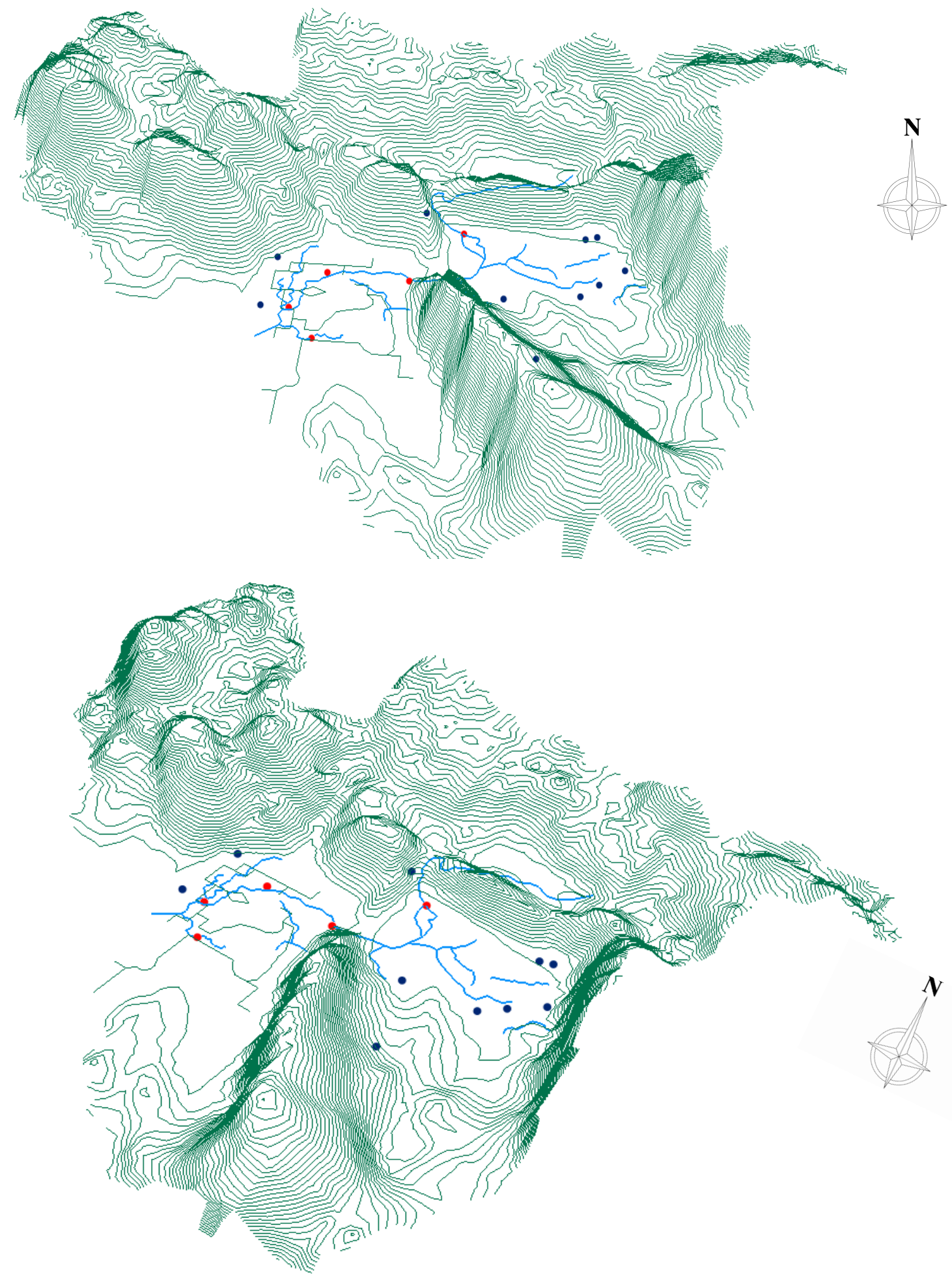

Figure B.II.: 3D Map of the Black River watershed from different point of views and sampling locations created in ARCScene10. Biofilm sampler sites (red) and cattle farms (blue). 


\section{Appendix C: Complete Data Sets}

Table C.I.: Qualitative Records of Cattle Sample Collection

\begin{tabular}{|c|c|c|c|c|c|c|}
\hline $\begin{array}{l}\text { Farm I.D. and } \\
\text { Coordinates } \\
\end{array}$ & Cow I.D. & \begin{tabular}{|l|}
$\begin{array}{l}\text { Collection } \\
\text { Date }\end{array}$ \\
\end{tabular} & \begin{tabular}{|l|} 
Approx. \\
Age (yrs) \\
\end{tabular} & Sex & Breed (Production Type) & \begin{tabular}{|l|} 
Diarrheal \\
Symptoms
\end{tabular} \\
\hline \multirow{10}{*}{$\begin{array}{l}\text { F1 (Lat: 18.14292; } \\
\text { Long: -77.7578) }\end{array}$} & $\mathrm{C} 1 \mathrm{Cw}$ & $02 / 28 / 17$ & 3 & $\mathrm{~F}$ & Indian (Beef) & Moderate \\
\hline & $\mathrm{C} 2 \mathrm{Cw}$ & $02 / 28 / 17$ & 4 & $\mathrm{~F}$ & Indian mixed with common (Beef) & None \\
\hline & $\mathrm{C} 3 \mathrm{CW}$ & $02 / 28 / 17$ & 2 & $\mathrm{~F}$ & Indian mixed with common (Beef) & None \\
\hline & $\mathrm{C} 4 \mathrm{Cw}$ & $02 / 28 / 17$ & $0.5^{\mathrm{a}}$ & M & Brahman mixed with common (Beef) & None \\
\hline & $\mathrm{C} 5 \mathrm{Cw}$ & $02 / 28 / 17$ & 1 & $\mathrm{~F}$ & Indian (Beef) & None \\
\hline & $\mathrm{C} 6 \mathrm{Cw}$ & $02 / 28 / 17$ & 2.5 & M & Brahman mixed with common (Beef) & Moderate \\
\hline & $77 \mathrm{Cw}$ & $10 / 26 / 17$ & $0.5^{\mathrm{a}}$ & $\mathrm{F}$ & Indian (Beef) & None \\
\hline & $91 \mathrm{CW}$ & $10 / 26 / 17$ & 3 & $\mathrm{M}$ & Brahman mixed with common (Beef) & Moderate \\
\hline & $94 \mathrm{CW}$ & $10 / 26 / 17$ & 1.5 & $\mathrm{~F}$ & Indian mixed with common (Beef) & None \\
\hline & $111 \mathrm{Cw}$ & $10 / 26 / 17$ & 2.5 & $\mathrm{~F}$ & Indian (Beef) & Moderate \\
\hline \multirow{5}{*}{$\begin{array}{l}\text { F2 (Lat: } \\
\text { 18.017333; Long: } \\
-77.6853 \text { ) }\end{array}$} & $\mathrm{C} 7 \mathrm{Cw}$ & $02 / 28 / 17$ & 1 & $\mathrm{M}$ & Red Poll mixed with common (Beef) & None \\
\hline & $107 \mathrm{Cw}$ & $10 / 26 / 17$ & 4 & $\mathrm{~F}$ & Indian (Beef) & None \\
\hline & $33 \mathrm{BeCw}$ & $03 / 13 / 17$ & $0.1^{\mathrm{a}}$ & M & Red Poll mixed with common (Beef) & None \\
\hline & $103 \mathrm{CW}$ & $10 / 26 / 17$ & 1 & $\mathrm{~F}$ & Brahman (Beef) & None \\
\hline & $112 \mathrm{Cw}$ & $10 / 26 / 17$ & $0.5^{\mathrm{a}}$ & $\mathrm{M}$ & Red Poll mixed with common (Beef) & None \\
\hline \multirow{7}{*}{$\begin{array}{l}\text { F3 (Lat: } \\
\text { 18.072382; Long: } \\
-77.659925)\end{array}$} & $\mathrm{C} 19 \mathrm{Cw}$ & $02 / 28 / 17$ & 4 & $\mathrm{~F}$ & Jamaica Hope (Dairy) & None \\
\hline & $\mathrm{C} 20 \mathrm{Cw}$ & $02 / 28 / 17$ & 6 & $\mathrm{~F}$ & Jamaica Hope (Dairy) & None \\
\hline & $100 \mathrm{CW}$ & $10 / 26 / 17$ & 8 & $\mathrm{~F}$ & Holstein (Dairy) & None \\
\hline & $102 \mathrm{Cw}$ & $10 / 26 / 17$ & 11 & $\mathrm{~F}$ & Holstein (Dairy) & Moderate \\
\hline & $105 \mathrm{Cw}$ & $10 / 26 / 17$ & 1 & $\mathrm{~F}$ & Holstein (Dairy) & Moderate \\
\hline & $106 \mathrm{Cw}$ & $10 / 26 / 17$ & 2 & M & Red Poll (Beef) & Moderate \\
\hline & $114 \mathrm{CW}$ & $10 / 26 / 17$ & 1 & $\mathrm{M}$ & Red Poll (Dairy) & Severe \\
\hline \multirow{12}{*}{$\begin{array}{l}\text { F4 (Lat: } \\
\text { 18.083909; Long: } \\
-77.648551 \text { ) }\end{array}$} & $\mathrm{C} 8 \mathrm{Cw}$ & $02 / 28 / 17$ & $0.5^{\mathrm{a}}$ & $M$ & Red Poll (Beef) & Severe \\
\hline & $\mathrm{C} 9 \mathrm{Cw}$ & $02 / 28 / 17$ & 15 & $\mathrm{~F}$ & Holstein (Dairy) & Moderate \\
\hline & $\mathrm{C} 10 \mathrm{CW}$ & $02 / 28 / 17$ & 8 & $\mathrm{~F}$ & Holstein mixed with Jamaica Hope (Dairy) & None \\
\hline & $\mathrm{C} 11 \mathrm{CW}$ & $02 / 28 / 17$ & 8 & $\mathrm{~F}$ & Jamaica Hope (Dairy) & None \\
\hline & $\mathrm{C} 12 \mathrm{Cw}$ & $02 / 28 / 17$ & 3 & $\mathrm{~F}$ & Jamaica Hope (Dairy) & None \\
\hline & $\mathrm{C} 13 \mathrm{Cw}$ & $02 / 28 / 17$ & 4 & $\mathrm{~F}$ & Jamaica Hope (Dairy) & None \\
\hline & $\mathrm{C} 14 \mathrm{Cw}$ & $02 / 28 / 17$ & 3 & $\mathrm{~F}$ & Jamaica Hope (Dairy) & None \\
\hline & $\mathrm{C} 15 \mathrm{CW}$ & $02 / 28 / 17$ & 3 & $\mathrm{~F}$ & Jamaica Hope (Dairy) & None \\
\hline & $46 \mathrm{BeCw}$ & $03 / 13 / 17$ & 3 & M & Red Poll (Beef) & None \\
\hline & $47 \mathrm{BeCw}$ & $03 / 13 / 17$ & 1.5 & $\mathrm{M}$ & Indian (Beef) & None \\
\hline & $48 \mathrm{CW}$ & $03 / 13 / 17$ & $0.25^{\mathrm{a}}$ & $M$ & Black Poll (Beef) & None \\
\hline & $49 \mathrm{BeCw}$ & $03 / 13 / 17$ & 4 & $\mathrm{~F}$ & Indian mixed with common (Beef) & Moderate \\
\hline
\end{tabular}




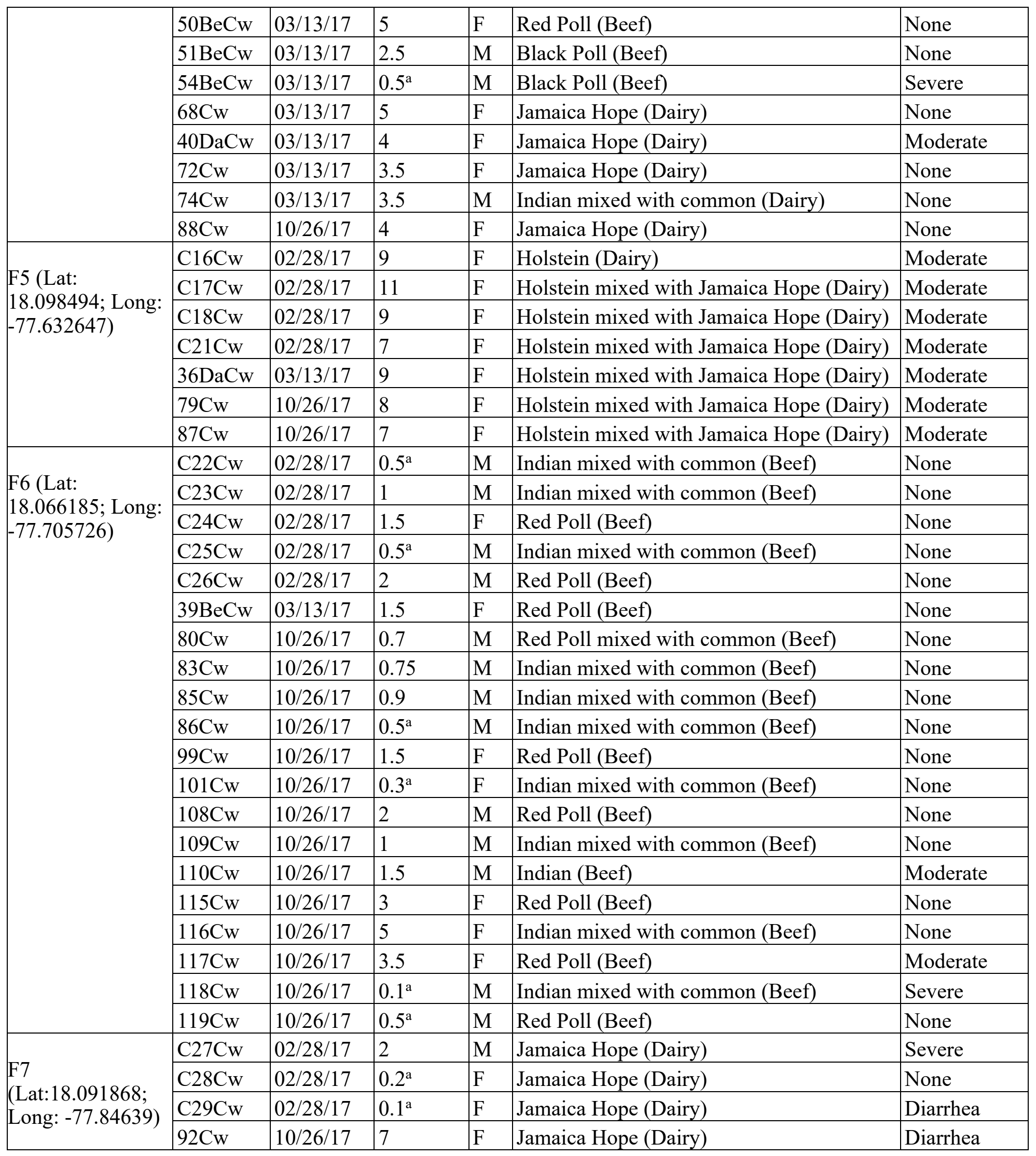




\begin{tabular}{|c|c|c|c|c|c|c|}
\hline & $104 \mathrm{CW}$ & $10 / 26 / 17$ & $0.1^{\mathrm{a}}$ & $\mathrm{F}$ & Jamaica Hope (Dairy) & Diarrhea \\
\hline & $52 \mathrm{DaCw}$ & $03 / 13 / 17$ & 1.5 & $\mathrm{~F}$ & Jamaica Hope (Dairy) & None \\
\hline & $53 \mathrm{DaCw}$ & 03/13/17 & $0.2^{\mathrm{a}}$ & $\mathrm{F}$ & Jamaica Hope (Dairy) & Diarrhea \\
\hline & $55 \mathrm{DaCw}$ & $03 / 13 / 17$ & 5 & $\mathrm{~F}$ & Jamaica Hope (Dairy) & Diarrhea \\
\hline & $56 \mathrm{DaCw}$ & $03 / 13 / 17$ & 1 & $\mathrm{~F}$ & Jamaica Hope (Dairy) & Diarrhea \\
\hline & $57 \mathrm{DaCw}$ & $03 / 13 / 17$ & 3.5 & $\mathrm{~F}$ & Jamaica Hope (Dairy) & None \\
\hline & $58 \mathrm{DaCw}$ & $03 / 13 / 17$ & $0.5^{\mathrm{a}}$ & $\mathrm{F}$ & Jamaica Hope (Dairy) & Diarrhea \\
\hline & $59 \mathrm{DaCw}$ & $03 / 13 / 17$ & 5 & $\mathrm{~F}$ & Jamaica Hope (Dairy) & None \\
\hline & $60 \mathrm{DaCw}$ & $03 / 13 / 17$ & 3 & $\mathrm{~F}$ & Jamaica Hope (Dairy) & None \\
\hline & $61 \mathrm{DaCw}$ & $03 / 13 / 17$ & 2 & $\mathrm{~F}$ & Jamaica Hope (Dairy) & None \\
\hline & $95 \mathrm{Cw}$ & $10 / 26 / 17$ & 2 & $\mathrm{M}$ & Jamaica Hope (Dairy) & None \\
\hline & $73 \mathrm{Cw}$ & $03 / 13 / 17$ & $0.2^{\mathrm{a}}$ & $\mathrm{F}$ & Jamaica Hope (Dairy) & None \\
\hline & $89 \mathrm{CW}$ & $10 / 26 / 17$ & 0.7 & $\mathrm{~F}$ & Jamaica Hope (Dairy) & None \\
\hline & $90 \mathrm{CW}$ & $10 / 26 / 17$ & 3 & $\mathrm{~F}$ & Jamaica Hope (Dairy) & None \\
\hline \multirow{6}{*}{$\begin{array}{l}\text { F8 (Lat: } \\
\text { 18.128619; Long: } \\
-77.649231 \text { ) }\end{array}$} & $\mathrm{C} 30 \mathrm{Cw}$ & $02 / 28 / 17$ & $0.5^{\mathrm{a}}$ & $\mathrm{F}$ & Indian (Beef) & None \\
\hline & $\mathrm{C} 31 \mathrm{Cw}$ & $02 / 28 / 17$ & 1.5 & F & Black Poll (Beef) & None \\
\hline & $113 \mathrm{Cw}$ & $02 / 28 / 17$ & 3 & $\mathrm{~F}$ & Indian (Beef) & Diarrhea \\
\hline & $70 \mathrm{CW}$ & $03 / 13 / 17$ & 4 & $\mathrm{~F}$ & Indian mixed with common (Beef) & None \\
\hline & $71 \mathrm{CW}$ & $03 / 13 / 17$ & 2 & $\mathrm{~F}$ & Black Poll (Beef) & None \\
\hline & $82 \mathrm{CW}$ & $10 / 26 / 17$ & $0.5^{\mathrm{a}}$ & $\mathrm{M}$ & Brahman mixed with common (Beef) & None \\
\hline \multirow{19}{*}{$\begin{array}{l}\text { F9 (Lat: } \\
\text { 18.125799; Long: } \\
-77.656546 \text { ) }\end{array}$} & $\mathrm{C} 32 \mathrm{Cw}$ & $03 / 13 / 17$ & 1 & $\mathrm{~F}$ & Red Poll mixed with common (Beef) & Diarrhea \\
\hline & $35 \mathrm{DaCw}$ & $03 / 13 / 17$ & $0.5^{\mathrm{a}}$ & $\mathrm{M}$ & Jamaica Hope (Dairy) & None \\
\hline & $34 \mathrm{DaCw}$ & $03 / 13 / 17$ & 2 & $\mathrm{~F}$ & Holstein (Dairy) & Diarrhea \\
\hline & $69 \mathrm{Cw}$ & $03 / 13 / 17$ & 1.5 & $\mathrm{~F}$ & Red Poll (Beef) & None \\
\hline & $84 \mathrm{Cw}$ & $03 / 13 / 17$ & 5 & $\mathrm{~F}$ & Red Poll (Beef) & None \\
\hline & $38 \mathrm{BeCw}$ & $03 / 13 / 17$ & 4 & $\mathrm{~F}$ & Indian mixed with common (Beef) & None \\
\hline & $97 \mathrm{Cw}$ & $10 / 26 / 17$ & 2.5 & $\mathrm{~F}$ & Holstein (Dairy) & Diarrhea \\
\hline & $41 \mathrm{DaCw}$ & $03 / 13 / 17$ & 1.5 & $\mathrm{~F}$ & Jamaica Hope (Dairy) & None \\
\hline & $42 \mathrm{DaCw}$ & $03 / 13 / 17$ & 6 & $M$ & Holstein (Dairy) & None \\
\hline & $43 \mathrm{DaCw}$ & $03 / 13 / 17$ & 2.5 & $\mathrm{~F}$ & Holstein (Dairy) & Diarrhea \\
\hline & $44 \mathrm{DaCw}$ & $03 / 13 / 17$ & 2 & $\mathrm{~F}$ & Jamaica Hope (Dairy) & None \\
\hline & $45 \mathrm{DaCw}$ & $03 / 13 / 17$ & 1.5 & $\mathrm{~F}$ & Jamaica Hope (Dairy) & None \\
\hline & $37 \mathrm{BeCw}$ & $03 / 13 / 17$ & 4 & $\mathrm{M}$ & Black Poll (Beef) & None \\
\hline & $81 \mathrm{Cw}$ & $10 / 26 / 17$ & 1 & $\mathrm{~F}$ & Red Poll mixed with common (Beef) & Diarrhea \\
\hline & $76 \mathrm{CW}$ & $03 / 13 / 17$ & $0.25^{\mathrm{a}}$ & $\mathrm{F}$ & Jamaica Hope (Dairy) & None \\
\hline & $78 \mathrm{CW}$ & $03 / 13 / 17$ & 1.5 & $\mathrm{~F}$ & Jamaica Hope (Dairy) & None \\
\hline & $62 \mathrm{DaCw}$ & $03 / 13 / 17$ & 2 & $\mathrm{~F}$ & Holstein (Dairy) & None \\
\hline & $63 \mathrm{DaCw}$ & $03 / 13 / 17$ & 2 & $\mathrm{~F}$ & Jamaica Hope (Dairy) & None \\
\hline & 64BeCw & $03 / 13 / 17$ & 3 & $\mathrm{M}$ & Brahman (Beef) & Diarrhea \\
\hline
\end{tabular}




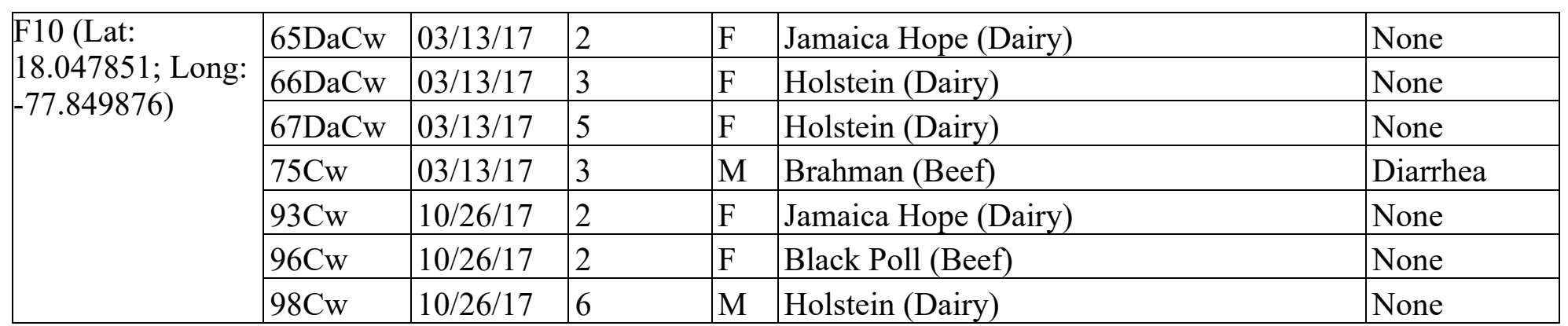

${ }^{\text {a }}$ Pre-weaned calf; 0 to $0.5 \mathrm{yr}(n=23)$

0.6 to $1 \mathrm{yr}(n=26)$

2 to 3 yr $(n=37)$

4 to $5 \mathrm{yr}(n=17)$

$\geq 6 \operatorname{yr}(n=16)$

Table C.II.: MAF/ELISA/PCR Detections of Cryptosporidium in Cattle Feces According to Age, Production Type and Diarrheal Symptom

\begin{tabular}{|l|c|c|c|c|c|c|c|}
\hline Farm & Cow ID & MAF & ELISA & PCR & Age & $\begin{array}{c}\text { Production } \\
\text { Type }\end{array}$ & $\begin{array}{c}\text { Diarrheal } \\
\text { Symptoms }\end{array}$ \\
\hline F1 & C2Cw & + & & & 4 & Beef & None \\
\hline F1 & C5Cw & + & + & & 1 & Beef & None \\
\hline F1 & $\mathrm{C} 6 \mathrm{Cw}$ & + & + & & 2.5 & Beef & Moderate \\
\hline F3 & $105 \mathrm{Cw}$ & + & + & + & 1 & Dairy & Moderate \\
\hline F4 & $\mathrm{C} 8 \mathrm{Cw}$ & + & + & + & $0.5^{\mathrm{a}}$ & Beef & Severe \\
\hline F4 & $\mathrm{C} 11 \mathrm{Cw}$ & + & + & & 8 & Dairy & None \\
\hline F4 & $\mathrm{C} 13 \mathrm{Cw}$ & & + & + & 4 & Dairy & None \\
\hline F4 & $46 \mathrm{BeCw}$ & + & & + & 3 & Beef & None \\
\hline F4 & $51 \mathrm{BeCw}$ & + & & & 2.5 & Beef & None \\
\hline F4 & $68 \mathrm{Cw}$ & & + & & 5 & Dairy & None \\
\hline F4 & $72 \mathrm{Cw}$ & & + & & 3.5 & Dairy & None \\
\hline F4 & $74 \mathrm{Cw}$ & + & + & & 3.5 & Dairy & None \\
\hline F4 & $40 \mathrm{DaCw}$ & & + & + & 4 & Dairy & Moderate \\
\hline F4 & $88 \mathrm{Cw}$ & + & & & 4 & Dairy & None \\
\hline F5 & $\mathrm{C} 18 \mathrm{Cw}$ & + & & & 9 & Dairy & Moderate \\
\hline F5 & $79 \mathrm{Cw}$ & + & & & 8 & Dairy & Moderate \\
\hline F6 & $\mathrm{C} 24 \mathrm{Cw}$ & + & & & 1.5 & Beef & None \\
\hline F6 & $101 \mathrm{Cw}$ & & + & & $0.3^{\mathrm{a}}$ & Beef & None \\
\hline F6 & $116 \mathrm{Cw}$ & + & & & 5 & Beef & None \\
\hline F7 & $\mathrm{C} 28 \mathrm{Cw}$ & + & + & & $0.2^{\mathrm{a}}$ & Dairy & None \\
\hline F7 & $53 \mathrm{DaCw}$ & + & + & + & $0.2^{\mathrm{a}}$ & Dairy & Moderate \\
\hline F7 & $55 \mathrm{DaCw}$ & + & + & & 5 & Dairy & Moderate \\
\hline F7 & $58 \mathrm{DaCw}$ & + & + & & $0.5^{\mathrm{a}}$ & Dairy & Moderate \\
\hline F7 & $61 \mathrm{DaCw}$ & & + & & 2 & Dairy & None \\
\hline F7 & $73 \mathrm{Cw}$ & + & & & $0.2^{\mathrm{a}}$ & Dairy & None \\
\hline
\end{tabular}




\begin{tabular}{|l|c|c|c|c|c|c|c|}
\hline F7 & $89 \mathrm{Cw}$ & + & + & & 0.7 & Dairy & None \\
\hline F7 & $56 \mathrm{Da}$ & + & + & + & 1 & Dairy & Moderate \\
\hline F7 & $60 \mathrm{Da}$ & + & & & 3 & Dairy & None \\
\hline F7 & $95 \mathrm{Cw}$ & + & & & 2 & Dairy & None \\
\hline F8 & $\mathrm{C} 31 \mathrm{Cw}$ & + & + & & 1.5 & Beef & None \\
\hline F8 & $70 \mathrm{Cw}$ & + & & & 4 & Beef & None \\
\hline F9 & $34 \mathrm{DaCw}$ & + & + & + & 2 & Dairy & Moderate \\
\hline F9 & $38 \mathrm{BeCw}$ & & + & & 4 & Beef & None \\
\hline F9 & $42 \mathrm{DaCw}$ & + & & & 6 & Dairy & None \\
\hline F9 & $78 \mathrm{Cw}$ & & + & + & 1.5 & Dairy & None \\
\hline F10 & $64 \mathrm{BeCw}$ & + & & + & 3 & Beef & Moderate \\
\hline F10 & $65 \mathrm{DaCw}$ & + & & & 2 & Dairy & None \\
\hline F10 & $75 \mathrm{Cw}$ & & + & & 3 & Beef & Moderate \\
\hline F10 & $98 \mathrm{Cw}$ & & + & & 6 & Dairy & None \\
\hline Total & - & $\mathbf{2 9}$ & $\mathbf{2 4}$ & $\mathbf{1 0}$ & - & - & - \\
Positive & & & & & & & \\
\hline
\end{tabular}

${ }^{a}$ Pre-weaned calf

Table C.III.: Cattle Population Density per Hectare of Land in St. Elizabeth as of October 2017

\begin{tabular}{|l|l|l|l|l|}
\hline District & \multicolumn{2}{|c|}{ Beef } & \multicolumn{2}{c|}{ Dairy } \\
\hline & Hectare of Land & Count & Hectare of Land & Count \\
\hline Balaclava & 519.7 & 1167 & 85.4 & 276 \\
\hline Black River & 2877.56 & 3468 & 420.16 & 987 \\
\hline Braes River & 1214.6 & 1890 & 114.19 & 550 \\
\hline Ginger Hill & 1319.8 & 1067 & 8.69 & 22 \\
\hline Ipswich & 358.96 & 277 & 0 & 0 \\
\hline Junction & 329.96 & 466 & 18 & 38 \\
\hline Lacovia & 1065.96 & 1511 & 6.74 & 50 \\
\hline Malvern & 725.08 & 921 & 73.23 & 202 \\
\hline Mountainside & 2064.77 & 4188 & 99.44 & 395 \\
\hline New Market & 634.46 & 891 & 28.93 & 21 \\
\hline Pedro Plains & 660.35 & 801 & 40.33 & 152 \\
\hline Santa Cruz & 1339.41 & 2580 & 30.84 & 70 \\
\hline Southfield & 110.67 & 241 & 3.84 & 5 \\
\hline Total & $\mathbf{1 3 2 2 1 . 2 8}$ & $\mathbf{1 9 4 6 8}$ & $\mathbf{9 2 9 . 7 3}$ & $\mathbf{2 7 6 8}$ \\
\hline
\end{tabular}

Note: Data set provide by Jamaica RADA (2017) 
Table C.IV.: Farm Management Data

\begin{tabular}{|c|c|c|c|c|c|}
\hline $\begin{array}{l}\text { Farm } \\
\text { I.D. }\end{array}$ & Production & $\begin{array}{l}\text { Drinking } \\
\text { Water } \\
\text { Source(s) }\end{array}$ & $\begin{array}{l}\text { Land Rearing/ } \\
\text { Flooring System }\end{array}$ & $\begin{array}{l}\text { Hygiene } \\
\text { Maintenance of Pen } \\
\text { or Land }\end{array}$ & Cow-calf Separation \\
\hline F1 & - beef & $\begin{array}{l}\text { - } \text { pipe }^{\text {a }} \\
\text { surface } \\
\text { water, } \\
\text { and rain }\end{array}$ & $\begin{array}{l}- \text { grass rearing } \\
-\quad \text { no fencing }\end{array}$ & $\begin{array}{ll}- & \text { feces cleared } \\
& \text { from land } 1-2 \\
& \text { times per week }\end{array}$ & $\begin{array}{l}\text { - no; cows are tied to } \\
\text { tree/peg with rope } \\
\text { and pre-weaned } \\
\text { calves roam freely }\end{array}$ \\
\hline F2 & - beef & $\begin{array}{l}- \text { pipe }^{\mathrm{a}} \\
\text { and rain }\end{array}$ & $\begin{array}{l}- \text { grass rearing } \\
-\quad \text { fencing }\end{array}$ & - none & $\begin{array}{l}-\quad \text { no; cows and calves } \\
\text { are freely roaming }\end{array}$ \\
\hline F3 & $\begin{array}{cl}- & \text { beef and } \\
& \text { dairy } \\
& \text { (raised } \\
& \text { separate) }\end{array}$ & $\begin{array}{l}- \text { pipe }^{\mathrm{a}} \\
\text { and rain }\end{array}$ & $\begin{array}{l}-\quad \text { concrete and } \\
\text { grass rearing } \\
-\quad \text { fencing }\end{array}$ & $\begin{array}{ll}- & \text { feces cleared } \\
\text { from land daily } \\
\text { - } \\
\text { concrete pen is } \\
\text { washed daily } \\
\text { with water and } \\
\text { occasionally } \\
\text { with } \\
\text { disinfectant }\end{array}$ & $\begin{array}{l}\text { yes; cows freely } \\
\text { roam on grass and } \\
\text { pre-weaned calves } \\
\text { are raised on } \\
\text { concreate }\end{array}$ \\
\hline F4 & $\begin{array}{cl}- & \text { beef and } \\
& \text { dairy } \\
\text { (raised } & \\
& \text { separate) }\end{array}$ & $\begin{array}{ll} & \text { pipe }^{\mathrm{a}} \\
\text { surface } \\
\text { water } \\
\text { (beef), } \\
\text { and rain }\end{array}$ & $\begin{array}{ll}- & \text { concrete/dirt } \\
& \text { and grass } \\
& \text { rearing } \\
- & \text { partial } \\
& \text { fencing }\end{array}$ & $\begin{array}{ll}- & \text { feces cleared } \\
\text { from land 2-3 } \\
\text { times per week } \\
\text { - } & \text { concrete pen is } \\
\text { washed daily } \\
\text { with water and } \\
\text { weekly with } \\
\text { disinfectant }\end{array}$ & $\begin{array}{l}- \text { yes; cows freely } \\
\text { roam on grass and } \\
\text { pre-weaned calves } \\
\text { are raised on } \\
\text { concreate/dirt }\end{array}$ \\
\hline F5 & - dairy & $\begin{array}{l}- \text { pipe }^{\mathrm{a}} \\
\text { and rain }\end{array}$ & $\begin{array}{ll}- & \text { grass and } \\
\text { gravel } \\
\text { rearing } \\
-\quad \text { fencing }\end{array}$ & $\begin{array}{l}-\quad \text { feces cleared } \\
\text { from pen } \\
\text { multiple times } \\
\text { per day }\end{array}$ & $\begin{array}{l}\text { - yes; freely roaming, } \\
\text { but adult cows are } \\
\text { separated from } \\
\text { calves }\end{array}$ \\
\hline F6 & - beef & $\begin{array}{l}- \text { pipe }^{\mathrm{a}} \\
\text { and rain }\end{array}$ & $\begin{array}{ll}- & \text { grass rearing } \\
- & \text { partial } \\
& \text { fencing }\end{array}$ & - none & $\begin{array}{l}- \text { no; cows are tied to } \\
\text { tree/peg with rope } \\
\text { and pre-weaned } \\
\text { calves roam freely }\end{array}$ \\
\hline F7 & - dairy & $\begin{array}{l}- \text { pipe }^{\mathrm{a}} \\
\text { and rain }\end{array}$ & $\begin{array}{l}- \text { grass rearing } \\
-\quad \text { fencing }\end{array}$ & $\begin{array}{ll}- & \text { feces are } \\
\text { cleared } \\
\text { multiple times } \\
\text { per day }\end{array}$ & $\begin{array}{l}\text { yes; freely roaming, } \\
\text { but pre-weaned, } \\
\text { young and adult } \\
\text { cows are separated }\end{array}$ \\
\hline
\end{tabular}




\begin{tabular}{|c|c|c|c|c|c|}
\hline F8 & $-\quad$ beef & $\begin{array}{l}- \text { pipe }^{\mathrm{a}} \\
\text { and rain }\end{array}$ & $\begin{array}{ll}- & \text { concrete and } \\
\text { grass rearing } \\
- & \text { fencing }\end{array}$ & - none & - no \\
\hline F9 & $\begin{array}{ll}- & \text { beef and } \\
& \text { dairy } \\
\text { (raised } & \\
\text { separate) }\end{array}$ & $\begin{array}{l}- \text { pipe }^{\mathrm{a}} \\
\text { and rain }\end{array}$ & $\begin{array}{ll}- & \text { concrete and } \\
\text { grass rearing } \\
- & \text { fencing }\end{array}$ & $\begin{array}{l}-\quad \text { feces are not } \\
\text { cleared from } \\
\text { grassland } \\
-\quad \text { concrete pen is } \\
\text { washed daily } \\
\text { with disinfect }\end{array}$ & - no \\
\hline F10 & $\begin{array}{ll}- & \text { beef and } \\
\text { dairy } \\
\text { (raised } \\
\text { together) }\end{array}$ & $\begin{array}{l}\text { - } \text { surface } \\
\text { water }^{\mathrm{a}}, \\
\text { and rain }\end{array}$ & $\begin{array}{ll}- & \text { grass and } \\
& \text { gravel } \\
& \text { rearing } \\
- & \text { fencing }\end{array}$ & - none & $\begin{array}{l}-\quad \text { no; cows and calves } \\
\text { roam freely in } \\
\text { enclosed area }\end{array}$ \\
\hline
\end{tabular}

${ }^{a}$ primary drinking water source

Table C.V.: COMSTAT2 Analyses of Biofilm Segments

\begin{tabular}{|c|c|c|c|c|}
\hline $\begin{array}{l}\text { Biofilm } \\
\text { I.D. }\end{array}$ & $\begin{array}{l}\text { Replicate Biofilm } \\
\text { Segments }\end{array}$ & $\begin{array}{l}\text { Average Biofilm } \\
\text { Thickness }\end{array}$ & $\begin{array}{l}\text { Maximum } \\
\text { Biofilm } \\
\text { Thickness } \\
\end{array}$ & $\begin{array}{l}\text { Roughness } \\
\text { Coefficient }\end{array}$ \\
\hline \multirow{8}{*}{ Bply1 } & $\mathrm{R} 1$ & \begin{tabular}{|l}
34.87 \\
\end{tabular} & 62 & 1.87 \\
\hline & R2 & 20.43 & 86 & 1.96 \\
\hline & R3 & 6.21 & 52 & 1.98 \\
\hline & R4 & 68.84 & 126 & 1.52 \\
\hline & R5 & 33.61 & 94 & 1.85 \\
\hline & R6 & 49.47 & 112 & 1.7 \\
\hline & R7 & 69 & 111 & 1.35 \\
\hline & R8 & 104.2 & 132 & 1.88 \\
\hline \multirow{8}{*}{ Bply2 } & R1 & 34.98 & 108 & 1.82 \\
\hline & R2 & 36.1 & 87 & 1.9 \\
\hline & R3 & \begin{tabular}{|l}
10.72 \\
\end{tabular} & 35 & 1.26 \\
\hline & R4 & 83.34 & 116 & 1.86 \\
\hline & R5 & 66.4 & 104 & 2.01 \\
\hline & R6 & 82.14 & 113 & 1.77 \\
\hline & R7 & 25.23 & 98 & 1.36 \\
\hline & R8 & 83.3 & 104 & 1.89 \\
\hline \multirow{6}{*}{ Bply3 } & R1 & \begin{tabular}{|l}
101.35 \\
\end{tabular} & 128 & 1.66 \\
\hline & R2 & 68.46 & 111 & 1.38 \\
\hline & R3 & 57.36 & 97 & 1.89 \\
\hline & R4 & 87.2 & 131 & 1.78 \\
\hline & R5 & 98.21 & 109 & 2.13 \\
\hline & R6 & 71.58 & 92 & 2.19 \\
\hline
\end{tabular}




\begin{tabular}{|c|c|c|c|c|}
\hline & R7 & 109.36 & 136 & 1.79 \\
\hline & R8 & 66.71 & 117 & 1.51 \\
\hline \multirow{8}{*}{ Bply4 } & $\mathrm{R} 1$ & 35.4 & 94 & 1.64 \\
\hline & R2 & 101.6 & 113 & 1.39 \\
\hline & R3 & 52.69 & 123 & 1.44 \\
\hline & R4 & 82.5 & 105 & 1.72 \\
\hline & R5 & 23.6 & 88 & 1.8 \\
\hline & R6 & 10.4 & 62 & 1.92 \\
\hline & $\mathrm{R} 7$ & 80.3 & 104 & 1.83 \\
\hline & R8 & 97.23 & 114 & 1.36 \\
\hline Mean & - & 61.02 & 102 & 1.73 \\
\hline S.D. & - & $\mathbf{3 0 . 3 7}$ & 22.98 & 0.24 \\
\hline \multirow{8}{*}{ BglsREF } & $\mathrm{R} 1$ & 23.36 & 32.64 & 0.21 \\
\hline & R2 & 4.32 & 17.98 & 0.64 \\
\hline & R3 & 17.06 & 36.05 & 0.36 \\
\hline & R4 & 28.14 & 30.15 & 0.31 \\
\hline & R5 & 26.35 & 43.21 & 0.48 \\
\hline & R6 & 11.34 & 39.35 & 0.13 \\
\hline & R7 & 19.43 & 26.25 & 0.42 \\
\hline & R8 & 7.68 & 16.28 & 0.44 \\
\hline Mean & - & 17.21 & 30.24 & 0.37 \\
\hline S.D. & - & 8.19 & 9.03 & 0.15 \\
\hline
\end{tabular}

Table C.VI.: Recovery of $C$. Parvum Oocysts from Biofilm Suspensions by IMS

\begin{tabular}{|c|c|c|c|c|}
\hline Biofilm I.D. & $\begin{array}{l}\text { Replicate } \\
\text { Microscope Slide }\end{array}$ & $\begin{array}{l}\text { No. of oocysts Counted } \\
\text { in Columns }\end{array}$ & $\begin{array}{l}\text { Calculated Recovery } \\
(\%)\end{array}$ & $\begin{array}{l}\text { Mean Turbidity } \\
\text { (NTU) }^{\mathrm{a}}\end{array}$ \\
\hline \multirow{4}{*}{ Bply1 } & $\mathrm{R} 1$ & 378 & 63.58 & \multirow{4}{*}{$544.1 \pm 24.72$} \\
\hline & $\mathrm{R} 2$ & 459 & 77.21 & \\
\hline & $\mathrm{R} 3$ & 403 & 67.79 & \\
\hline & $\mathrm{R} 4$ & 394 & 66.27 & \\
\hline \multirow{4}{*}{ Bply2 } & $\mathrm{R} 1$ & 214 & 35.1 & \multirow{4}{*}{$554.1 \pm 26.81$} \\
\hline & $\mathrm{R} 2$ & 227 & 38.18 & \\
\hline & $\mathrm{R} 3$ & 183 & 30.78 & \\
\hline & $\mathrm{R} 4$ & 211 & 35.5 & \\
\hline \multirow{4}{*}{ Bply3 } & $\mathrm{R} 1$ & 223 & 37.51 & \multirow{4}{*}{$549.6 \pm 23.64$} \\
\hline & $\mathrm{R} 2$ & 408 & 68.63 & \\
\hline & R3 & 218 & 36.67 & \\
\hline & $\mathrm{R} 4$ & 195 & 32.8 & \\
\hline
\end{tabular}




\begin{tabular}{|l|l|l|l|l|}
\hline \multirow{5}{*}{} & R1 & 189 & 31.79 & \multirow{5}{*}{$544.3 \pm 27.14$} \\
\cline { 2 - 4 } & R2 & 136 & 22.88 & \\
\cline { 2 - 4 } R3 & 147 & 24.73 & \\
\cline { 2 - 4 } Bply4 & R4 & 96 & 16.15 & $\mathbf{5 4 8 . 1}$ \\
\hline S.D. & - & $\mathbf{2 4 6 . 8 7}$ & $\mathbf{4 2 . 9}$ & $\mathbf{2 5 . 9 5}$ \\
\hline
\end{tabular}

${ }^{\mathrm{a}}$ Mean \pm S.D. $(n=10)$

\section{Table C.VII.: Spectrophotometric (NanoDrop ND1000) Readings}

Sample ID
C8Cw
C17Cw
D3Bio
D2Bio

CPB-DIAG F (100)

CPB-DIAG R (100)

dil CPB-DIAG F (5)

dil CPB-DIAG R (5)

DNA ctrl

NFW

\section{Sample ID}

KLJ F (100)

KLJ F (100)

dil KLJ F (5)

dil KLJ R (5)

DNA ctrl

NFW
User ID Date

Default

Default $10 / 05 / 2017$

Default 10/05/2017

Default

Default

Default
$10 / 05 / 2017$

$10 / 05 / 2017$

$10 / 05 / 2017$
Time

2:14 PM

12:15 PM

12:26 PM

12:27 PM

12:22 PM

12:22 PM

12:22 PM

12:22 PM

$12: 18$ PM

12:28 PM ng/ul A260

10.57

0.630 .013

267.13

319.11

642.31

925.65

35.67

42.1

90.3

1.54

A280 260

$0.08 \quad 2.65$

$0.02 \quad 0.63$

2.809

3.371

12.71

13.61

0.712

0.831

0.943

$-0.02$

$60 / 280$
2.65
0.63
1.9
1.89
1.53
2.06
1.52
1.54
1.92
-1.67

$260 / 230$
0.07
0.69
2.24
2.27
1.41
2.71
2.63
2.14
1.77
2.67

Cursor

Pos.

230

230

230

230

230

230

230

230

230

230
Cursor

abs.

3.224

0.018

2.385

2.809

13.792

12.58

0.468

0.435

1.018

0.012
340

raw

$-0.144$

$-0.002$

0.04

0.048

39.045

0.064

0.026

0.037

$-0.029$

$-0.001$

\begin{tabular}{|c|c|c|c|c|c|c|c|c|c|c|c|c|}
\hline Sample ID & User ID & Date & Time & $\mathrm{ng} / \mathrm{ul}$ & A260 & A280 & $260 / 280$ & $260 / 230$ & Constant & $\begin{array}{r}\text { Cursor } \\
\text { Pos. }\end{array}$ & $\begin{array}{r}\text { Cursor } \\
\text { abs. }\end{array}$ & $\begin{array}{l}340 \\
\text { raw }\end{array}$ \\
\hline XIAO 1F (100) & Default & $7 / 12 / 2018$ & 4:29 PM & 552.59 & 16.745 & 9.084 & 1.84 & 2.39 & 33 & 230 & 7.001 & 0.097 \\
\hline XIAO 1R (100) & Default & $7 / 12 / 2018$ & 4:30 PM & 596.4 & 18.073 & 10.613 & 1.7 & 2.1 & 33 & 230 & 8.614 & 0.062 \\
\hline XIAO $2 F(100)$ & Default & $7 / 12 / 2018$ & 4:30 PM & 736.67 & 22.323 & 10.835 & 2.06 & 2.93 & 33 & 230 & 7.627 & 0.152 \\
\hline
\end{tabular}




\begin{tabular}{|c|c|c|c|c|c|c|c|c|c|c|c|c|}
\hline XIAO 2R (100) & Default & $7 / 12 / 2018$ & 4:31 PM & 630.25 & 19.098 & 8.76 & 2.18 & 2.3 & 33 & 230 & 8.286 & 0.025 \\
\hline dil XIAO $1 F(5)$ & Default & $7 / 12 / 2018$ & 4:32 PM & 28.74 & 0.871 & 0.538 & 1.62 & 2.5 & 33 & 230 & 0.348 & 0.018 \\
\hline dil XIAO 1R (5) & Default & $7 / 12 / 2018$ & 4:32 PM & 30.05 & 0.911 & 0.61 & 1.49 & 2.22 & 33 & 230 & 0.409 & 0.024 \\
\hline dil XIAO 2F (5) & Default & $7 / 12 / 2018$ & 4:33 PM & 40.76 & 1.235 & 0.636 & 1.94 & 2.88 & 33 & 230 & 0.429 & 0.022 \\
\hline dil XIAO 2R (5) & Default & $7 / 12 / 2018$ & 4:33 PM & 34.18 & 1.036 & 0.543 & 1.91 & 2.32 & 33 & 230 & 0.446 & 0.059 \\
\hline NFW & Default & $7 / 12 / 2018$ & 4:33 PM & 0.87 & 0.026 & 0.062 & 0.42 & 0.83 & 33 & 230 & 0.031 & 0.02 \\
\hline LMP1 -1409 & Default & $7 / 12 / 2018$ & 4:35 PM & 11.03 & 0.221 & 0.123 & 1.79 & 2.05 & 50 & 230 & 0.108 & -0.03 \\
\hline NFW & Default & $7 / 12 / 2018$ & 4:35 PM & -0.56 & -0.011 & 0.001 & -9.8 & 0.57 & 50 & 230 & -0.02 & 0.006 \\
\hline
\end{tabular}



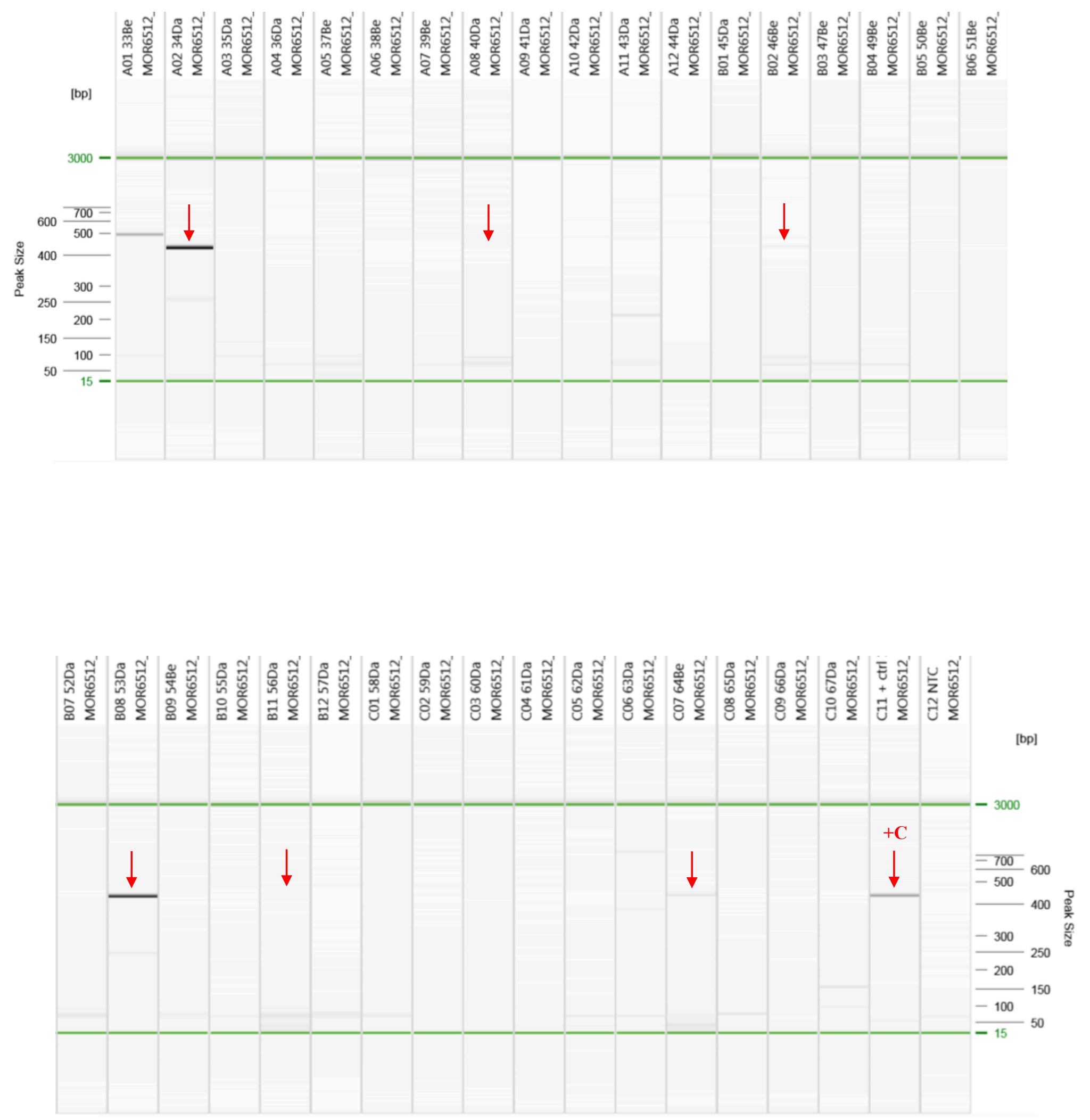

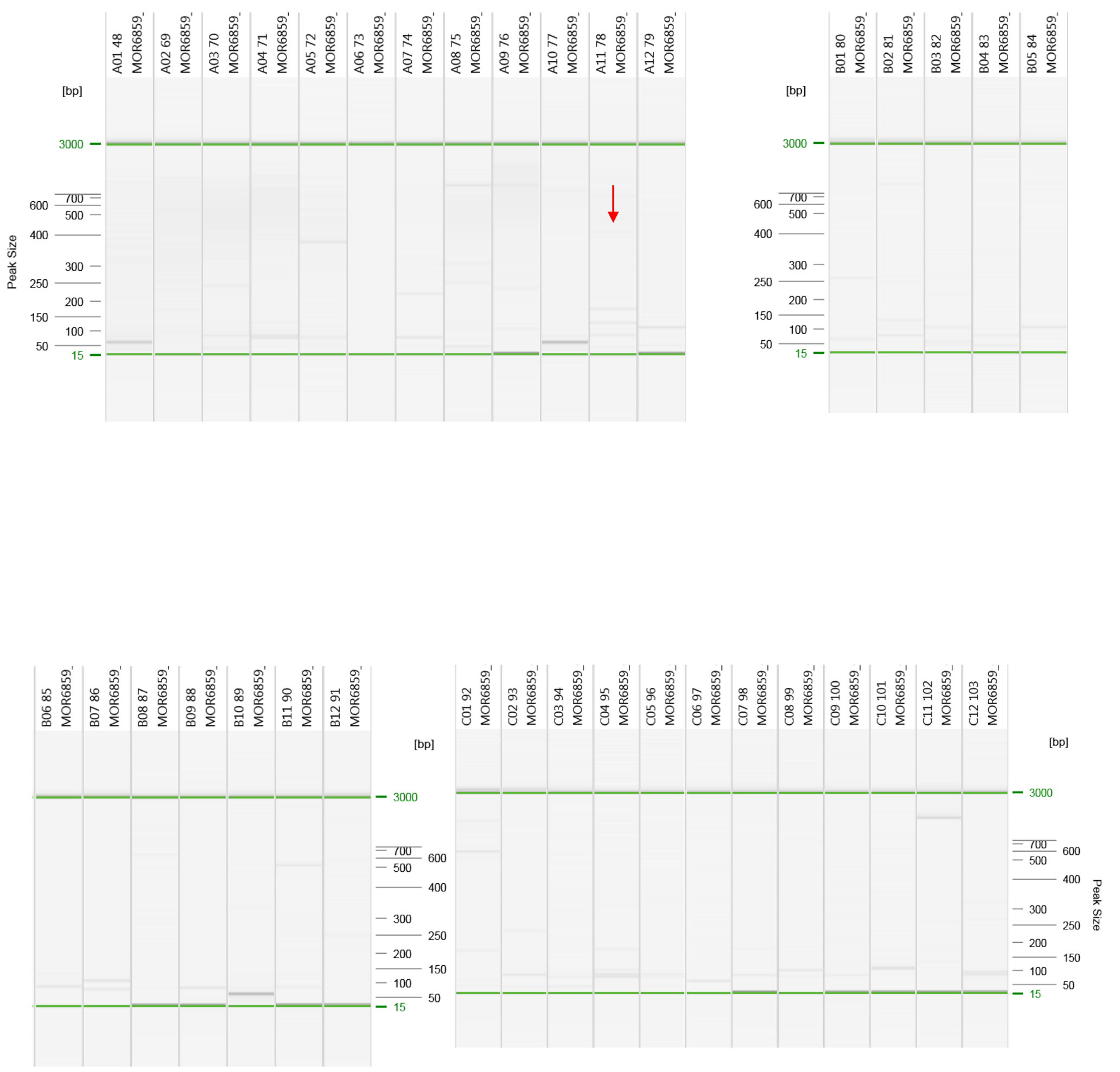

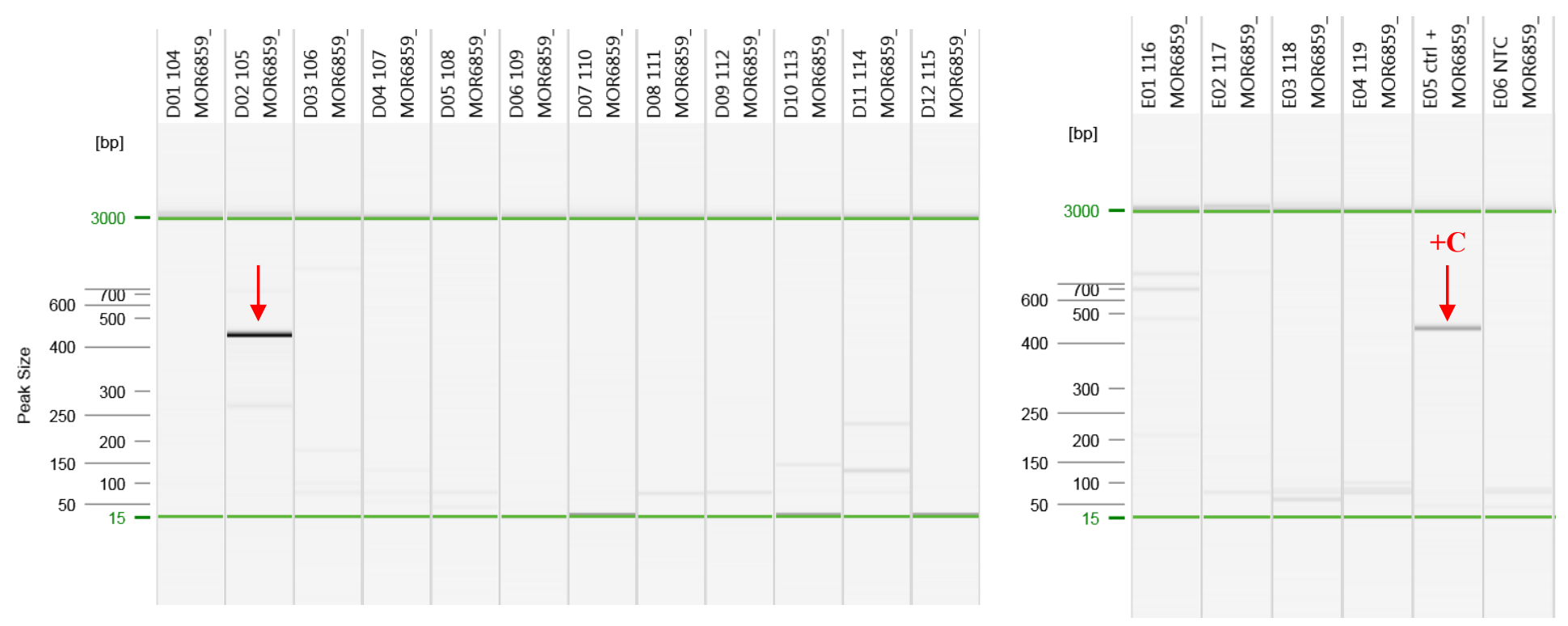

Figure D.I.: Electrophoresis images of Cryptosporidium detection derived from conventional PCR products targeting the 435 bp of the $18 S$ rRNA.

First Round

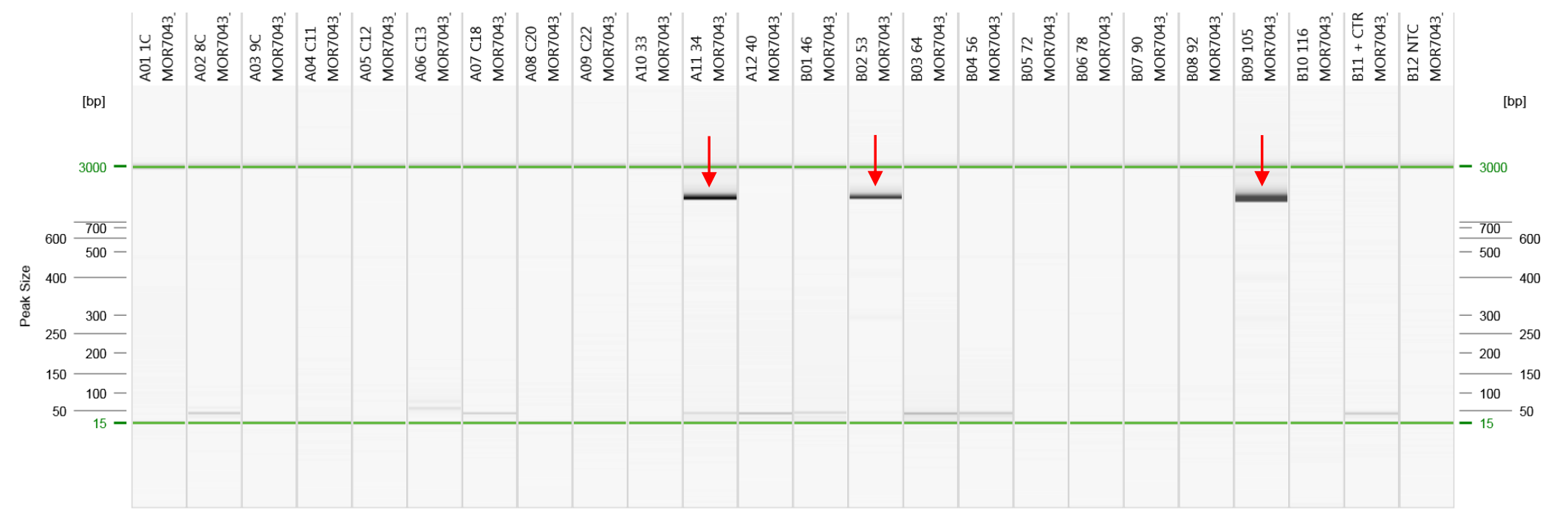


Second Round

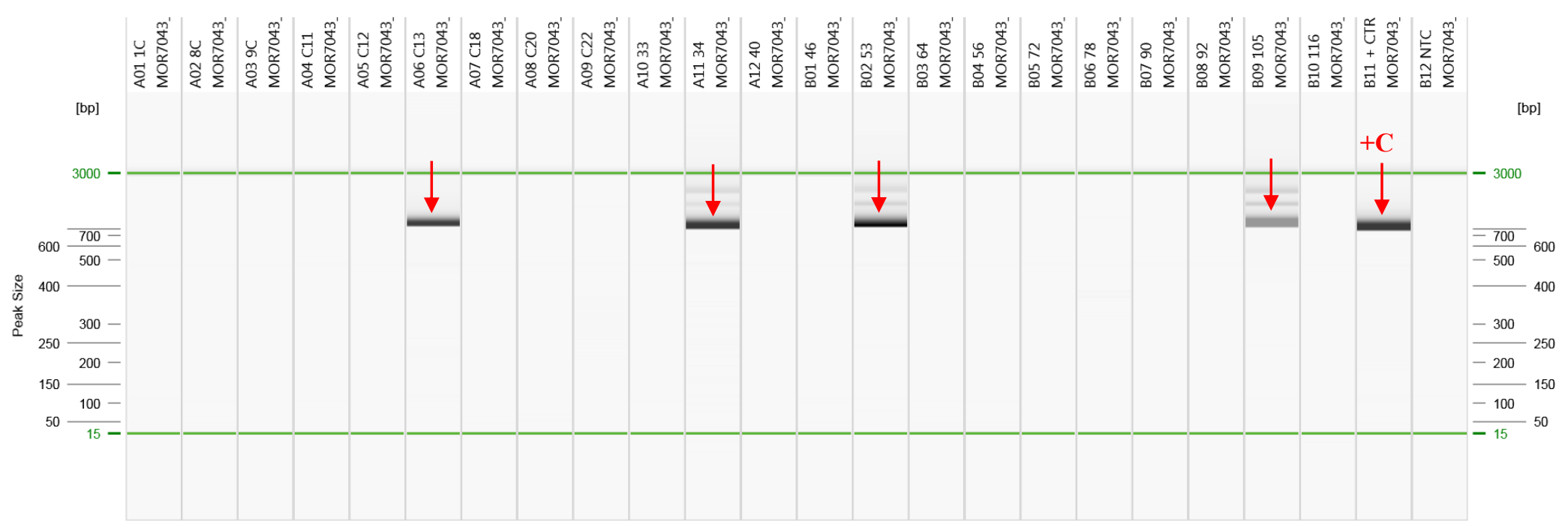

Figure D.II.: Electrophoresis images of Cryptosporidium detection derived from nestedPCR products targeting the 1325 bp (first round) and 819-835 bp (second round) of the 18S rRNA.
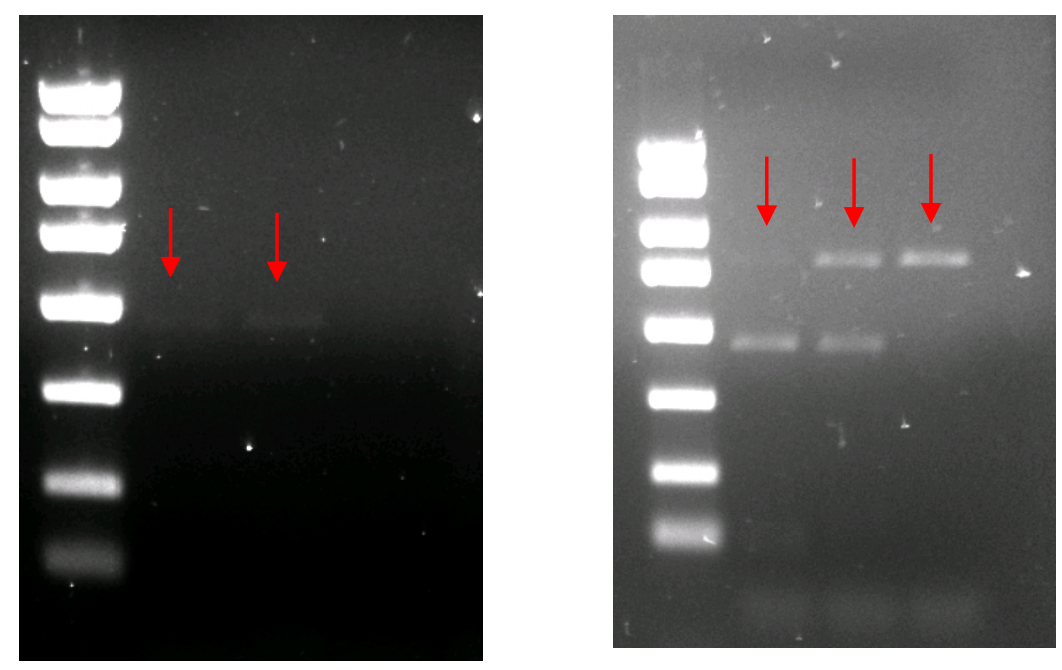

Figure D.III.: Electrophoresis images confirming DNA used for Cryptosporidium Positive Controls. PCR products targeting (a) $435 \mathrm{bp}$ and (b) $1325 \mathrm{bp}$ (first round) and 819-835 (second round) of $18 \mathrm{~S}$ rRNA. 


\section{Appendix E: Chromatogram Readings of 18S rRNA Multiple Sequence Alignments}

$\begin{array}{lllllllllllllllllllllllllllllllll}10 & 20 & 30 & 40 & 50 & 60 & 70 & 80 & 90 & 100 & 110 & 120 & 130 & 140 & 150 & 160 & 170 & 180 & 190 & 200 & 210 & 220 & 230 & 240 & 250 & 260 & 270 & 280 & 290 & 300 & 310 & 320 & 330\end{array}$

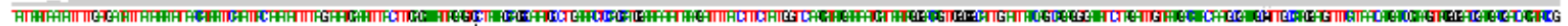

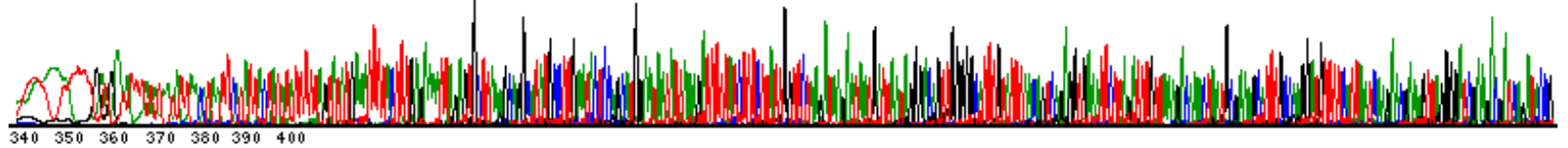

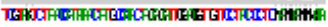

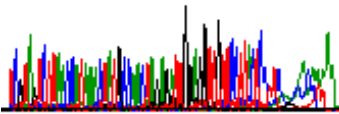

Biofilm Sample: C2Bio

$\begin{array}{llllllllllllllllllllllllllllllllllll}10 & 20 & 30 & 40 & 50 & 60 & 70 & 80 & 90 & 100 & 110 & 120 & 130 & 140 & 150 & 160 & 170 & 180 & 190 & 200 & 210 & 220 & 230 & 240 & 250 & 260 & 270 & 280 & 290 & 300 & 310 & 320 & 330\end{array}$

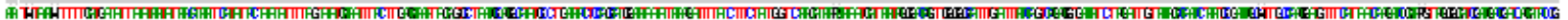

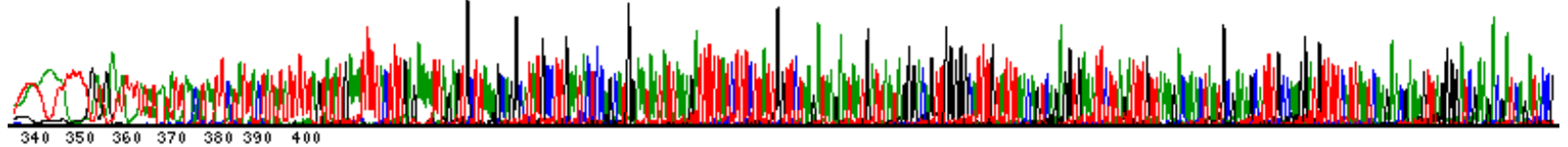

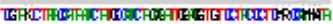

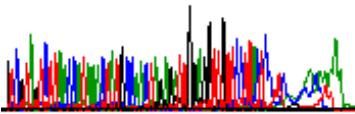

Biofilm Sample: D1Bio

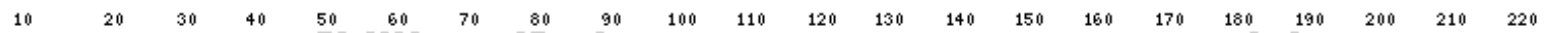

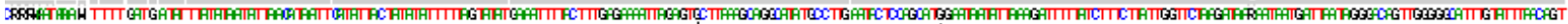

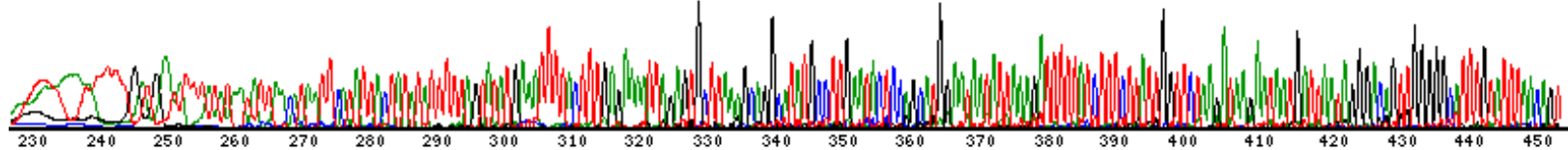

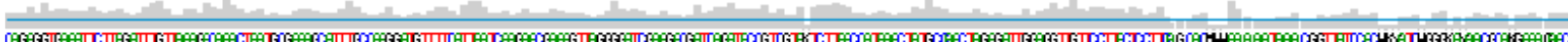

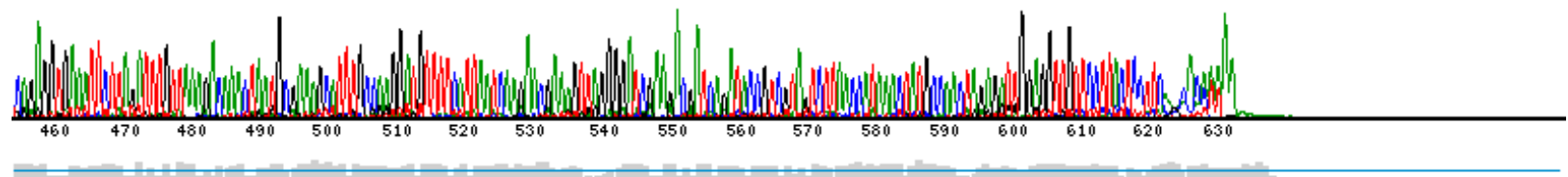

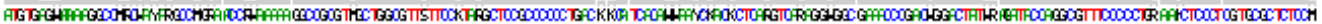

Biofilm Sample: D3Bio 


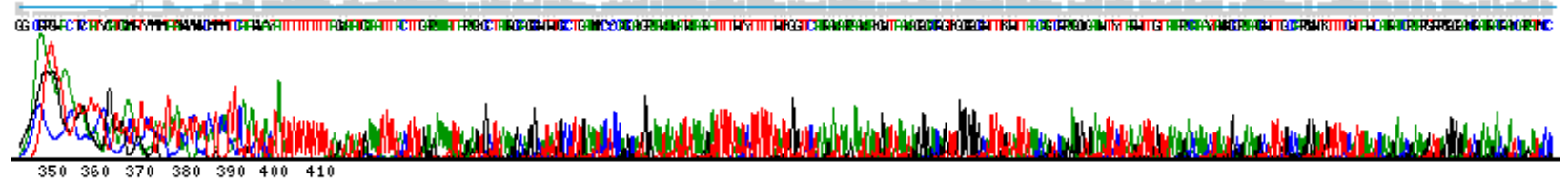

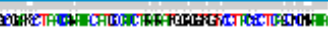

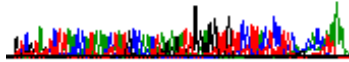

Cattle Sample: $\mathrm{C} 8 \mathrm{Cw}$

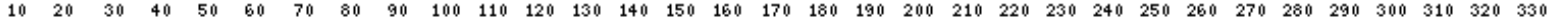

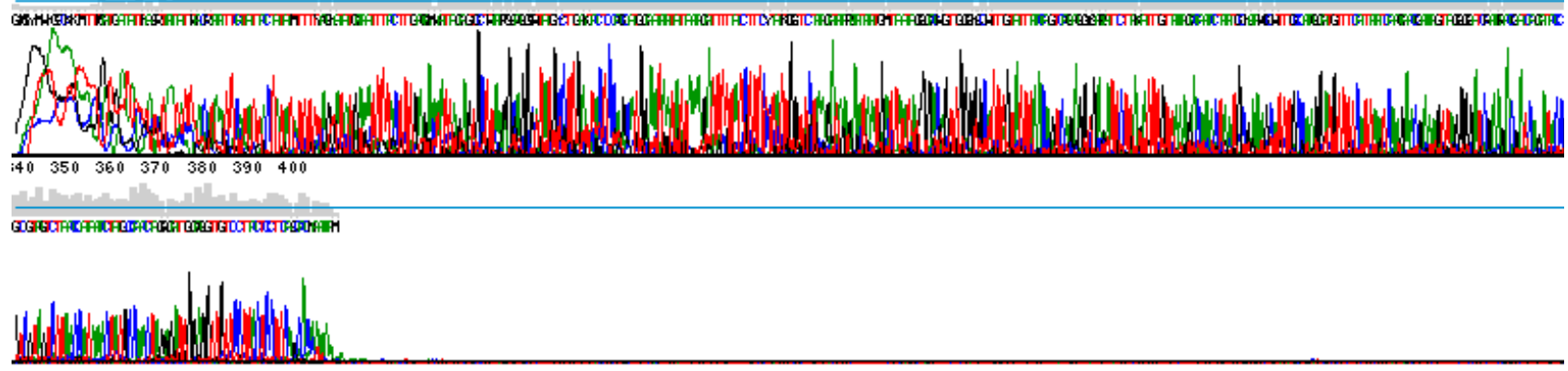

Cattle Sample: $34 \mathrm{DaCw}$ 


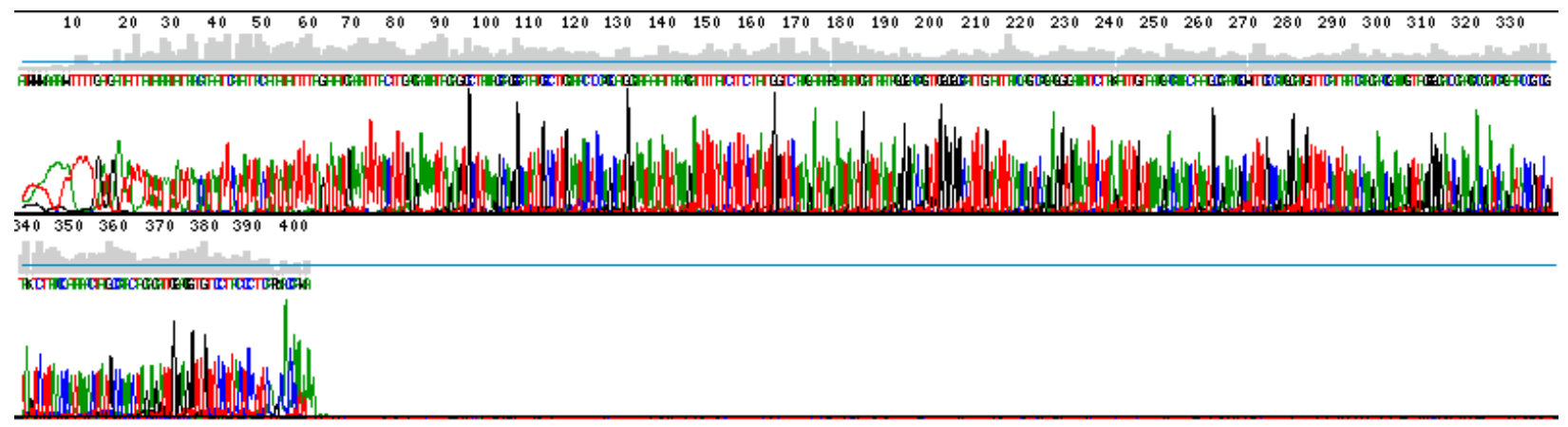

Cattle Sample: 53DaCw

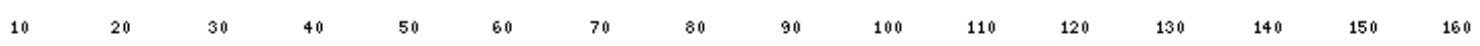

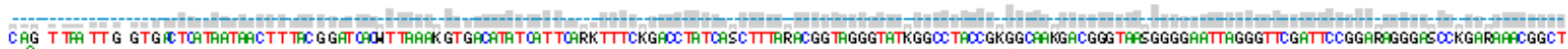

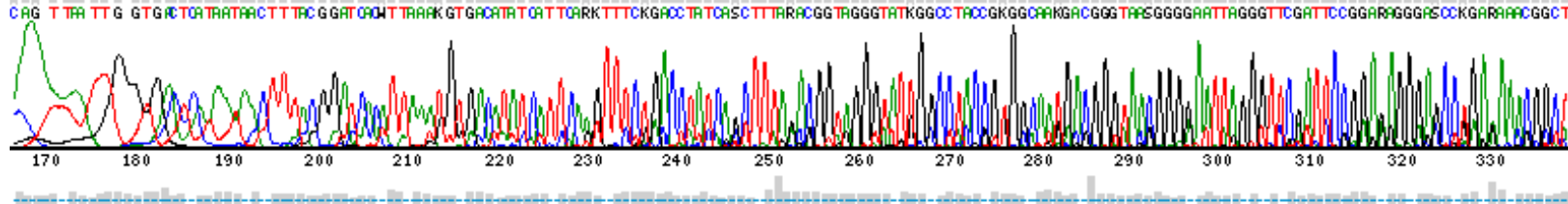

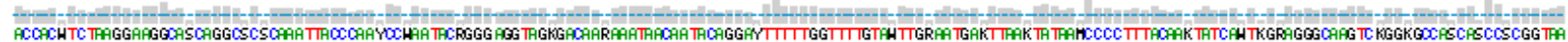

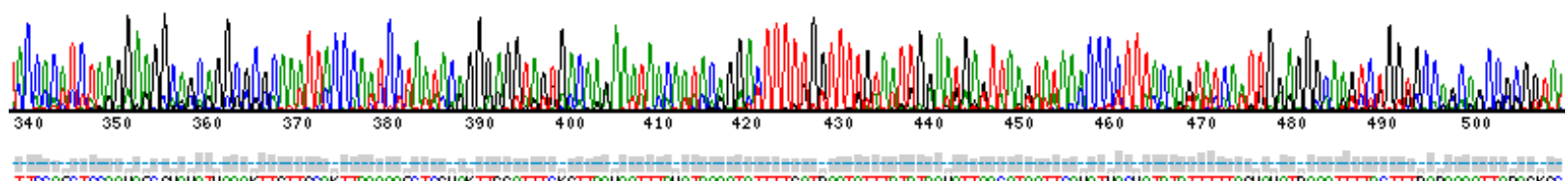

TTC

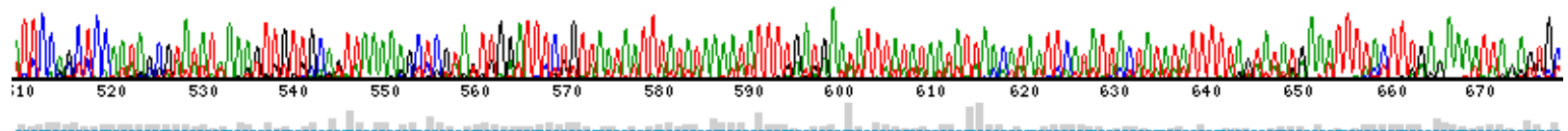

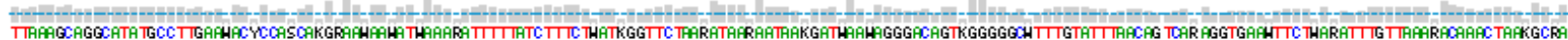

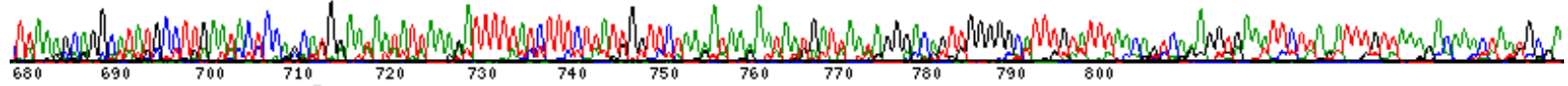

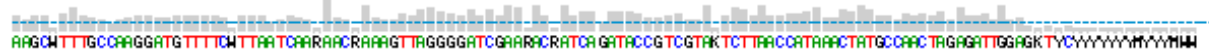

Cattle Sample: $105 \mathrm{Cw}$

Figure E.I.: Chromatogram readings of $18 S$ rRA sequences derived from Cryptosporidium spp. identified in biofilm and cattle samples 


\section{Appendix F: Cost Comparison of EPA Method 1623 and the Biofilm Sampler Technique}

\section{Table F.I.: Approximate Equipment and Service Costs of Cryptosporidium Detection by Method 1623-Filtration/IMS/IFA}

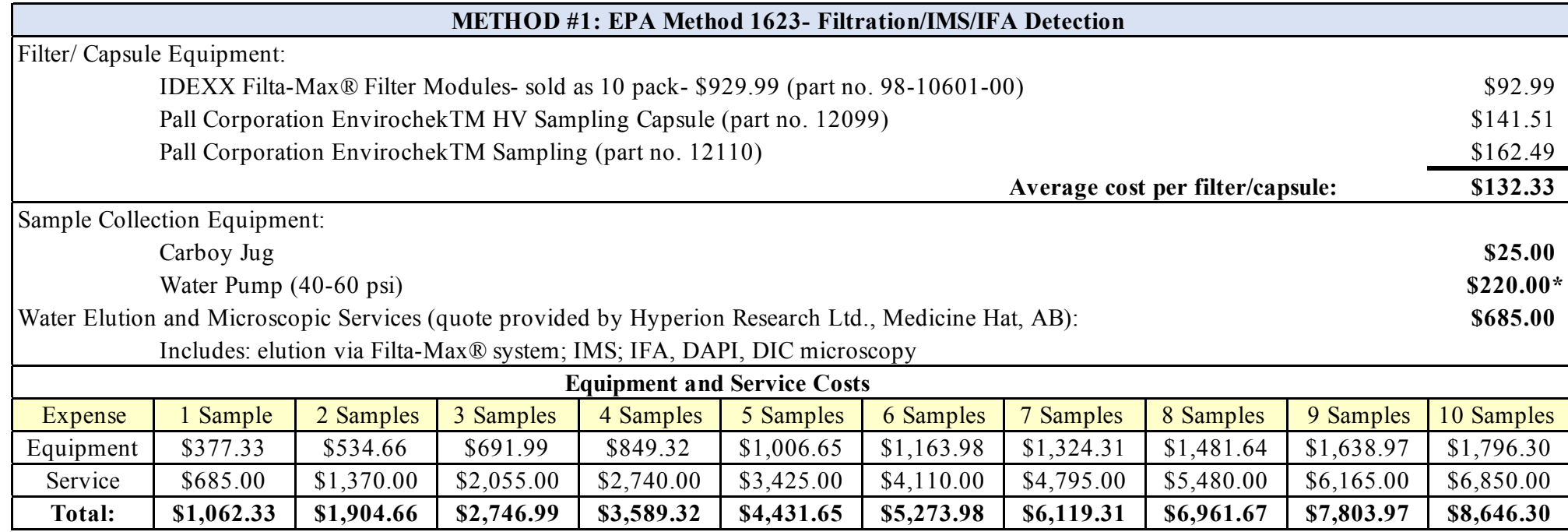

\section{Table F.II.: Approximate Equipment and Service Costs of Cryptosporidium Detection by Biofilm Sampler Technique- IMS/IFA}

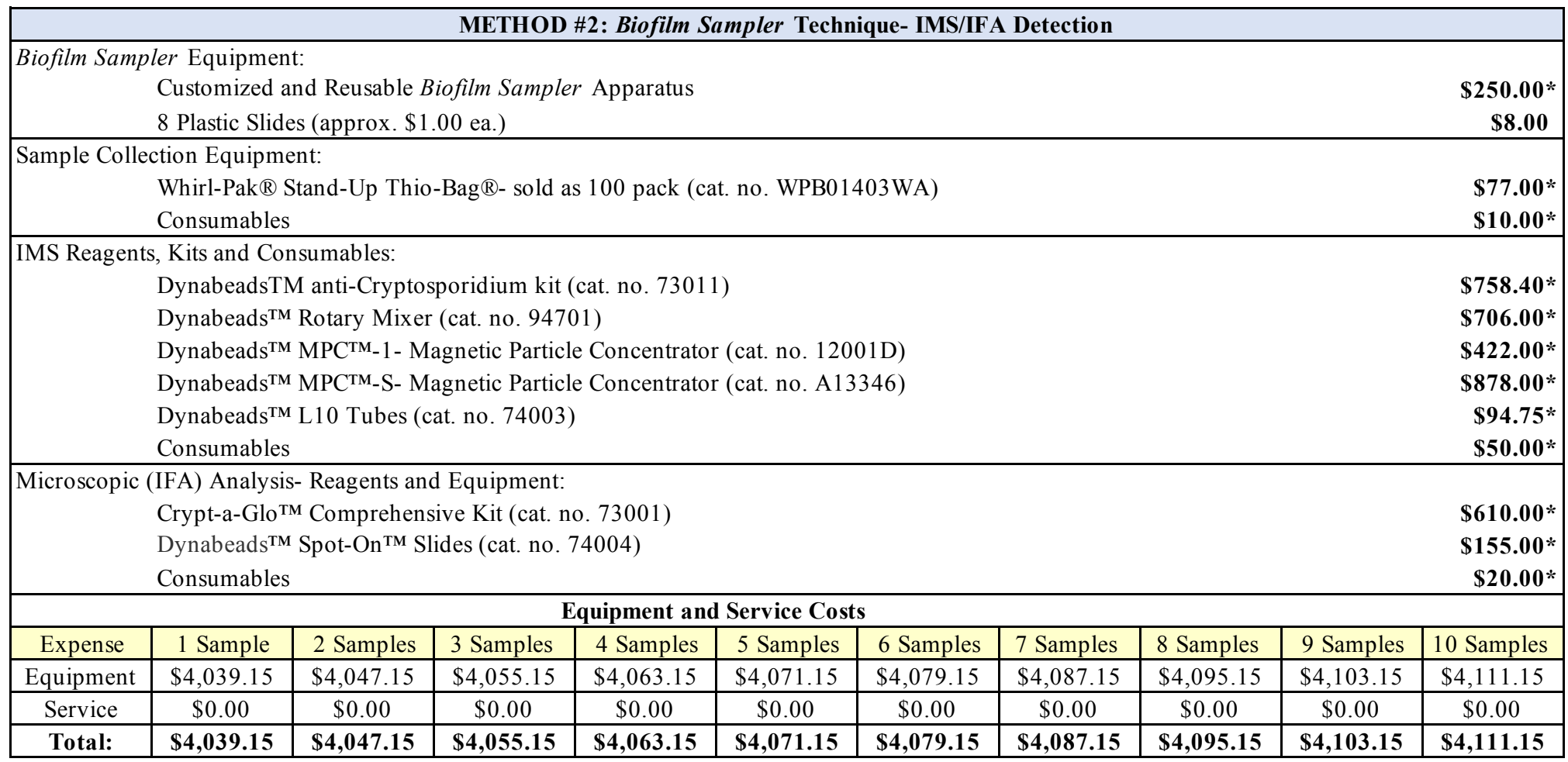




\section{Table F.III.: Approximate Equipment and Service Costs of Cryptosporidium Detection by Biofilm Sampler Technique- IMS/PCR}

\section{METHOD \#3: Biofilm Sampler Technique- IMS/PCR Detection}

Biofilm Sampler Equipment:

Customized and Reusable Biofilm Sampler Apparatus

$\$ 250.00 *$

8 Plastic Slides (approx. $\$ 1.00$ ea.)

Sample Collection Equipment:

Whirl-Pak ${ }^{\circledR}$ Stand-Up Thio-Bag ${ }^{\circledR}$ - sold as 100 pack (cat. no. WPB01403WA)

Consumables

IMS Reagents, Kits and Consumables:

DynabeadsTM anti-Cryptosporidium kit (cat. no. 73011)

Dynabeads $^{\mathrm{TM}}$ Rotary Mixer (cat. no. 94701)

Dynabeads ${ }^{\text {TM }}$ MPCTM 1 - Magnetic Particle Concentrator (cat. no. 12001D)

Dynabeads $^{\mathrm{TM}}$ MPCTM-S- Magnetic Particle Concentrator (cat. no. A13346)

Dynabeads ${ }^{\mathrm{TM}}$ L10 Tubes (cat. no. 74003)

Consumables

PCR Reagents, Kits and Consumables:

QIAamp Fast DNA Stool Mini Kit (cat. no. 51604)

HotStarTaq Master Mix Kit (250 U) (cat. no. 203443)

Primers (quote provided by ACGT Corp.)

Consumables

Electrophoresis Services (quote provided by The Hospital for Sick Children- The Centre for Applied Genomics, Toronto, ON):

8 products $(\$ 2.00$ per sample)

$\$ 16.00$

\begin{tabular}{|c|c|c|c|c|c|c|c|c|c|c|}
\hline \multicolumn{10}{|c|}{ Equipment and Service Costs } \\
\hline Expense & 1 Sample & 2 Samples & 3 Samples & 4 Samples & 5 Samples & 6 Samples & 7 Samples & 8 Samples & 9 Samples & 10 Samples \\
\hline Equipment & $\$ 3,858.88$ & $\$ 3,866.88$ & $\$ 3,874.88$ & $\$ 3,882.88$ & $\$ 3,890.88$ & $\$ 3,898.88$ & $\$ 3,906.88$ & $\$ 3,914.88$ & $\$ 3,922.88$ & $\$ 3,930.88$ \\
\hline Service & $\$ 16.00$ & $\$ 32.00$ & $\$ 48.00$ & $\$ 64.00$ & $\$ 80.00$ & $\$ 96.00$ & $\$ 112.00$ & $\$ 128.00$ & $\$ 144.00$ & $\$ 160.00$ \\
\hline Total: & $\mathbf{\$ 3 , 8 7 4 . 8 8}$ & $\mathbf{\$ 3 , 8 9 8 . 8 8}$ & $\mathbf{\$ 3 , 9 2 2 . 8 8}$ & $\mathbf{\$ 3 , 9 4 6 . 8 8}$ & $\mathbf{\$ 3 , 9 7 0 . 8 8}$ & $\mathbf{\$ 3 , 9 9 4 . 8 8}$ & $\mathbf{\$ 4 , 0 1 8 . 8 8}$ & $\mathbf{\$ 4 , 0 4 2 . 8 8}$ & $\mathbf{\$ 4 , 0 6 6 . 8 8}$ & $\mathbf{\$ 4 , 0 9 0 . 8 8}$ \\
\hline
\end{tabular}

Table F.IV.: Cost Comparison Analysis of MEHODS 1, 2 \& 3

\begin{tabular}{|c|c|c|c|c|c|c|c|c|c|c|}
\hline \multicolumn{11}{|c|}{ Cost- Comparison Analysis } \\
\hline Method no. & 1 Sample & 2 Samples & 3 Samples & 4 Samples & 5 Samples & 6 Samples & 7 Samples & 8 Samples & 9 Samples & 10 Samples \\
\hline METHOD \#1 & $\$ 1,062.33$ & $\$ 1,904.66$ & $\$ 2,746.99$ & $\$ 3,589.32$ & $\$ 4,431.65$ & $\$ 5,273.98$ & $\$ 6,119.31$ & $\$ 6,961.67$ & $\$ 7,803.97$ & $\$ 8,646.30$ \\
\hline METHOD \#2 & $\$ 4,039.15$ & $\$ 4,047.15$ & $\$ 4,055.15$ & $\$ 4,063.15$ & $\$ 4,071.15$ & $\$ 4,079.15$ & $\$ 4,087.15$ & $\$ 4,095.15$ & $\$ 4,103.15$ & $\$ 4,111.15$ \\
\hline METHOD \#3 & $\$ 3,874.88$ & $\$ 3,898.88$ & $\$ 3,922.88$ & $\$ 3,946.88$ & $\$ 3,970.88$ & $\$ 3,994.88$ & $\$ 4,018.88$ & $\$ 4,042.88$ & $\$ 4,066.88$ & $\$ 4,090.88$ \\
\hline \multicolumn{11}{|c|}{ Explanation } \\
\hline \multirow{2}{*}{\multicolumn{11}{|c|}{$\begin{array}{l}\text { METHOD \#1- EPA Method } 1623 \text { (Filtration/IMS/IFA): } \\
\text { Least expensive when analvzing 1-4 samples: Most exnensive when analyzing 5-10 samples }\end{array}$}} \\
\hline & & ve when analy & zing 1-4 san & les; Most ex & nsive when & alyzing $5-10$ & amples & & & \\
\hline \multicolumn{11}{|c|}{ METHOD \#2 \& METHOD \#3- Biofilm Sampler Technique (IMS/IFA \& IMS/PCR): } \\
\hline & Most expen & when ana & g 1-4 sa & ; Least ex & sive when & yzing 5- & & & & \\
\hline
\end{tabular}

* Cost associated with reagents, kits and consumables that can be used more than once

Notes:

i) All quotes are provided in CAD or have been converted from USD to CAD at the conversion rate on 07/14/2017

ii) Analysis does not include the costs of universal microbiology laboratory equipment (e.g., PCR machine, centrifuge, micro-pipettes, votex, glassware etc.)

iii) Consumables refer to the estimated price for disposable laboratory equipment 


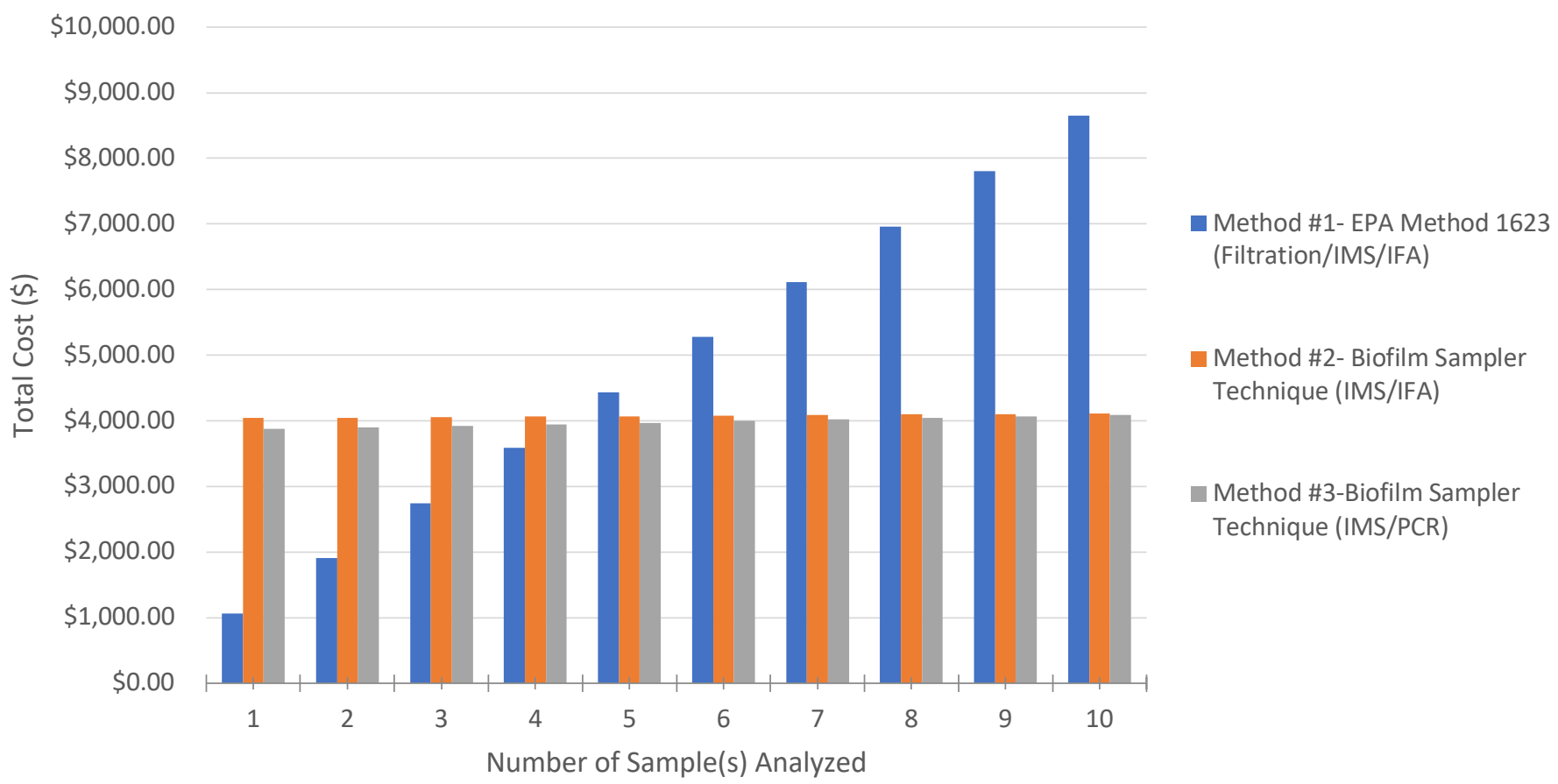

Figure F.I.: Cost-comparison analysis of EPA Method 1623 (filtration/IMS/IFA) and two versions of the biofilm sampler technique (IMS/IFA \& IMS/PCR) 


\section{Appendix G: Photographs of Biofilm Sampling Locations and Cattle Farms}

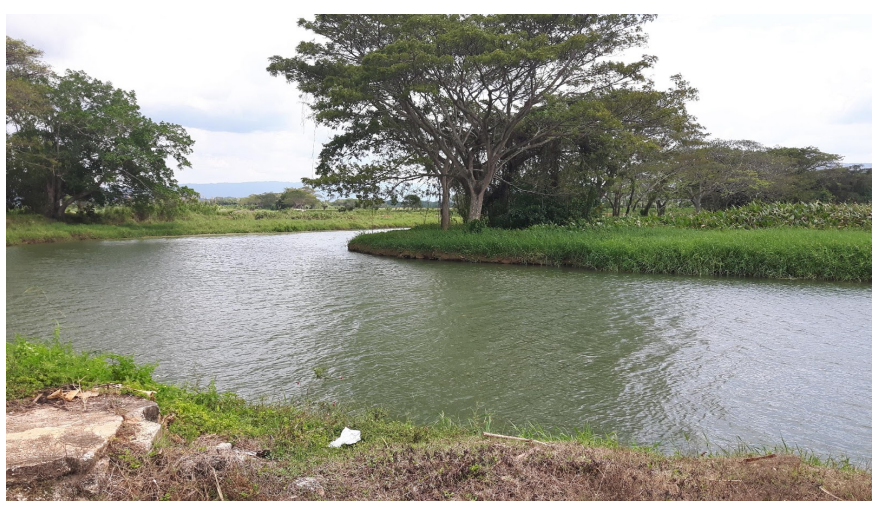

Site A (Middle Quarters)

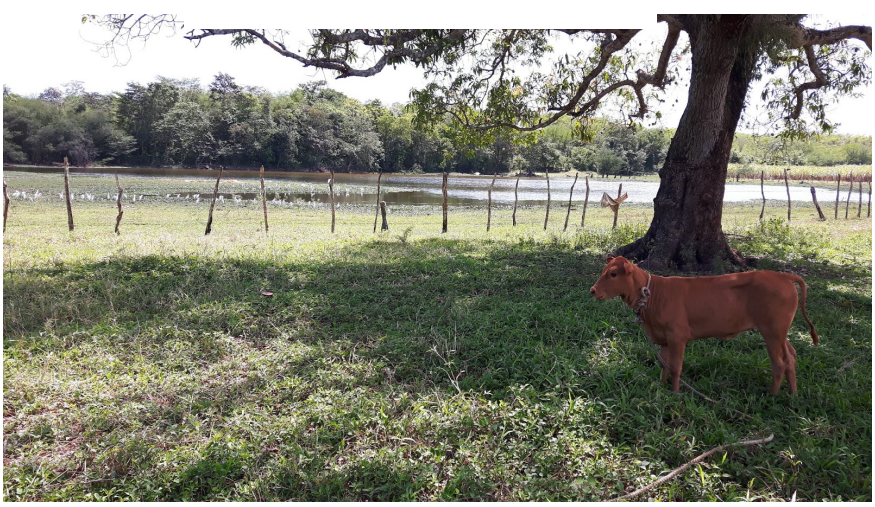

Site C (Black River City)

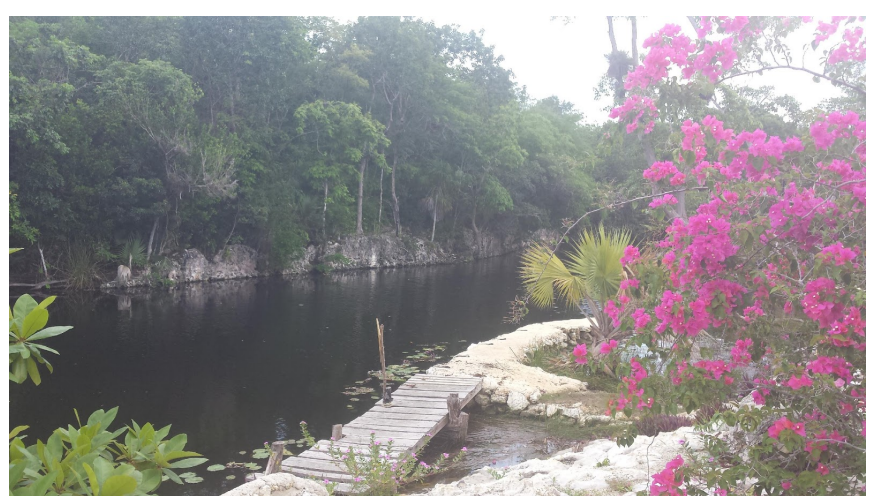

Site B (Cataboo)

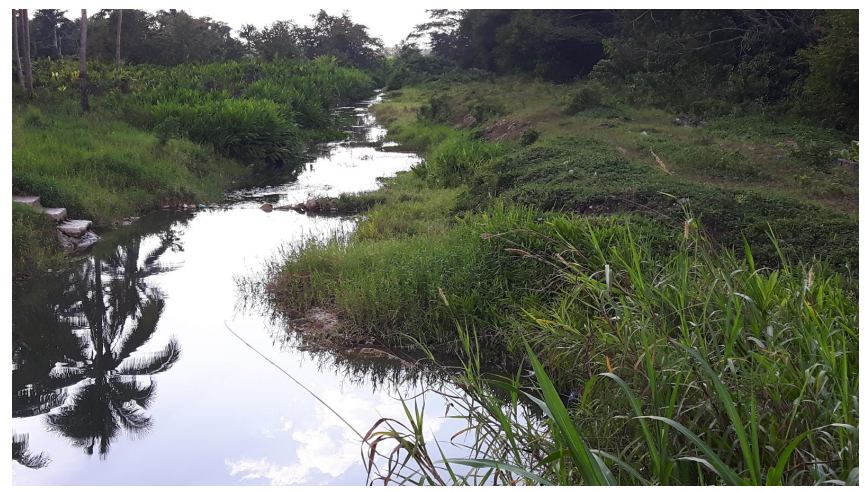

Site D (Lacovia)

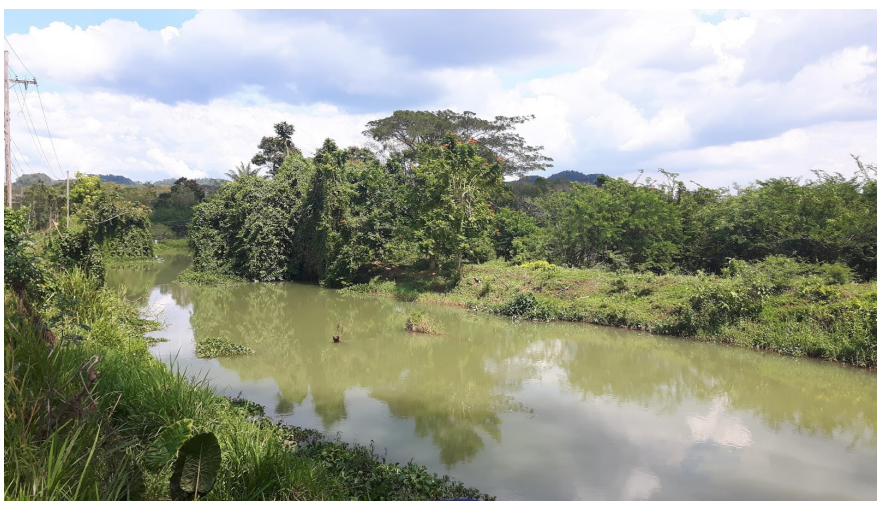

Site E (Bartons)

Figure G.I: Photographs of biofilm sampling sites A-E. Site C highlighting the close proximity of cattle farm to the main river. Site $\mathrm{E}$ illustrating organic waste contamination from large-scale cultivation upstream. 

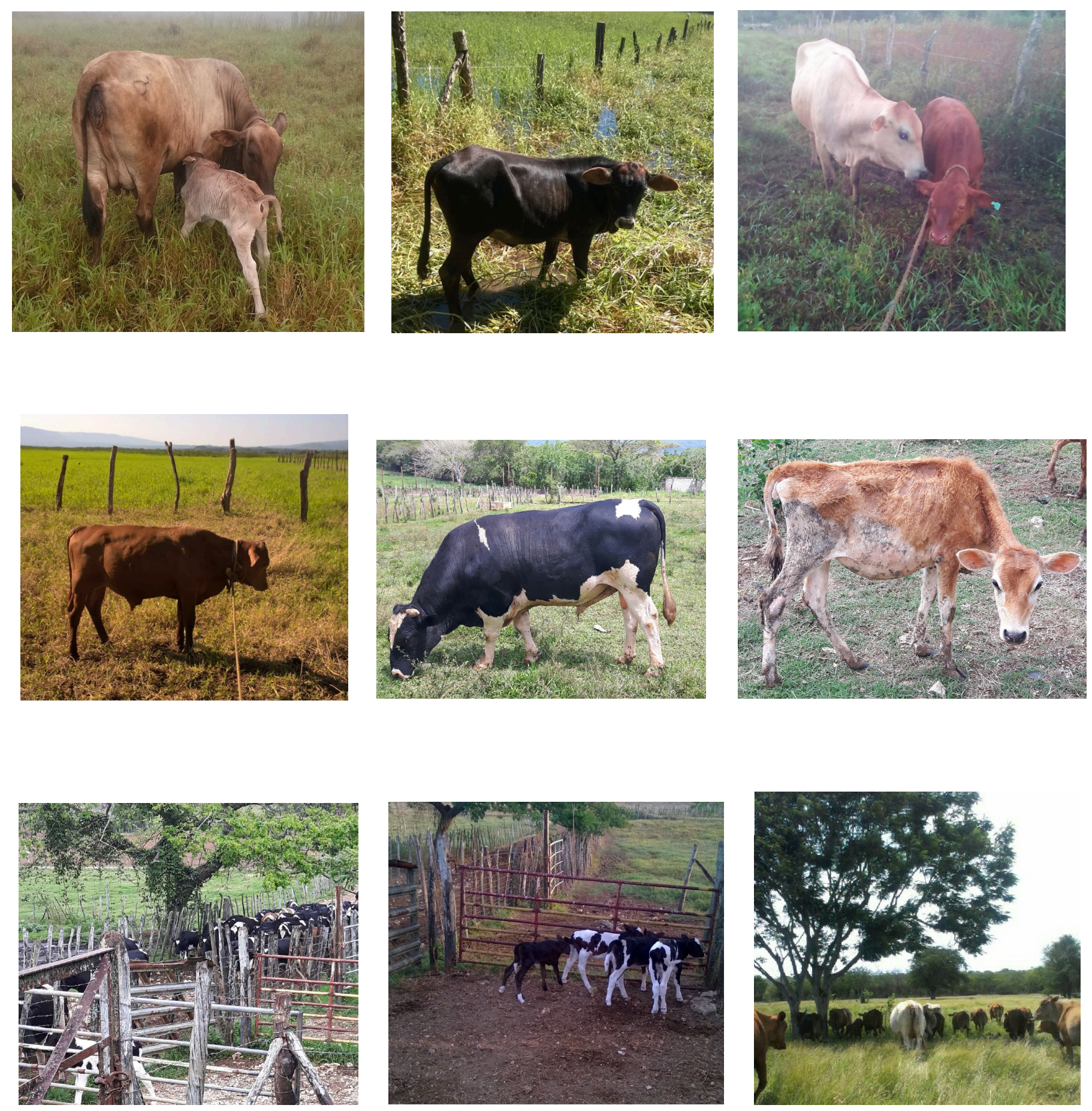

Figure G.II.: Photographs of dairy and beef cattle, and rearing systems. 


\section{Appendix H: 3D Confocal Images of Biofilm Formation}

a
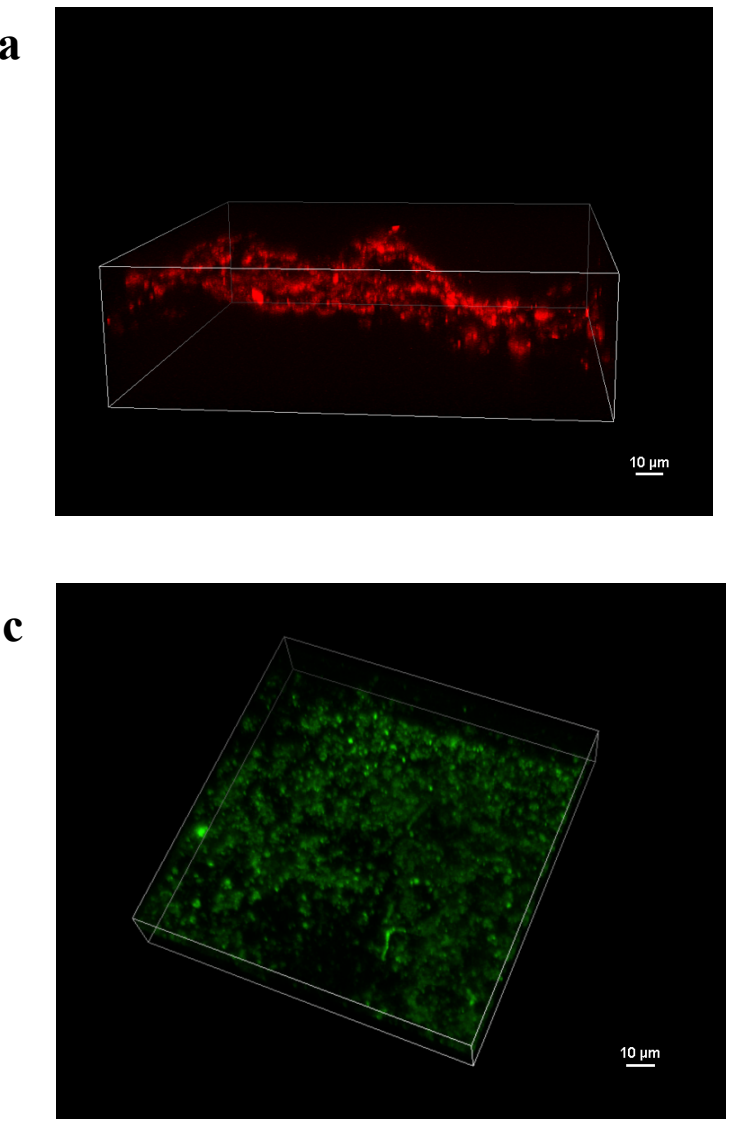

b

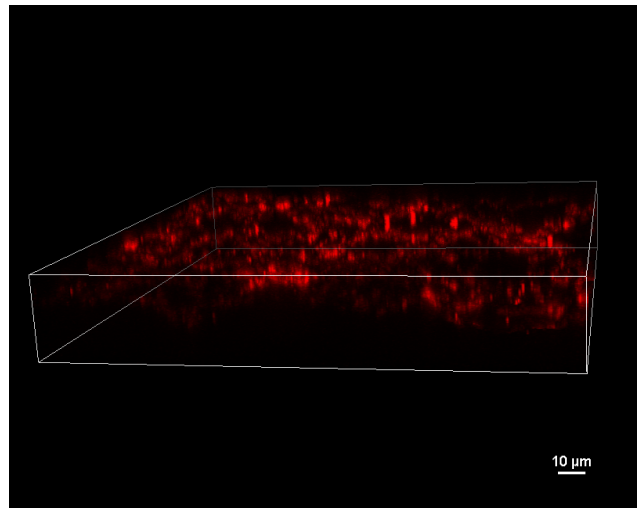

d

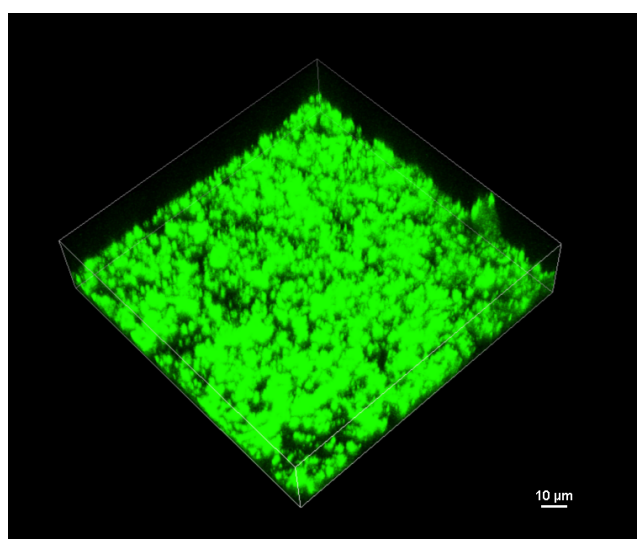

Figure H.I.: Three-dimensional, z-stack imaging of biofilm segments. Captured under confocal microscopy using Streptavidin Texas Red ${ }^{\mathrm{TM}}$ Conjugate and SYTO ${ }^{\mathrm{TM}} 9$ Green Fluorescent Nucleic Acid Stain. Polycarbonate substrate displays an uneven/jagged formation (a, b) with a patchy surface (d). Greater microbial surface coverage was developed on the polycarbonate slide (c) compared to glass microscope slide (d). Magnification, 60X. 


\section{References}

Adamska, M., Leonska-Duniec, A., Sawczuk, M., Maciejewska, A., \& Skotarczak, B. (2012). Recovery of Cryptosporidium from spiked water and stool samples measured by PCR and real time PCR. Veterinarni Medicina, 57(5).

Agnew, D. G., Lima, A. A., Newman, R. D., Wuhib, T., Moore, R. D., Guerrant, R. L., \& Sears, C. L. (1998). Cryptosporidiosis in northeastern Brazilian children: association with increased diarrhea morbidity. The Journal of infectious diseases, 177(3), 754-760.

Akiyoshi, D. E., Dilo, J., Pearson, C., Chapman, S., Tumwine, J., \& Tzipori, S. (2003). Characterization of Cryptosporidium meleagridis of human origin passaged through different host species. Infection and immunity, 71(4), 1828-1832.

Alcantara, C. S., Yang, C. H., Steiner, T. S., Barrett, L. J., Lima, A. A., Chappell, C. L., ... \& Guerrant, R. L. (2003). Interleukin-8, tumor necrosis factor- $\alpha$, and lactoferrin in immunocompetent hosts with experimental and Brazilian children with acquired cryptosporidiosis. The American journal of tropical medicine and hygiene, 68(3), 325328.

Alvarez-Pellitero, P., \& Sitja-Bobadilla, A. (2002). Cryptosporidium molnari n. sp. (Apicomplexa: Cryptosporidiidae) infecting two marine fish species, Sparus aurata L. and Dicentrarchus labrax L. International Journal For Parasitology, 32(8), 1007-1021.

Alves, M., Xiao, L., Sulaiman, I., Lal, A. A., Matos, O., \& Antunes, F. (2003). Subgenotype analysis of Cryptosporidium isolates from humans, cattle, and zoo ruminants in Portugal. Journal of clinical microbiology, 41(6), 2744-2747.

Amer, S., Honma, H., Ikarashi, M., Tada, C., Fukuda, Y., Suyama, Y., \& Nakai, Y. (2010). Cryptosporidium genotypes and subtypes in dairy calves in Egypt. Veterinary parasitology, 169(3-4), 382-386.

Anderson, D., Duszynski, D., \& Marquardt, W. (1968). Three New Coccidia (Protozoa: Telosporea) from Kingsnakes, Lampropeltis spp., in Illinois, with a Redescription of Eimeriu zumenis Phisalix. The Journal of Parasitology, 54(3), 577-581.

Angles, M. L., Chandy, J. P., Cox, P. T., Fisher, I. H., \& Warnecke, M. R. (2007). Implications of biofilm-associated waterborne Cryptosporidium oocysts for the water industry. Trends in parasitology, 23(8), 352-356.

Anguish, L. J., \& Ghiorse, W. C. (1997). Computer-assisted laser scanning and video microscopy for analysis of Cryptosporidium parvum oocysts in soil, sediment, and feces. Applied and environmental microbiology, 63(2), 724-733.

Angus, K. W., Tzipori, S., \& Gray, E. W. (1982). Intestinal lesions in SPF lambs associated with Cryptosporidium from calves with diarrhoea. Veterinary Pathology, 19, 67-688.

Arrowood, M. (2002). In Vitro Cultivation of Cryptosporidium Species. Clinical Microbiology 
Reviews, 15(3), 390- 400.

Ashbolt, N. (2004). Microbial contamination of drinking water and disease outcomes in developing regions. Toxicology, 198, 229-238.

Azeredo, J., Azevedo, N., Briandet, R., Cerca, N., Coenye, T., \& Costa, A. et al. (2016). Critical review on biofilm methods. Critical Reviews In Microbiology, 1-39.

Banda, Z., Nichols, R. A., Grimason, A. M., \& Smith, H. V. (2009). Cryptosporidium infection in non-human hosts in Malawi. Onderstepoort Journal of Veterinary Research, 76(4), 363-375.

Barker, I. K., \& Carbonell, P. L. (1974). Cryptosporidium agni sp. n. from lambs and Cryptosporidium bovis sp. $\mathrm{n}$. from a calf with observations on the oocyst. $Z$ Parasitenkd, 44, 289-298.

Barnes-Pohjonen, R. L. (2012). Use of In-Situ Biofilms to Monitor for Cryptosporidium in Eastern Pennsylavnia Watersheds (Master's dissertation). Retrieved from Theses and Dissertation. 1046.

Behar, S. (1997). Testing the Waters: Chemical and Physical Vital Signs of a River: Definition of Water Quality Parameters. Montpelier. VT: River Watch Network.

Bendinger, B., Rijnaarts, H. H., Altendorf, K., \& Zehnder, A. J. (1993). Physicochemical cell surface and adhesive properties of coryneform bacteria related to the presence and chain length of mycolic acids. Applied and Environmental Microbiology, 59(11), 3973-3977.

Bern, C., Ortega, Y., Checkley, W., Roberts, J.M., Lescano, A.G., Cabrera, L., ...Gilman, R.H. (2002). Epidemiologic differences between cyclosporiasis and cryptosporidiosis in Peruvian children. Emerging Infectious Diseases, 8, 581-585.

Björk, S. (2013). Jamaican Wetlands. Retrieved from http://www.vesan.se/3Bjork/8bj_jamaj.htm

Bonnineau, C., Tlili, A., Faggiano, L., Montuelle, B., \& Guasch, H. (2013). The use of antioxidant enzymes in freshwater biofilms: Temporal variability vs. toxicological responses. Aquatic toxicology, 136, 60-71.

Bouzid, M., Hunter, P. R., Chalmers, R. M., \& Tyler, K. M. (2013). Cryptosporidium pathogenicity and virulence. Clinical microbiology reviews, 26(1), 115-134.

Brentel, A. S., Kantorski, K. Z., Valandro, L. F., Fucio, S. B., Puppin-Rontani, R. M., \& Bottino, M. A. (2011). Confocal laser microscopic analysis of biofilm on newer feldspar ceramic. Operative dentistry, 36(1), 43-51.

Bridge, J. W., Oliver, D. M., Chadwick, D., Godfray, H. C. J., Heathwaite, A. L., Kay, D., ... \& Porter, J. (2010). Engaging with the water sector for public health benefits: waterborne pathogens and diseases in developed countries. Bulletin of the world Health Organization, 88, 873-875. 
Bridgman, S. A., Robertson, R. M. P., Syed, Q., Speed, N., Andrews, N., \& Hunter, P. R. (1995). Outbreak of cryptosporidiosis associated with a disinfected groundwater supply. Epidemiology \& Infection, 115(3), 555-566.

Brown, K. H., Khatun, M., \& Ahmed, G. (1981). Relationship of the xylose absorption status of children in Bangladesh to their absorption of macronutrients from local diets. The American journal of clinical nutrition, 34(8), 1540-1547.

Byrt, T. (1996). How good is that agreement?. Epidemiology, 7(5), 561.

Cacci, S. M., \& Chalmers, R. M. (2016). Human cryptosporidiosis in Europe. Clinical Microbiology and Infection, 22(6), 471-480.

Cacciò, S. M., \& Widmer, G. (Eds.). (2013). Cryptosporidium: Parasite and Disease. Wien, Austria: Springer Science \& Business Media.

Cai, M., Guo, Y., Pan, B., Li, N., Wang, X., Tang, C., ... \& Xiao, L. (2017). Longitudinal monitoring of Cryptosporidium species in pre-weaned dairy calves on five farms in Shanghai, China. Veterinary parasitology, 241, 14-19.

Campbell, A. T., Robertson, L. J., \& Smith, H. V. (1992). Viability of Cryptosporidium parvum oocysts: correlation of in vitro excystation with inclusion or exclusion of fluorogenic vital dyes. Applied and Environmental Microbiology, 58(11), 3488-3493.

Caproco (2003). Bacterial Monitoring General Information. Retrieved from http://www.caproco.com/catalog/pdf/Bacterial-Monitoring/General-Information.pdf.

Caribbean Birding Trail (2015). Black River Great Morass. Caribbean Birding Trail. Retrieved 15 January 2017, from http://www.caribbeanbirdingtrail.org/sites/jamaica/black-rivergreat-morass/

Carreno, R. A., Matrin, D. S., \& Barta, J. R. (1999). Cryptosporidium is more closely related to the gregarines than to coccidia as shown by phylogenetic analysis of apicomplexan parasites inferred using small-subunit ribosomal RNA gene sequences. Parasitology Research, 85(11), 899-904.

CDC (1984). Cryptosporidiosis among children attending daycare centers-Georgia, Pennsylvania, Michigan, California, New Mexico. Morbidity and Mortality Weekly Report, 33, 559-601.

CDC (2016a). Parasites - Cryptosporidium (also known as "Crypto"). Retrieved from https://www.cdc.gov/parasites/crypto/

CDC (2016b). Stool Specimens - Staining Procedures. Retrieved from https://www.cdc.gov/dpdx/diagnosticprocedures/stool/staining.html 
CDC (2016c). Stool Specimens - Detection of Parasite Antigens. Retrieved form https://www.cdc.gov/dpdx/diagnosticprocedures/stool/antigendetection.html

CDC (2017a). Cryptosporidiosis - Chapter 3 - 2018 Yellow Book | Travelers' Health | CDC. Retrieved from https://wwwnc.cdc.gov/travel/yellowbook/2018/infectiousdiseases-related-to-travel/cryptosporidiosis

CDC (2017b). DPDx-Cryptosporidosis. Retrieved from https://www.cdc.gov/dpdx/cryptosporidiosis/index.html

CFSPH (2014). Cryptosporidiosis « CFSPH. Retrieved from http://www.cfsph.iastate.edu/DiseaseInfo/disease.php?name=cryptosporidiosis\&lang=e

Chacín-Bonilla, L., Barrios, F., \& Sanchez, Y. (2008). Environmental risk factors for Cryptosporidium infection in an island from Western Venezuela. Memorias do Instituto Oswaldo Cruz, 103(1), 45-49.

Chalmers, R. M. (2012). Waterborne outbreaks of cryptosporidiosis. Annali dell'Istituto superiore di sanita, 48, 429-446.

Chalmers, R. M., Hadfield, S. J., Jackson, C. J., Elwin, K., Xiao, L., \& Hunter, P. (2008). Geographic linkage and variation in Cryptosporidium hominis. Emerging infectious diseases, 14(3), 496.

Campbell, P. N., \& Current, W. L. (1983). Demonstration of serum antibodies to Cryptosporidium sp. in normal and immunodeficient humans with confirmed infections. Journal of Clinical Microbiology, 18(1), 165-169.

Chappell, C. L., Okhuysen, P. C., Langer-Curry, R. C., Akiyoshi, D. E., Widmer, G., \& Tzipori, S. (2011). Cryptosporidium meleagridis: infectivity in healthy adult volunteers. The American journal of tropical medicine and hygiene, 85(2), 238-242.

Characklis, W. G., McFeters, G. A., \& Marshall, K. C. (1990). Physiological ecology in biofilm systems. Biofilms, 37, 67-72.

Checkley, W., Epstein, L. D., Gilman, R. H., Black, R. E., Cabrera, L., \& Sterling, C. R. (1998). Effects of Cryptosporidium parvum infection in Peruvian children: growth faltering and subsequent catch-up growth. American journal of epidemiology, 148(5), 497-506.

Carpenter, C. R. (2005). Kappa statistic. Canadian Medical Association Journal, 173(1), 15-16.

Clancy, J.L., Gollnitz, W.D. \& Tabib, Z. (1994). Commercial laboratories: how accurate are they?. Journal of American Water Works Association, 86, 89-97.

Clode, P., Koh, W., \& Thompson, R. (2015). Life without a Host Cell: What is Cryptosporidium? Trends In Parasitology, 31(12), 614-624. 
Cole, D. J. (1997). Detection of Cryptosporidium parvum using the Kinyoun acid-fast stain. Proc Annu Conv AAEP, 43, 409-410.

Corso, P., Kramer, M., Blair, K., Addiss, D., Davis, J., \& Haddix, A. (2003). Costs of Illness in the 1993 Waterborne Cryptosporidium Outbreak, Milwaukee, Wisconsin. Emerging Infectious Diseases, 9(4), 426-431.

Comstat 2 (2018). Master Step-by-step guide to using Comstat2. Retrieved from http://www.comstat.dk/helperfiles/Manual-Comstat2--beta.pdf

Considine, R. F., Dixon, D. R., \& Drummond, C. J. (2002). Oocysts of Cryptosporidium parvum and model sand surfaces in aqueous solutions: an atomic force microscope (AFM) study. Water Research, 36(14), 3421-3428.

Coupe, S., Delabre, K., Pouillot, R., Houdart, S., Santillana-Hayat, M., \& Derouin, F. (2006). Detection of Cryptosporidium, Giardia and Enterocytozoon bieneusi in surface water, including recreational areas: a one-year prospective study. FEMS Immunology \& Medical Microbiology, 47(3), 351-359.

Current, W. L. (1985). Cryptosporidiosis. Journal of American Veterinary Medical Association, 187, 1334- 1338.

Current, W. L, Upton, S. J., \& Haynes, T. B. (1986). The life cycle of Cryptosporidium baileyi n. sp. (Apicomplexa, Cryptosporidiidae) infecting chickens. Journal of Protozoology, 33, 289-296.

Dai, X., \& Hozalski, R. (2002). Effect of NOM and biofilm on the removal of Cryptosporidium parvum oocysts in rapid filters. Water Resistance, 36, 3523-3532.

Damiani, C., Balthazard-Accou, K., Clervil, E., Diallo, A., Da Costa, C., Emmanuel, E., ... \& Agnamey, P. (2013). Cryptosporidiosis in Haiti: surprisingly low level of species diversity revealed by molecular characterization of Cryptosporidium oocysts from surface water and groundwater. Parasite, 20.

Daniels, M. E., Smith, W. A., Schmidt, W. P., Clasen, T., \& Jenkins, M. W. (2016). Modeling Cryptosporidium and giardia in ground and surface water sources in rural India: Associations with latrines, livestock, damaged wells, and rainfall patterns. Environmental science \& technology, 50(14), 7498-7507.

Davies, D. G., Parsek, M. R., Pearson, J. P., Iglewski, B. H., Costerton, J. W., \& Greenberg, E. P. (1998). The involvement of cell-to-cell signals in the development of a bacterial biofilm. Science, 280(5361), 295-298.

D'antonio, R. G., Winn, R. E., Taylor, J. P., Gustafson, T. L., Current, W. L., Rhodes, M. M., ... \& Zajac, R. A. (1985). A waterborne outbreak of cryptosporidiosis in normal hosts. Ann Intern Med, 103(6), 886-888.

De Graaf, D., Vanopdenbosch, E., Ortega-Mora, L., Abbassi, H., \& Peeters, J. (1999). A review 
of the importance of cryptosporidiosis in farm animals. International Journal for Parasitology, 29(8), 1269- 1287.

Devane, M. L., Moriarty, E. M., Wood, D., Webster-Brown, J., \& Gilpin, B. J. (2014). The impact of major earthquakes and subsequent sewage discharges on the microbial quality of water and sediments in an urban river. Science of the Total Environment, 485, 666680 .

Delfín, M., Sanjurjo, E., Findlay, C. M., \& Gordeeva, L. M. (1989). Cryptosporidium sp. in children with diarrhea in Cuba. Meditsinskaia parazitologiia i parazitarnye bolezni, (4), 36-39.

Diagnostic Automation, Inc. (2003). Cryptosporidium Antigen Detection ELISA. Retrieved from http://www.rapidtest.com/Cryptosporidium_8301-3.pdf

Díaz-Lee, A., Molina, R., Dougnac, C., Mercado, R., Retamal, P., \& Fredes, F. (2015). Sensibilidad analítica de técnicas de tinción tradicionales y una técnica molecular para la detección de ooquistes de Cryptosporidium spp. aislados de bovinos en muestras de agua: estudio preliminar. Archivos de medicina veterinaria, 47(1), 91-96.

Dietrich, D., Uhl, B., Sailer, V., Holmes, E. E., Jung, M., Meller, S., \& Kristiansen, G. (2013). Improved PCR performance using template DNA from formalin-fixed and paraffinembedded tissues by overcoming PCR inhibition. PLoS One, 8(10), e77771.

DiGiorgio, C. L., Gonzalez, D. A., \& Huitt, C. C. (2002). Cryptosporidium and Giardia recoveries in natural waters by using environmental protection agency method 1623. Applied and Environmental Microbiology, 68(12), 5952-5955.

Di Giovanni, G.D., Hashemi, F.H., Shaw, N.J., Abrams, F.A., LeChevallier, M.W. \& Abbaszadegan, M. (1999). Detection of infectious Cryptosporidium parvum oocysts in surface and filter backwash water samples by immunomagnetic separation and integrated cell culture-PCR. Applied and Environmental Microbiology, 65, 3427-3432

Dixon, B. (2016). Parasitic illnesses associated with the consumption of fresh produce - an emerging issue in developing countries. Current Opinion in Food Science, 8, 104-109.

Dobretsov, S., Teplitski, M., \& Paul, V. (2009). Mini-review: quorum sensing in the marine environment and its relationship to biofouling. Biofouling, 25(5), 413-427.

Dubey, J., \& Pande, B. (1963). Observations on the coccidian oocysts from Indian jungle cat (Felis chaus). Indian Journal of Microbiology, 3, 103-108.

DuPont, H., Chappell, C., Sterling, C., Okhuysen, P., Rose, J., \& Jakubowski, W. (1995). The Infectivity of Cryptosporidium parvum in Healthy Volunteers. New England Journal Of Medicine, 332(13), 855-859.

Ee, R., Madhaiyan, M., Ji, L., Lim, Y. L., Nor, N. M., Tee, K. K., ... \& Yin, W. F. (2016). 
Chania multitudinisentens gen. nov., sp. nov., an N-acyl-homoserine-lactone-producing bacterium in the family Enterobacteriaceae isolated from landfill site soil. International journal of systematic and evolutionary microbiology, 66(6), 2297-2304.

Egorov, A., Frost, F., Muller, T., Naumova, E., Tereschenko, A., \& Ford, T. (2004). Serological evidence of Cryptosporidium infections in a Russian city and evaluation of risk factors for infections. Annals of epidemiology, 14(2), 129-136.

Eisenberg, J. N., Seto, E. Y., Colford Jr, J. M., Olivieri, A., \& Spear, R. C. (1998). An analysis of the Milwaukee cryptosporidiosis outbreak based on a dynamic model of the infection process. Epidemiology, 255-263.

Evans, S., \& Warnow, T. (n.d.). Phylogenetic analyses of alignments with gaps. Retrieved from https://statistics.berkeley.edu/sites/default/files/tech-reports/807.pdf

Fayer, R., \& Nerad, T. (1996). Effects of low temperatures on viability of Cryptosporidium parvum oocysts. Applied Environmental Microbiology, 62, 1431-1433.

Fayer, R., Graczyk, T. K., Lewis, E. J., Trout, J. M., \& Farley, C. A. (1998). Survival of infectious Cryptosporidium parvum oocysts in seawater and eastern oysters (Crassostrea virginica) in the Chesapeake Bay. Applied and Environmental Microbiology, 64(3), 1070-1074.

Fayer, R., \& Ungar, B. L. (1986). Cryptosporidium spp. and cryptosporidiosis. Microbiological Review, 50, 458-483.

Fayer, R., Trout, J., \& Jenkins, M. (1998). Infectivity of Cryptosporidium parvum oocysts stored in water at environmental temperatures. Journal of Parasitology, 84, 1165-1169.

Fayer, R., Trout, J. M., Xiao, L., Morgan, U. M., Lal, A. A., \& Dubey, J.P. (2001). Cryptosporidium canis $\mathrm{n}$. sp. from domestic dogs. Journal of Parasitology, 87, 14151422.

Fayer, R., Santin, M., \& Xiao, L. (2005). Cryptosporidium Bovis n. sp. (Apicomplexa: Cryptosporidiidae) in Cattle (Bos Taurus). Journal Of Parasitology, 91(3), 624-629.

Fayer, R., \& Xiao (2008). Cryptosporidium and Cryptosporidiosis. Boca Raton, FL: CRC Press.

Fayer, R. (2010). Taxonomy and species delimitation in Cryptosporidium. Experimental Parasitology, 124(1), 90-97.

Fayer, R., Santín, M., \& Macarisin, D. (2010). Cryptosporidium ubiquitum n. sp. in animals and humans. Veterinary parasitology, 172(1-2), 23-32.

Ferrari, B. C., Stoner, K., \& Bergquist, P. L. (2006). Applying fluorescence based technology to $t$ he recovery and isolation of Cryptosporidium and Giardia from industrial wastewater streams. Water Research, 40(3), 541-548. 
Fiwi Roots (2016). Jamaica Black River, About the River. Jamaicablackriver.com. Retrieved from http://jamaicablackriver.com/river.html

Flegg, P. J. (1987). Cryptosporidium in travellers from Pakistan. Transactions of the Royal Society of Tropical Medicine and Hygiene, 81(1), 171.

Feng, Y., Ortega, Y., He, G., Das, P., Xu, M., Zhang, X., ... \& Xiao, L. (2007). Wide geographic distribution of Cryptosporidium bovis and the deer-like genotype in bovines. Veterinary parasitology, 144(1-2), 1-9.

Feng, Y., Li, N., Duan, L., \& Xiao, L. (2009). Cryptosporidium genotype and subtype distribution in raw wastewater in Shanghai, China: evidence for possible unique Cryptosporidium hominis transmission. Journal of clinical microbiology, 47(1), 153-157.

Flemming, H. C., \& Wingender, J. (2010). The biofilm matrix. Nature Reviews Microbiology, 8(9), 623-633.

Fletcher, M., \& Loeb, G. I. (1979). Influence of substratum characteristics on the attachment of a marine pseudomonad to solid surfaces. Applied and Environmental Microbiology, 37(1), $67-72$.

Fox, K.R., \& Lytle, D.A. (1996). Milwaukee's Crypto Outbreak: Investigation and Recommendations. American Water Works Association, 88(9), 87-94.

Francy, D. S., Simmons, O. D., Ware, M. W., Granger, E. J., Sobsey, M. D., \& Schaefer, F. W. (2004). Effects of seeding procedures and water quality on recovery of Cryptosporidium oocysts from stream water by using US Environmental Protection Agency Method 1623. Applied and environmental microbiology, 70(7), 4118-4128.

Freire-Santos, F., Gomez-Couso, H., Ortega-Iñarrea, M., Castro-Hermida, J., Oteiza-López, A., Garcia-Martin, O., \& Ares-Mazás, M. (2002). Survival of Cryptosporidium parvum oocysts recovered from experimentally contaminated oysters (Ostrea edulis) and clams (Tapes decussatus). Parasitology research, 88(2), 130-133.

Fuchs, S., Katbeh-Bader, A., \& Alkhateeb, M. (2012). Monitoring of Surface Water Pollution Based on Biological Indicators. http://royalbotanicgarden.org/sites/default/files/files/Monitoring\%20of\%20Surface $\% 20 \mathrm{~W}$ ater\%20Pollution\%20Based\%200n\%20Biological\%20Indicators_RBG\%20Scientific\%20 Day\%202012.pdf

Gatei, W., Barrett, D., Lindo, J., Eldemire-Shearer, D., Cama, V., \& Xiao, L. (2008). Unique Cryptosporidium Population in HIV-Infected Persons, Jamaica. Emerging Infectious Diseases, 14(5), 841-843.

Gatti, S., Cevini, C., Bruno, A., Bernuzzi, A. M., \& Scaglia, M. (1993). Cryptosporidiosis in tourists returning from Egypt and the Island of Mauritius. Clinical infectious diseases, 16(2), 344-345. 
Garro, C. J., Morici, G. E., Utgés, M. E., Tomazic, M. L., \& Schnittger, L. (2016). Prevalence and risk factors for shedding of Cryptosporidium spp. oocysts in dairy calves of Buenos Aires Province, Argentina. Parasite epidemiology and control, 1(2), 36-41.

Ghazy, A. A., Abdel-Shafy, S., \& Shaapan, R. M. (2016). Cryptosporidiosis in Animals and Man: 3. Prevention and Control. Asian Journal of Epidemiology, 9(1-3), 1-9.

Genomics Core Facility. (n.d.). Automated DNA Sequencing Guidelines. Retrieved from https://www.medschool.lsuhsc.edu/genetics/genomicscore/AutomatedDNASequencingG uidelines.pdf

Gertler, M., Dürr, M., Renner, P., Poppert, S., Askar, M., Breidenbach, J., ... \& Chalmers, R. (2015). Outbreak of Cryptosporidium hominis following river flooding in the city of Halle (Saale), Germany, August 2013. BMC infectious diseases, 15(1), 88.

Geurden, T., Berkvens, D., Geldhof, P., Vercruysse, J., \& Claerebout, E. (2006a). A Bayesian approach for the evaluation of six diagnostic assays and the estimation of Cryptosporidium prevalence in dairy calves. Veterinary research, 37(5), 671-682.

Geurden, T., Goma, F. Y., Siwila, J., Phiri, I. G. K., Mwanza, A. M., Gabriël, S., ... \& Vercruysse, J. (2006b). Prevalence and genotyping of Cryptosporidium in three cattle husbandry systems in Zambia. Veterinary parasitology, 138(3-4), 217-222.

Ghazy, A., Shafy, S., \& Shaapan, R., (2015). Cryptosporidiosis in Animals and Man: 1. Taxonomic Classification, Life Cycle, Epidemiology and Zoonotic Importance. Asian Journal Of Epidemiology, 8(3), 48-63.

Ghoshal, U., Jain, V., Dey, A., \& Ranjan, P. (2018). Evaluation of enzyme linked immunosorbent assay for stool antigen detection for the diagnosis of cryptosporidiosis among HIV negative immunocompromised patients in a tertiary care hospital of northern India. Journal of infection and public health, 11(1), 115-119.

Gibson, C., Stadterman, K. L., \& Sykora, J. (1998). Combined sewer overflows: a source of Cryptosporidium and Giardia?. Water Science and Technology, 38(12), 67-72.

Glaberman, S., Moore, J. E., Lowery, C. J., Chalmers, R. M., Sulaiman, I., Elwin, K., ... \& Xiao, L. (2002). Three drinking-water-associated cryptosporidiosis outbreaks, Northern Ireland. Emerging infectious diseases, 8(6), 631.

Gomez-Bautista, M., Ortega-Mora, L. M., Tabares, E., Lopez-Rodas, V., \& Costas, E. (2000). Detection of infectious Cryptosporidium parvum oocysts in mussels (Mytilus galloprovincialis) and cockles (Cerastoderma edule). Applied and environmental microbiology, 66(5), 1866-1870.

Gong, C., Cao, X. F., Deng, L., Li, W., Huang, X. M., Lan, J. C., ... \& Wang, W. B. (2017). Epidemiology of Cryptosporidium infection in cattle in China: a review. Parasite, 24.

Gómez-Couso, H., Méndez-Hermida, F., \& Ares-Mazás, E. (2006). Levels of detection of 
Cryptosporidium oocysts in mussels (Mytilus galloprovincialis) by IFA and PCR methods. Veterinary parasitology, 141(1-2), 60-65.

Graczyk, T. K., Cranfield, M. R., Fayer, R., \& Bixler, H. (1999). House flies (Musca domestica) as transport hosts of Cryptosporidium parvum. The american journal of tropical medicine and hygiene, 61(3), 500-504.

Gradus, S. (2014). Milwaukee, 1993: The Largest Documented Waterborne Disease Outbreak in US History. Water Quality and Health Council. Retrieved from http://www.waterandhealth.org/milwaukee-1993-largest-documented-waterbornedisease-outbreak-history/

Groelz, D., Sobin, L., Branton, P., Compton, C., Wyrich, R., \& Rainen, L. (2013). Non-formalin fixative versus formalin-fixed tissue: a comparison of histology and RNA quality. Experimental and Molecular Pathology, 94(1), 188-194.

Gubbels, M. J., \& Duraisingh, M. T. (2012). Evolution of apicomplexan secretory organelles. International journal for parasitology, 42(12), 1071-1081.

Guerrant, R. (1997). Cryptosporidiosis: an emerging, highly infectious threat. Emerging Infectious Disease Journal, 3, 51-57.

Guerrant, D. I., Moore, S. R., Lima, A. A., Patrick, P. D., Schorling, J. B., \& Guerrant, R. L. (1999). Association of early childhood diarrhea and cryptosporidiosis with impaired physical fitness and cognitive function four-seven years later in a poor urban community in northeast Brazil. The American journal of tropical medicine and hygiene, 61(5), 707713.

Guiguet Leal, D. A., Pereira, M. A., Bueno Franco, R. M., Branco, N., \& Neto, R. (2008). First report of Cryptosporidium spp. oocysts in oysters (Crassostrea rhizophorae) and cockles (Tivela mactroides) in Brazil. Journal of water and health, 6(4), 527-532.

Guy, R.A., Payment, P., Krull, U.J., \& Horgen, P.A. (2003). Real-time PCR for quantification of Giardia and Cryptosporidium in environmental water samples and sewage. Applied and Environmental Microbiology, 69(9), 5178-5185.

Hallier-Soulier, S., \& Guillot, E. (2003). An immunomagnetic separation-reverse transcription polymerase chain reaction (IMS-RT-PCR) test for sensitive and rapid detection of viable waterborne Cryptosporidium parvum. Environ Microbiol, 5, 592-598.

Harp, J., Fayer, R., Pesch, B., \& Jackson, G. (1996). Effect of pasteurization on infectivity of Cryptosporidium parvum oocysts in water and milk. Applied Environmental Microbiology, 62(8), 2866- 2867.

Harper, M., Slaven, J. E., \& Pang, T. W. (2009). Continued participation in an asbestos fibercounting proficiency test with relocatable grid slides. Journal of Environmental Monitoring, 11(2), 434-438. 
Harwood, V. J., Levine, A. D., Scott, T. M., Chivukula, V., Lukasik, J., Farrah, S. R., \& Rose, J. B. (2005). Validity of the indicator organism paradigm for pathogen reduction in reclaimed water and public health protection. Applied and Environmental Microbiology, 71(6), 3163-3170.

Hatalová, E., Valenčáková, A., \& Kalinová, J. (2017). Comparison of PCR primers for the identification of Cryptosporidia species and genotypes in calves. Bulgarian Journal of Veterinary Medicine, 20(1), 154-157.

Hayes, E. B., Matte, T. D., O'Brien, T. R., McKinley, T. W., Logsdon, G. S., Rose, J. B., ... \& Hurwitz, E. S. (1989). Large community outbreak of cryptosporidiosis due to contamination of a filtered public water supply. New England Journal of Medicine, 320(21), 1372-1376.

Hellard, M., Hocking, J., Willis, J., Dore, G., \& Fairley, C. (2003). Risk factors leading to Cryptosporidium infection in men who have sex with men. Sexually transmitted infections, 79(5), 412-414.

Heydorn, A., Nielsen, A. T., Hentzer, M., Sternberg, C., Givskov, M., Ersbøll, B. K., \& Molin, S. (2000). Quantification of biofilm structures by the novel computer program COMSTAT. Microbiology, 146(10), 2395-2407.

Hijjawi, N., Meloni, B., Ryan, U., Olson, M., \& Thompson, R. (2002). Successful in vitro cultivation of Cryptosporidium andersoni: evidence for the existence of novel extracellular stages in the life cycle and implications for the classification of Cryptosporidium. International Journal For Parasitology, 32(14), 1719-1726.

Hijjawi, N., Meloni, B., Ng'anzo, M., Ryan, U., Olson, M., \& Cox, P. et al. (2004). Complete development of Cryptosporidium parvum in host cell-free culture. International Journal For Parasitology, 34(7), 769-777.

Hijjawi, N. (2010). Cryptosporidium: New developments in cell culture. Experimental Parasitology, 124(1), 54-60.

Hijjawi, N., Estcourt, A., Yang, R., Monis, P., \& Ryan, U. (2010). Complete development and multiplication of Cryptosporidium hominis in cell-free culture. Veterinary Parasitology, 169(1-2), 29-36.

Hofstra, N., Bouwman, A., Beusen, A., \& Medema, G. (2013). Exploring global Cryptosporidium emissions to surface water. Science Of The Total Environment, 442, 1019.

Hogan, J. N., Daniels, M. E., Watson, F. G., Conrad, P. A., Oates, S. C., Miller, M. A., ... \& Jessup, D. A. (2012). Longitudinal Poisson regression to evaluate the epidemiology of Cryptosporidium, Giardia, and fecal indicator bacteria in coastal California wetlands. Applied and environmental microbiology, 78(10), 3606-3613.

Holness, C. (2016). Jamaica Parishes - Their Capitals And Landmark Attractions. My-island- 
jamaica.com. Retrieved 15 October 2016, from http://www.my-islandjamaica.com/jamaica_parishes.html

Hong, S., Kim, K., Yoon, S., Park, W. Y., Sim, S., \& Yu, J. R. (2014). Detection of Cryptosporidium parvum in environmental soil and vegetables. Journal of Korean medical science, 29(10), 1367-1371.

Hoover, D., Hoerr, F., Carlton, W., Hinsman, E., \& Ferguson, H. (1981). Enteric cryptosporidiosis in a naso tang, Naso lituratus Block and Schneider. Journal of Fish Disease, 4, 425-428.

House, J. A. (1978). Economic impact of rotavirus and other neonatal disease agents of animals. Journal of the American Veterinary Medical Association, 173, 573-576.

Hsu, B. M., Huang, C., \& Yeh, H. H. (2002). Evaluation of the ICR protozoan method and Method 1623 for detecting Giardia and Cryptosporidium in actual water samples. Water Science and Technology: Water Supply, 2(3), 89-93.

Hu, J., Feng, Y., Ong, S. L., Ng, W. J., Song, L., Tan, X., \& Chu, X. (2004). Improvement of recoveries for the determination of protozoa Cryptosporidium and Giardia in water using method 1623. Journal of microbiological methods, 58(3), 321-325.

Hunt, A. P., \& Parry, J. D. (1998). The effect of substratum roughness and river flow rate on the development of a freshwater biofilm community. Biofouling, 12(4), 287-303.

Ibrahim, R. A. S., Rabab, Z., Gehan, E. E., Mohamed, E. M., Mohamed, E. F., \& Eman, A. (2016). Comparison between modified acid fast staining and antigen detection assay as diagnostic techniques for Cryptosporidium parvum. World J Med Sci, 13(1), 72-8.

ICZN. (2016). Guildines for Case preparation. Iczn.org. Retrieved from http://iczn.org/content/guidelines-case-preparation

IOM (2009). Lessons from Waterborne Disease Outbreaks. In Global issues in water, sanitation, and health (pp. 106-126). Washington, DC: The National Academies Press

Inman, L. R., \& Takeuchi, A. (1979). Spontaneous cryptosporidiosis in an adult female rabbit. Veterinary pathology, 16(1), 89-95.

Iqbal, A., Lim, A. I., Al Mahdy, M., Dixon, B., \& Surin, J. (2012). Epidemiology of cryptosporidiosis in HIV-infected individuals-a global perspective. Journal of AIDS and Clinical Reports, 1(9), 1-16.

Iseki, M. (1979). Cryptosporidium felis sp. n. (Protozoa: Eimeriorina) from the domestic cat. Japanese Journal of Parasitology, 28, 285-307.

Iqbal, J., Hira, P. R., Al-Ali, F., \& Philip, R. (2001). Cryptosporidiosis in Kuwaiti children: seasonality and endemicity. Clinical microbiology and infection, 7(5), 261-266. 
Jakubowski, W., Boutros, S., Faber, W., Fayer, R., \& Ghiorse, W., et al. (1996). Environmental methods for Cryptosporidium. J. Am. Water Works Assoc. 87(9), 107-121.

Jamaica Information Service (2010). NEPA Moves against Potential Polluters of Kingston Harbour. Jamaica Information Service. Retrieved 14 January 2017, from http://jis.gov.jm/nepa-moves-against-potential-polluters-of-kingston-harbour/

Jamaica Observer (2014). St Elizabeth still the bread basket parish. Jamaica Observer, p. 1. Retrieved from http://www.jamaicaobserver.com/news/St-Elizabeth-still-the-breadbasket-parish_16928486

Jellison, K. L., Hemond, H. F., \& Schauer, D. B. (2002). Sources and species of Cryptosporidium oocysts in the Wachusett Reservoir watershed. Applied and environmental microbiology, 68(2), 569-575.

Jenkins, M. B., Bowman, D. D., \& Ghiorse, W. C. (1998). Inactivation of Cryptosporidium parvum oocysts by ammonia. Applied and environmental microbiology, 64(2), 784-788.

Jervish, H., Merrillt, T., \& Sprinzh, H. (1966). Coccidiosis in the guinea pig small intestine due to a Cryptosporidium. American Journal of Veterinary Research, 27, 408-414.

Jiang, J., Alderisio, K.A., Singh, A., \& Xiao, L. (2005). Development of procedures for direct extraction of Cryptosporidium DNA from water concentrates and for relief of PCR inhibitors. Applied and environmental microbiology, 71(3), 1135-1141.

Johnson, D. W., Pieniazek, N. J., \& Rose, J. B. (1993). DNA probe hybridization and PCR detection of Cryptosporidium compared to immunofluorescence assay. Water Science and Technology, 27(3-4), 77-84.

Johnson, D. W., Pieniazek, N. J., Griffin, D. W., Misener, L., \& Rose, J. B. (1995). Development of a PCR protocol for sensitive detection of Cryptosporidium oocysts in water samples. Applied and Environmental Microbiology, 61(11), 3849-3855.

Jokipii, L., Pohjola, S., \& Jokipii, A. M. M. (1983). Cryptosporidium: a frequent finding in patients with gastrointestinal symptoms. The Lancet, 322(8346), 358-361.

JPAT (2016). JPAT > Interactive Virtual Tour > Black River Morass. Jpat-jm.com. Retrieved from http://www.jpat-jm.com/virtour/blackriver/blackriver.html

Kaplan, J. B. (2010). Biofilm Dispersal: Mechanisms, Clinical Implications, and Potential Therapeutic Uses. Journal of Dental Research, 89(3), 205-218.

Karanis, P., Sotiriadou, I., Kartashev, V., Kourenti, C., Tsvetkova, N., \& Stojanova, K. (2006). Occurrence of Giardia and Cryptosporidium in water supplies of Russia and Bulgaria. Environmental Research, 102(3), 260-271.

Khan, S. M., Debnath, C., Pramanik, A. K., Xiao, L., Nozaki, T., \& Ganguly, S. (2010). 
Molecular characterization and assessment of zoonotic transmission of Cryptosporidium from dairy cattle in West Bengal, India. Veterinary parasitology, 171(1-2), 41-47

Kuhn, R. C., Rock, C. M., \& Oshima, K. H. (2002). Effects of pH and magnetic material on immunomagnetic separation of Cryptosporidium oocysts from concentrated water samples. Applied and environmental microbiology, 68(4), 2066-2070.

Keegan, A.R., Fanok, S., Monis, P.T., \& Saint, C.P. (2003). Cell culture-Taqman PCR assay for evaluation of Cryptosporidium parvum disinfection. Applied and Environmental Microbiology, 69(5), 2505-2511.

Kimbell, L. M., Miller, D. L., Chavez, W., \& Altman, N. (1999). Molecular analysis of the 18S rRNA gene of Cryptosporidium serpentis in a wild-caught corn snake (Elaphe guttata guttata) and a five-species restriction fragment length polymorphism-based assay that can additionally discern C. parvum from C. wrairi. Applied and environmental microbiology, 65(12), 5345-5349.

King, B., Keegan, A., Monis, P., \& Saint, C. (2005). Environmental Temperature Controls Cryptosporidium Oocyst Metabolic Rate and Associated Retention of Infectivity. Applied And Environmental Microbiology, 71(7), 3848-3857.

King, B. J., Hoefel, D., Daminato, D. P., Fanok, S., \& Monis, P. T. (2008). Solar UV reduces Cryptosporidium parvum oocyst infectivity in environmental waters. Journal of applied microbiology, 104(5), 1311-1323.

Kirkpatrick, B. D., Noel, F., Rouzier, P. D., Powell, J. L., Pape, J. W., Bois, G., ... \& Powden, C. (2006). Childhood cryptosporidiosis is associated with a persistent systemic inflammatory response. Clinical infectious diseases, 43(5), 604-608.

Kniel, K. E., Sumner, S. S., Lindsay, D. S., Hackney, C. R., Pierson, M. D., Zajac, A. M., ... \& Fayer, R. (2003). Effect of organic acids and hydrogen peroxide on Cryptosporidium parvum viability in fruit juices. Journal of food protection, 66(9), 1650-1657.

Koeck, D. E., Ludwig, W., Wanner, G., Zverlov, V. V., Liebl, W., \& Schwarz, W. H. (2015). Herbinix hemicellulosilytica gen. nov., sp. nov., a thermophilic cellulose-degrading bacterium isolated from a thermophilic biogas reactor. International journal of systematic and evolutionary microbiology, 65(8), 2365-2371.

Koh, W., Clode, P. L., Monis, P., \& Thompson, R. A. (2013). Multiplication of the waterborne pathogen Cryptosporidium parvum in an aquatic biofilm system. Parasites \& vectors, 6(1), 270.

Kolter, R. (2015). A personal perspective on the nature of npj Biofilms and Microbiomes. npj Biofilms and Microbiomes, 1, 15003.

Korpe, P. S., Haque, R., Gilchrist, C., Valencia, C., Niu, F., Lu, M., ... \& Duggal, P. (2016). 
Natural history of cryptosporidiosis in a longitudinal study of slum-dwelling Bangladeshi children: association with severe malnutrition. PLoS neglected tropical diseases, 10(5), e0004564.

Kotloff, K., Blackwelder, W., Nasrin, D., Nataro, J., Farag, T., \& van Eijk, A. et al. (2012). The Global Enter Multicenter Study (GEMS) of Diarrheal Disease in Infants and Young Children in Developing Countries: Epidemiologic and Clinical Methods of the Case/ Control Study. Clinical Infectious Disease, (55)4, S232-S245.

Koudela, B., \& Modry, D. (1998). New species of Cryptosporidium (Apicomplexa: Cryptosporidiidae) from lizards. Folia Parasitologica, 45(2), 93-100.

Krometis, L. A. H., Characklis, G. W., \& Sobsey, M. D. (2009). Identification of particle size classes inhibiting protozoan recovery from surface water samples via US Environmental Protection Agency Method 1623. Applied and environmental microbiology, 75(20), 6619-6621.

Kváč, M., Kestřánová, M., Pinková, M., Květoňová, D., Kalinová, J., Wagnerová, P., ... \& Stenger, B. (2013). Cryptosporidium scrofarum n. sp.(Apicomplexa: Cryptosporidiidae) in domestic pigs (Sus scrofa). Veterinary parasitology, 191(3-4), 218-227.

Kváč, M., Hofmannová, L., Hlásková, L., Květoňová, D., Vítovec, J., McEvoy, J., \& Sak, B. (2014). Cryptosporidium erinacei n. sp. (Apicomplexa: Cryptosporidiidae) in hedgehogs. Veterinary parasitology, 201(1-2), 9-17.

Kváč, M., Kouba, M., \& Vítovec, J. (2006). Age-related and housing-dependence of Cryptosporidium infection of calves from dairy and beef herds in South Bohemia, Czech Republic. Veterinary Parasitology, 137(3-4), 202-209.

Lake, I., Nichols, G., Bentham, G., Harrison, F., Hunter, P., \& Kovats, S. (2007). Cryptosporidiosis decline after regulation, England and Wales, 1989-2005. Emerging Infectious Disease Journal, 13, 623-625.

Lalancette, C., Papineau, I., Payment, P., Dorner, S., Servais, P., Barbeau, B., ... \& Prévost, M. (2014). Changes in Escherichia coli to Cryptosporidium ratios for various fecal pollution sources and drinking water intakes. Water research, 55, 150-161.

Lang, D., \& MAL-ED Network Investigators. (2015). Opportunities to assess factors contributing to the development of the intestinal microbiota in infants living in developing countries. Microbial ecology in health and disease, 26(1), 28316.

Langmark, J., Storey, M., Ashbolt, N., \& Stenstrom, T. (2005). Accumulation and Fate of Microorganisms and Microspheres in Biofilms Formed in a Pilot-Scale Water Distribution System. Applied And Environmental Microbiology, 71(2), 706-712.

Le Chevallier, M.W., Norton, W.D., Siegel, J.E., Abbaszadegan, M. (1995). Evaluation of the immunofluorescence procedure for detection of Giardia cysts and Cryptosporidium oocysts in water. Appl Environ Microbiol, 61(2), 690-697. 
Le Chevallier, M., Di Giovanni, G., Clancy, J., Bukhari, Z., Bukhari, S. et al. (2003). Comparison of Method 1623 and Cell Culture-PCR for Detection of Cryptosporidium spp. in Source Waters. Applied And Environmental Microbiology, 69(2), 971-979.

Leitch, G., \& He, Q. (2012). Cryptosporidiosis-an overview. Journal Of Biomedical Research, 25(1), 1-16.

Leland, D., McAnulty, J., Keene, W., \& Stevens, G. (1993). A cryptosporidiosis outbreak in a filtered-water supply. American Water Works Association, 85 (6), 34 - 42.

Levine, N. (1980). Some corrections of coccidian (Apicomplexa: Protozoa) nomenclature. Journal of Parasitology, 66, 830-834.

Lewis, F. \& Dunkley, D. (2006). Steps Taken to Improve Environmental Practices at the Appleton Sugar Factory. Retrieved from http://www.jamaicasugar.org/SIRISection/JAST/JASTPapers/papers/Y2006/E

Li, N., Xiao, L., Wang, L., Zhao, S., \& Zhao, X. et al (2012). Molecular surveillance of Cryptosporidium spp., Giardia duodenalis, and Enterocytozoon bieneusi by genotyping and subtyping parasites in wastewater. PLoS Negl Trop Dis, 6(9), e1809.

Lindo, J., Levy, V., Baum, M., \& Palmer, C. (1998). Epidemiology of giardiasis and cryptosporidiosis in Jamaica. The American Journal of Tropical Medicine and Hygiene, 59, 717-721.

Lindsay, D. S., Blagburn, B. L., \& Sundermann, C. A. (1989). Morphometric comparison of the oocysts of Cryptosporidium meleagridis and Cryptosporidium baileyi from birds. In Proc. Helminthol. Soc. Wash, 56 (1), 91-92.

Lindsay, D., Upton, S., Owens, D., Morgan, U., Mead, J., \& Blagburn, B. (2000). Cryptosporidium andersoni n. sp. (Apicomplexa: Cryptosporiidae) from Cattle, Bos taurus. The Journal of Eukaryotic Microbiology, 47(1), 91-95.

Lowery, C.J., Nugent, P., Moore, J. E., Millar, B. C., Xiru, X., \& Dooley, J. S. G. (2001). PCRIMS detection and molecular typing of Cryptosporidium parvum recovered from a recreational river source and an associated mussel (Mytilus edulis) bed in Northern Ireland. Epidemiology and infection, 127(03), 545-553.

Luo, X., Jedlicka, S., \& Jellison, K. (2016). Pseudo-second-order calcium-mediated Cryptosporidium parvum oocyst attachment to environmental biofilms. Applied And Environmental Microbiology, AEM.02339-16.

Ma, P., Kaufman, D. L., Helmick, C. G., d'Souza, A. J., \& Navin, T. R. (1985). Cryptosporidiosis in tourists returning from the Caribbean. The New England journal of medicine, 312(10), 647-648.

Ma, P., \& Soave, R. (1983). Three-step stool examination for cryptosporidiosis in 10 
homosexual men with protracted watery diarrhea. Journal of Infectious Diseases, 147(5), 824-828.

Mac Kenzie, W.R., Hoxie, N.J., Proctor, M.E., et al. (1994). A massive out-break in Milwaukee of Cryptosporidium infection transmitted through the public water supply. $N$ Engl J Med, $331,161-167$.

Marahatta, S. B. (2015). Control of the outbreak of disease aftermath earthquake: an overview. Nepal journal of epidemiology, 5(2), 468.

Marsalek, J., Watt, W. E., Zeman, E., \& Sieker, H. (Eds.). (2012). Advances in urban stormwater and agricultural runoff source controls (Vol. 6). Springer Science \& Business Media.

Martin, N. C., Pirie, A. A., Ford, L. V., Callaghan, C. L., McTurk, K., Lucy, D., \& Scrimger, D. G. (2006). The use of phosphate buffered saline for the recovery of cells and spermatozoa from swabs. Science \& justice: journal of the Forensic Science Society, 46(3), 179-184.

Mawdsley, J., Brooks, A., \& Merry, R. (1996). Movement of the protozoan pathogen Cryptosporidium parvum through three contrasting soil types. Biology and Fertility of Soils, 21, 30- 36 .

Mayer, C. L., \& Palmer, C. J. (1996). Evaluation of PCR, nested PCR, and fluorescent antibodies for detection of Giardia and Cryptosporidium species in wastewater. Applied and Environmental Microbiology, 62(6), 2081-2085.

McAllister, T. A., Olson, M. E., Fletch, A., Wetzstein, M., \& Entz, T. (2005). Prevalence of Giardia and Cryptosporidium in beef cows in southern Ontario and in beef calves in southern British Columbia. The Canadian veterinary journal, 46(1), 47.

McCuin, R. M., \& Clancy, J. L. (2003). Modifications to United States Environmental Protection Agency methods 1622 and 1623 for detection of Cryptosporidium oocysts and Giardia cysts in water. Applied and environmental microbiology, 69(1), 267-274.

McLeod, C. M. (2011). Detection and Genotyping of Cryptosporidium Oocysts in Eastern Pennsylvania Water Supplies. (Master's Dissertation). Retrieved from Theses and Dissertations. 1169.

McDonald, V., Stables, R., Warhurst, D. C., Barer, M. R., Blewett, D. A., Chapman, H. D., ... \& McAdam, K. P. (1990). In vitro cultivation of Cryptosporidium parvum and screening for anticryptosporidial drugs. Antimicrobial agents and chemotherapy, 34(8), 1498-1500.

MEGA Software (2011). Bootstrap Test of Phylogeny. Retrieved from https://www.megasoftware.net/mega4/WebHelp/helpfile.htm\#part_iv__evolutionary_an alysis/constructing_phylogenetic_trees/statistical_tests_of_a_tree_obtained/bootstrap_test s/hc_bootstrap_test_phylogeny.htm

Meisel, J. L., Perera, D. R., Meligro, C., \& Rubin, C. E. (1976). Overwhelming watery diarrhea 
associated with a Cryptosporidium in an immunosuppressed patient. Gastroenterology, 70, 1156-1160.

Mendonça, C., Almeida, A., Castro, A., de Lurdes Delgado, M., Soares, S., \& da Costa, J. M. C. (2007). Molecular characterization of Cryptosporidium and Giardia isolates from cattle from Portugal. Veterinary parasitology, 147(1-2), 47-50

Merod, R. T., Warren, J. E., McCaslin, H., \& Wuertz, S. (2007). Toward automated analysis of biofilm architecture: bias caused by extraneous confocal laser scanning microscopy images. Applied and environmental microbiology, 73(15), 4922-4930.

Meuten, D. J., Van Kruiningen, N.J., \& Lein, D. H. (1974). Cryptosporidiosis in a calf. Journal of the American Veterinary Medical Association, 165, 914-917.

Miao, Y., Awad-El-Kariem, F., Franzen, C., Ellis, D., Muller, A., Counihan, H., et al. (2000). Eradication of cryptosporidia and microsporidia following successful antiretroviral therapy. Journal of Acquired Immune Deficiency Syndrome, 25, 124-129.

Miller, W. A., Atwill, E. R., Gardner, I. A., Miller, M. A., Fritz, H. M., Hedrick, R. P., ... \& Conrad, P. A. (2005). Clams (Corbicula fluminea) as bioindicators of fecal contamination with Cryptosporidium and Giardia spp. in freshwater ecosystems in California. International journal for parasitology, 35(6), 673-684.

Miller, W. A., Gardner, I. A., Atwill, E. R., Leutenegger, C. M., Miller, M. A., Hedrick, R. P., ... \& Conrad, P. A. (2006). Evaluation of methods for improved detection of Cryptosporidium spp. in mussels (Mytilus californianus). Journal of microbiological methods, 65(3), 367-379.

Miller, N., Waite, L., \& Harlan, A. (2001). Water Resources Assessment of Jamaica. Retrieved from http://www.sam.usace.army.mil/Portals/46/docs/military/engineering/docs/WRA/Jamaica /Jamaica\%20WRA\%20-\%20English.pdf

Miyazaki, N. (2005). Mankind and the oceans. Tokyo: United Nations University Press.

Modini, L., Pizarro, A., Pizarro, M., \& Zerbatto, M. (2016). Longevity of Cryptosporidium oocysts in fresh and sea water at environmental temperatures. Journal Bacteriol Parasitology, 7(3), 102-107

Moore, D. A., Atwill, E. R., Kirk, J. H., Brahmbhatt, D., Alonso, L. H., Hou, L., ... \& Miller, T. D. (2003). Prophylactic use of decoquinate for infections with Cryptosporidium parvum in experimentally challenged neonatal calves. Journal of the American Veterinary Medical Association, 223(6), 839-845.

Mordab, P., Hong Yuan, T., \& Hu, X. (2013). Jamaican Wetlands. Vesan.se. Retrieved 9 January 2017, from http://www.vesan.se/3Bjork/8bj_jamaj.htm

Morgan, U.M., Constantine, C.C., Forbes, D.A., \& Thompson, R.C. (1997). Differentiation 
between human and animal isolates of Cryptosporidium parvum using rDNA sequencing and direct PCR analysis. The Journal of parasitology, 83(5), 825-830.

Morgan, U. M., \& Thompson, R. C. A. (1998). PCR detection of Cryptosporidium: the way forward?. Parasitology Today, 14(6), 241-245.

Morgan, U. M., Monis, P. T., Fayer, R., Deplazes, P., \& Thompson, R. A. (1999). Phylogenetic relationships among isolates of Cryptosporidium: evidence for several new species. The Journal of parasitology, 1126-1133.

Morgan-Ryan, U., Fall, A., Ward, L., Hijjawi, N., Sulaiman, I., \& Fayer, R. et al. (2002). Cryptosporidium hominis n. sp. (Apicomplexa: Cryptosporidiidae) from Homo sapiens. Journal of Eukaryotic Microbiology, 49, 433-440.

Morgan, U., Weber, R., Xiao, L., Sulaiman, I., Thompson, R. A., Ndiritu, W., ... \& Deplazes, P. (2000). Molecular characterization of Cryptosporidium isolates obtained from human immunodeficiency virus-infected individuals living in Switzerland, Kenya, and the United States. Journal of Clinical Microbiology, 38(3), 1180-1183.

Morin, M., Lariviere, S., \& Lather, R. (1976). Pathological and microbiological observations made on spontaneous cases of acute neonatal calf diarrhea. Canadian Journal of Comparative Medicine, 40, 228-240.

Mosnier, E., Martin, N., Razakandrainibe, R., Dalle, F., Roux, G., Buteux, A., ... \& Epelboin, L. (2018). Cryptosporidiosis Outbreak in Immunocompetent Children from a Remote Area of French Guiana. The American journal of tropical medicine and hygiene, 98(6), 17271732.

Moura, L. (2010). Algorithms in Bioinformatics: Lecture 15-16: Phylogeny Reconstruction [PowerPoint slides]. Retrieved from http://www.site.uottawa.ca/ lucia/courses/512611/lecturenotes/16-17PhylogenyReconstruction.pdf

Murga, R., Stewart, P. S., \& Daly, D. (1994). Quantitative analysis of biofilm thickness variability. Biotechnology and bioengineering, 45(6), 503-510.

Nagy, B., Bozso, M., Pal, V., Nagy, G., \& Sahibi, M.A. (1984). Studies on cryptosporidial infection of goat kids. In: YvoreÂ P, Perrini G, editors. Les maladies de la cheÁvre. INRA Publications, 443-451.

Nair, P., Mohamed, J. A., DuPont, H. L., Figueroa, J. F., Carlin, L. G., Jiang, Z. D., ... \& Okhuysen, P. C. (2008). Epidemiology of cryptosporidiosis in North American travelers to Mexico. The American journal of tropical medicine and hygiene, 79(2), 210-214.

Nannini, E., \& Okhuysen, P. (2002). HIV1 and the gut in the era of highly active antiretroviral therapy. Current Gastroenterology, 4, 392-398.

National Geographic Society (2017). Ocean Acidification. Retrieved from 
https://www.nationalgeographic.com/environment/oceans/critical-issues-oceanacidification/

Navin, T. R., \& Juranek, D. D. (1984). Cryptosporidiosis: clinical, epidemiologic, and parasitologic review. Reviews on Infectious Diseases, 6, 313-327.

Neill, M. A., Rice, S. K., Ahmad, N. V., \& Flanigan, T. P. (1996). Cryptosporidiosis: an unrecognized cause of diarrhea in elderly hospitalized patients. Clinical infectious diseases, 22(1), 168-170.

NEPA (2003). Watershed Policy For Jamaica. Retrieved from http://www.nepa.gov.jm/projects/R2RW/R2RW\%20CD\%20-\%2002/031/031.pdf

NEPA (2010). State of the Environment Report 2010. Retrieved from http://www.nepa.gov.jm/publications/SOE/2010/state-of-The-environment-report-2010Jamaica.pdf

NEPA (2014). Watershed Management Unit [Photograph]. Retrieved from http://nepa.gov.jm/

NEPA (2015). NEPA Serves Wray And Nephew's Appleton Estate Enforcement Notice. Nepa.gov.jm. Retrieved from http://www.nepa.gov.jm/newscenter/Press_releases/current/PR20150330nepa_serves_wray_and_nephew_enforcement\%20notice.asp

NEPA (2017). National Environment \& Planning Agency. Nepa.gov.jm. Retrieved 11 January 2017, from http://www.nepa.gov.jm/

Ngouanesavanh, T., Guyot, K., Certad, G., FICHOUX, Y. L., Chartier, C., VERDIER, R. I., ... \& BAÑULS, A. L. (2006). Cryptosporidium population genetics: evidence of clonality in isolates from France and Haiti. Journal of Eukaryotic Microbiology, 53, S33-S36.

Nichols, R. A. B., Campbell, B. M., \& Smith, H. V. (2003). Identification of Cryptosporidium spp. oocysts in United Kingdom noncarbonated natural mineral waters and drinking waters by using a modified nested PCR-restriction fragment length polymorphism assay. Applied and environmental microbiology, 69(7), 4183-4189.

Nikaeen, M., Mesdaghinia, A.R., Tehrani, M.J., Rezaeian, M., \& Makimura, K. (2005). A Nested-PCR assay for detection of Cryptosporidium parvum oocysts in water samples. Iranian Journal of Public Health, 34(1), 13-18

Nime, F. A., Burek, J. D., Page, D. L., Holscher, M. A., \& Yardley, J. H. (1976). Acute enterocolitis in a human being infected with the protozoan Cryptosporidium. Gastroenterology, 70, 592-598.

Núñez, F. A., González, O. M., González, I., Escobedo, A. A., \& Cordoví, R. A. (2003a). Intestinal coccidia in Cuban pediatric patients with diarrhea. Memórias do Instituto Oswaldo Cruz, 98(4), 539-542. 
Núñez, F. A., González, O. M., Bravo, J. R., Escobedo, A. A., \& Gonzaléz, I. (2003b). Intestinal parasitosis in children admitted to the Pediatric Teaching Hospital of Cerro, Havana City, Cuba. Revista cubana de medicina tropical, 55(1), 19-26.

Ochoa, T.J., Salazar-Lindo, E., \& Cleary, T.G. (2004). Management of children with infectionassociated persistent diarrhea. Seminars in Pediatric Infectious Diseases, 15, 229-236.

O’Brien, E., McInnes, L., \& Ryan, U. (2008). Cryptosporidium GP60 genotypes from humans and domesticated animals in Australia, North America and Europe. Experimental parasitology, 118(1), 118-121.

O’Donoghue, P. (1995). Cryptosporidium and cryptosporidiosis in man and animals. International Journal For Parasitology, 25(2), 139-195.

O'Handley, R.M., Cockwill, C., McAllister, T.A., Jelinski, M., Morck, D.W., \& Olson, M. E. (1999). Duration of naturally acquired giardiosis and cryptosporidiosis in dairy calves and their association with diarrhea. Journal of the American Veterinary Medical Association, 214(3), 391-396.

Okamoto, N., \& Keeling, P. J. (2014). The 3D structure of the apical complex and association with the flagellar apparatus revealed by serial TEM tomography in Psammosa pacifica, a distant relative of the Apicomplexa. PloS one, 9(1), e84653.

Olson, M. E., Ralston, B. J., R. O’Handley, Guselle, N. J. \& Appelbee, A. J. (2003). What is the Clinical and Zoonotic Significance of Cryptosporidiosis in Domestic Animals and Wildlife. In R. A. Thompson, A. Armson \& U. M. Ryan (Eds.), Cryptosporidium: From Molecules to Disease (pp. 53-54). Amsterdam, Netherlands: Elsevier.

Osaki, S.C., Soccol, V.T., Costa, A.O., Oliveira-Silva, M. B., Pereira, J. T., \& Procopio, A. E. (2013). Polymerase chain reaction and nested-PCR approaches for detecting Cryptosporidium in water catchments of water treatment plants in Curitiba, State of Paraná, Brazil. Revista da Sociedade Brasileira de Medicina Tropical, 46(3), 270-276.

Pall Corporation (2017). EnvirochekTM and Envirochek HV Sampling Capsules and Accessories. Retrieved from https://www.pall.com/pdfs/Laboratory/20088_PN33142_.pdf

Pape, J. W., Levine, E., Beaulieu, M. E., Marshall, F., Verdier, R., \& Johnson Jr, W. D. (1987). Cryptosporidiosis in Haitian children. The American journal of tropical medicine and hygiene, 36(2), 333-337.

Pape, J., \& Johnson Jr, W. D. (1993). AIDS in Haiti: 1982-1992. Clinical Infectious Diseases, 17(Supplement_2), S341-S345

Panciera, R. J., Thomassen, R.W., \& Garner F. M. (1971). Cryptosporidial infection in a calf. Veterinary Pathology, 8, 479-484.

Pang, T. W. (1996). Canada Patent Application No. 2124613. Gatineau, QC. Canadian 
Intellectual Property Office.

Pang, T. W., \& Harper, M. (2008). The quality of fiber counts using improved slides with relocatable fields. Journal of Environmental Monitoring, 10(1), 89-95.

Park, J. H., Guk, S. M., Han, E. T., Shin, E. H., Kim, J. L., \& Chai, J. Y. (2006). Genotype analysis of Cryptosporidium spp. prevalent in a rural village in Hwasun-gun, Republic of Korea. The Korean journal of parasitology, 44(1), 27.

Pavlásek, I., Lávicková, M., Horák, P., Král, J., \& Král, B. (1995). Cryptosporidium varanii n. sp. (Apicomplexa: Cryptosporidiidae) in Emerald monitor (Varanus prasinus Schlegal, 1893) in captivity in Prague Zoo. Gazella, 22, 99-108.

Pavlásek, I. (1999). Cryptosporidia: biology, diagnosis, host spectrum specificity and the environment. Klinicka Mikrobiologie a Infekcni Lekarstvi, 3, 290-301.

Peacock, A. D., Chang, Y. J., Istok, J. D., Krumholz, L., Geyer, R., Kinsall, B., ... \& White, D. C. (2004). Utilization of microbial biofilms as monitors of bioremediation. Microbial Ecology, 47(3), 284-292.

Pelayo, L., Nunez, F. A., Rojas, L., Wilke, H., Furuseth Hansen, E., Mulder, B., ... \& Robertson, L. (2008). Molecular and epidemiological investigations of cryptosporidiosis in Cuban children. Annals of Tropical Medicine \& Parasitology, 102(8), 659-669.

Peng, M. M., Matos, O., Gatei, W., Das, P., Stantic-Pavlinic, M., Bern, C., ... \& Xiao, L. (2001). A comparison of Cryptosporidium subgenotypes from several geographic regions. Journal of Eukaryotic Microbiology, 48, 28s-31s.

Pennington, J., Sharpley, A., Jennings, J., Moore,, P., \& Daniel, T. (2009). Grazing Management Affects Runoff Water Quality and Forage Yield. Retrieved from https://www.uaex.edu/publications/PDF/FSA-9530.pdf

Peterson, S. B., Irie, Y., Borlee, B. R., Murakami, K., Harrison, J. J., Colvin, K. M., \& Parsek, M. R. (2011). Different methods for culturing biofilms in vitro. In Biofilm infections (pp. 251-266). Springer, New York, NY.

Pijnacker, R., Mughini-Gras, L., Vennema, H., Enserink, R., Van den Wijngaard, C. C., Kortbeek, T., \& Van Pelt, W. (2016). Characteristics of child daycare centres associated with clustering of major enteropathogens. Epidemiology \& Infection, 144(12), 25272539.

Pitlik, S. D., Fainstein, V., Garza, D., Guarda, L., Bolivar, R., Rios, A., Hopfer, R. L., \& Mansell, P. A. (1983). Human cryptosporidiosis: spectrum of disease. Report of six cases and review of the literature. Archives of Internal Medicine, 143, 2269-2275.

Platts-Mills, J. A., Babji, S., Bodhidatta, L., Gratz, J., Haque, R., Havt, A., ... \& Shakoor, S. (2015). Pathogen-specific burdens of community diarrhoea in developing countries: a multisite birth cohort study (MAL-ED). The Lancet Global health, 3(9), e564-e575. 
Plummer, J. D., Edzwald, J. K., \& Kelley, M. B. (1995). Removing Cryptosporidium by dissolved-air flotation. Journal-American Water Works Association, 87(9), 85-95.

Pohlenz, J., Moon, H. W., Cheville, N. E., \& Bemrick, W. J. (1978). Cryptosporidiosis as a probable factor in neonatal diarrhea of calves. Journal of the American Veterinary Medical Association, 172, 452-457.

Pons, W., Young, I., Truong, J., Jones-Bitton, A., McEwen, S., Pintar, K., \& Papadopoulos, A. (2015). A systematic review of waterborne disease outbreaks associated with small noncommunity drinking water systems in Canada and the United States. PLoS One, 10(10), e0141646.

Potasman, I., Paz, A., \& Odeh, M. (2002). Infectious outbreaks associated with bivalve shellfish consumption: a worldwide perspective. Clinical Infectious Diseases, 35(8), 921-928.

Pringle, J. H., \& Fletcher, M. (1983). Influence of substratum wettability on attachment of freshwater bacteria to solid surfaces. Applied and Environmental Microbiology, 45(3), 811-817.

Prystajecky, N., Huck, P. M., Schreier, H., \& Isaac-Renton, J. L. (2014). Assessment of Giardia and Cryptosporidium spp. as a microbial source tracking tool for surface water: application in a mixed-use watershed. Applied and environmental microbiology, 80(8), 2328-2336.

Putignani, L., \& Menichella, D. (2010). Global Distribution, Public Health and Clinical Impact of the Protozoan Pathogen Cryptosporidium. Interdisciplinary Perspectives On Infectious Diseases, 2010, 1-39.

PWD (2014). 2013 Annual Status Report Long Term 2 Enhanced Surface Water Treatment Rule Watershed Control Program Plan. Retreived from http://phillywatersheds.org/doc/2013\%20PWD\%20Watershed\%20Control\%20Plan\%20A nnual\%20Status\%20Report.pdf

Raccurt, C. P., Brasseur, P., Verdier, R. I., Li, X., Eyma, E., Stockman, C. P., ... \& Nevez, G. (2006). Human cryptosporidiosis and Cryptosporidium spp. in Haiti. Tropical medicine \& international health: TM \& IH, 11(6), 929-934.

RADA (2017). Home. Rada.gov.jm. Retrieved 10 January 2017, from https://rada.gov.jm

Razakandrainibe, R., Costa, D., Le Goff, L., Lemeteil, D., Ballet, J. J., Gargala, G., \& Favennec, L. (2018). Common occurrence of Cryptosporidium hominis in asymptomatic and symptomatic calves in France. PLoS neglected tropical diseases, 12(3), e0006355.

Reboredo-Fernández, A., Prado-Merini, Ó., García-Bernadal, T., Gómez-Couso, H., \& AresMazás, E. (2014). Benthic macroinvertebrate communities as aquatic bioindicators of contamination by Giardia and Cryptosporidium. Parasitology research, 113(5), 16251628. 
Rekha, K. M. H., Puttalakshmamma, G. C., \& D'Souza, P. E. (2016). Comparison of different diagnostic techniques for the detection of cryptosporidiosis in bovines. Veterinary world, 9(2), 211.

Ren, X., Zhao, J., Zhang, L., Ning, C., Jian, F., \& Wang, R. et al. (2012). Cryptosporidium tyzzeri n. sp. (Apicomplexa: Cryptosporidiidae) in domestic mice (Mus musculus). Experimental Parasitology, 130(3), 274-281.

Richardson, A. J., Frankenberg, R. A., Buck, A. C., Selkon, J. B., Colbourne, J. S., Parsons, J. W., \& Mayon-White, R. T. (1991). An outbreak of waterborne cryptosporidiosis in Swindon and Oxfordshire. Epidemiology \& Infection, 107(3), 485-495.

Robertson, L. J., Campbell, A. T., \& Smith, H. V. (1992). Survival of Cryptosporidium parvum oocysts under various environmental pressures. Applied and Environmental Microbiology, 58(11), 3494-3500.

Rochelle, P.A., Ferguson, D.M., Handojo, T.J., De Leon, R., Stewart, M.H., \& Wolfe, R.L. (1997a). An assay combining cell culture with reverse transcriptase PCR to detect and determine the infectivity of waterborne Cryptosporidium parvum. Applied and Environmental Microbiology, 63(5), 2029-2037.

Rochelle, P.A., De Leon, R., Stewart, M.H., Wolfe, R.L. (1997b). Comparison of Primers and Optimization of PCR conditions for detection of Cryptosporidium parvum and Giardia lamblia in water. Appl. Environ. Microbiol. 63, 106-114.

Rogers, J., \& Keevil, C.W. (1995). Survival of Cryptosporidium parvum oocysts in biofilm and planktonic samples in a model system. W.B. Betts et al. (Ed.). Cambridge, UK: Royal Society of Chemistry.

Rosenblatt, J. and Sloan, LM. (1993). Evaluation of an Enzyme- Linked Immunosorbent Assay for Detection of Cryptosporium spp. In Stool Specimens. Journal of Clinical Microbiology, pp 1468-1471.

Roser, D., Ashbolt, N., Ongerth, J., \& Vesey, G. (2002). Proficiency testing of Cryptosporidium and Giardia analyses-an Australian case study. Water Science and Technology: Water Supply, 2(3), 39-46.

Ruecker, N., Bounsombath, N., Wallis, P., Ong, C., Isaac-Renton, J., \& Neumann, N. (2005). Molecular Forensic Profiling of Cryptosporidium Species and Genotypes in Raw Water. Applied And Environmental Microbiology, 71(12), 8991-8994.

Ryan, K. J., \& Ray, C. G. (Eds.). (2004). Sherris medical microbiology: an introduction to infectious diseases (4th ed.) New York, NY: The McGraw Hill Companies, Inc.

Ryan, U. M., Power, M., \& Xiao, L. (2008). Cryptosporidium fayeri n. sp.(Apicomplexa: Cryptosporidiidae) from the Red Kangaroo (Macropus rufus). Journal of eukaryotic microbiology, 55(1), 22-26. 
Ryan, U. N. A., Fayer, R., \& Xiao, L. (2014). Cryptosporidium species in humans and animals: current understanding and research needs. Parasitology, 141(13), 1667-1685.

Ryan, U., Paparini, A., Monis, P., \& Hijjawi, N. (2016). It's official-Cryptosporidium is a gregarine: What are the implications for the water industry?. Water research, 105, 305313.

Ryder, D., \& Mascarenhas, E. (2007). Algal biofilms: Developing biological indicators to assess river restoration. African Journal of Aquatic Science, 32(2), 215-218.

Sambrook, J., \& Russell, D. W. (2006). The condensed protocols from Molecular cloning: $a$ laboratory manual. Cold Spring Harbor, N.Y. :Cold Spring Harbor Laboratory Press.

Santín, M., \& Trout, J.M. (2008). Livestock. In: Fayer R, Xiao L. editors. Cryptosporidium and Cryptosporidiosis. Boca Raton, FL: CRC Press. 451-483.

Sattar, S. A. (1999). Giardia cyst and Cryptosporidium oocyst survival in watersheds and factors affecting inactivation. American Water Works Association.

Sauer, K., Camper, A. K., Ehrlich, G. D., Costerton, J. W., \& Davies, D. G. (2002). Pseudomonas aeruginosa displays multiple phenotypes during development as a biofilm. Journal of bacteriology, 184(4), 1140-1154.

Savioli, L., Smith, H., \& Thompson, A. (2006). Giardia and Cryptosporidium join the 'Neglected Diseases Initiative'. Trends in Parasitology, 22, 203-208.

Schmitt, C., Schmutzler, A., \& Prinz, M. (1994). Advantages and Disadvantages of the Application of Nested PCR for Typing of Forensic Samples. In Advances in Forensic Haemogenetics (pp. 382-386). Springer, Berlin, Heidelberg.

Searcy, K. E., Packman, A. I., Atwill, E. R., \& Harter, T. (2006). Capture and retention of Cryptosporidium parvum oocysts by Pseudomonas aeruginosa biofilms. Applied and environmental microbiology, 72(9), 6242-6247.

Sendamangalam, V. (2012). The influence of biofilm structure and total interaction energy on pathogen retention by biofilm. (Master's Dissertation). Retrieved from Theses and Dissertations. 424.

Sente, C., Erume, J., Naigaga, I., Mulindwa, J., Ochwo, S., Magambo, P. K., ... \& Ocaido, M. (2016). Prevalence of pathogenic free-living amoeba and other protozoa in natural and communal piped tap water from Queen Elizabeth protected area, Uganda. Infectious diseases of poverty, 5(1), 68 .

Shahiduzzaman, M., \& Daugschies, A. (2012). Therapy and prevention of cryptosporidiosis in animals. Veterinary Parasitology, 188 (3-4), 203-214.

Shoultz, D. A., de Hostos, E. L., \& Choy, R. K. (2016). Addressing cryptosporidium infection 
among young children in low-income settings: the crucial role of new and existing drugs for reducing morbidity and mortality. PLoS neglected tropical diseases, 10(1), e0004242.

Slavin, D. (1955). Cryptosporidium meleagridis (sp. nov.). Journal of Comparative Pathology, 65, 262-266.

Smith, H. V., Patterson, W. J., Hardie, R., Greene, L. A., Benton, C., Tulloch, W., ... \& Forbes, G. I. (1989). An outbreak of waterborne cryptosporidiosis caused by post-treatment contamination. Epidemiology \& Infection, 103(3), 703-715.

Smith Warner International (2005). Recreational Carrying Capacity for Black River. Retrieved from http://www.tpdco.org/wp-content/uploads/2016/07/Black-River-location-report.pdf

Snelling, W., Xiao, L., Ortega-Pierres, G., Lowery, G., Moore, J., Rao, J. et al (2007). Cryptosporidiosis in developing countries. Journal of Infection in Developing Countries $1(3), 242-256$.

Snyder, D. B., Current, W. L., \& Russek-Cohen, E. (1988). Serologic incidence of Cryptosporidium in Delmarva broiler flocks. Poultry Science, 67, 730-735.

Spickler, A. R. (2013). Fast Fact- Cryptosporidiosis. Retrieved from http://www.cfsph.iastate.edu/DiseaseInfo/factsheets.php

Sponseller, J. K., Griffiths, J. K., \& Tzipori, S. (2014). The evolution of respiratory Cryptosporidiosis: evidence for transmission by inhalation. Clinical microbiology reviews, 27(3), 575-586.

Staggs, S. E., Keely, S. P., Ware, M. W., Schable, N., See, M. J., Gregorio, D., ... \& Villegas, E. N. (2015). The development and implementation of a method using blue mussels (Mytilus spp.) as biosentinels of Cryptosporidium spp. and Toxoplasma gondii contamination in marine aquatic environments. Parasitology research, 114(12), 46554667.

Statistical Institute of Jamaica (2017). Population by Parish. Retrieved from http://statinja.gov.jm/Demo_SocialStats/populationbyparish.aspx

Stantic-Pavlinic, M., Xiao, L., Glaberman, S., Lal, A. A., Oražen, T., Rataj-Verglez, A., ... \& Berce, I. (2003). Cryptosporidiosis associated with animal contacts. Wiener Klinische Wochenschrift, 115(3-4), 125.

Stensvold, C. R., Beser, J., Axén, C., \& Lebbad, M. (2014). High applicability of a novel method for gp60-based subtyping of Cryptosporidium meleagridis. Journal of clinical microbiology, 52(7), 2311-2319.

Stinear, T., Matusan, A., Hines, K., \& Sandery, M. (1996). Detection of a single viable Cryptosporidium parvum oocyst in environmental water concentrates by reverse transcription-PCR. Applied and Environmental Microbiology, 62(9), 3385-3390. 
Stoodley, P., Sauer, K., Davies, D., \& Costerton, J. (2002). Biofilms as complex differentiated communities. Annual Review of Microbiology, 56, 187-209.

Strong, W. B., Gut, J., \& Nelson, R. G. (2000). Cloning and sequence analysis of a highly polymorphic Cryptosporidium parvum gene encoding a 60-kilodalton glycoprotein and characterization of its 15-and 45-kilodalton zoite surface antigen products. Infection and immunity, 68(7), 4117-4134.

Sturbaum, G. D., Reed, C., Hoover, P. J., Jost, B. H., Marshall, M. M., \& Sterling, C. R. (2001). Species-specific, nested PCR-restriction fragment length polymorphism detection of single Cryptosporidium parvum oocysts. Applied and environmental microbiology, 67(6), 2665-2668.

Sturbaum, G., Klonicki, P., Marshall, M., Jost, B., Clay, B., \& Sterling, C. (2002). Immunomagnetic Separation (IMS)-Fluorescent Antibody Detection and IMS-PCR Detection of Seeded Cryptosporidium parvum Oocysts in Natural Waters and Their Limitations. Applied And Environmental Microbiology, 68(6), 2991-2996.

Sulaiman, I. (1998). Differentiating Human from Animal Isolates of Cryptosporidium parvum. Emerging Infectious Diseases, 4(4), 681-685.

Sulaiman, I. M., Xiao, L., \& Lal, A.A. (1999). Evaluation of Cryptosporidium parvum genotyping techniques. Appl. Environ. Microbiol. 65, 4431-4435.

Swai, E. S., \& Schoonman, L. (2010). Investigation into the prevalence of Cryptosporidium infection in calves among small-holder dairy and traditional herds in Tanzania. Veterinary medicine international, 2010.

Tahvildar-Biderouni, F., \& Salehi, N. (2014). Detection of Cryptosporidium infection by modified ziehl-neelsen and PCR methods in children with diarrheal samples in pediatric hospitals in Tehran. Gastroenterology and hepatology from bed to bench, 7(2), 125.

Takhistov, P., \& George, B. (2004). Early events and pattern formation in Listeria monocytogenes biofilms. Biofilms, 1(4), 351-359.

Tenter, A., Barta, J., Beveridge, I., Duszynski, D., Mehlhorn, H., \& Morrison, D. et al. (2002). The conceptual basis for a new classification of the coccidia. International Journal For Parasitology, 32(5), 595-616.

Thermo Scientific (2009). T042-TECHNICAL BULLETIN NanoDrop Spectrophotometers 260/280 and 260/230 Ratios. Retrieced from file:///Users/amandamorris/Desktop/nanodrop.pdf

Tonetto, A. F., Peres, C. K., \& Branco, C. C. Z. (2012). A new method to quantify macroalgae and a practical sampler for experimentation in lotic habitats. Brazilian Journal of Biology, 72(4), 853-857.

Tumwine, J. K., Kekitiinwa, A., Nabukeera, N., Akiyoshi, D. E., Rich, S. M., Widmer, G., ... \& 
Tzipori, S. (2003). Cryptosporidium parvum in children with diarrhea in Mulago Hospital, Kampala, Uganda. The American journal of tropical medicine and hygiene, 68(6), 710-715.

Tyzzer, E. (1907). A sporozoan found in the peptic glands of the common mouse. Experimental Biology and Medicine, 5(1), 12-13.

Tyzzer, E. (1910). An extracellular coccidium, Cryptosporidium muris (gen. et sp. Nov.), of the gastric glands of the common mouse. Indian Journal of Medical Research, 23(3), 487509.

Tyzzer, E. (1912). Cryptosporidium parvum (sp. nov.), a coccidium found in the small intestine of the common mouse. Archiv Protistenkunde, 26, 394-412.

Tzipori, S., Campbell, I., Sherwood, D., Snodgrass, D. R., \& Whitelaw, A. (1980). An outbreak of calf diarrhoea attributed to cryptosporidial infection. Veterinary Record, 107, 579-580.

Tzipori, S., \& Widmer, G. (2008). A hundred-year retrospective on cryptosporidiosis. Trends In Parasitology, 24(4), 184-189.

US EPA (2005). Method 1623: Cryptosporidium and Giardia in water by filtration/IMS/FA. Retrieved from https://www.epa.gov/sites/production/files/2015-07/documents/epa1623.pdf

US EPA (2001). Cryptosporidium: Drinking Water Health Advisory. Retrieved from https://www.epa.gov/sites/production/files/2015-10/documents/cryptosporidiumreport.pdf

US EPA (2012). Method 1623.1 Microscopy Visual Guide [Photograph]. Retrieved from http://www.cofes.org.ar/descargas/relas/8_jornada/12_EPA\%201623\%202012.pdf

USDA (2017). USDA's National Agricultural Statistics Service Wisconsin Field Office. Retrieved from https://www.nass.usda.gov/Statistics_by_State/Wisconsin/Publications/Dairy/

Vetterling, J., Jervis, H., Merril, T., \& Sprinz, H. (1971). Cryptosporidium wrairi sp. n. from the guinea pig Cavia porcellus, with an emendation of the genus. Journal of Protozoology, $' 18,243-247$.

Vernier Software \& Technology (n.d.). Total Water Hardness. Retrieved from http://www2.vernier.com/sample_labs/WQV-14-COMP-total_water_hardness.pdf

Vohra, P., Sharma, M., \& Chaudary, U. (2012). A comprehensive review of diagnostic techniques for detection of Cryptosporidium parvum in stool samples. J Pharm, 2(5), 1526.

Vorregaard (2008). Comstat2 - a modern 3D image analysis environment for biofilms. Retrieved from http://www2.imm.dtu.dk/pubdb/views/edoc_download.php/5628/pdf/imm5628.pdf 
VWR (2017). Envirochek ${ }^{\mathrm{TM}}$ and Envirochek ${ }^{\mathrm{TM}} H V$ Sampling Capsules, Pall Laboratory | VWR. Us.vwr.com. Retrieved 10 January 2017, from https://us.vwr.com/store/product/4830054/envirochek-and-envirochek-hv-samplingcapsules-pall-laboratory

Waldman, E., Tzipori, S., \& Forsyth, J. R. (1986). Separation of Cryptosporidium species oocysts from feces by using a percoll discontinuous density gradient. Journal of clinical microbiology, 23(1), 199-200.

Waldron, L. S., Ferrari, B. C., \& Power, M. L. (2009). Glycoprotein 60 diversity in C. hominis and C. parvum causing human cryptosporidiosis in NSW, Australia. Experimental parasitology, 122(2), 124-127.

Walker, M., Montemagno, C., \& Jenkins, M. (1998). Source water assessment and nonpoint sources of acutely toxic contaminants: a review of research related to survival and transport of Cryptosporidium parvum. Water Resources Research, 34, 3383-3392.

Wang, R., Zhang, L., Axén, C., Bjorkman, C., Jian, F., Amer, S., ... \& Zhao, Z. (2014). Cryptosporidium parvum IId family: clonal population and dispersal from Western Asia to other geographical regions. Scientific reports, $4,4208$.

Ward, P. I., Deplazes, P., Regli, W., Rinder, H., \& Mathis, A. (2002). Detection of eight Cryptosporidium genotypes in surface and waste waters in Europe. Parasitology, 124, 359-368.

Warnecke, M. (2006). Cryptosporidium oocyst interactions with drinking water pipe biofilms. Coop. Res. Cent. Water Qual. Treat. Salisb. S. Aust. Aust.

Water Research Foundation. (2016). Milwaukee Water Works \& Ozone. Retrieved from http://www.waterrf.org/the-foundation/Documents/Milwaukee-Water-Works-andOzone.pdf

Waterborne Inc. (2010). Catalog Nr: A400FLK Crypt-a-Glo ${ }^{\mathrm{TM}} \mathrm{G} / \mathrm{C}$ Direct Comprehensive Kit. Retrieved from https://static1.squarespace.com/static/55751402e4b017c5045aa9a0/t/5720246b86db4347 6f5e934a/1461724267875/A400FLK_prod_insert-1.pdf

Westerling, K. (2014). Do-It-Yourself Crypto Detection. Retrieved from https://www.wateronline.com/doc/do-it-yourself-crypto-detection-0001

WHO. (2009). Risk Assessment of Cryptosporidium in Drinking Water. World Health Organization. Retrieved from http://apps.who.int/iris/bitstream/handle/10665/70117/WHO_HSE_WSH_09.04_eng.pdf; jsessionid $=93064727 \mathrm{BA} 281 \mathrm{FFC5D742F5EF7EAE1FB}$ ?sequence=1

Widmer, G. (1998). Genetic heterogeneity and PCR detection of Cryptosporidium parvum. Advances in Parasitology, 40, 223-239. 
Wikimedia Commons (2012). File: Jamaica, administrative divisions (parishes) - en monochrome.svg Jamaica, [Photograph]. Retrieved from

https://commons.wikimedia.org/wiki/File:Jamaica,_administrative_divisions_(parishes)__en_-_monochrome.svg

Wolyniak DiCesare, E. A., Hargreaves, B. R., \& Jellison, K. L. (2009). Retention and release of Cryptosporidium parvum oocysts by experimental biofilms composed of a natural stream microbial community. Applied and environmental microbiology, 75(13), 4624-4626.

Wolyniak, E.A., Hargreaves, B.R., \& Jellison, K.L. (2010). Seasonal retention and release of Cryptosporidium parvum oocysts by environmental biofilms in the laboratory. Applied and environmental microbiology, 76(4), 1021-1027.

Wolyniak DiCesare, E., Hargreaves, B., \& Jellison, K. (2012a). Biofilms Reduce Solar Disinfection of Cryptosporidium parvum Oocysts. Applied And Environmental Microbiology, 78(12), 4522-4525.

Wolyniak DiCesare, E., Hargreaves, B., \& Jellison, K. (2012b). Biofilm Roughness Determines Cryptosporidium parvum Retention in Environmental Biofilms. Applied And Environmental Microbiology, 78(12), 4187-4193.

World Water \& Environmental Resources Congress (2004). World Water Congress 2003: World Water \& Environmental Resources Congress 2003 and related symposia. Reston, $\mathrm{Va}$ : American Society of Civil Engineers.

Xagoraraki, I., \& Harrington, G. W. (2004). Zeta potential, dissolved organic carbon, and removal of Cryptosporidium oocysts by coagulation and sedimentation. Journal of environmental engineering, 130(12), 1424-1432.

Xiao, L., Escalante, L., Yang, C., Sulaiman, I., Escalante, A. A., Montali, R. J., ... \& Lal, A. A. (1999). Phylogenetic analysis of Cryptosporidiumparasites based on the small-subunit rRNA gene locus. Applied and environmental microbiology, 65(4), 1578-1583.

Xiao, L., Alderisio, K., Limor, J., Royer, M., \& Lal, A. (2000). Identification of Species and Sources of Cryptosporidium Oocysts in Storm Waters with a Small-Subunit rRNA-Based Diagnostic and Genotyping Tool. Applied And Environmental Microbiology, 66(12), 5492-5498.

Xiao, L., Singh, A., Limor, J., Graczyk, T. K., Gradus, S., \& Lal, A. (2001). Molecular characterization ofCryptosporidium oocysts in samples of raw surface water and wastewater. Applied and environmental microbiology, 67(3), 1097-1101.

Xiao, L., Alderisio, K. A., \& Jiang, J. (2006). Detection of Cryptosporidium oocysts in water: effect of the number of samples and analytic replicates on test results. Applied and environmental microbiology, 72(9), 5942-5947.

Xiao, L., Fayer, R., Ryan, U., \& Upton, S. (2004). Cryptosporidium Taxonomy: Recent 
Advances and Implications for Public Health. Clinical Microbiology Reviews, 17(1), 7297.

Xiao, L., \& Feng, Y. (2017). Molecular epidemiologic tools for waterborne pathogens Cryptosporidium spp. and Giardia duodenalis. Food and Waterborne Parasitology.

Xiao, L., \& Ryan, U. M. (2008). Molecular Epidemiology. In: Fayer R, Xiao L, editors. Cryptosporidium and Cryptosporidiosis. 2nd ed. Boca Raton, FL: CRC Press and IWA Publishing. 119-17.

Zhou, L., Singh, A., Jiang, J., Xiao, L. (2003) Molecular surveillance of Cryptosporidium spp. in raw wastewater in Milwaukee: implications for understanding outbreak occurrence and transmission dynamics, Journal of Clinical Microbioly, 41(11), 5254-5257

Yang, W., Chen, P., Villegas, E., Landy, R., Kanetsky, C., \& Cama, V. et al. (2008). Cryptosporidium Source Tracking in the Potomac River Watershed. Applied And Environmental Microbiology, 74(21), 6495-6504.

Ziegler, P. E., Wade, S. E., Schaaf, S. L., Stern, D. A., Nadareski, C. A., \& Mohammed, H. O. (2007). Prevalence of Cryptosporidium species in wildlife populations within a watershed landscape in southeastern New York State. Veterinary parasitology, 147(1-2), 176-184. 